Método híbrido de alta ordem para escoamentos compressíveis

Vitor Alves Pires 

SERVIÇO DE PÓS-GRADUAÇÃO DO ICMC-USP

Data de Depósito:

Assinatura:

\title{
Método híbrido de alta ordem para escoamentos compressíveis
}

\author{
Vitor Alves Pires
}

Orientadora: Profa. Dra. Maria Luísa Bambozzi de Oliveira

Dissertação apresentada ao Instituto de Ciências Matemáticas e de Computação - ICMC-USP, como parte dos requisitos para obtenção do título de Mestre em Ciências - Ciências de Computação e Matemática Computacional. EXEMPLAR DE DEFESA

\author{
USP - São Carlos
}

Abril de 2015 
Ficha catalográfica elaborada pela Biblioteca Prof. Achille Bassi e Seção Técnica de Informática, ICMC/USP, com os dados fornecidos pelo(a) autor(a)

\begin{tabular}{|c|c|}
\hline P $667 \mathrm{~m}$ & $\begin{array}{l}\text { Pires, Vitor Alves } \\
\text { Método híbrido de alta ordem para escoamentos } \\
\text { compressiveis / Vitor Alves Pires; orientadora Maria } \\
\text { Luísa Bambozi Oliveira. -- São Carlos, } 2015 . \\
\quad 95 \text { p. }\end{array}$ \\
\hline & $\begin{array}{l}\text { Dissertação (Mestrado - Programa de Pós-Graduação } \\
\text { em Ciências de Computação e Matemática } \\
\text { Computacional) -- Instituto de Ciências Matemáticas } \\
\text { e de Computação, Universidade de São Paulo, } 2015 . \\
\text { 1. Métodos numéricos. 2. Análise numérica. 3. } \\
\text { Equaçoses Diferencias Parciais. I. Oliveira, Maria } \\
\text { Luísa Bambozi, orient. II. Título. }\end{array}$ \\
\hline
\end{tabular}




\section{Agradecimentos}

Agradeço primeiramente a Deus, que sempre guia meus passos com muita sabedoria, carinho e atenção. Agradeço também aos meus pais que sempre me encorajam e me motivam nos momentos mais importantes da minha vida, principalmente em todos esses anos de estudos. Além disso, gostaria de agradecer também a minha namorada pela compreensão e companheirismo durante todo o caminho. E de uma forma especial a todos os meus parentes, amigos e professores que contribuíram para o meu desenvolvimento pessoal e profissional. Agradeço também a CAPES pelo apoio financeiro para a realização do projeto de pesquisa. 
presença de onda de choque e vórtices de pequena escala exigem métodos numéricos mais sofisticados para simular escoamentos compressíveis em velocidades altas. Alguns desses métodos produzem resultados adequados para regiões com função suave, embora os mesmos não possam ser utilizados diretamente em regiões com função descontínua, resultando em oscilações espúrias. Dessa forma, métodos foram desenvolvidos para solucionar esse problema, apresentando um bom desempenho para regiões com função descontínua; entretanto, estes possuem termos de alta dissipação.

Para evitar os problemas encontrados, foram desenvolvidos os métodos híbridos, onde dois métodos com características ideais para cada região são combinados através de uma função detectora que analisa numericamente a variação de uma quantidade em uma região através de fórmulas que envolvem derivadas.

Um detector de descontinuidades foi desenvolvido a partir da revisão bibliográfica de diversos métodos numéricos híbridos existentes, sendo avaliadas as principais desvantagens e limitações de cada um.

Diversas comparações entre o novo detector e os detectores de descontinuidades já desenvolvidos foram realizadas através da aplicação em funções unidimensionais e bidimensionais.

Finalmente, o método híbrido foi aplicado para a solução das equações de Euler unidimensionais e bidimensionais.

Palavras-chave: métodos híbridos, detector de descontinuidades, equações de Euler. 

7 he presence of shock and small-scale vortices require more sophisticated numerical methods to simulate compressible flows at high speeds. Some of these methods produce good results for regions with smooth function, altough they cannot be used directly in regions with discontinuous functions, resulting in spurious oscillations. Thus, methods have been developed to solve this problem, showing a good performance for regions with discontinuous functions; however, these methods contain high dissipation terms.

To avoid the problems encountered, hybrid methods have been developed, where two methods with ideal characteristics for each region are combined through a function that analyze numerically the variation of a quantity in the region using formulas involving derivatives.

A discontinuity detector was developed from the literature review of several existing hybrid methods, evaluating the main disadvantages and limitations of each.

The new detector and other developed discontinuity detectors were compared by applying on one and two-dimensional functions.

Finally, the hybrid method was applied fo the solution of one and twodimensional Euler equations.

Keywords: hybrid methods, descontinuity detector, Euler equations. 



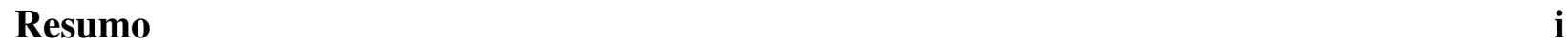

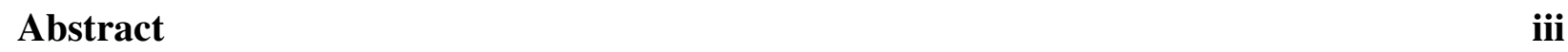

1 Introdução $\quad 1$

2 Detector de Descontinuidades $\quad 3$

2.1 Diferenças Centrais . . . . . . . . . . . . . . . . . . . . 4

2.1.1 Análise de Fourier . . . . . . . . . . . . . . . . . 6

2.2 Caso Unidimensional . . . . . . . . . . . . . . . . . . . . . . . 7

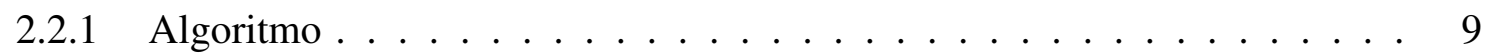

2.3 Caso Bidimensional . . . . . . . . . . . . . . . . . . . . . . . . . . 9

2.4 Observações . . . . . . . . . . . . . . . . . . . 10

3 O Valor Limiar $\quad 11$

3.1 Construção de Polinômios . . . . . . . . . . . . . . . . . . . . . . . . . . . . . .

3.1 .1 Polinômio para Função Suave . . . . . . . . . . . . . . . . . . . . . . . 12

3.1 .2 Polinômio para Função Não Suave . . . . . . . . . . . . . . . . . . . . . . . . . . 12

3.2 Análise . . . . . . . . . . . . . . . . . . . 13

3.2 .1 Função Não suave . . . . . . . . . . . . . . . . . . . . . . . . . . . . . . . . . . 14

3.2 .2 Soma de Derivadas . . . . . . . . . . . . . . . . . . . . . . . . . . . . . . . . . . . 14

3.2 .3 Razão do Detector Desenvolvido . . . . . . . . . . . . . . . . . . 15

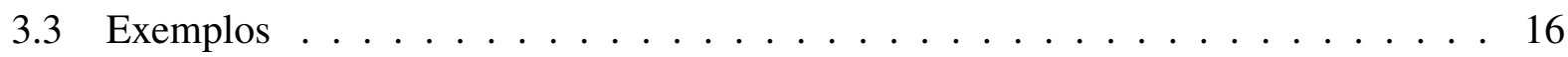

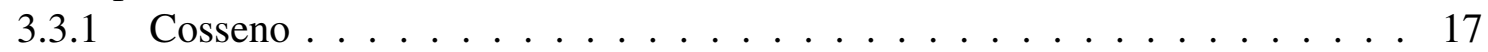

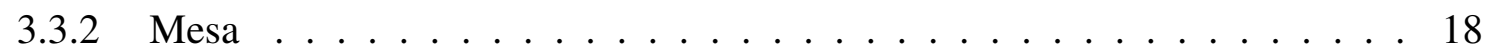

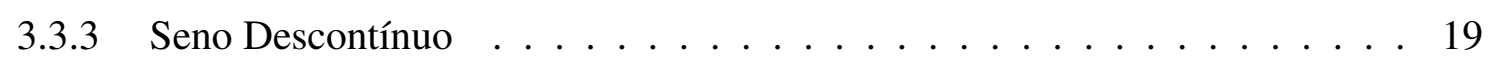

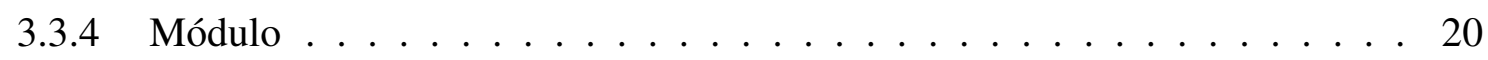

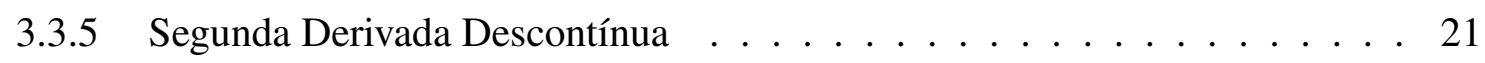

3.3.6 Terceira Derivada Descontínua . . . . . . . . . . . . . . . . 21

3.3 .7 Frequência Alta . . . . . . . . . . . . . . . 23

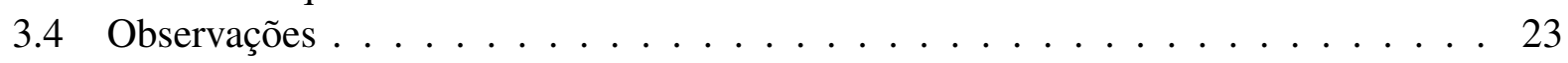

4 Resultados $\quad 25$

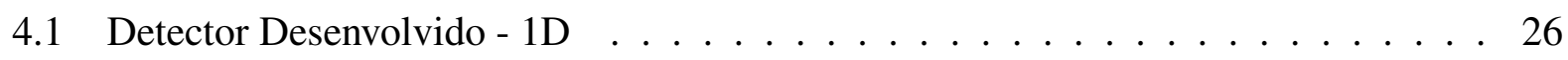

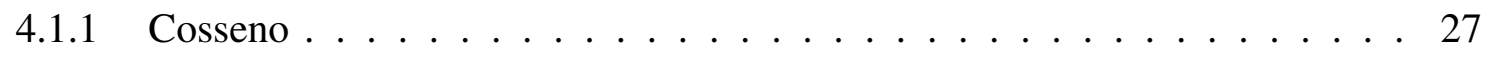




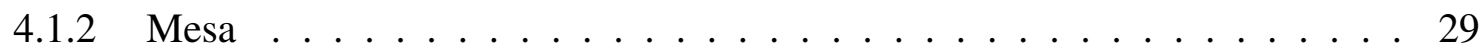

4.1 .3 Seno Descontínuo . . . . . . . . . . . . . . . . 32

4.1 .4 Módulo . . . . . . . . . . . . . . . . . . 33

4.1 .5 Frequência Alta . . . . . . . . . . . . . . . . . . 36

4.1.6 Aplicação do Método Híbrido - 1D . . . . . . . . . . . . . . . 37

4.2 Equações de Euler - 1D . . . . . . . . . . . . . . . . . . . . . . . . 37

4.3 Detector Desenvolvido - 2D . . . . . . . . . . . . . . . 42

4.3 .1 Suave .............................. 45

4.3.2 Descontínua-Circunferência . . . . . . . . . . . 45

4.3 .3 Descontínua - Triângulo . . . . . . . . . . . . . . . . . 50

4.3 .4 Aplicação do Método Híbrido - 2D . . . . . . . . . . . . . . 50

4.4 Equações de Euler $-2 \mathrm{D} \ldots \ldots \ldots \ldots \ldots \ldots$

4.4 Observações . . . . . . . . . . . . . . . . . . 59

5 Conclusão $\quad 67$

$\begin{array}{lrr}\text { A Método WENO } & 69\end{array}$

A.1 Análise de Fourier . . . . . . . . . . . . . . . . . . . 72

B Equações de Euler $\quad \mathbf{7 5}$

B.1 Caso Unidimensional . . . . . . . . . . . . . . . . . . . . . . . 75

B.1.1 Forma Vetorial . . . . . . . . . . . . . . 75

B.1.2 Equação de Estado . . . . . . . . . . . . . . . . . . . . 76

B.1.3 Separação de Fluxo . . . . . . . . . . . . . . . . . . . . . . . . . . . . . . . 77

B.1.4 Algoritmo . . . . . . . . . . . . . . . . . 79

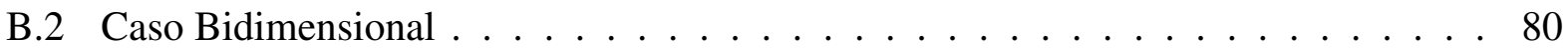

B.2.1 Forma Vetorial . . . . . . . . . . . . . . . . . 80

B.2.2 Equação de Estado . . . . . . . . . . . . . . . . . . 80

B.2.3 Separação de Fluxo . . . . . . . . . . . . . . . . . . . . . 81

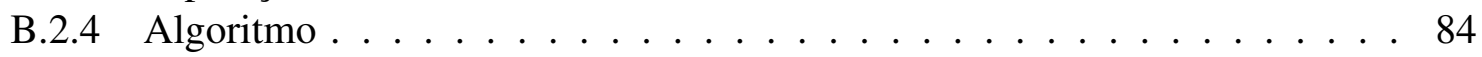

C Detectores de Descontinuidades $\quad 85$

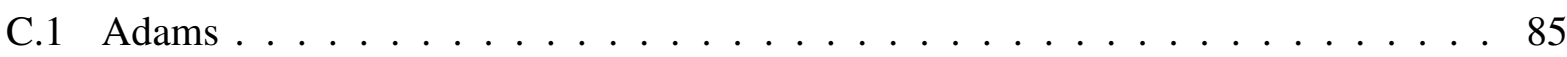

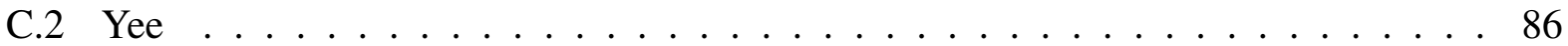

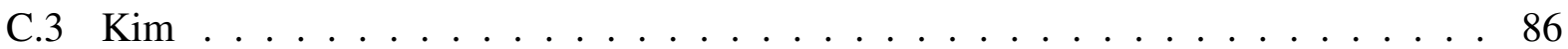

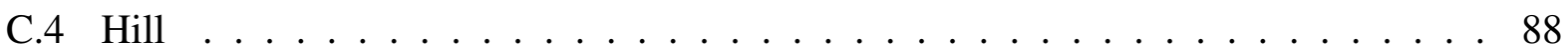

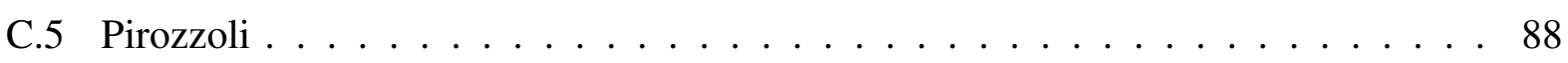

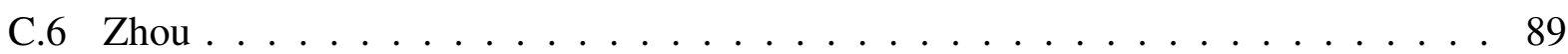




\section{Lista de Figuras}

2.1 Análise de Fourier - Diferenças Centrais . . . . . . . . . . . . . . . . 7

3.1 Relação $2^{n}$ - Função Cosseno . . . . . . . . . . . . . . . . . . . . . . . . . 17

3.2 Relação $2^{n}$ - Função Mesa . . . . . . . . . . . . . . . . . . . . . . 18

3.3 Relação $2^{n}$ - Seno Descontínuo . . . . . . . . . . . . . . . . . . . . . . . . . 19

3.4 Relação $2^{n}$ - Função Módulo . . . . . . . . . . . . . . . . . . . . . . 20

3.5 Relação $2^{n}$ - Segunda Derivada Descontínua . . . . . . . . . . . . . . . . . 21

3.6 Relação $2^{n}$ - Terceira Derivada Descontínua . . . . . . . . . . . . . . . . . . . 22

3.7 Relação $2^{n}$ - Alta Frequência . . . . . . . . . . . . . . . . . 23

4.1 Detectores 1D - Função Cosseno . . . . . . . . . . . . . . . . . . . . 27

4.2 Detectores 1D - Função Cosseno - continuação . . . . . . . . . . . . . . . . 28

4.3 Detectores 1D - Função Mesa . . . . . . . . . . . . . . . . . . . . . . . . . 29

4.4 Detectores 1D - Função Mesa - continuação . . . . . . . . . . . . . . . . 30

4.5 Detectores 1D - Função Seno Descontínuo . . . . . . . . . . . . . . . . . . . 31

4.6 Detectores 1D - Função Seno Descontínuo - continuação . . . . . . . . . . . . . 32

4.7 Detectores 1D - Função Módulo . . . . . . . . . . . . . . . . . . . . 33

4.8 Detectores 1D - Função Módulo - continuação . . . . . . . . . . . . . . . . 34

4.9 Detectores 1D - Função Frequência Alta . . . . . . . . . . . . . . . . . 35

4.10 Detectores 1D - Função Frequência Alta - continuação . . . . . . . . . . . . . . 36

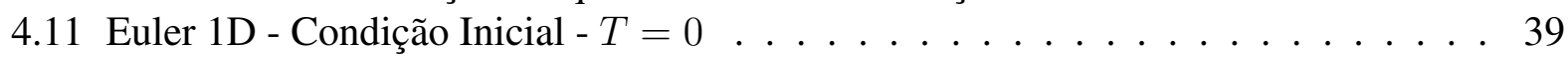

4.12 Euler 1D - Híbrido $-N_{x}=201, C F L=0.2$ e $T=1.8 \ldots \ldots \ldots \ldots$

4.13 Euler 1D - Híbrido $-N_{x}=801, C F L=0.2$ e $T=1.8 \ldots \ldots \ldots \ldots$. . . . 41

4.14 Euler 1D - WENO e Híbrido - $C F L=0.2$ e $T=1.8 \ldots \ldots \ldots \ldots$. . . . . 42

4.15 Comparação 1D - WENO e Híbrido - $N_{x}=201, C F L=0.2$ e $T=1.8 \ldots$. . . . 43

4.16 Comparação 1D - WENO e Híbrido - $N_{x}=801, C F L=0.2$ e $T=1.8 \ldots$. . . . 44

4.17 Novo detector 2D - Suave - $N_{x}=101$ e $N_{y}=101 \ldots \ldots \ldots \ldots$. . . . . . 46

4.18 Novo detector 2D - Circunferência - $N_{x}=101$ e $N_{y}=101 \ldots$. . . . . . . . . 47

4.19 Novo detector 2D - Circunferência - $N_{x}=401$ e $N_{y}=401 \ldots \ldots$. . . . . . 48

4.20 Novo detector 2D - Triângulo - $N_{x}=101$ e $N_{y}=101 \ldots \ldots$. . . . . . . . . . 49

4.21 Novo detector 2D - Triângulo $-N_{x}=401$ e $N_{y}=401 \ldots \ldots \ldots$. . . . . . 51

4.22 Euler 2D - Condição Inicial Circunferência - T=0 . . . . . . . . . . 53

4.23 Euler 2D - WENO - $N_{x}=N_{y}=101, C F L=0.05$ e $T=0.5$ - Circunferência $\quad . \quad 54$

4.24 Euler 2D - WENO - Perfil em $y=0-N_{x}=N_{y}=101, C F L=0.05$ e $T=0.5$ -

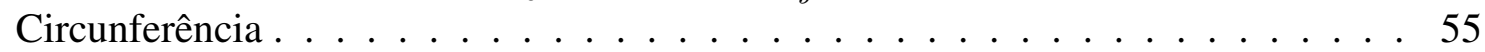


4.25 Euler 2D - Híbrido - $N_{x}=N_{y}=101, C F L=0.05$ e $T=0.5$ - Circunferência . . 56

4.26 Euler 2D - Híbrido - Perfil em $y=0-N_{x}=N_{y}=101, C F L=0.05$ e $T=0.5$ Circunferência . . . . . . . . . . . . . . . 57

4.27 Euler 2D - Comparação - Perfil em $y=0-N_{x}=N_{y}=101, C F L=0.05$ e

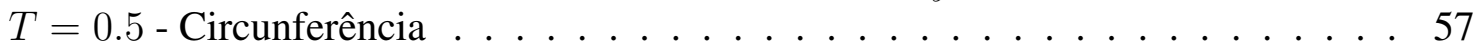

4.28 Euler 2D - WENO - Perfil em $y=0-N_{x}=N_{y}=401, C F L=0.05$ e $T=0.5-$

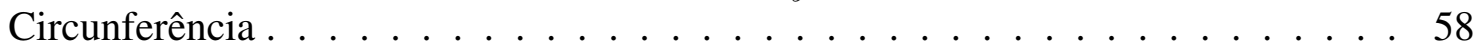

4.29 Euler 2D - Híbrido - Perfil em $y=0-N_{x}=N_{y}=401, C F L=0.05$ e $T=0.5$ -

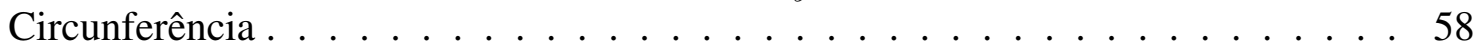

4.30 Euler 2D - Comparação - Perfil em $y=0-N_{x}=N_{y}=401, C F L=0.05 \mathrm{e}$ $T=0.5$ - Circunferência . . . . . . . . . . . . . . 59

4.31 Euler 2D - WENO - $N_{x}=83$ e $N_{y}=31, C F L=0.3$ e $T=5.0$ - Choque Incidente 60

4.32 Euler 2D - WENO - Perfil em $y=0.75-N_{x}=83$ e $N_{y}=31, C F L=0.3 \mathrm{e}$ $T=5.0$ - Choque Incidente . . . . . . . . . . . . . . . . 61

4.33 Euler 2D - Híbrido - $N_{x}=83$ e $N_{y}=31, C F L=0.3$ e $T=5.0$ - Choque Incidente 61

4.34 Euler 2D - Híbrido - Perfil em $y=0.75-N_{x}=83$ e $N_{y}=31, C F L=0.3 \mathrm{e}$ $T=5.0$ - Choque Incidente . . . . . . . . . . . . . . . 62

4.35 Euler 2D - Comparação - Perfil em $y=0.75-N_{x}=83$ e $N_{y}=31, C F L=0.3$ e $T=5.0$ - Choque Incidente . . . . . . . . . . . . . . . . 62

4.36 Euler 2D - WENO - $N_{x}=165$ e $N_{y}=61, C F L=0.3$ e $T=5.0$ - Choque Incidente 63

4.37 Euler 2D - WENO - Perfil em $y=0.75-N_{x}=165$ e $N_{y}=61, C F L=0.3 \mathrm{e}$ $T=5.0$ - Choque Incidente . . . . . . . . . . . . . . 63

4.38 Euler 2D - Híbrido - $N_{x}=165$ e $N_{y}=61, C F L=0.3$ e $T=5.0$ - Choque Incidente . . . . . . . . . . . . . . . . . . 64

4.39 Euler 2D - Híbrido - Perfil em $y=0-N_{x}=165$ e $N_{y}=61, C F L=0.3 \mathrm{e}$ $T=5.0$ - Choque Incidente . . . . . . . . . . . . . . . . . 64

4.40 Euler 2D - Comparação - Perfil em $y=0-N_{x}=165$ e $N_{y}=61, C F L=0.3 \mathrm{e}$ $T=5.0$ - Choque Incidente . . . . . . . . . . . . . . . 65

A.1 Análise de Fourier - WENO . . . . . . . . . . . . . . . . . . . . . 74 


\section{Lista de Tabelas}

4.1 Tabela de erros - Derivada da função Cosseno - 1D . . . . . . . . . . . . . . . . . 37

4.2 Tabela de tempos de execução - Euler 1D . . . . . . . . . . . . . . . 41

4.3 Tabela de erros - Derivada da função Suave - Direção $x$ - 2D . . . . . . . . . . . 50

4.4 Tabela de erros - Derivada da função Suave - Direção $y$ - 2D . . . . . . . . . . . . 50

4.5 Tabela de tempos de execução - Euler 2D - Circunferência . . . . . . . . . . . . . . 55

4.6 Tabela de tempos de execução - Euler 2D - Choque Incidente . . . . . . . . . . . . 59 


\section{CAPÍTULO \\ 1 \\ Introdução}

A solução numérica de escoamentos compressíveis em velocidades altas exige um custo computacional alto com a utilização de malhas bastante refinadas e passos de tempo pequenos. A presença de ondas de choque e vórtices de pequena escala exigem métodos numéricos mais sofisticados para simular o escoamento e calcular as derivadas espaciais discretas $[13,16,26]$.

Alguns desses métodos, como o método Compacto [16], produzem resultados adequados para regiões de funções com pouca variação em sua quantidade, denominadas regiões com função suave, embora os mesmos não possam ser utilizados diretamente em regiões que apresentam funções com descontinuidades, produzindo oscilações espúrias. Para solucionar esse problema, outros métodos, como ENO [26] e WENO [13], foram desenvolvidos, apresentando um bom desempenho para regiões com função descontínua. Entretanto, estes possuem termos de alta dissipação, podendo apresentar perda de qualidade quando aplicados em regiões com função suave [21].

Para evitar as dificuldades encontradas, diversos métodos de ordem alta foram desenvolvidos ou adaptados $[2,6,11,12,14,23,33]$. Porém, estes ainda possuem algumas limitações que não permitem sua aplicação de uma forma geral, já que é necessário definir um valor limite para a separação das regiões com função suave ou descontínua.

Pode-se categorizar alguns desses métodos como híbridos, onde dois métodos com características ideais para cada região (com função descontínua ou suave) são combinados através de uma função detectora. Esta função, em geral, analisa numericamente a variação de uma quantidade em uma região através de fórmulas que envolvem derivadas.

Para o sucesso na construção de métodos híbridos, deve-se considerar com cuidado tanto os métodos que são utilizados para cada região quanto a formulação da função detectora. Métodos básicos já desenvolvidos, como de diferenças centrais, Compacto [16], ENO [26] ou WENO [13] 
podem ser aplicados, mas com uma função detectora que seja capaz de se adaptar a cada tipo de problema. Sendo assim, com a revisão bibliográfica e a avaliação das principais desvantagens e limitações de cada função detectora foi possível desenvolver um novo detector de descontinuidades que avaliasse corretamente cada tipo de região.

Para a solução numérica de escoamentos compressíveis em velocidades altas com maior qualidade e sem aumentar o custo computacional é necessário que o método numérico híbrido desenvolvido de ordem alta seja capaz de amortecer somente oscilações espúrias e que as ondas de choque sejam tratadas precisamente, adaptando-se ao problema considerado.

Dessa forma, o principal objetivo é aplicar esse método em escoamentos no regime laminar unidimensionais e bidimensionais para resolver as equações de Euler e verificar os resultados obtidos com o mesmo.

Essa dissertação é organizada da seguinte forma. No Capítulo 2 é apresentado o novo detector de descontinuidades desenvolvido. Já o Capítulo 3 apresenta uma análise de valores limite para a definição do detector. No Capítulo 4 são apresentados os resultados para o novo detector desenvolvido e a solução das equações de Euler unidimesnionais e bidimensionais para diferentes condições iniciais. Finalmente, no Capítulo 5, são apresentadas as conclusões e as contribuições para trabalhos futuros. 


\section{Detector de Descontinuidades}

Neste capítulo é apresentado o desenvolvimento de um novo detector de descontinuidades, baseado na análise de detectores existentes. Além disso, é considerado o caso unidimensional e sua extensão para o caso bidimensional, que permitem avaliar o desempenho do detector para diferentes funções.

Com a solução numérica de escoamentos compressíveis em velocidades altas, tem-se um alto custo computacional com a utilização de malhas bastante refinadas e passos de tempo pequenos. Dessa forma, pela possível presença de ondas de choque e vórtices de pequena escala são necessários métodos numéricos mais sofisticados de alta ordem para calcular as derivadas espaciais discretas e simular o escoamento.

Nota-se que, com o desenvolvimento de métodos numéricos de alta ordem, alguns [16] funcionam corretamente para regiões com função suave, considerando regiões de funções com pouca variação em sua quantidade, mas geram oscilações espúrias em regiões com função descontínua. Já métodos [13, 26] que funcionam corretamente para regiões descontínuas apresentam perda de qualidade quando aplicados em regiões com função suave.

Assim, com a finalidade de solucionar esses problemas, uma ideia natural é combinar dois métodos com características ideais para cada tipo de região (com função suave ou descontínua). Para as regiões com função suave pode-se aplicar métodos como o Compacto [16] e o de diferenças centrais; já para regiões que apresentam descontinuidades, métodos como ENO [26] e WENO [13].

Dessa maneira, constrói-se um método híbrido com a combinação linear

$$
\text { Híbrido }=\sigma M S+(1-\sigma) M D
$$


em que $M S$ representa um método ideal para região com função suave e $M D$ para a região com função descontínua.

Observa-se que $\sigma$ é um parâmetro que vale 0 ou 1, sendo que, pela equação (2.1), se $\sigma=1$ aplica-se apenas o método para a região com função suave, enquanto que, para $\sigma=0$, aplica-se apenas o método que funciona corretamente para regiões com função descontínua.

Sendo assim, é necessário considerar uma função detectora $(\sigma)$ para definir quais regiões podem ser consideradas com função suave e quais com função descontínua, possibilitando decidir qual método deve ser utilizado para uma determinada região.

Com a revisão bibliográfica e o estudo de diversos métodos numéricos híbridos existentes, foi possível avaliar as principais vantagens e desvantagens de cada função detectora, sendo estas testadas e avaliadas para diferentes funções.

Através dos métodos estudados, observou-se que os desenvolvidos por Adams [2] e Pirozzoli [21] apresentam um ideia muito simples para a construção da função detectora, avaliando apenas a variação da função. Já os métodos desenvolvidos por Zhou [36], Yee [33] e Kim [14] possuem ideias similares entre si com pequenas variações na forma de avaliar a existência da região descontínua, sendo que a função detectora de Zhou apresenta duas etapas para confirmar a existência da mesma. O detector de Hill [11] utiliza os indicadores de suavidade $\left(I S_{k}\right)$ do método WENO [13], sendo necessários vários passos para determinar se uma região apresenta função suave ou descontínua. Esses detectores de descontinuidades são detalhados no apêndice C.

Outro fator importante é a dependência de valores limiares, que variam conforme a função considerada, sendo necessário que um ajuste seja realizado de acordo com a função escolhida. Tanto as funções detectoras desenvolvidas por Adams, Pirozzoli, Zhou e Yee necessitam desse ajuste, enquanto que os detectores de Kim, Hill e o novo detector não necessitam dessa adaptação.

Esse estudo permitiu que um novo detector fosse desenvolvido com a finalidade de suprir as dificuldades em encontrar as regiões suaves e descontínuas de outros métodos. Além disso, essa nova função detectora tem como finalidade aproveitar as principais vantagens dos métodos de Adams [2], Yee [33], Kim [14], Hill [11], Pirozzoli [21] e Zhou [36].

A seguir será apresentada e detalhada essa nova função detectora para os casos unidimensional (1D) e bidimensional (2D), sendo este último uma extensão do caso 1D. Além disso, é apresentada uma breve explicação do método de diferenças centrais utilizado no cálculo do detector desenvolvido.

\subsection{Diferenças Centrais}

Para uma determinada função $f(x)$ e uma malha com espaçamento uniforme, $h=x_{j+1}-x_{j}$, essa função pode ser escrita tal que

$$
f\left(x_{j}\right)=f_{j}, j=0,1,2, \ldots, N_{x},
$$


sendo que a primeira derivada da função, $\frac{d f}{d x}\left(x_{j}\right)$, pode ser aproximada por ordem $2 n$ utilizandoses $2 n+1$ pontos $\left(x_{j-n}, \ldots, x_{j}, \ldots, x_{j+n}\right)$, além dos valores da função $\left(f_{j-n}, \ldots, f_{j}, \ldots, f_{j+n}\right)$.

Com isso, tem-se

$$
\frac{d f}{d x}\left(x_{j}\right)=\frac{1}{h} \sum_{k=-n}^{k=n} w_{k} f_{j+k},
$$

em que $w_{k}$ são os pesos Lagrangianos do cálculo da primeira derivada. Para determinar os valores para esses pesos é necessário expandir a equação (2.3) em série de Taylor ao redor de $f_{j}$, resultando no seguinte sistema

$$
\left[\begin{array}{ccccccccc}
1 & 1 & \ldots & 1 & 1 & 1 & \ldots & 1 & 1 \\
-n & (1-n) & \ldots & -1 & 0 & 1 & \ldots & (n-1) & n \\
(-n)^{2} & (1-n)^{2} & \ldots & (-1)^{2} & 0 & 1 & \ldots & (n-1)^{2} & n^{2} \\
\vdots & \vdots & \vdots & \vdots & \vdots & \vdots & \vdots & \vdots & \vdots \\
\vdots & \vdots & \vdots & \vdots & \vdots & \vdots & \vdots & \vdots & \vdots \\
\vdots & \vdots & \vdots & \vdots & \vdots & \vdots & \vdots & \vdots & \vdots \\
\vdots & \vdots & \vdots & \vdots & \vdots & \vdots & \vdots & \vdots & \vdots \\
(-n)^{2 n-3} & (1-n)^{2 n-3} & \ldots & (-1)^{2 n-3} & 0 & 1^{2 n-3} & \ldots & (n-1)^{2 n-3} & n^{2 n-3} \\
(-n)^{2 n-2} & (1-n)^{2 n-2} & \ldots & (-1)^{2 n-2} & 0 & 1^{2 n-2} & \ldots & (n-1)^{2 n-2} & n^{2 n-2} \\
(-n)^{2 n-1} & (1-n)^{2 n-1} & \ldots & (-1)^{2 n-1} & 0 & 1^{2 n-1} & \ldots & (n-1)^{2 n-1} & n^{2 n-1}
\end{array}\right]\left[\begin{array}{c}
w_{-n} \\
w_{-(n+1)} \\
w_{-(n+2)} \\
\vdots \\
w_{-1} \\
w_{0} \\
w_{1} \\
\vdots \\
w_{(n+2)} \\
w_{(n+1)} \\
w_{n}
\end{array}\right]=\left[\begin{array}{c}
0 \\
1 \\
0 \\
\vdots \\
0 \\
0 \\
0 \\
\vdots \\
0 \\
0 \\
0
\end{array}\right]
$$

Dessa forma, para um determinado $n$, monta-se o sistema (2.4) afim de obter os pesos Lagrangianos desejados, e consequentemente escrever a equação (2.3) completa.

Para o detector desenvolvido foi considerado o método de diferenças centrais de ordem 6 , ou seja, $n=3$. Assim, a equação (2.3) torna-se

$$
f_{j}^{\prime}=\frac{1}{h}\left(w_{-3} f_{j-3}+w_{-2} f_{j-2}+w_{-1} f_{j-1}+w_{0} f_{j}+w_{1} f_{j+1}+w_{2} f_{j+2}+w_{3} f_{j+3}\right),
$$

cujo sistema é dado por

$$
\left[\begin{array}{ccccccc}
1 & 1 & 1 & 1 & 1 & 1 & 1 \\
-3 & -2 & -1 & 0 & 1 & 2 & 3 \\
9 & 4 & 1 & 0 & 1 & 4 & 9 \\
-27 & -8 & -1 & 0 & 1 & 8 & 27 \\
81 & 16 & 1 & 0 & 1 & 16 & 81 \\
-243 & -32 & -1 & 0 & 1 & 32 & 243
\end{array}\right]\left[\begin{array}{c}
w_{-3} \\
w_{-2} \\
w_{-1} \\
w_{0} \\
w_{1} \\
w_{2} \\
w_{3}
\end{array}\right]=\left[\begin{array}{l}
0 \\
1 \\
0 \\
0 \\
0 \\
0 \\
0
\end{array}\right]
$$

Resolvendo o sistema (2.6), os pesos obtidos são

$$
w_{-3}=-\frac{1}{60}, w_{-2}=\frac{3}{20}, w_{-1}=-\frac{3}{4}, w_{0}=0, w_{1}=\frac{3}{4}, w_{2}=-\frac{3}{20}, w_{3}=\frac{1}{60} .
$$


Portanto, a equação (2.5) é reescrita como

$$
f^{\prime}\left(x_{j}\right)=f_{j}^{\prime}=\frac{1}{h}\left(-\frac{1}{60} f_{j-3}+\frac{3}{20} f_{j-2}-\frac{3}{4} f_{j-1}+\frac{3}{4} f_{j+1}-\frac{3}{20} f_{j+2}+\frac{1}{60} f_{j+3}\right) .
$$

A ordem de convergência é determinada através do cálculo do erro de truncamento. Com a expansão em série de Taylor, o erro de truncamento obtido é

$$
\begin{aligned}
\tau_{\text {Central }} & =-\frac{1}{140} h^{6} f^{(7)}-\frac{1}{720} h^{8} f^{(9)}-\frac{7}{52800} h^{10} f^{(11)}-\frac{1}{122850} h^{12} f^{(13)} \\
& -\frac{13}{36288000} h^{14} f^{(15)}+O\left(h^{16}\right)
\end{aligned}
$$

caracterizando um método de ordem 6. Como todas as derivadas são de ordem ímpar, o método de diferenças centrais é dispersivo.

\subsubsection{Análise de Fourier}

Para avaliar e obter mais informações sobre o método, é realizada uma análise de Fourier. Considerando-se a função $f(x)$ periódica, ou seja, $f_{1}=f_{N_{x}+1}$ e o domínio $[0, L]$ tal que $x_{1}=0$, $x_{N_{x}}=L$ e $h=L / N_{x}$, a função $f(x)$ em série de Fourier é

$$
f(x)=\sum_{k=-N_{x} / 2}^{k=N_{x} / 2} f_{k} \exp \left(\frac{2 \pi i k x}{L}\right), i=\sqrt{-1}
$$

Sem perda de generalidade, é possível considerar apenas um elemento $k$ do somatório apresentado na equação (2.10), sendo este

$$
F(x)=\exp \left(\frac{2 \pi i k x}{L}\right)
$$

Seja $x_{j}=(j-1) h$ e o número de onda dado por

$$
w=\frac{2 \pi k h}{L}=\frac{2 \pi k}{N_{x}}
$$

A expressão (2.11) calculado no ponto $x_{j}$ é tal que

$$
F_{j}=\exp \left(\frac{2 \pi i k j}{N_{x}}\right)=\exp (i w j)
$$

Da mesma forma calcula-se $F(x)$ para o ponto $x_{j+l}$, cuja expressão é

$$
F_{j+l}=\exp \left(\frac{2 \pi i k(j+l)}{N_{x}}\right)=\exp \left(\frac{2 \pi i k l}{N_{x}}\right) F_{j}=\exp (i w l) F_{j}
$$


Assim, é possível determinar as derivadas em relação a $x$ das equações (2.13) e (2.14) através da regra da cadeia,

$$
F_{j}^{\prime}=\frac{i w^{*}}{h} F_{j}
$$

$\mathrm{e}$

$$
F_{j+l}^{\prime}=\frac{i w^{*}}{h} F_{j} \exp (i w l) .
$$

Observa-se que $w^{*}$ representa o número de onda modificado, sendo essa mudança causada pelo cálculo da derivada de $F(x)$. Com isso, substituindo as equações (2.13), (2.14), (2.15) e (2.16) na expressão (2.8) que determina o método central de ordem 6, tem-se

$$
w^{*}(w)=\frac{1}{30}[45 \operatorname{sen}(w)-9 \operatorname{sen}(2 w)+\operatorname{sen}(3 w)]
$$

que determina $w^{*}$ em função de $w$.

A Figura 2.1 mostra o gráfico de $w^{*}$ em função de $w$ obtido através da equação (2.17).

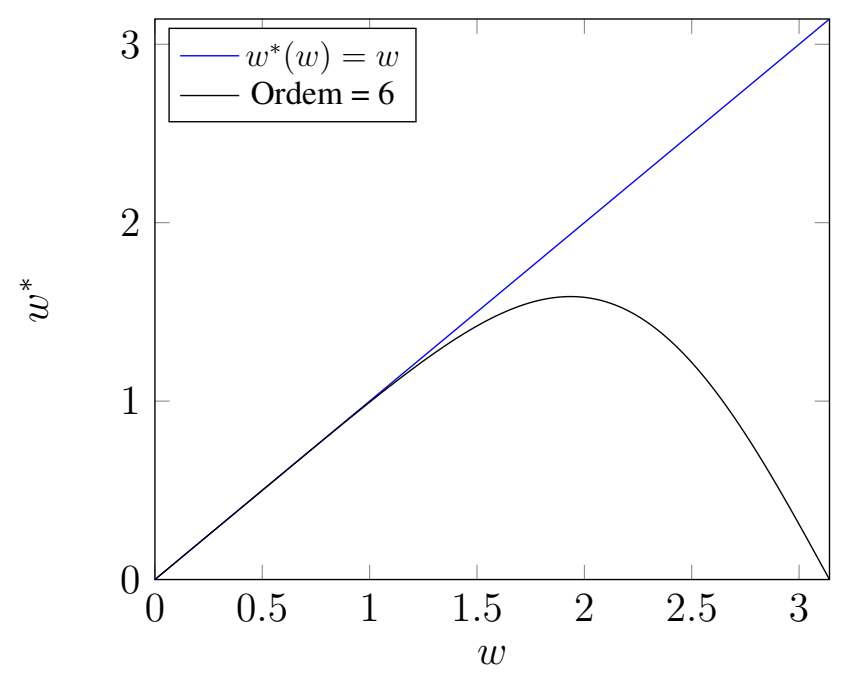

Parte Real

Figura 2.1: Análise de Fourier - Diferenças Centrais

Nota-se que a reta representa a função descontínua em que $w^{*}(\pi)=0$ e $w^{*}(w)=w$, para $w \neq \pi$; já a curva representa a função para o esquema de diferenças centrais de ordem 6 . Como a equação (2.17) não apresenta parte imaginária, pode-se afirmar que o método é não dissipativo.

\subsection{Caso Unidimensional}

Através das diversas ideias apresentadas por outros métodos numéricos híbridos existentes e estudados, a fim de construir o indicador de suavidade para uma determinada função $f(x)$, foi considerado o método de diferenças centrais de ordem 6 (2.8).

Com a equação (2.8) e o cálculo da derivada de uma função $f(x)$, é possível definir o indicador de suavidade $r_{i}$ para avaliar se uma determinada região pode ser considerada como função suave 
ou descontínua. Para construir esse indicador é considerada uma malha com espaçamento $h$ entre dois pontos consecutivos, denominada de malha mais fina, e uma outra malha com espaçamento $2 h$ entre dois pontos consecutivos, denominada de malha mais grossa. Essa última apresenta a metade do número de pontos da primeira.

O indicador de suavidade é definido através da seguinte razão:

$$
r_{i}=\frac{(2 h)^{4} f_{2 h, i}^{(4)}+(2 h)^{5} f_{2 h, i}^{(5)}+(2 h)^{6} f_{2 h, i}^{(6)}}{h^{4} f_{h, i}^{(4)}+h^{5} f_{h, i}^{(5)}+h^{6} f_{h, i}^{(6)}+\varepsilon}
$$

com $f_{2 h, i}^{(n)}$ sendo a $n$-ésima derivada numérica de $f$ na malha mais grossa, $f_{h, i}^{(n)}$ a $n$-ésima derivada numérica na malha mais fina e uma constante $\varepsilon$ para evitar uma possível divisão por zero.

Observa-se na equação (2.18) que o indicador $r_{i}$ envolve uma razão entre derivadas calculadas nas malhas mais grossa e mais fina. Para calcular essa razão, é realizada uma combinação com os valores das derivadas de ordem 4,5 e 6 de ambas as malhas.

Para o cálculo dessas derivadas é utilizado o método de diferenças finitas apresentado na equação (2.8), sendo que dada uma determinada função $f(x)$, calcula-se primeiramente a derivada $f^{\prime}(x)$ para os pontos de ambas as malhas. Com os valores da derivada em cada ponto, o método é aplicado novamente com a intenção de obter a derivada segunda $f^{\prime \prime}(x)$ da função especificada. Repetindo o processo diversas vezes, é possível calcular os valores das derivadas de ordem 4,5 e 6 , respectivamente.

Pela equação (2.18) é possível observar que se em uma determinada região a função $f(x)$ considerada for constante, o indicador de suavidade $r_{i}$ não será calculado corretamente, já que na região que de fato é suave o valor não será próximo de $2^{4}=16$. Assim, para evitar esse tipo de problema é somada uma pequena parcela da função $\operatorname{sen}(\pi x)$, fazendo com que a região constante seja determinada corretamente e não afetando também as outras regiões, inclusive as que apresentam descontinuidades.

A fim de facilitar os cálculos e obter as regiões descontínuas corretamente, a função $f(x)$ é normalizada em $[0,1]$ :

$$
f_{\text {norm }, i}=\frac{f_{i}-f_{\min }}{f_{\max }-f_{\min }} .
$$

Caso $f_{\min }=f_{\max }(f$ constante $)$, o cálculo da função normalizada é alterado

$$
f_{\text {norm }, i}=f_{i}-f_{\text {min }}
$$

Nota-se que os valores $f_{\min }$ e $f_{\max }$ representam o menor e maior valor da função $f(x)$ em todos os pontos da malha, respectivamente. Dessa forma, pelas equações (2.19) e (2.20) é possível determinar em cada ponto o valor da função $f(x)$ normalizada. 
Os pesos $(\sigma)$ que indicam se determinada região é suave ou descontínua são definidos da seguinte forma:

$$
\sigma_{i}=\left\{\begin{array}{ll}
1, & \text { se } r_{i} \geq \hat{r} \\
0, & \text { caso contrário }
\end{array},\right.
$$

em que $\hat{r}$ é um valor limiar com $\sigma_{i}=1$ indicando uma região com função suave e $\sigma_{i}=0$ indicando uma região com função descontínua.

\subsubsection{Algoritmo}

O procedimento para o novo detector é resumido no Algoritmo 1.

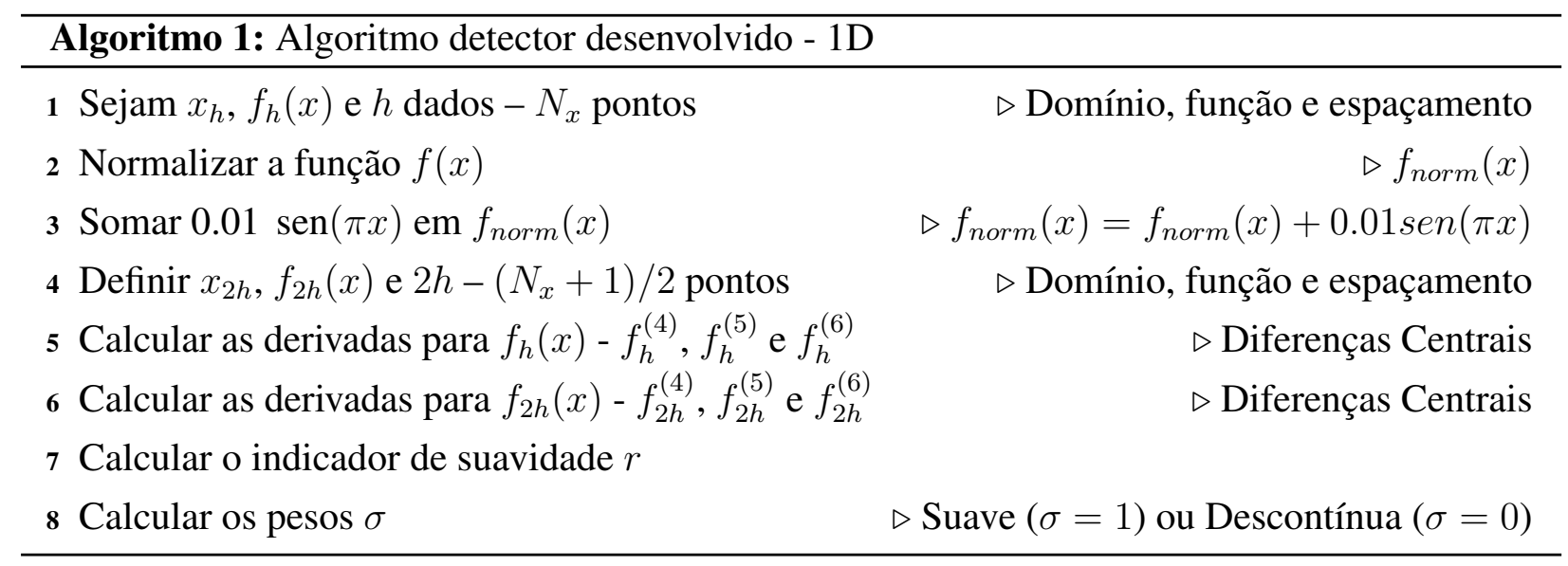

\subsection{Caso Bidimensional}

Para o caso bidimensional, a função considerada depende das variáveis independentes $x$ e $y$ tal que $f(x, y)$. Assim como no caso unidimensional, a função pode ser contínua com derivadas contínuas ou apresentar descontinuidades na própria função ou em suas derivadas.

Dessa forma, a ideia do novo detector de descontinuidades é determinar quais regiões dessa função podem ser consideradas como suaves e quais podem ser consideradas como descontínuas. A aplicação da função detectora para o caso 2D ocorre de forma muito similar à aplicada para o caso unidimensional, sendo necessária a aplicação em cada direção, ou seja, nas direções $x$ e $y$.

O indicador de suavidade apresentado na equação (2.18) é aplicado primeiramente na direção $x$, ou seja, em todas as linhas da função que compõem a função $f(x, y)$, sendo então posteriormente aplicada a equação (2.21) para determinar os pesos $\sigma_{x}$ em cada ponto da malha, resultando em uma matriz com as dimensões do domínio considerado. O mesmo ocorre na direção $y$, sendo estas equações aplicadas em cada coluna da função e determinando assim uma matriz de pesos $\sigma_{y}$.

Com as matrizes $\sigma_{x}$ e $\sigma_{y}$ definidas, é possível afirmar quais regiões são suaves e quais apresentam descontinuidades em ambas as direções. 


\subsection{Observações}

Através do desenvolvimento do novo detector observa-se que para determinar os pesos $\sigma$ que indicam se uma determinada região apresenta função suave ou descontínua, é necessário definir um valor limiar $\hat{r}$ apresentado na equação (2.21).

Em geral, esse valor limiar deve ser determinado pelo usuário, ou seja, o mesmo deve ser ajustado dependendo do tipo de função considerada. Porém, com a análise realizada no próximo capítulo é possível observar um padrão que facilita a escolha de $\hat{r}$ e que funciona para diversos tipos de funções, determinando as regiões corretamente. 
O detector de descontinuidades (2.21) contruído no capítulo anterior depende de um valor limiar assim como os detectores apresentados por Adams [2], Pirozzoli [21], Zhou [36] e Yee [33]. Porém, o novo detector apresenta um padrão para o indicador de suavidade (2.8) que pode ser utilizado para definir $\hat{r}$.

Para determinar sistematicamente o valor limiar $\hat{r}$, será apresentada uma análise do indicador de suavidade $r_{i}$. Afim de que essa análise seja completa e detalhada, são construídos polinômios de grau $N \leq N_{x}$ para funções $f(x)$ suave e com descontinuidade em uma de suas derivadas através da interpolação de Lagrange. Com os polinômios obtidos, estes são analisados afim de observar e discutir a relação que ambos possuem [18].

Com a relação e os polinômios obtidos é possível analisar a razão (2.18) determinada para o novo detector desenvolvido, tanto para o caso em que a função e suas derivadas são contínuas como para o caso em que a função ou uma de suas derivadas apresenta descontinuidade.

\subsection{Construção de Polinômios}

Com os valores discretos de uma função $f(x)$ nos nós de uma malha uniforme $x_{i}, i=0,1, \ldots, N$ com espaçamento $h=x_{i+1}-x_{i}$, o objetivo é localizar a região onde essa função ou uma de suas derivadas (até ordem 3 ) possui descontinuidade.

Primeiramente será definido o polinômio $p_{h}(x)$ para uma função suave, cuja função e suas derivadas são contínuas. Em seguida, o polinômio $P_{h}(x)$ é definido para o caso em que a função e/ou suas derivadas apresentam descontinuidades (saltos) $S_{m}$. A ideia é mostrar que os polinômios 
$P_{h}(x)$ e $p_{h}(x)$ variam apenas por uma combinação dos saltos $S_{m}$. E por fim, essa informação será utilizada para aproximar a razão do detector e mostrar que, com uma função não suave, essa é aproximadamente $2^{n}$ com salto a partir da $n$-ésima derivada, enquanto que uma função suave possui razão $2^{4}=16$.

\subsubsection{Polinômio para Função Suave}

O polinômio de interpolação de grau $N$ para uma função $f(x)$ suave, ou seja, que não possui descontinuidades (saltos) na função ou em suas derivadas é definido tal que:

$$
\begin{gathered}
p_{h}\left(x_{i}\right)=f\left(x_{i}\right)=f_{i}, i=0, \ldots, N \\
p_{h}(x)=\sum_{i=0}^{N} f_{i} L_{i}(x), L_{i}(x)=\prod_{j=0}^{N} \frac{x-x_{j}}{x_{i}-x_{j}}, i \neq j .
\end{gathered}
$$

Isto é, o polinômio de interpolação de Lagrange é construído assumindo que $f(x)$ é uma função suave, e sua $m$-ésima derivada é dada por

$$
p_{h}^{(m)}(x)=\sum_{i=0}^{N} f_{i} L_{i}^{(m)}(x), m=0,1, \ldots, N .
$$

Dessa forma, a $m$-ésima derivada de $f(x)$ em $x_{J} \in\left[x_{0}, x_{N}\right]$ é tal que

$$
f^{(m)}\left(x_{J}\right) \approx p_{h}^{(m)}\left(x_{J}\right)=\sum_{i=0}^{N} f_{i} L_{i}^{(m)}\left(x_{J}\right), m=0,1, \ldots, N
$$

\subsubsection{Polinômio para Função Não Suave}

Supondo que a função $f(x)$ não é suave, ou seja, sua $m$-ésima derivada ou a própria função possui uma descontinuidade (salto) $S_{m}$ em $x=\xi$, tem se que

$$
f^{(m)}\left(\xi^{+}\right)-f^{(m)}\left(\xi^{-}\right)=S_{m}, \xi \in\left[x_{0}, x_{N}\right], 0 \leq m \leq N
$$

Assim, o polinômio interpolador $p_{h}(x)$ não representa corretamente a função $f(x)$ no intervalo $\left[x_{0}, x_{N}\right]$, sendo então necessário incorporar o salto $S_{m}$ na construção do novo polinômio $P_{h}(x)$ de grau $N[18]$ tal que

$$
P_{h}(x)=\theta(x-\xi) P_{h,+}(x)+\theta(\xi-x) P_{h,-}(x),
$$

em que

$$
\theta(x)= \begin{cases}1, & x>0 \\ 1 / 2, & x=0 \\ 0, & x<0\end{cases}
$$


e

$$
P_{h,+}(x)=\sum_{i=0}^{N} c_{i}^{+}(\xi) L_{i}(x), P_{h,-}(x)=\sum_{i=0}^{N} c_{i}^{-}(\xi) L_{i}(x) .
$$

Asssumindo que

$$
\begin{gathered}
P_{h}\left(x_{i}\right)=f_{i}, i=0,1, \ldots, N \\
P_{h,+}^{(m)}(\xi)-P_{h,-}^{(m)}(\xi)=S_{m}, m=0,1, \ldots, N
\end{gathered}
$$

e substituindo nas definições anteriores, obtém-se

$$
S_{m}=P_{h,+}^{(m)}(\xi)-P_{h,-}^{(m)}(\xi)=\sum_{i=0}^{N}\left[c_{i}^{+}(\xi)-c_{i}^{-}(\xi)\right] L_{i}^{(m)}(\xi), m=0,1, \ldots, N
$$

Nota-se na equação (3.11) que um sistema linear tem que ser resolvido para $i=0,1, \ldots, N$ e assim determinar $c_{i}^{+}(\xi)$ e $c_{i}^{-}(\xi)$. Resolvendo o sistema linear, obtém-se que

$$
c_{i}^{+}(\xi)-c_{i}^{-}(\xi)=\sum_{r=0}^{N} \frac{\left(x_{i}-\xi\right)^{r}}{r !} S_{r}
$$

Através das relações, tem-se então

$$
\begin{aligned}
& c_{i}^{+}(\xi)=f_{i}+\theta\left(\xi-x_{i}\right) \sum_{r=0}^{N} \frac{\left(x_{i}-\xi\right)^{r}}{r !} S_{r}, \\
& c_{i}^{-}(\xi)=f_{i}+\theta\left(x_{i}-\xi\right) \sum_{r=0}^{N} \frac{\left(x_{i}-\xi\right)^{r}}{r !} S_{r} .
\end{aligned}
$$

Com isso, o polinômio $P_{h}(x)$ para uma função $f(x)$ não suave é dado por

$$
P_{h}(x)=p_{h}(x)+\sum_{i=0}^{N}\left[\theta(x-\xi) \theta\left(\xi-x_{i}\right)-\theta(\xi-x) \theta\left(x_{i}-\xi\right)\right] L_{i}(x) \sum_{r=0}^{N} \frac{\left(x_{i}-\xi\right)^{r}}{r !} S_{r},
$$

cuja $m$-ésima derivada em $x=\xi$ é

$$
P_{h}^{(m)}(\xi)=p_{h}^{(m)}(\xi)+\frac{S_{m}}{2}-\sum_{r=0}^{N}\left[\sum_{i=0}^{N} \theta\left(x_{i}-\xi\right) L_{i}^{(m)}(\xi)\left(x_{i}-\xi\right)^{r}\right] \frac{S_{r}}{r !}
$$

\subsection{Análise}

Com os polinômios $p_{h}(x)$ para uma função suave e $P_{h}(x)$ para uma função não suave definidos, é realizada uma análise mais detalhada da razão definida na equação (2.18) durante o desenvolvimento do novo detector. 


\subsubsection{Função Não suave}

Para simplificar a análise, suponha que $\xi=x_{J}$, em que $x_{J}$ é um dos nós da malha, $J \in \mathbb{Z}$, $0 \leq J \leq N$, com $0 \leq m \leq N$. Assim, $P_{h}^{(m)}(\xi)$ será reescrito e sua forma analisada:

$$
\begin{aligned}
P_{h}^{(m)}\left(x_{J}\right) & =p_{h}^{(m)}\left(x_{J}\right)+\frac{S_{m}}{2}-\sum_{r=0}^{N}\left[\sum_{i=0}^{N} \theta\left(x_{i}-x_{J}\right) L_{i}^{(m)}\left(x_{J}\right)\left(x_{i}-x_{J}\right)^{r}\right] \frac{S_{r}}{r !} \\
& =p_{h}^{(m)}\left(x_{J}\right)+\frac{S_{m}-L_{J}^{(m)}\left(x_{J}\right) S_{0}}{2}-\sum_{r=0}^{N}\left[\sum_{i=J+1}^{N} L_{i}^{(m)}\left(x_{J}\right)(i-J)^{r}\right] \frac{h^{r} S_{r}}{r !}
\end{aligned}
$$

sendo

$$
L_{i}^{(m)}\left(x_{J}\right)=\frac{\alpha(i, J, m)}{h^{m}}
$$

em que $\alpha(i, J, m)$ é constante real e não depende de $h \mathrm{e}$

$$
\alpha(i, J, 0)=\left\{\begin{array}{l}
1, i=J \\
0, i \neq J
\end{array}\right.
$$

Com isso, a equação (3.17) pode ser reescrita da seguinte forma:

$$
\begin{aligned}
P_{h}^{(m)}\left(x_{J}\right) & =p_{h}^{(m)}\left(x_{J}\right)+\frac{h^{m} S_{m}-\alpha(J, J, m) S_{0}}{2 h^{m}}-\sum_{r=0}^{N}\left[\sum_{i=J+1}^{N} \alpha(i, J, m) \frac{(i-J)^{r}}{r !}\right] \frac{h^{r} S_{r}}{h^{m}} \\
& =p_{h}^{(m)}\left(x_{J}\right)+\frac{1}{h^{m}} \sum_{r=0}^{N} \beta(r, J, m) h^{r} S_{r},
\end{aligned}
$$

sendo que

$$
\beta(r, J, m)=\frac{\delta_{r m}-\alpha(J, J, m) \delta_{r 0}}{2}-\sum_{i=J+1}^{N} \frac{\alpha(i, J, m)(i-J)^{r}}{r !}
$$

com

$$
\delta_{i j}=\left\{\begin{array}{ll}
1, & i=j \\
0, & i \neq j
\end{array} .\right.
$$

\subsubsection{Soma de Derivadas}

Seja $R_{h}$ a soma de termos de ordem 4,5 e 6 para $\xi=x_{J}$, em que $0 \leq J \leq N$, tal que

$$
R_{h}\left(x_{J}\right)=h^{4} P_{h}^{(4)}\left(x_{J}\right)+h^{5} P_{h}^{(5)}\left(x_{J}\right)+h^{6} P_{h}^{(6)}\left(x_{J}\right)
$$


A equação (3.21) pode ser reescrita utilizando-se a equação (3.19) da seguinte forma:

$$
\begin{aligned}
& R_{h}\left(x_{J}\right)= \\
& =h^{4}\left[p_{h}^{(4)}\left(x_{J}\right)+\frac{1}{h^{4}} \sum_{r=0}^{N} \beta(r, J, 4) h^{r} S_{r}\right]+h^{5}\left[p_{h}^{(5)}\left(x_{J}\right)+\frac{1}{h^{5}} \sum_{r=0}^{N} \beta(r, J, 5) h^{r} S_{r}\right]+ \\
& +h^{6}\left[p_{h}^{(6)}\left(x_{J}\right)+\frac{1}{h^{6}} \sum_{r=0}^{N} \beta(r, J, 6) h^{r} S_{r}\right]= \\
& =\left[h^{4} p_{h}^{(4)}\left(x_{J}\right)+h^{5} p_{h}^{(5)}\left(x_{J}\right)+h^{6} p_{h}^{(6)}\left(x_{J}\right)\right]+\sum_{r=0}^{N}[\beta(r, J, 4)+\beta(r, J, 5)+\beta(r, J, 6)] h^{r} S^{r} .
\end{aligned}
$$

\subsubsection{Razão do Detector Desenvolvido}

Como $\beta$ não depende diretamente de $h$ e sendo $p_{h}^{(m)}(\xi) \approx p_{2 h}^{(m)}(\xi)$, desde que o número de pontos utilizados na interpolação sejam equidistantes, tem-se

$$
r(\xi)=\frac{R_{2 h}(\xi)}{R_{h}(\xi)}=\frac{\sum_{l=4}^{6}\left[(2 h)^{l} p_{2 h}^{(l)}(\xi)\right]+\sum_{r=0}^{N}\left[(2 h)^{r} S_{r} \sum_{l=4}^{6} \beta(r, J, l)\right]}{\sum_{l=4}^{6}\left[h^{l} p_{h}^{(l)}(\xi)\right]+\sum_{r=0}^{N}\left[h^{r} S_{r} \sum_{l=4}^{6} \beta(r, J, l)\right]} .
$$

Quando uma função $f(x)$ e suas derivadas não possuem descontinuidades, ou seja,

$$
S_{r}=0, r=0,1, \ldots, N
$$

a equação (3.23) é reescrita tal que

$$
\begin{aligned}
r(\xi) & =\frac{(2 h)^{4} p_{2 h}^{(4)}(\xi)+(2 h)^{5} p_{2 h}^{(5)}(\xi)+(2 h)^{6} p_{2 h}^{(6)}(\xi)}{h^{4} p_{h}^{(4)}(\xi)+h^{5} p_{h}^{(5)}(\xi)+h^{6} p_{h}^{(6)}(\xi)}= \\
& =\frac{(2 h)^{4}}{h^{4}} \frac{\left(p_{2 h}^{(4)}(\xi)+(2 h) p_{2 h}^{(5)}(\xi)+(2 h)^{2} p_{2 h}^{(6)}(\xi)\right.}{p_{h}^{(4)}(\xi)+h p_{h}^{(5)}(\xi)+h^{2} p_{h}^{(6)}(\xi)}= \\
& =2^{4} \times \frac{\left(p_{2 h}^{(4)}(\xi)+(2 h) p_{2 h}^{(5)}(\xi)+(2 h)^{2} p_{2 h}^{(6)}(\xi)\right.}{p_{h}^{(4)}(\xi)+h p_{h}^{(5)}(\xi)+h^{2} p_{h}^{(6)}(\xi)} \sim 2^{4}=16 .
\end{aligned}
$$

Porém, quando $f(x)$ possui uma descontinuidade (salto) na $n$-ésima derivada ( $n \leq 3)$, ou seja,

$$
\begin{aligned}
& S_{r}=0, r=0,1, \ldots, n-1, \\
& S_{n} \neq 0,
\end{aligned}
$$


a equação (3.23) é reescrita tal que

$$
\begin{aligned}
r(\xi) & =\frac{\sum_{l=4}^{6}\left[(2 h)^{l} p_{2 h}^{(l)}(\xi)\right]+\sum_{r=n}^{N}\left[(2 h)^{r} S_{r} \sum_{l=4}^{6} \beta(r, J, l)\right]}{\sum_{l=4}^{6}\left[h^{l} p_{h}^{(l)}(\xi)\right]+\sum_{r=n}^{N}\left[h^{r} S_{r} \sum_{l=4}^{6} \beta(r, J, l)\right]} \\
& =2^{n} \times \frac{\sum_{l=4}^{6}\left[(2 h)^{l-n} p_{2 h}^{(l)}(\xi)\right]+\sum_{r=n}^{N}\left[(2 h)^{r-n} S_{r} \sum_{l=4}^{6} \beta(r, J, l)\right]}{\sum_{l=4}^{6}\left[h^{l-n} p_{h}^{(l)}(\xi)\right]+\sum_{r=n}^{N}\left[h^{r-n} S_{r} \sum_{l=4}^{6} \beta(r, J, l)\right]} .
\end{aligned}
$$

Como $0 \leq n \leq 3$, tem-se que $1 \leq 4-n \leq 4$, e

$$
\begin{aligned}
r(\xi) & =2^{n} \times \frac{\sum_{t=0}^{N-n}\left[(2 h)^{t} S_{t+n} \sum_{l=4}^{6} \beta(t+n, J, l)\right]+\sum_{l=4}^{6}\left[(2 h)^{l-n} p_{2 h}^{l}(\xi)\right]}{\sum_{t=0}^{N-n}\left[h^{t} S_{t+n} \sum_{l=4}^{6} \beta(t+n, J, l)\right]+\sum_{l=4}^{6}\left[h^{l-n} p_{h}^{l}(\xi)\right]} \\
& =2^{n} \times \frac{\left[S_{n} \sum_{l=4}^{6} \beta(n, J, l)+O(2 h)\right]+O\left((2 h)^{4-n}\right)}{\left[S_{n} \sum_{l=4}^{6} \beta(n, J, l)+O(h)\right]+O\left(h^{4-n}\right)} \sim 2^{n},
\end{aligned}
$$

mostrando que, para funções que possuem descontinuidade na $n$-ésima derivada, com $0 \leq n \leq 3$, $r(\xi) \sim 2^{n}<2^{4}=16$.

\subsection{Exemplos}

Para mostrar a relação $2^{n}$ através de gráficos e observar os resultados obtidos para diferentes situações, foram consideradas diversas funções com características distintas. Assim, a relação $2^{n}$ é exemplificada para funções contínuas com derivadas contínuas e casos em que a primeira, segunda ou terceira derivadas apresentam descontinuidades.

As funções unidimensionais consideradas foram:

- Função Cosseno:

$$
f(x)=\cos (\pi x), \quad x \in[-1,1]
$$

- Função Mesa:

$$
f(x)=\left\{\begin{array}{ll}
1, & \text { se }-\frac{1}{5} \leq x \leq \frac{1}{5} \\
0, & \text { caso contrário }
\end{array}, \quad x \in[-1,1]\right.
$$

- Função Seno Descontínuo:

$$
f(x)=\left\{\begin{array}{ll}
\operatorname{sen}(3 \pi x)+2.5, & \text { se }-\frac{1}{5} \leq x \leq \frac{1}{5} \\
\operatorname{sen}(3 \pi x), & \text { caso contrário }
\end{array}, \quad x \in[-1,1]\right.
$$


- Função Módulo:

$$
f(x)=\left\{\begin{array}{ll}
x, & \text { se } x>0 \\
-x, & \text { se } x<0
\end{array}, \quad x \in[-1,1]\right.
$$

- Função Segunda Derivada Descontínua:

$$
f(x)=\left\{\begin{array}{ll}
x-x^{2} / 2, & \text { se } x>0 \\
x+x^{2} / 2, & \text { se } x<0
\end{array}, \quad x \in[-1,1]\right.
$$

- Função Terceira Derivada Descontínua:

$$
f(x)=\left\{\begin{array}{ll}
x^{3}, & \text { se } x>0 \\
-x^{3}, & \text { se } x<0
\end{array}, \quad x \in[-1,1]\right.
$$

- Função Frequência Alta:

$$
f(x)=\sin \left(\frac{\left(N_{x}-1\right) \pi x}{7}\right)+\sin \left(\frac{\left(N_{x}-1\right) \pi x}{9}\right), \quad x \in[-1,1] .
$$

\subsubsection{Cosseno}

A Figura 3.1 mostra os valores do parâmetro $r$ para a função Cosseno (3.29) com 81 pontos na discretização.

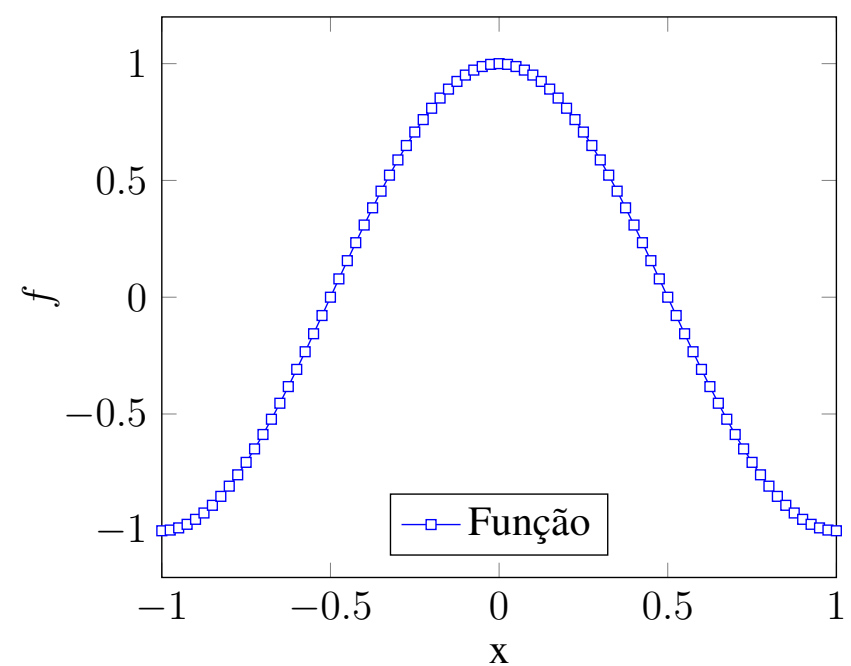

(a) Função - 81 pontos

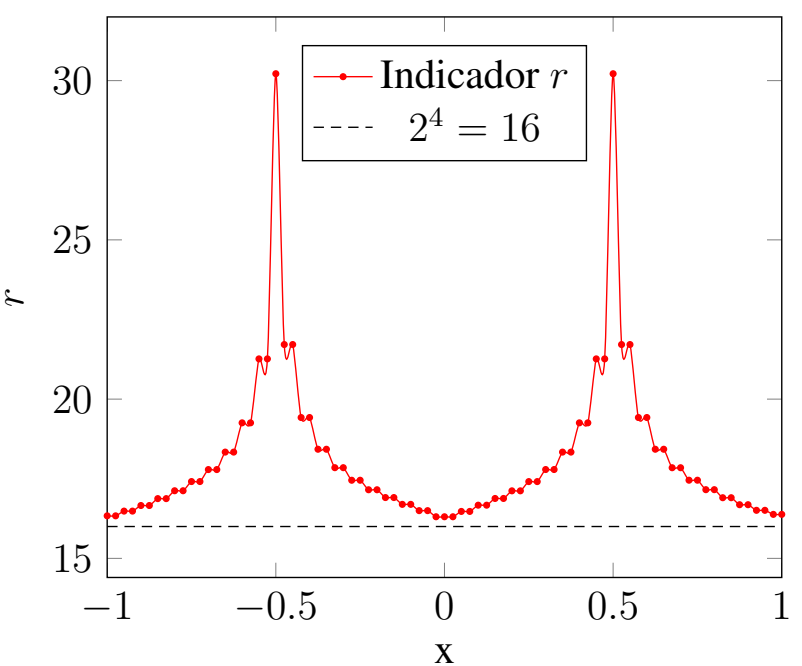

(b) Indicador - 81 pontos

Figura 3.1: Relação $2^{n}$ - Função Cosseno

Nota-se na Figura 3.1(b) que os valores obtidos para o indicador $r$ são maiores ou próximos a 16. Isso acontece já que a função Cosseno e suas derivadas são contínuas, resultando assim na relação apresentada na equação (3.25). 


\subsubsection{Mesa}

A Figura 3.2 mostra a relação $2^{n}$ para a função Mesa (3.30) que apresenta descontinuidade na própria função, resultando assim em valores diferentes do indicador $r$ nesses pontos.

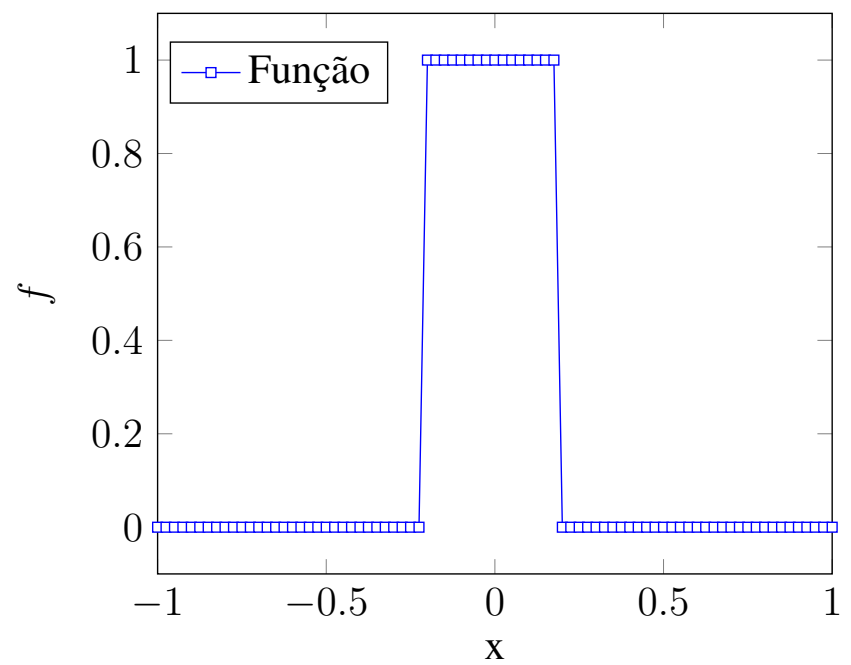

(a) Função - 81 pontos

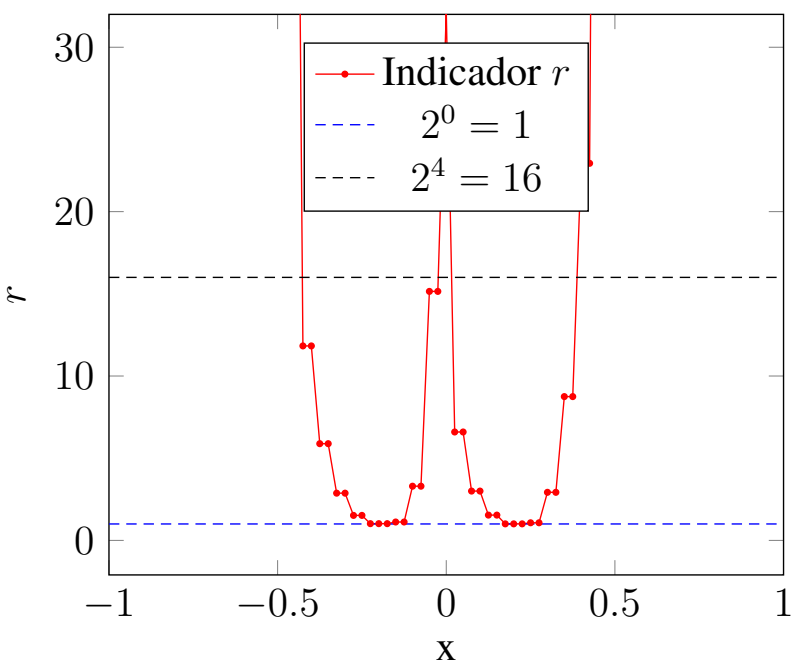

(b) Indicador - 81 pontos

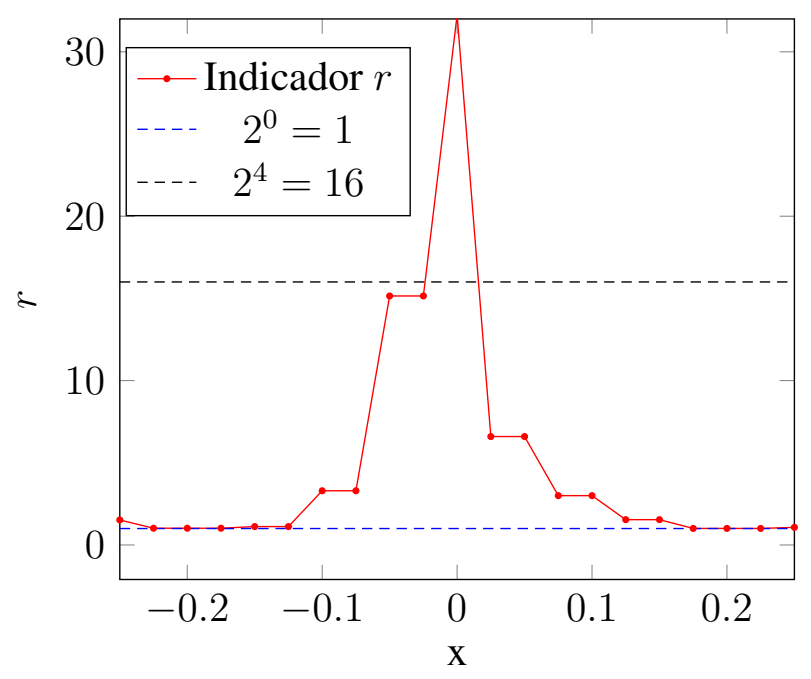

(c) Indicador - Ampliação - 81 pontos

Figura 3.2: Relação $2^{n}$ - Função Mesa

Observa-se pela Figura 3.2(b) nas regiões em que a função apresenta descontinuidade os valores obtidos para o indicador $r$ são muito próximos de $2^{0}=1$, evidenciando assim a relação $2^{n}$ discutida anteriormente e apresentada na equação (3.28). A Figura 3.2(c) mostra uma ampliação nas regiões que a função apresenta descontinuidade, sendo possível notar com mais clareza a relação $2^{n}$. 


\subsubsection{Seno Descontínuo}

A Figura 3.3 mostra o resultado obtido para o indicador $r$ com a função Seno Descontínuo (3.31), sendo esta muito similiar a função Mesa, já que a própria função apresenta descontinuidade em $x=-1 / 5$ e $x=1 / 5$. Para a discretização foram considerados 81 pontos.

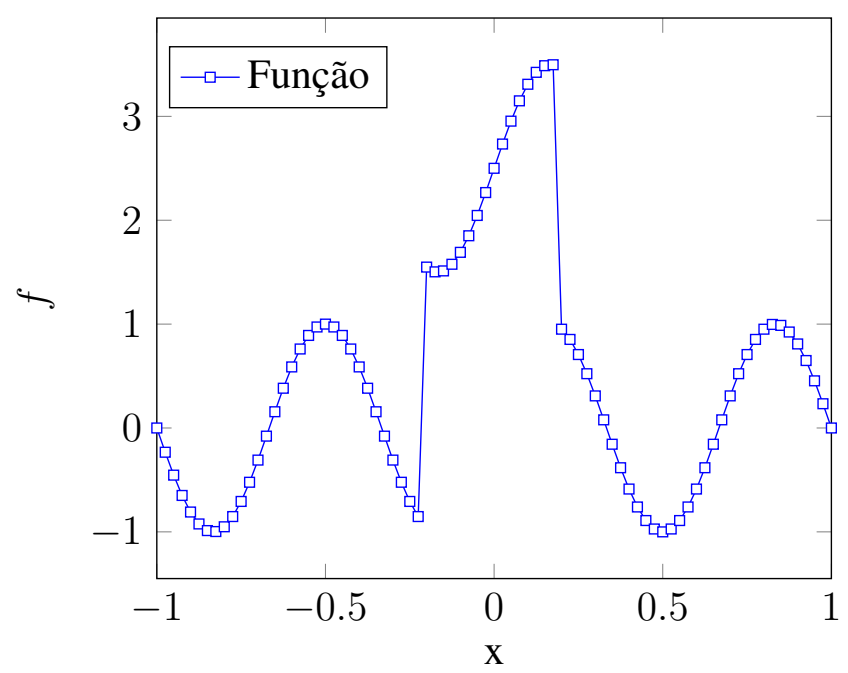

(a) Função - 81 pontos

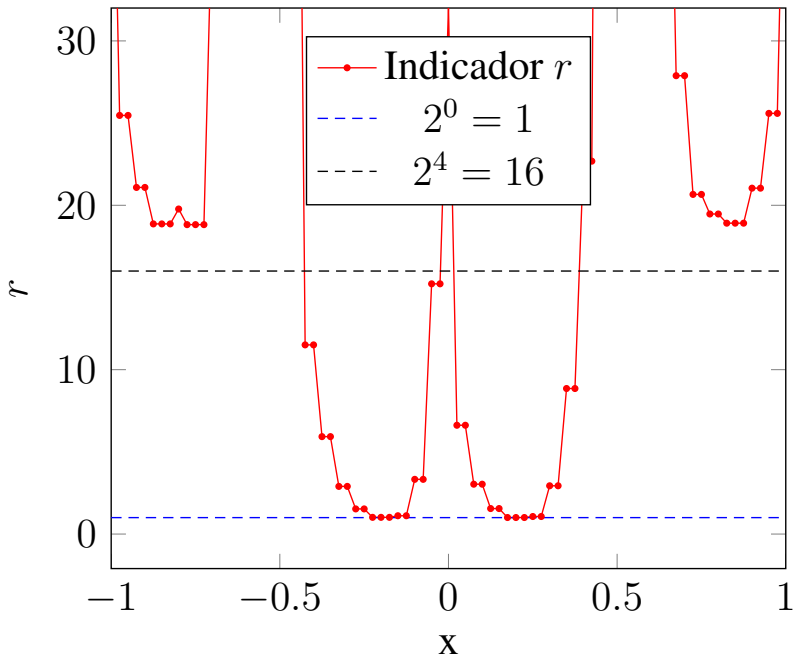

(b) Indicador - 81 pontos

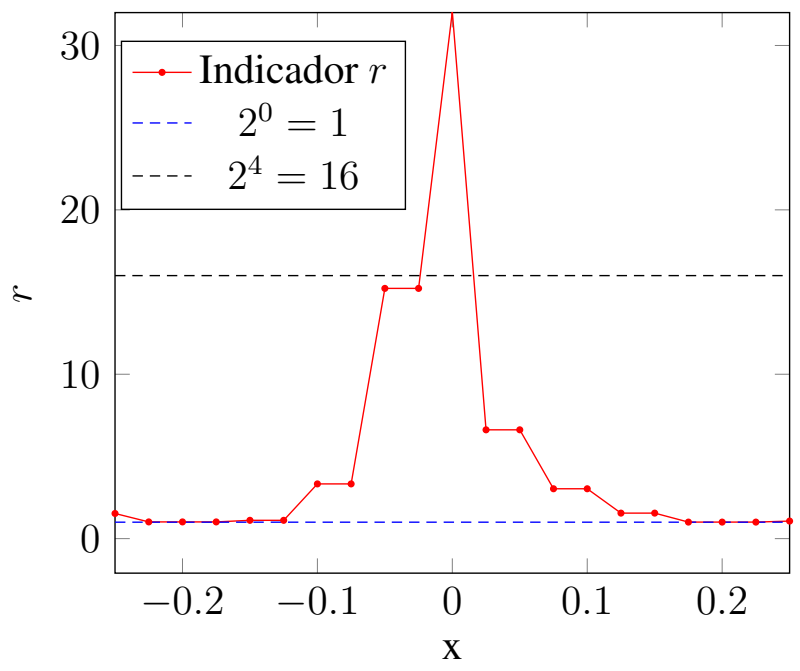

(c) Indicador - Ampliação - 81 pontos

Figura 3.3: Relação $2^{n}$ - Seno Descontínuo

Como é possível observar pelas Figuras 3.3(b) e 3.3(c), os valores para o indicador $r$ nas regiões suaves são maiores ou próximos a 16. Já nas regiões descontínuas os valores observados são próximos de $2^{0}=1$, indicando assim uma descontinuidade presente na função. 


\subsubsection{Módulo}

A figura 3.4 mostra a função Módulo (3.32) e o resultado obtido para o indicador $r$ e a relação $2^{n}$. Essa função apresenta uma descontinuidade na primeira derivada em $x=0$.

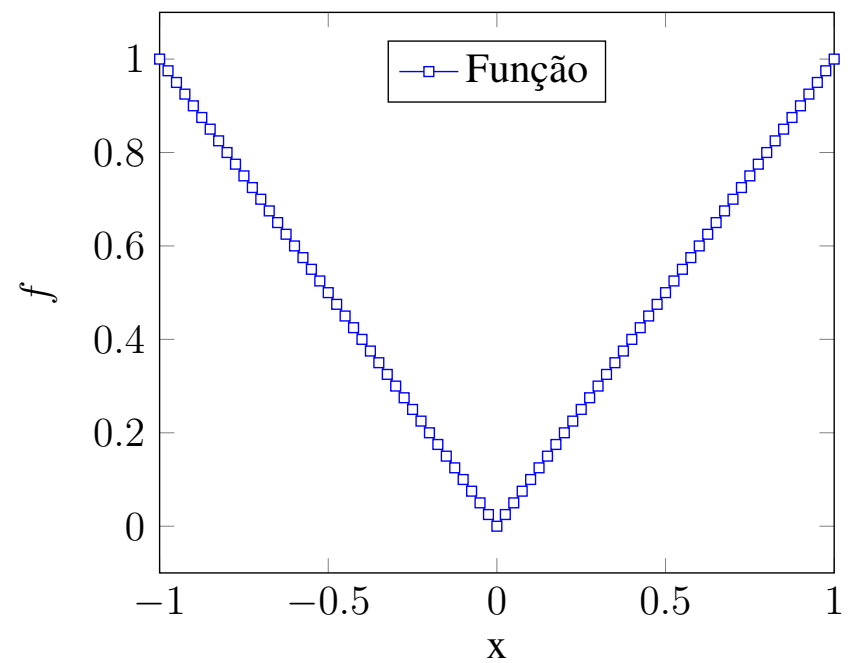

(a) Função - 81 pontos

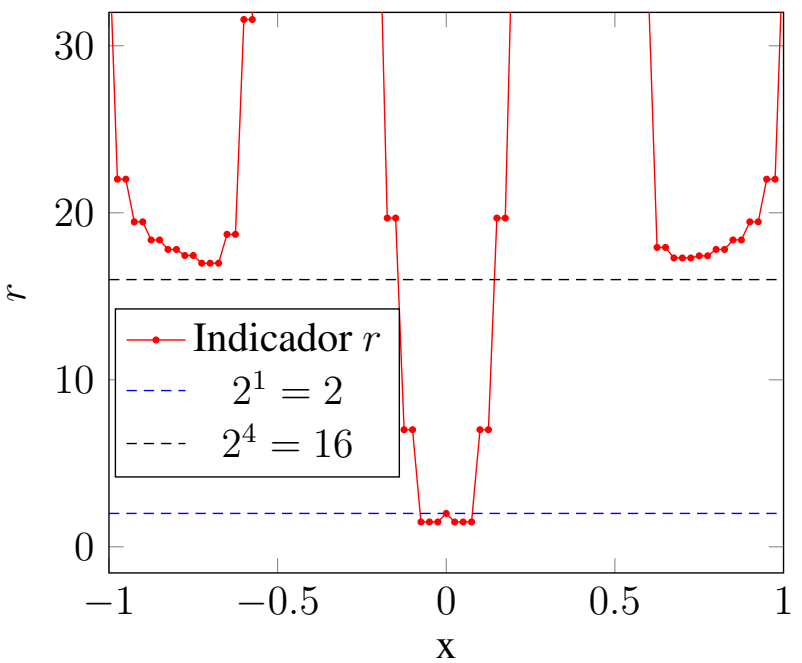

(b) Indicador - 81 pontos

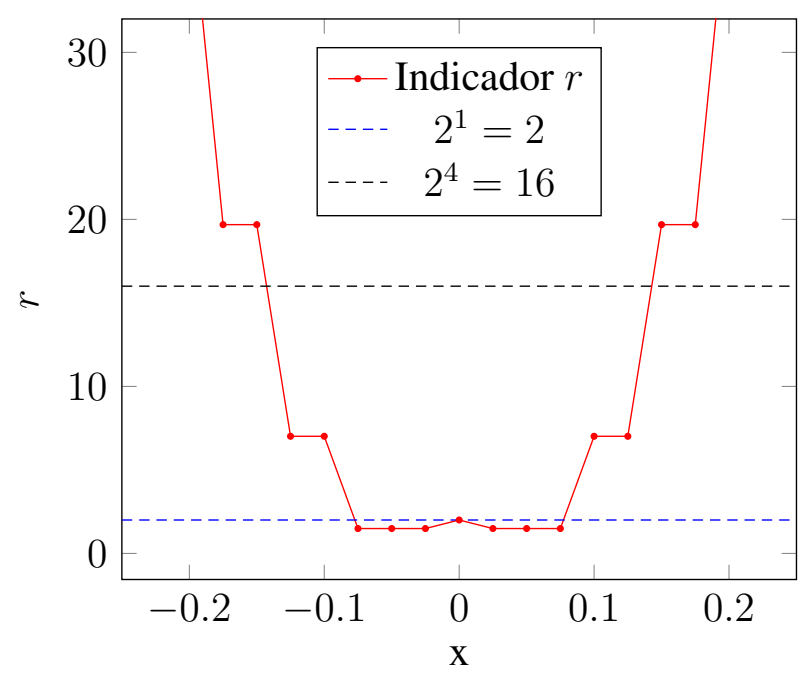

(c) Indicador - Ampliação - 81 pontos

Figura 3.4: Relação $2^{n}$ - Função Módulo

Nota-se que em $x=0$ existe uma descontinuidade na primeira derivada da função Módulo, como pode ser observado na Figura 3.4(b). Os valores obtidos para o indicador $r$ nas regiões suaves são maiores ou próximos a 16 , sendo que na descontinuidade o valor obtido para esse parâmetro é $2^{1}=2$, evidenciando assim a relação $2^{n}$ e indicando que a descontinuidade dessa função se encontra de fato na primeira derivada.

Além disso, observa-se com mais clareza na Figura 3.4(c) que os valores obtidos na descontinuidade são próximos a 2. 


\subsubsection{Segunda Derivada Descontínua}

A Figura 3.5 mostra a função (3.33) com segunda derivada descontínua em $x=0$, apresentando assim valores diferentes para o indicador $r$ nessa região.

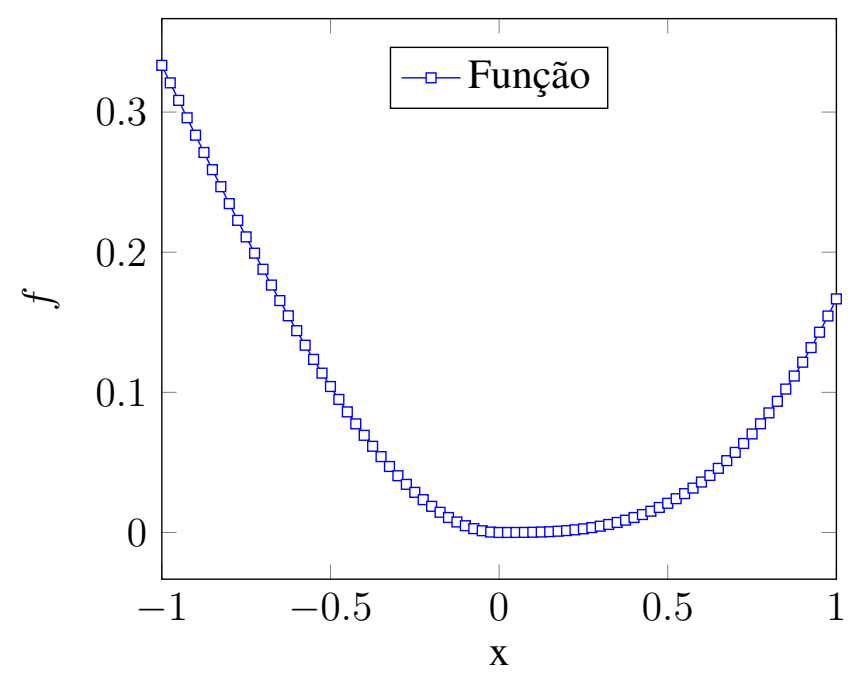

(a) Função - 81 pontos

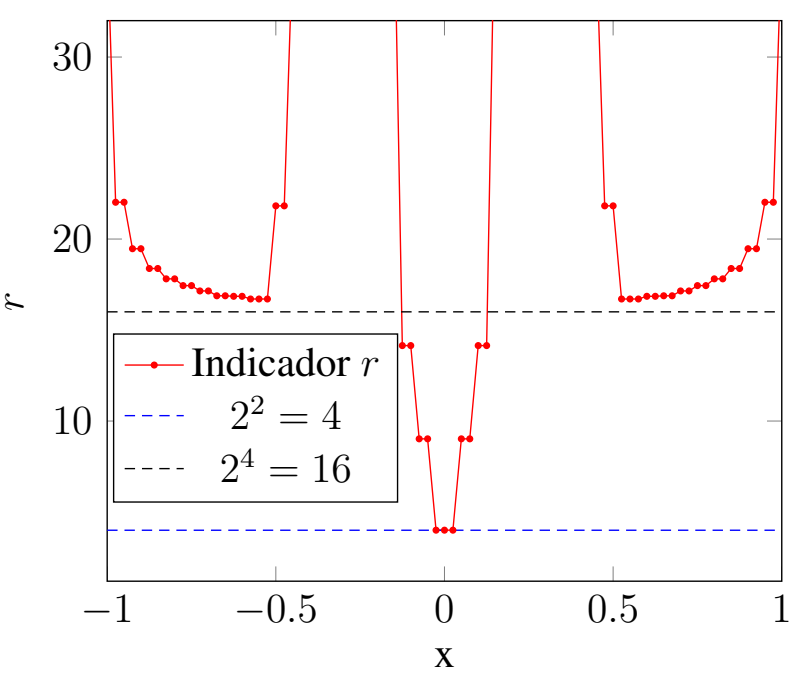

(b) Indicador - 81 pontos

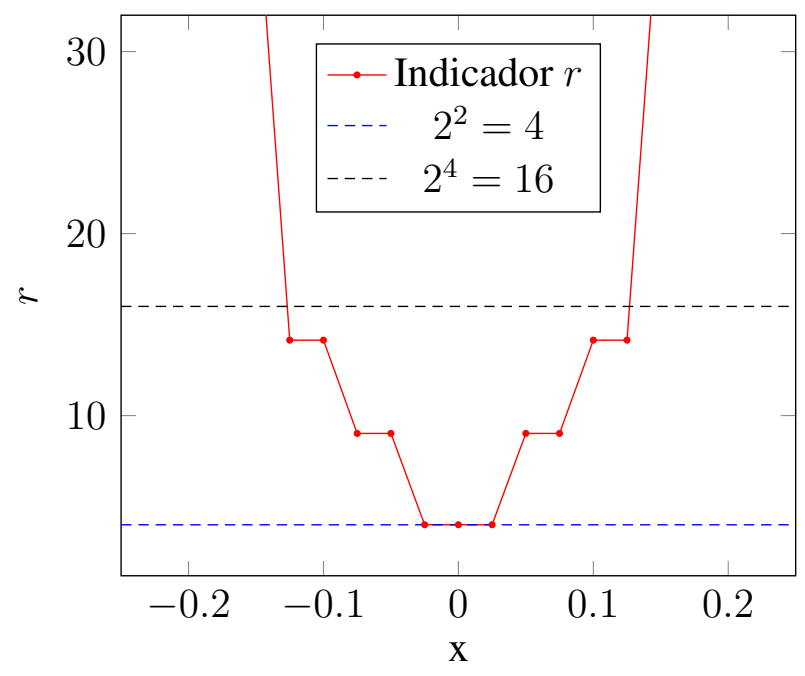

(c) Indicador - Ampliação - 81 pontos

Figura 3.5: Relação $2^{n}$ - Segunda Derivada Descontínua

Observa-se que as regiões suaves apresentam valores maiores ou próximos a 16, sendo que em $x=0$ esses valores mudam para muito próximos de $2^{2}=4$, resultando na relação $2^{n}$ apresentada na equação (3.28).

\subsubsection{Terceira Derivada Descontínua}

A Figura 3.6 apresenta a função (3.34) que apresenta uma descontinuidade em $x=0$ na terceira derivada, sendo possível observar diferentes valores para o indicador $r$. 


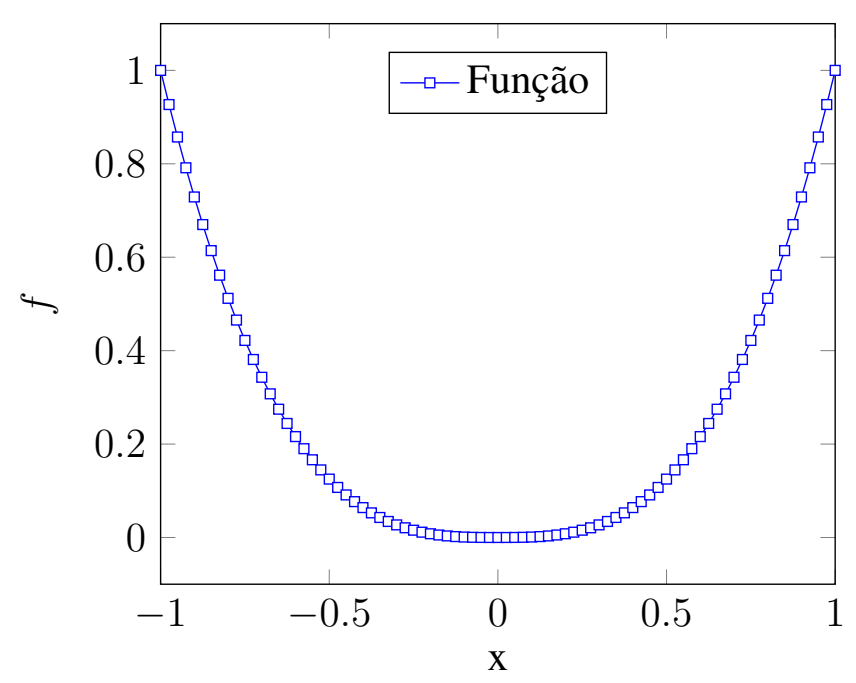

(a) Função - 81 pontos

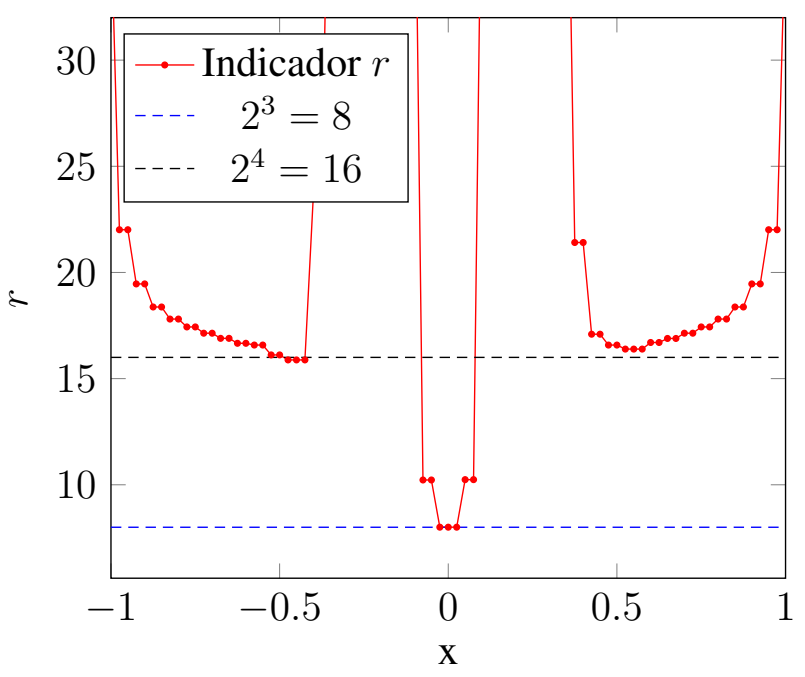

(b) Indicador - 81 pontos

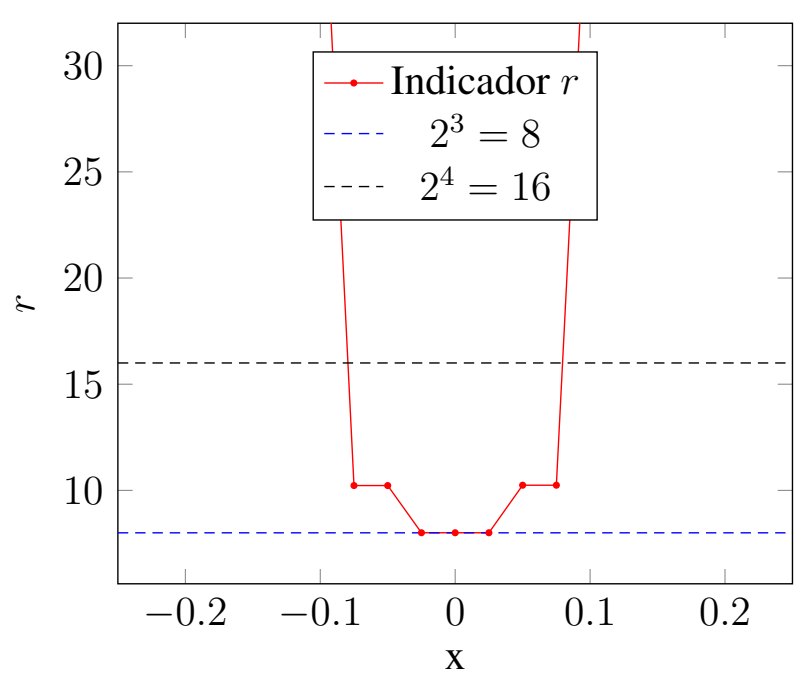

(c) Indicador - Ampliação - 81 pontos

Figura 3.6: Relação $2^{n}$ - Terceira Derivada Descontínua 
Como é possível observar nas Figuras 3.6(b) e 3.6(c), os valores do indicador $r$ nas regiões suaves são diferentes da região descontínua, sendo que para a primeira o mesmo apresenta valores maiores ou próximos a 16 , enquanto que para o último os valores obtidos são muito próximos a $2^{3}=8$, mostarando assim a relação $2^{n}$ apresentada na equação (3.28).

\subsubsection{Frequência Alta}

A Figura 3.7 apresenta o indicador $r$ para a função (3.35) com derivadas contínuas, sendo que esta depende do número de pontos utilizados na discretização, como pode ser observado na equação (3.35). O número de pontos na discretização considerado foi 81.

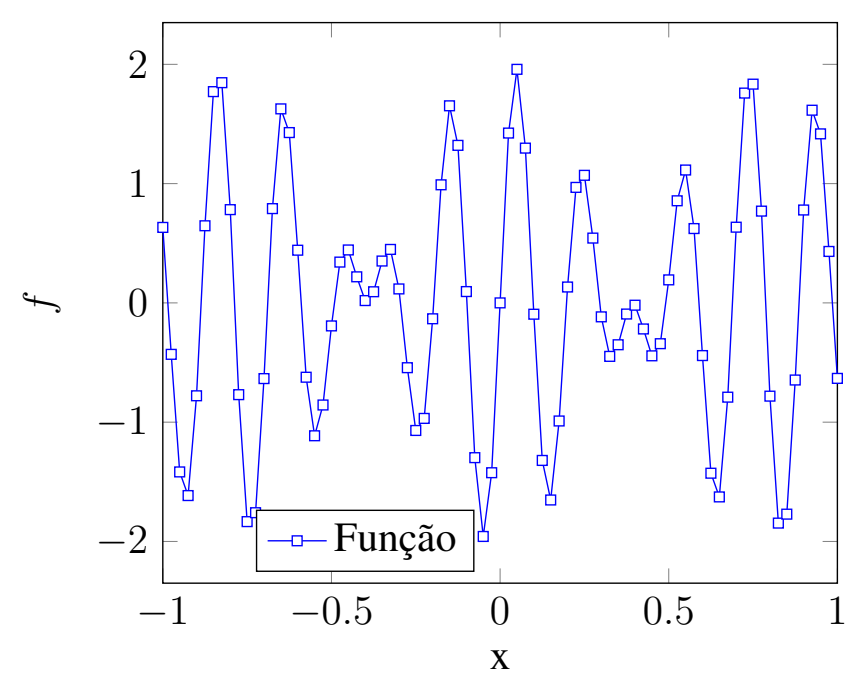

(a) Função - 81 pontos

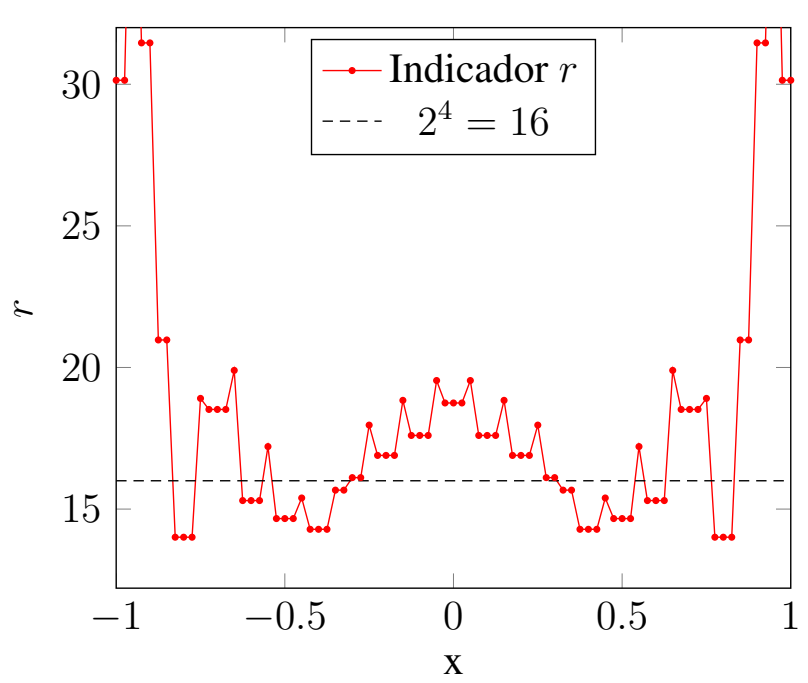

(b) Indicador - 81 pontos

Figura 3.7: Relação $2^{n}$ - Alta Frequência

Nota-se que os valores obtidos para o indicador $r$ presente no detector desenvolvido são maiores ou próximos a 16, indicando assim que toda a função e suas derivadas são suaves.

\subsection{Observações}

Através de diversas funções e gráficos que permitem visualizar os valores obtidos para o indicador $r$, foi possível observar e avaliar a relação $2^{n}$ apresentada nas equações (3.25) e (3.28). Nota-se que, quando a região é suave, o indicador apresenta valores maiores ou próximos a 16; já quando a descontinuidade está na própria função considerada, o valor observado nos gráficos é $2^{0}=1$ na região onde se encontra a descontinuidade, assim como foi discutido teoricamente. O mesmo ocorre quando a função apresenta descontinuidade na primeira, segunda ou terceira derivada, alterando apenas o valor da relação $2^{n}$. 
A grande importância da relação $2^{n}$ é, através do indicador $r$, a possibilidade de determinar com o detector desenvolvido em qual derivada se encontra a descontinuidade, ou podendo a mesma estar na própria função.

Com a relação $2^{n}$ definida, é possível avaliar o desempenho do novo detector para diferentes tipos de funções unidimenionais (1D) e bidimensionais (2D), verificando assim se as regiões com função suave ou descontínua são determinadas corretamente. Além disso, após a verificação do detector, o método híbrido desenvolvido será aplicado para resolver as equações de Euler 1D e 2D. 
Neste capítulo são apresentados os resultados obtidos com o novo detector desenvolvido para diferentes funções unidimensionais e bidimensionais, realizando assim uma comparação com as diversas funções detectoras já existentes. A comparação com Zhou [36], Pirozzoli [21], Adams [2], Yee [33], Hill [11] e Kim [14] permite avaliar o desempenho dessa nova função detectora, ou seja, se as regiões com função suave ou descontínuas são determinadas corretamente. Além disso, é possível avaliar as principais vantagens e desvantagens que cada método apresenta.

As equações de Euler unidimensionais (1D) e bidimensionais (2D) também são resolvidas considerando diferentes condições iniciais. Para obter os resultados para essas equações numericamente, é utilizado o novo detector de descontinuidades desenvolvido juntamente com os métodos WENO [13] e diferenças centrais, construindo-se então um método numérico híbrido. A fim de comparar e avaliar a solução numérica obtida, as equações de Euler são também resolvidas utilizando-se apenas o método WENO, detalhado no apêndice A. Além disso, os métodos numéricos híbrido e WENO são utilizados para calcular as derivadas de funções simples, obtendo assim o erro numérico de cada método.

Dessa forma, tanto para o caso 1D como para o 2D, com a intenção de evitar o cálculo da derivada pelo método central utilizando pontos de uma região com função descontínua, constróise uma vizinhança em torno da descontinuidade. Sejam $N_{c}$ e $N_{w}$ as ordens dos métodos central e WENO, respectivamente. O número de pontos necessários para construir a vizinhança para o método central é $N_{c} / 2$ e para o método WENO é $\left(N_{w}+1\right) / 2$, sendo $k=N_{c} / 2$ o número de pontos na vizinhança à direita e à esquerda que também serão considerados como descontínuos. Assim, tem-se $k=3$, já que o método de diferenças centrais é de ordem 6 e o método WENO de ordem 5 . 


\subsection{Detector Desenvolvido - 1D}

Para mostrar os resultados obtidos com o novo detector de descontinuidades desenvolvido, diferentes funções unidimensionais foram consideradas, permitindo assim realizar comparações com outras funções detectoras já desenvolvidas e estudadas durante a revisão bibliográfica.

Os pesos $(\sigma)$ indicam se uma determinada região é considerada com função suave ou descontínua, resultando assim em um vetor com dimensão $N_{x}$, que representa o número de pontos utilizados na discretização.

As funções unidimensionais consideradas foram:

- Função Cosseno:

$$
f(x)=\cos (\pi x), \quad x \in[-1,1]
$$

- Função Mesa:

$$
f(x)=\left\{\begin{array}{ll}
1, & \text { se }-\frac{1}{5} \leq x \leq \frac{1}{5} \\
0, & \text { caso contrário }
\end{array}, \quad x \in[-1,1]\right.
$$

- Função Seno Descontínuo:

$$
f(x)=\left\{\begin{array}{ll}
\operatorname{sen}(3 \pi x)+2.5, & \text { se }-\frac{1}{5} \leq x \leq \frac{1}{5} \\
\operatorname{sen}(3 \pi x), & \text { caso contrário }
\end{array}, \quad x \in[-1,1]\right.
$$

- Função Módulo:

$$
f(x)=\left\{\begin{array}{ll}
x, & \text { se } x>0 \\
-x, & \text { se } x<0
\end{array}, \quad x \in[-1,1]\right.
$$

- Função Frequência Alta:

$$
f(x)=\operatorname{sen}\left(\frac{\left(N_{x}-1\right) \pi x}{7}\right)+\operatorname{sen}\left(\frac{\left(N_{x}-1\right) \pi x}{9}\right), \quad x \in[-1,1] .
$$

Algumas dessas funções são contínuas com derivadas contínuas como os casos da Cosseno e Frequência Alta, outras apresentam descontinuidades na própria função como Mesa e Seno Descontínuo. Foi considerada também a função Módulo que apresenta descontinuidade na primeira derivada.

Com o estudo do valor limiar e da relação $2^{n}$, é possível determinar o valor de $\hat{r}$ de modo a indicar corretamente as regiões que apresentam função suave ou descontínua. $\mathrm{O}$ valor escolhido para esse parâmetro foi 2.82, com a intenção de determinar a região como sendo descontínua até a primeira derivada da função $f(x)$. 
Teoricamente, para determinar até a primeira derivada, o valor para $\hat{r}$ deveria ser $2^{1}=2$. Porém, como as derivadas e consequentemente a equação (2.18) são calculadas numericamente, é necessário considerar um erro numérico no cálculo dessas variáveis e indicação das regiões. Assim, a ideia foi escolher o valor limiar $\hat{r}=2 \sqrt{2} \approx 2.82$ de forma que se o valor de $r_{i}$ for menor a $\hat{r}$ então a região apresenta função descontínua. Caso contrário, a região é determinada como suave.

\subsubsection{Cosseno}

As Figuras 4.1 e 4.2 mostram os resultados obtidos para os diferentes detectores, indicando os valores calculados para os pesos $\sigma$.

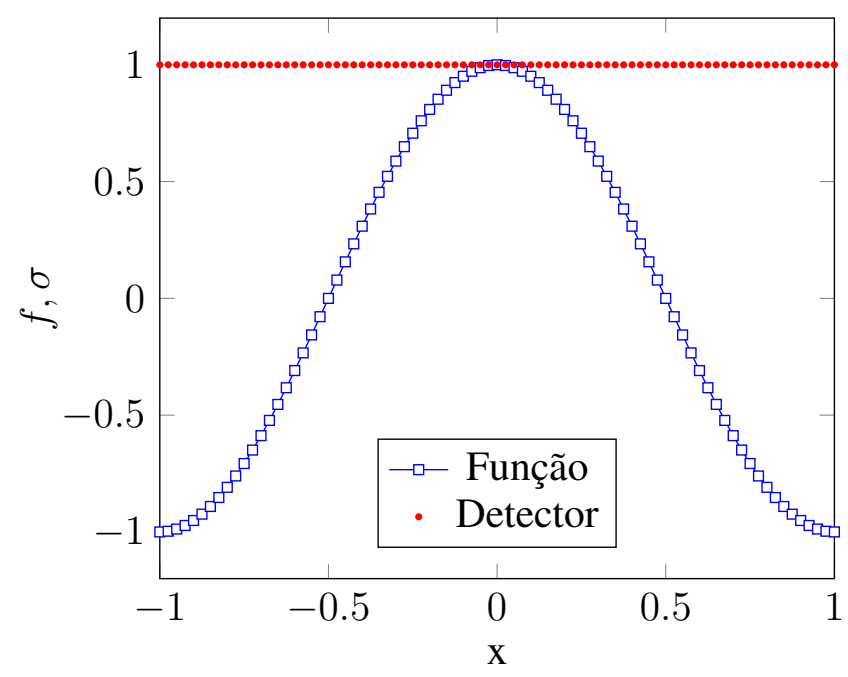

(a) Adams - 81 pontos

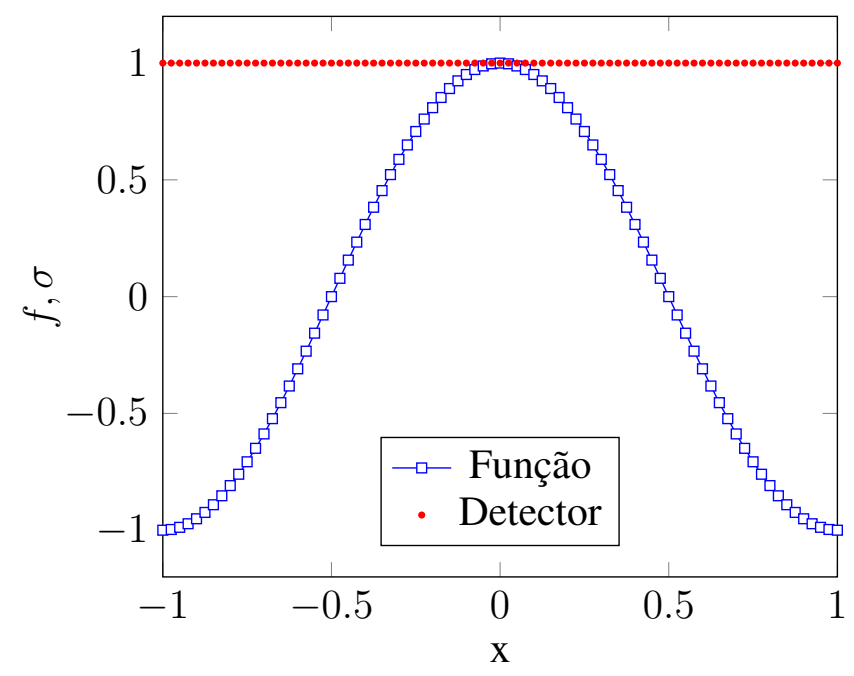

(c) Kim - 81 pontos

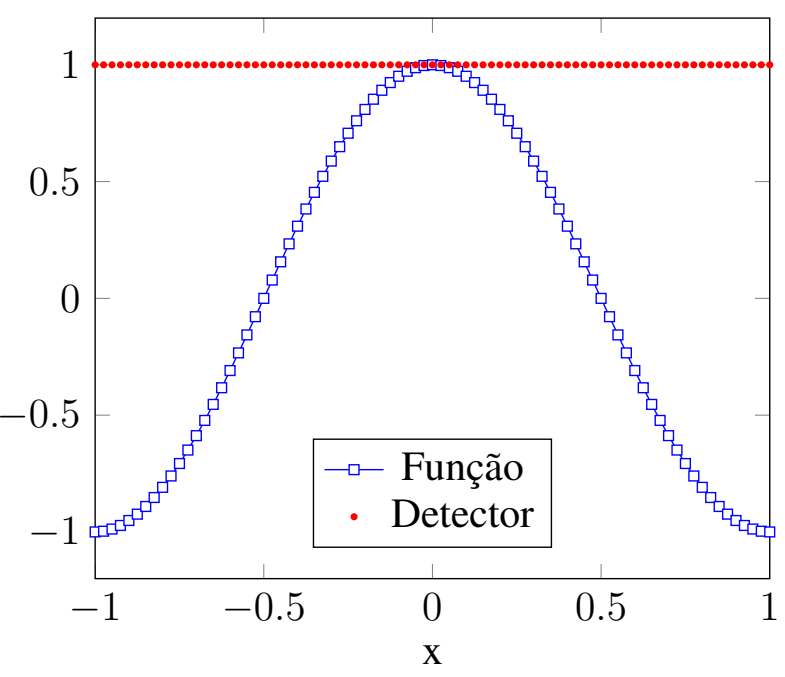

(b) Yee - 81 pontos

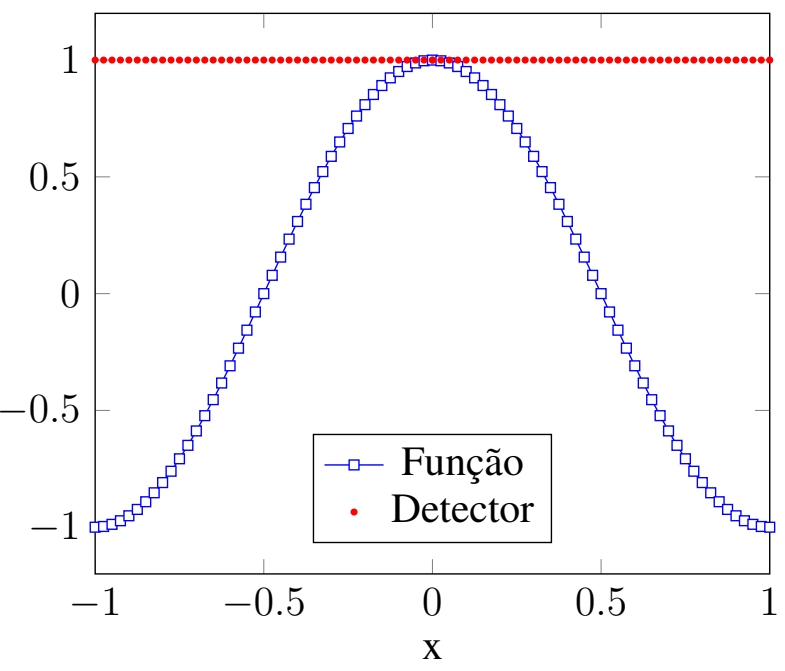

(d) Hill - 81 pontos

Figura 4.1: Detectores 1D - Função Cosseno

Como a função Cosseno (4.1) e suas derivadas são contínuas, espera-se que todos os pesos $\sigma$ calculados tenham valor 1 , indicando assim que a função é suave em todas as regiões. Isso de fato 


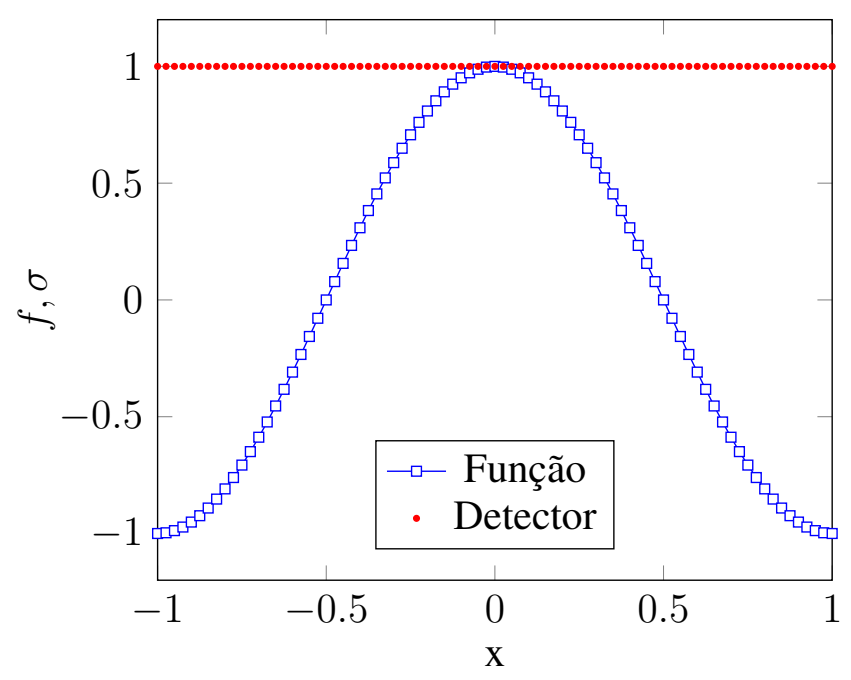

(a) Pirozzoli - 81 pontos

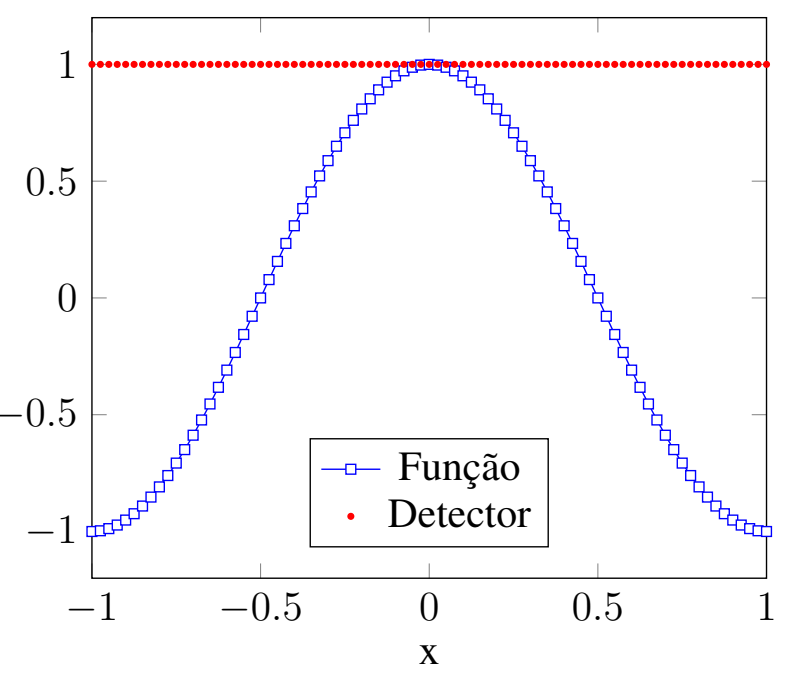

(b) Zhou - 81 pontos

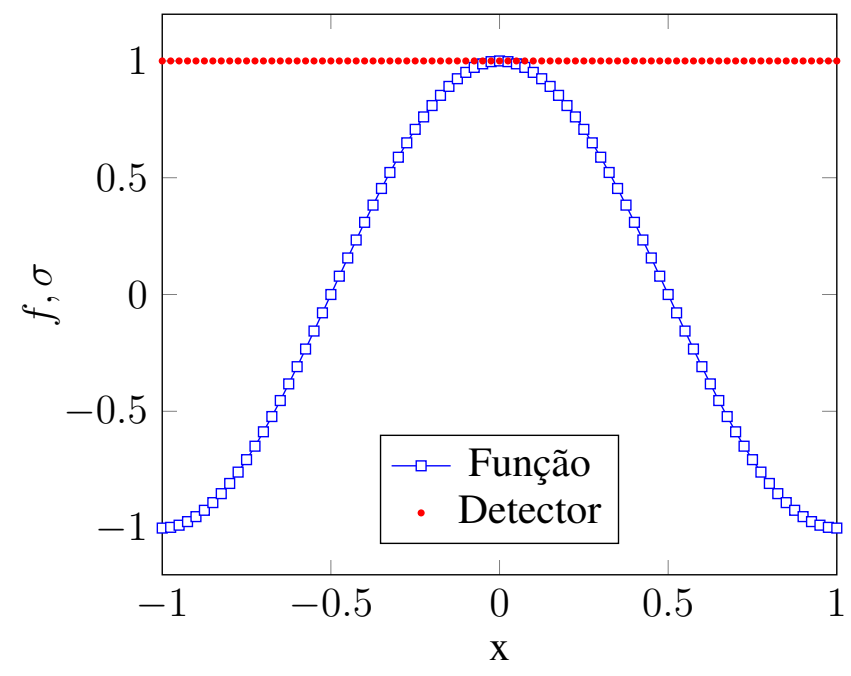

(c) Novo detector - 81 pontos

Figura 4.2: Detectores 1D - Função Cosseno - continuação 
ocorre para todos os detectores estudados, inclusive para a nova função detectora desenvolvida com 81 pontos na discretização.

\subsubsection{Mesa}

As Figuras 4.3 e 4.4 mostram os resultados para a função Mesa (4.2) com 81 pontos na discretização.

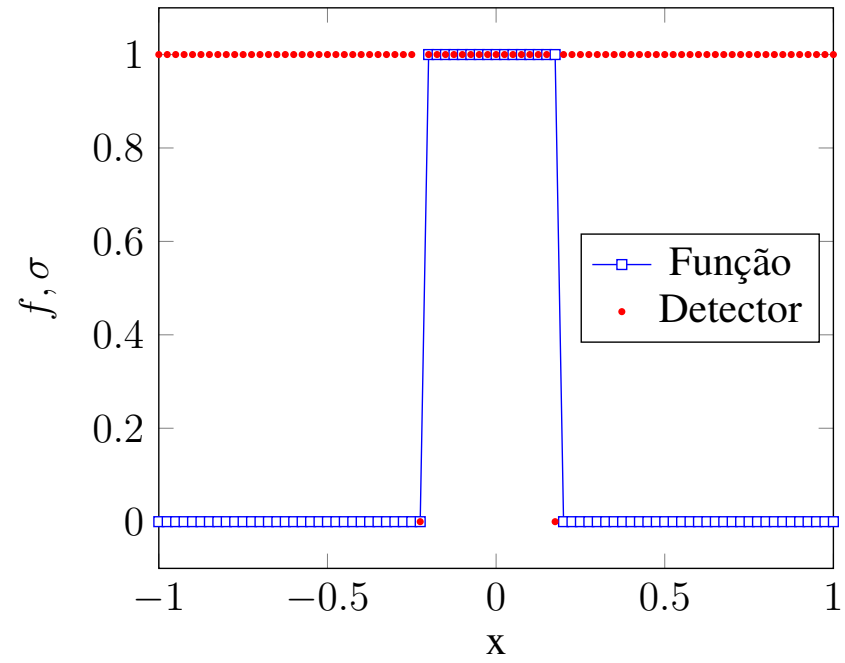

(a) Adams - 81 pontos

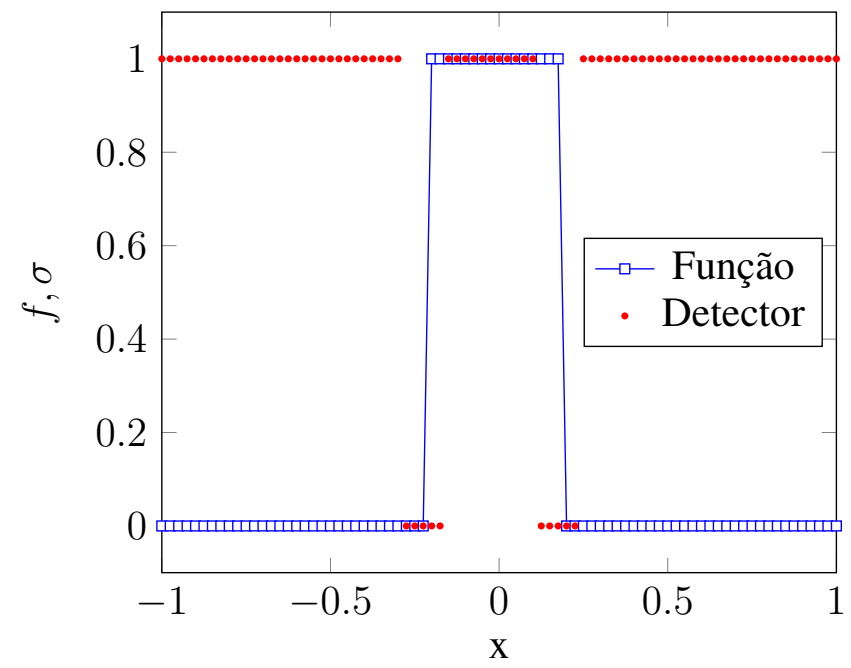

(c) Kim - 81 pontos

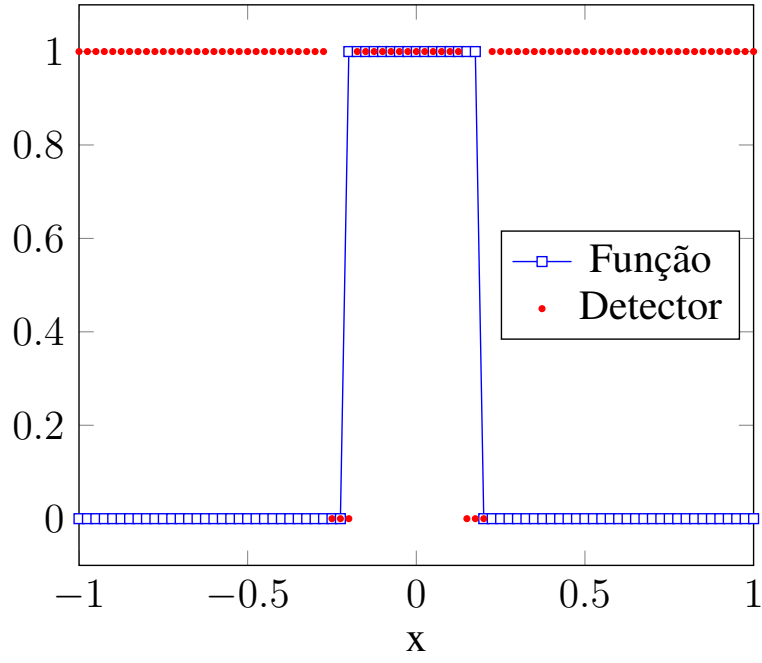

(b) Yee - 81 pontos

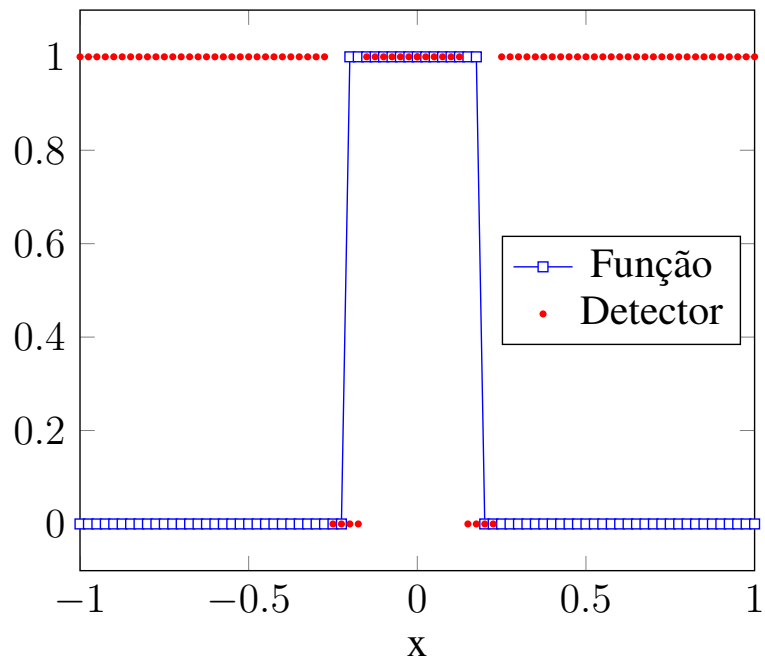

(d) Hill - 81 pontos

Figura 4.3: Detectores 1D - Função Mesa

É possível observar pelos gráficos que a função Mesa apresenta uma descontinuidade nos pontos $x=-1 / 5$ e $x=1 / 5$, sendo que os detectores de descontinuidades devem considerar peso $\sigma=0$ nas regiões ao redor desses pontos. Isso de fato ocorre para todas as funções detectoras estudadas, inclusive para o novo detector desenvolvido, como pode ser visto na Figura 4.4(c). 


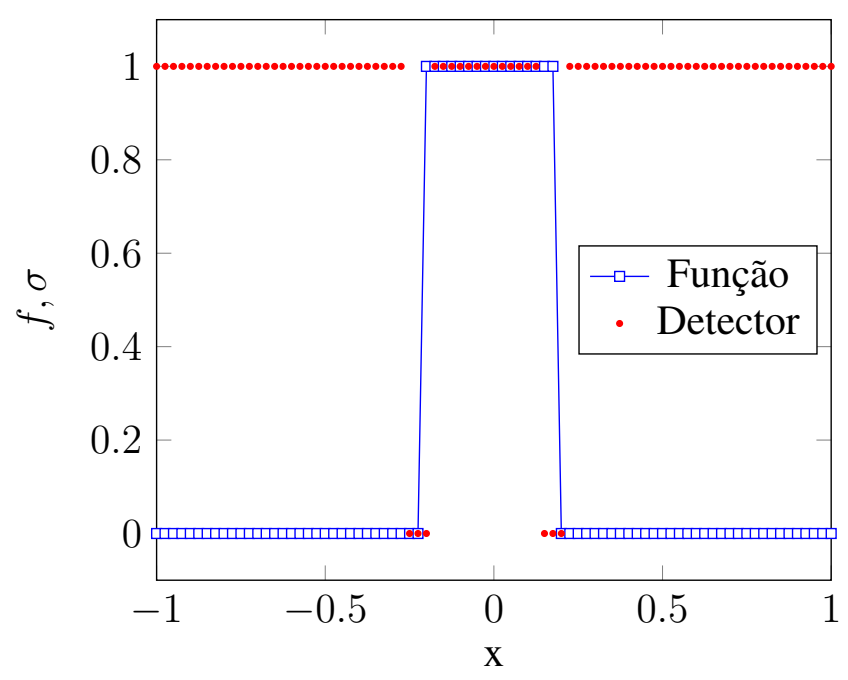

(a) Pirozzoli - 81 pontos

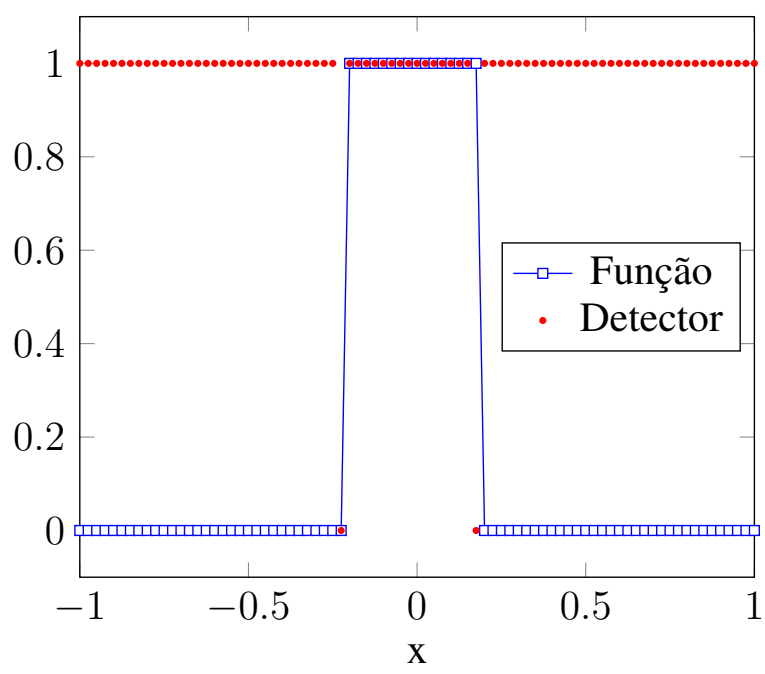

(b) Zhou - 81 pontos

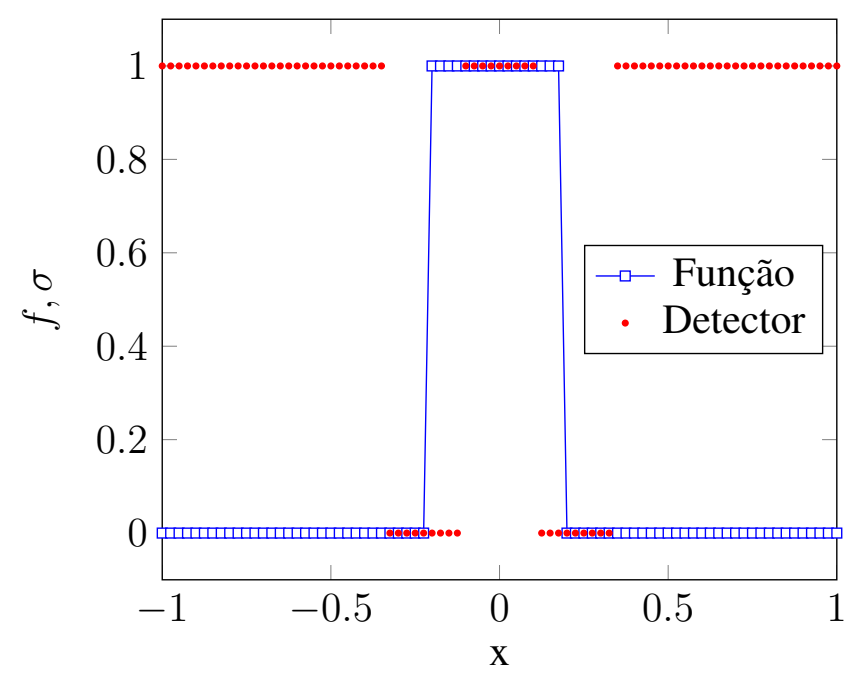

(c) Novo detector - 81 pontos

Figura 4.4: Detectores 1D - Função Mesa - continuação 


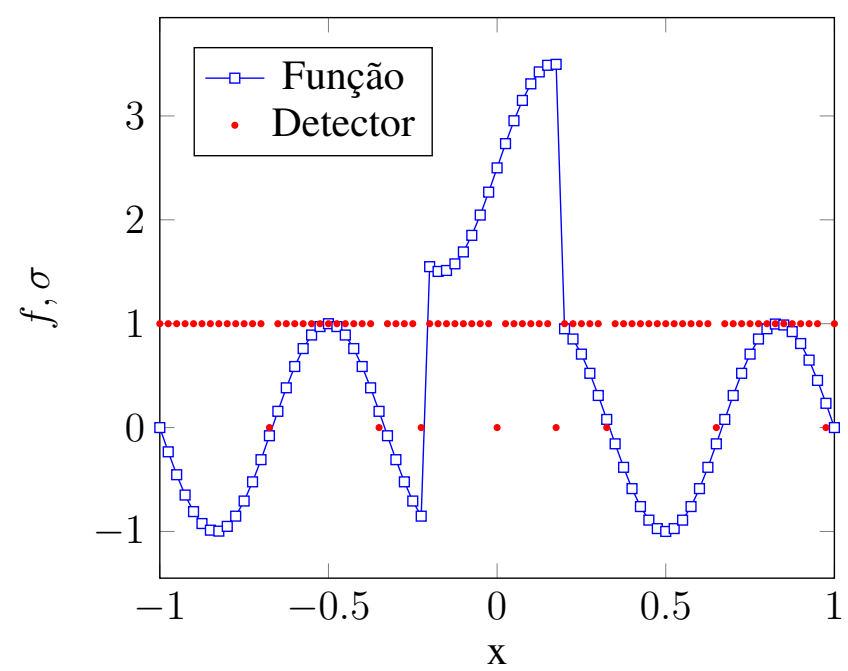

(a) Adams - 81 pontos

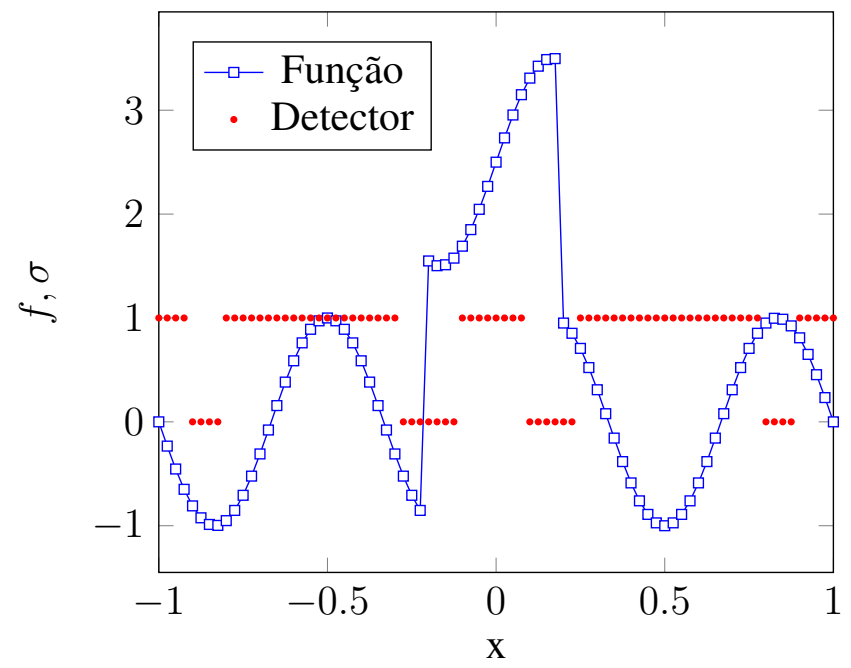

(c) Kim - 81 pontos

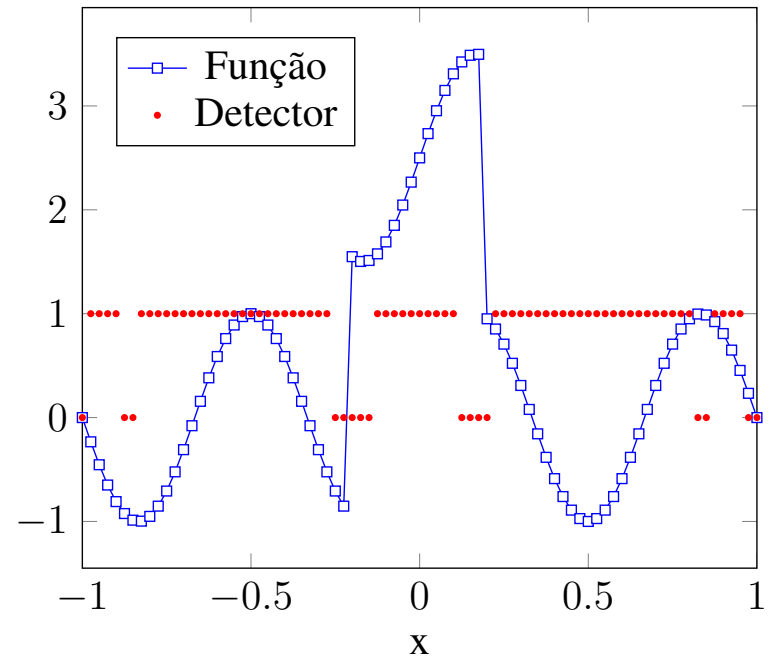

(b) Yee - 81 pontos

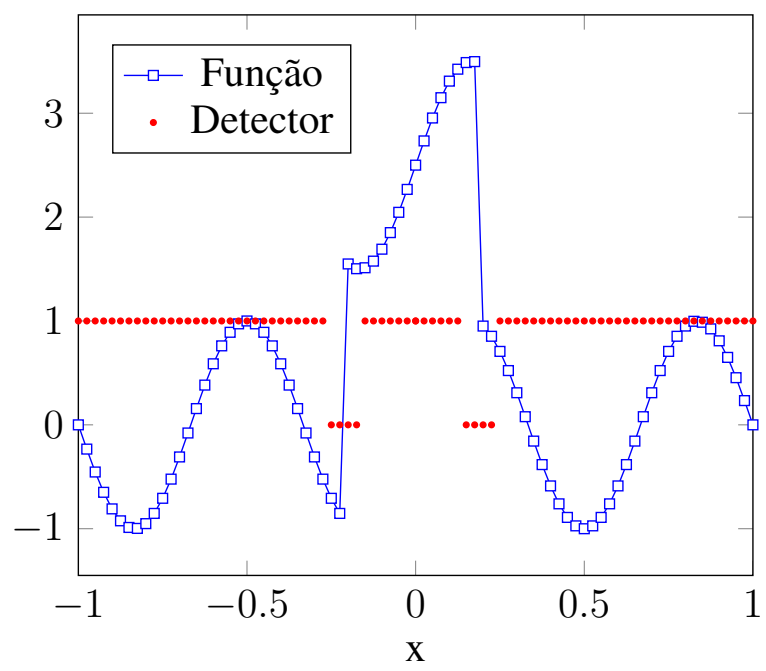

(d) Hill - 81 pontos

Figura 4.5: Detectores 1D - Função Seno Descontínuo 


\subsubsection{Seno Descontínuo}

As Figuras 4.5 e 4.6 mostram os resultados obtidos para a função Seno Descontínuo (4.3), que assim como a função Mesa apresenta descontinuidades em $x=-1 / 5$ e $x=1 / 5$.

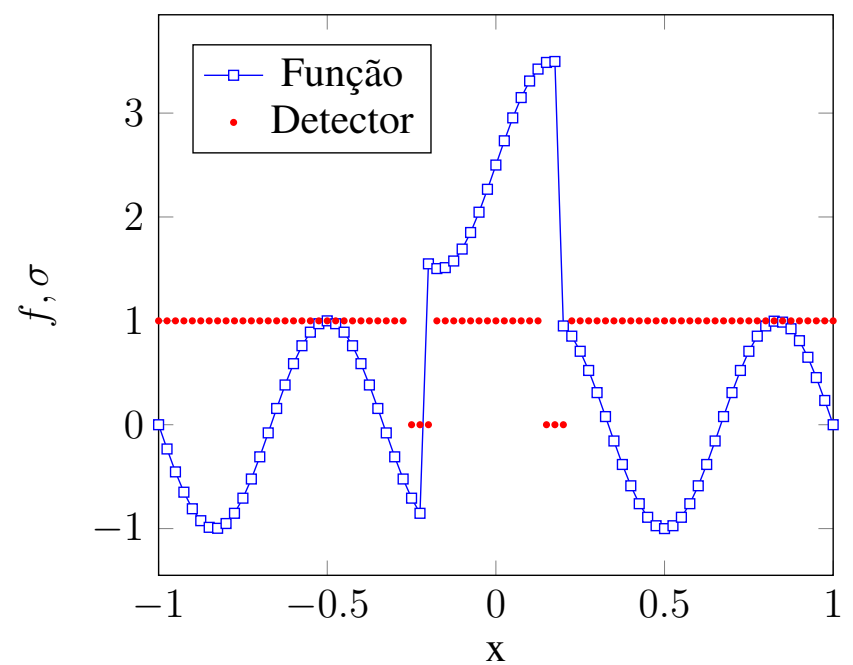

(a) Pirozzoli - 81 pontos

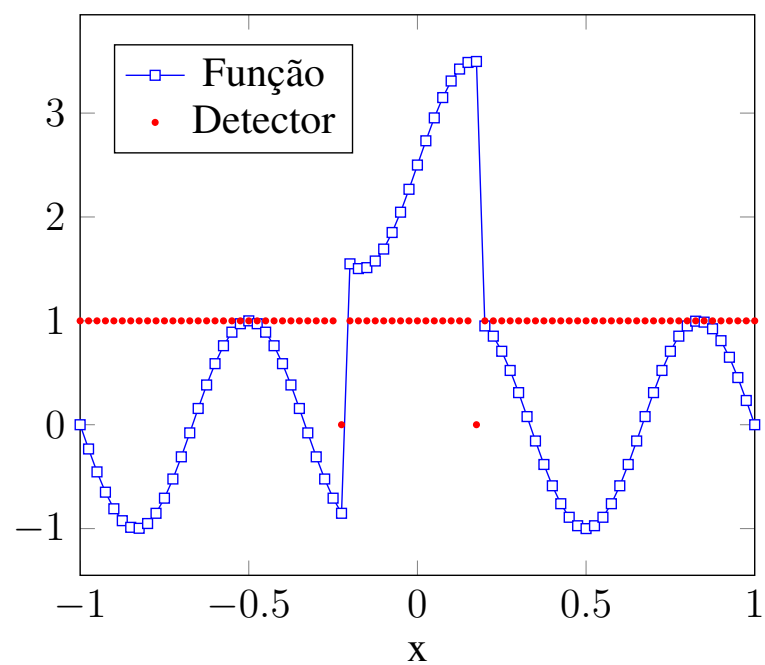

(b) Zhou - 81 pontos

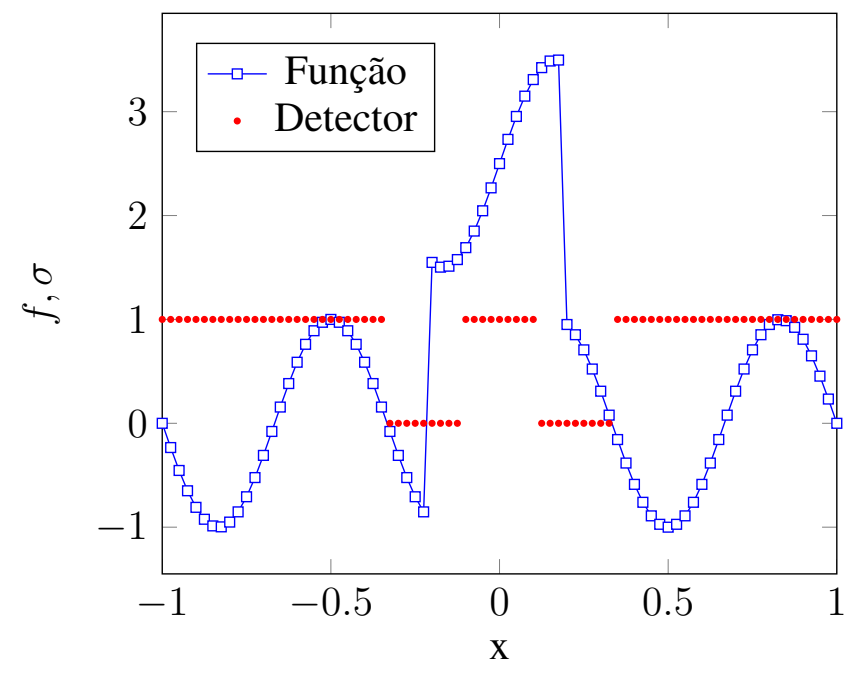

(c) Novo detector - 81 pontos

Figura 4.6: Detectores 1D - Função Seno Descontínuo - continuação

Nota-se que, para essa função, os detectores desenvolvidos por Adams, Yee e Kim apresentam problemas em determinar quais regiões são suaves e quais são descontínuas, sendo que pelas Figuras 4.5(a), 4.5(b) e 4.5(c) é possível ver que as regiões que são de fato suaves foram consideradas como descontínuas. O mesmo não ocorre para os detectores Hill, Pirozzoli, Zhou e o novo detector, determinando as regiões corretamente. 


\subsubsection{Módulo}

As figuras 4.7 e 4.8 mostram os resultados dos diversos detectores estudados para a função Módulo (4.4). Observa-se que essa função apresenta descontinuidade em $x=0$ na primeira derivada.

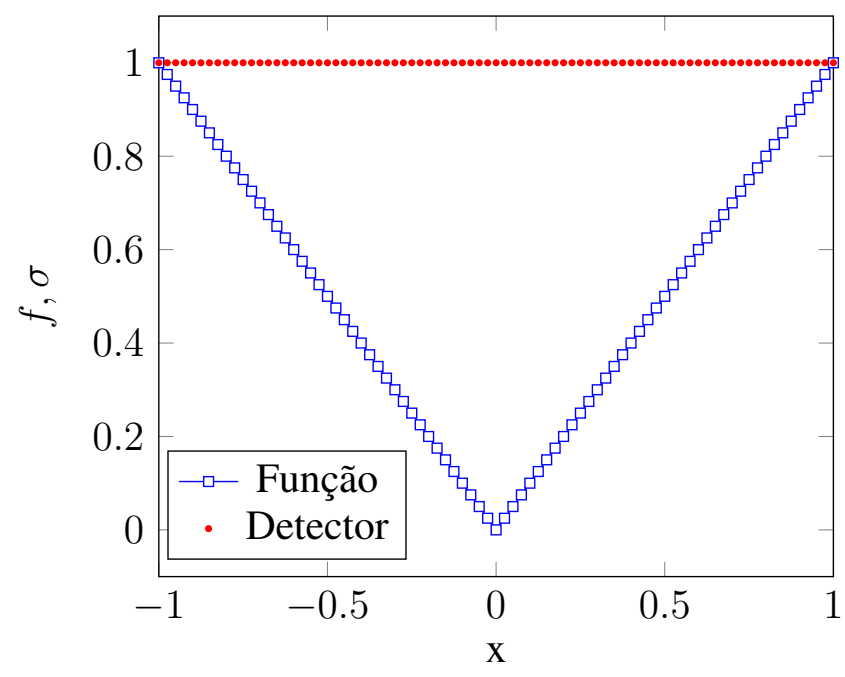

(a) Adams - 81 pontos

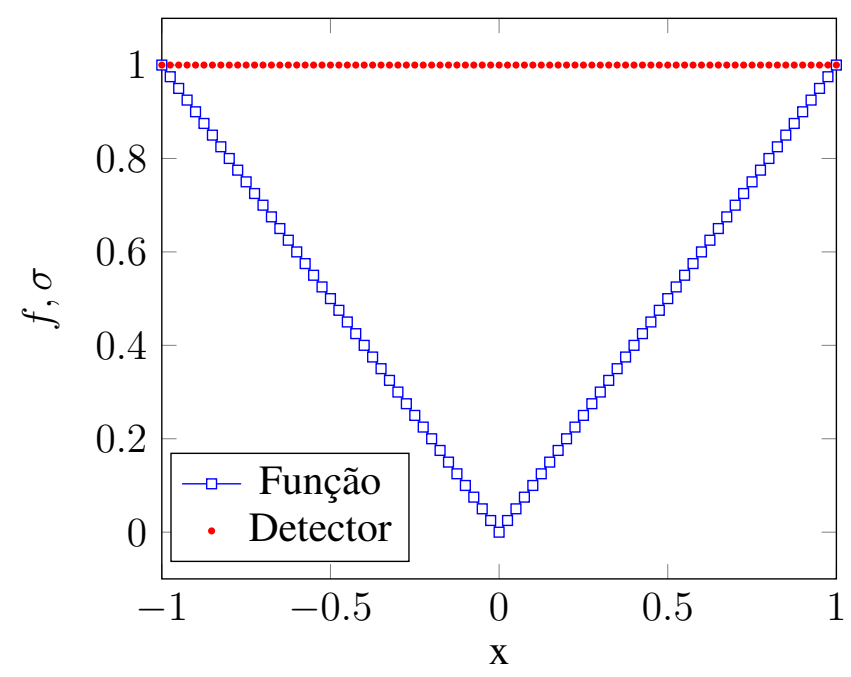

(c) Kim - 81 pontos

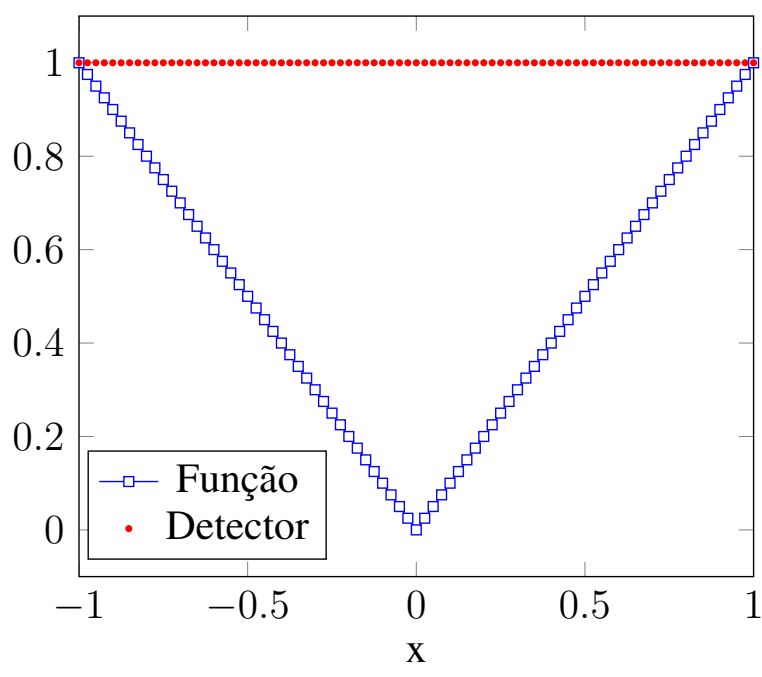

(b) Yee - 81 pontos

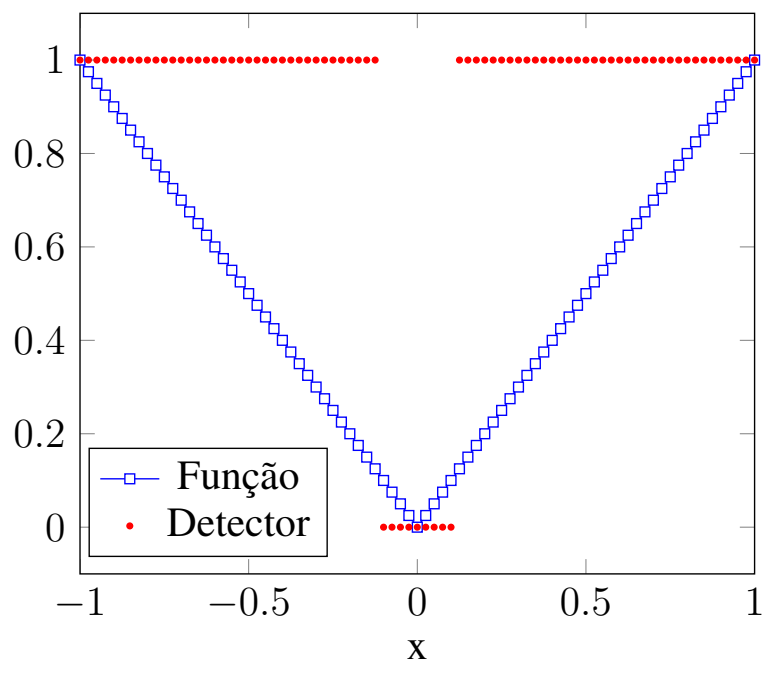

(d) Hill - 81 pontos

Figura 4.7: Detectores 1D - Função Módulo

Apenas o detector Hill e o novo determinaram a região de $x=0$ como sendo descontínua, já que estes avaliam derivadas da função para definir se uma região pode ser considerada suave ou descontínua, como pode ser observado pelas Figuras 4.7(d) e 4.8(c). O mesmo não ocorre para os demais detectores, que definem toda a função como sendo suave.

Para o novo detector desenvolvido, essa região é considerada como sendo descontínua, pois avalia a derivada da função e o valor limiar é $\hat{r}=2.82$, permitindo que regiões cuja derivada da função é descontínua sejam avaliadas com pesos $\sigma=0$. 


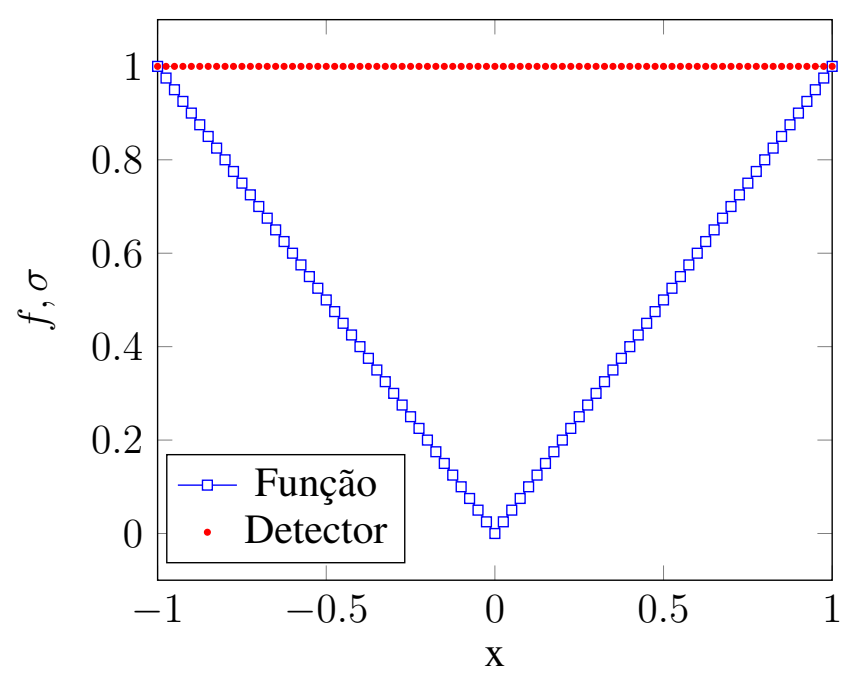

(a) Pirozzoli - 81 pontos

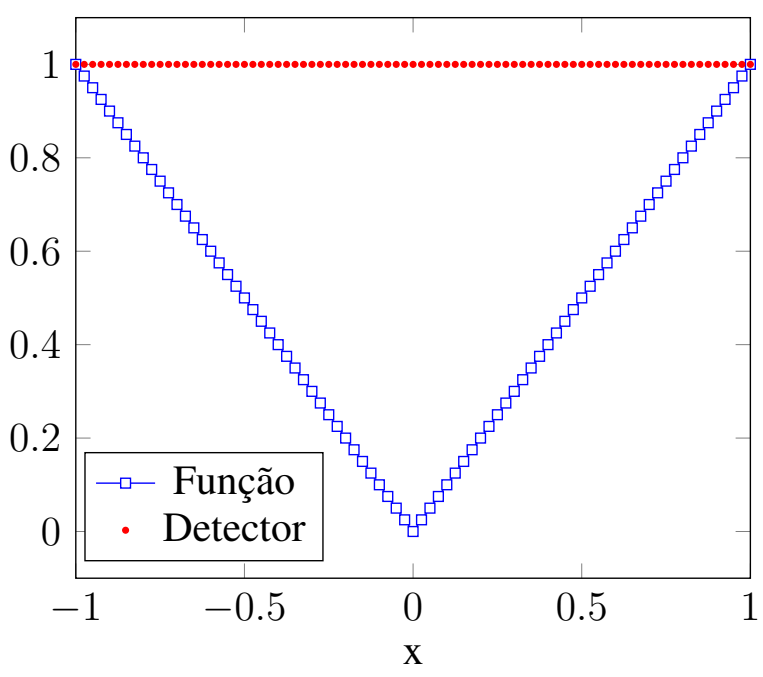

(b) Zhou - 81 pontos

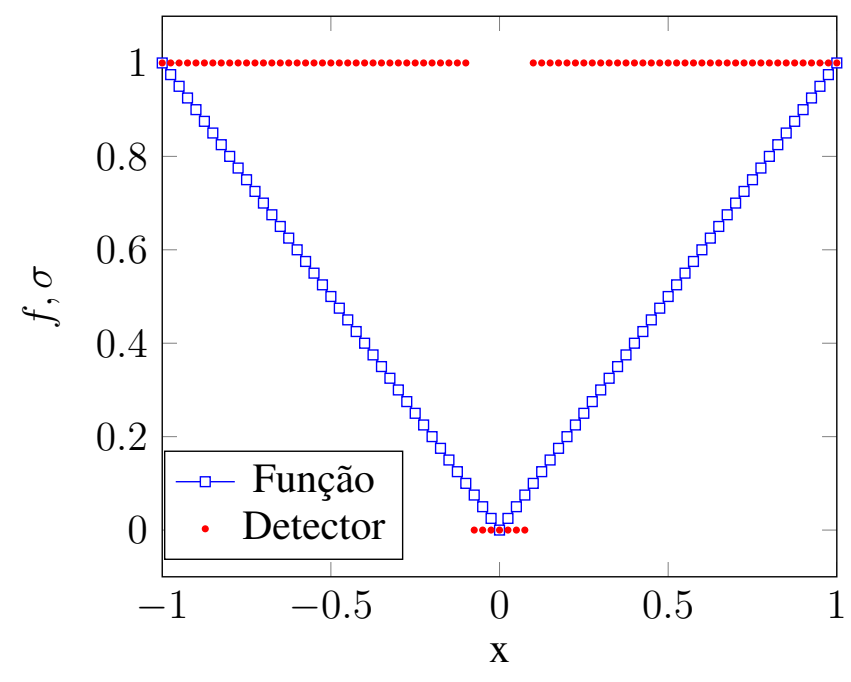

(c) Novo detector - 81 pontos

Figura 4.8: Detectores 1D - Função Módulo - continuação 


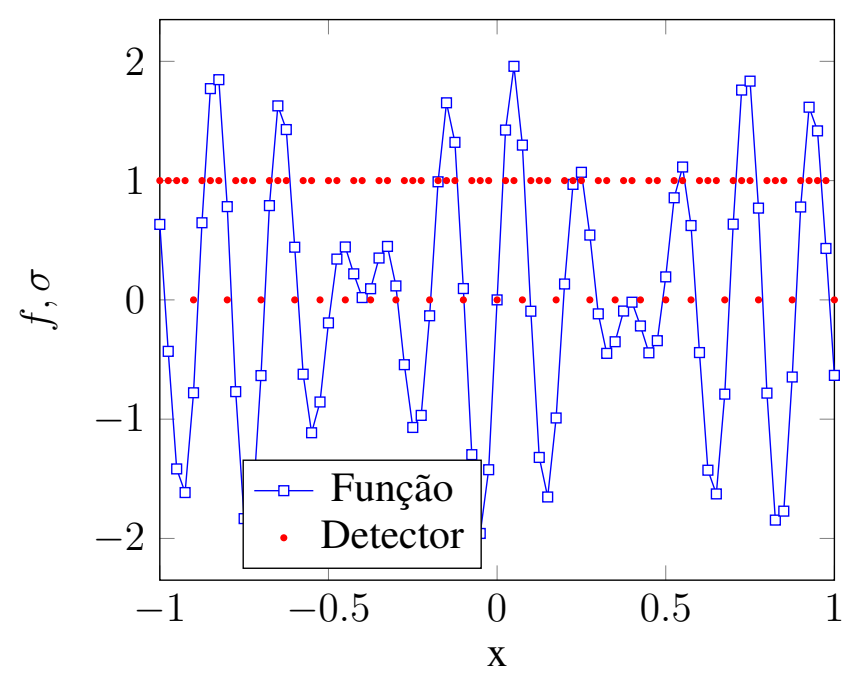

(a) Adams - 81 pontos

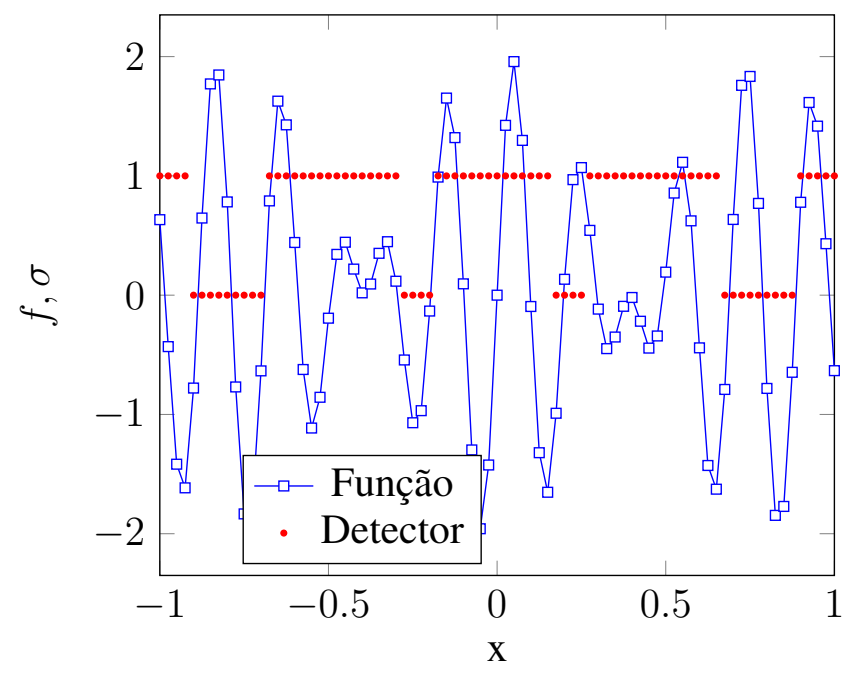

(c) Kim - 81 pontos

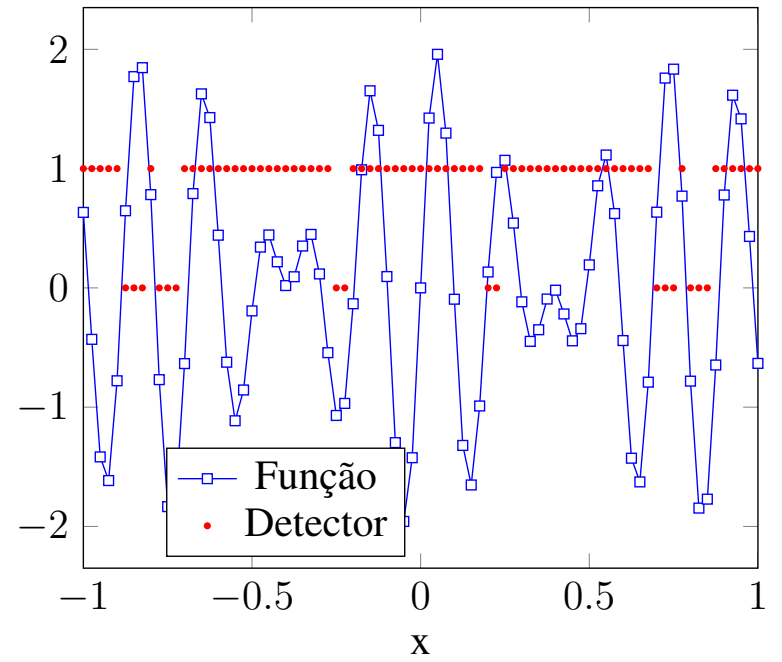

(b) Yee - 81 pontos

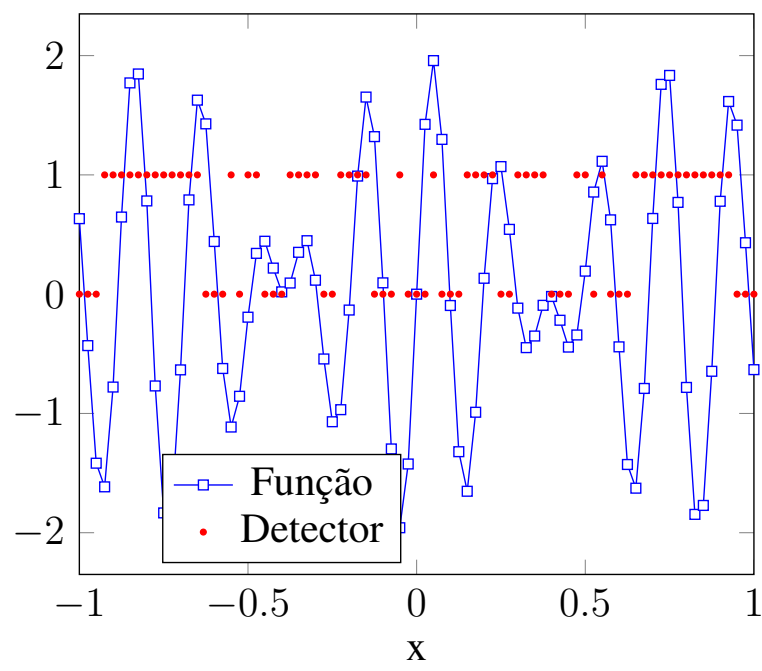

(d) Hill - 81 pontos

Figura 4.9: Detectores 1D - Função Frequência Alta 


\subsubsection{Frequência Alta}

As Figuras 4.9 e 4.10 mostram os resultados dos detectores para a função Frequência Alta (4.5), que é contínua e possui derivadas contínuas.

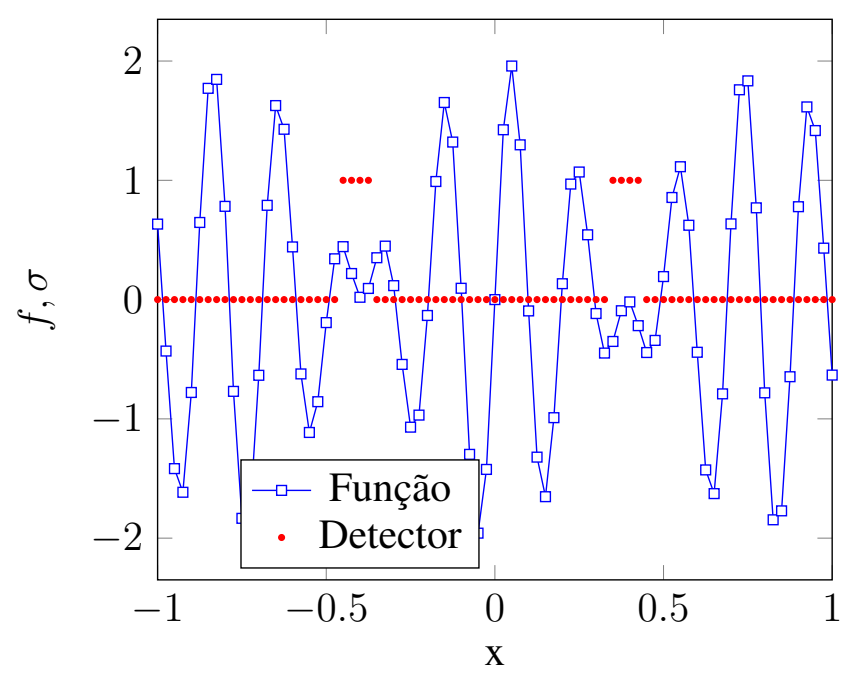

(a) Pirozzoli - 81 pontos

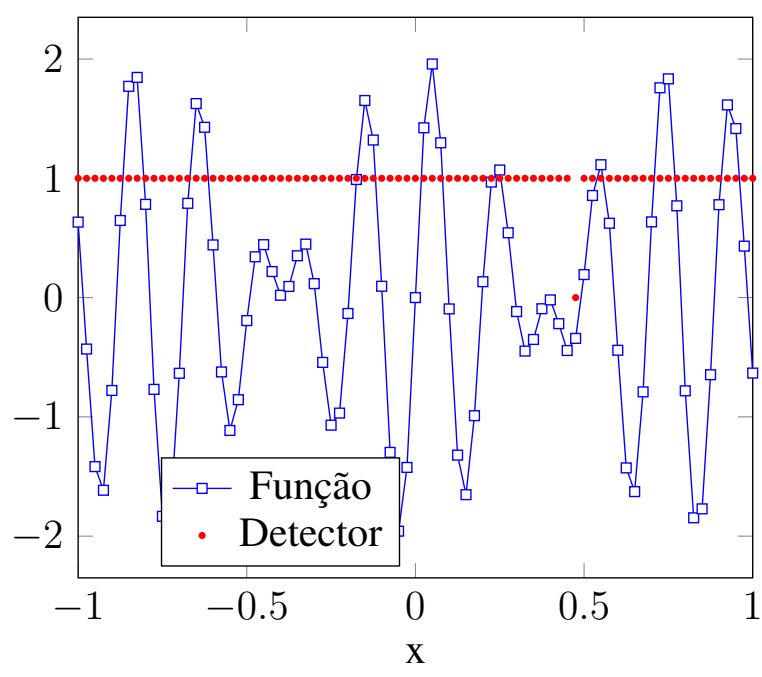

(b) Zhou - 81 pontos

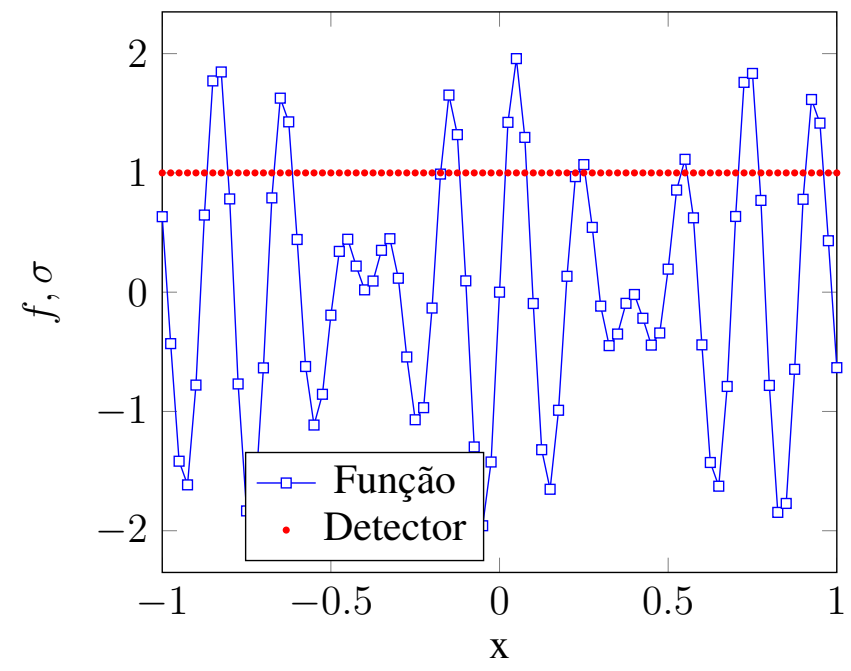

(c) Novo detector -81 pontos

Figura 4.10: Detectores 1D - Função Frequência Alta - continuação

Observa-se que, como a função considerada é contínua e apresenta derivadas contínuas, todas as regiões devem ser consideradas como sendo suaves pelos detectores de descontinuidades. Isso ocorre apenas através da nova função detectora desenvolvida, como pode ser visto na Figura 4.10(c), sendo que todos os outros detectores estudados apresentam problemas em determinar se a região de fato é suave. O detector de Zhou, em particular, apresenta apenas um ponto de descontinuidade.

A função Frequência Alta possui uma característica interessante com grandes variações, o que permite avaliar de uma forma mais rígida o desempenho de cada detector de descontinuidades. 


\subsubsection{Aplicação do Método Híbrido - 1D}

A Tabela 4.1 mostra uma comparação entre o método híbrido com o detector desenvolvido e o método WENO para o cálculo da primeira derivada da função Cosseno (4.1). Com a derivada calculada numericamente e com a solução exata, que é dada por exata $=-\pi \operatorname{sen}(\pi x)$, é posível determinar o erro obtido em cada método com erro $=\mid$ exata - numérica $\mid$.

\begin{tabular}{|l|c|c|}
\cline { 2 - 3 } \multicolumn{1}{c|}{} & \multicolumn{2}{c|}{ Erro } \\
\hline$N_{x}$ & WENO & Híbrido \\
\hline 81 & $3.8000 \mathrm{E}-4$ & $3.1400 \mathrm{E}-4$ \\
\hline 101 & $1.9500 \mathrm{E}-4$ & $2.4300 \mathrm{E}-5$ \\
\hline 201 & $2.4000 \mathrm{E}-5$ & $3.7859 \mathrm{E}-6$ \\
\hline 401 & $3.0000 \mathrm{E}-5$ & $1.9885 \mathrm{E}-6$ \\
\hline
\end{tabular}

Tabela 4.1: Tabela de erros - Derivada da função Cosseno - 1D

Nota-se que, como a função e suas derivadas são contínuas, o detector considera todas as regiões como sendo suaves. Sendo assim, o método numérico híbrido aplicará apenas o método de diferenças centrais. Para os diversos números de pontos na discretização, o erro do método WENO é maior quando comparado com o híbrido, que aplica apenas o método de diferenças centrais.

\subsection{Equações de Euler - 1D}

Com o desenvolvimento do novo detector de descontinuidades e os testes realizados para diversas funções unidimensionais, é possível aplicar o método numérico híbrido para resolver as equações de Euler 1D. Através da solução dessas equações, foram construídos gráficos que permitem visualizar e comparar a solução numérica do método híbrido desenvolvido com a solução calculada apenas através do método WENO.

As equações de Euler 1D na forma vetorial são dadas por

$$
\frac{\partial \mathbf{U}}{\partial t}+\frac{\partial \mathbf{F}}{\partial x}=\mathbf{0}
$$

onde

$$
\mathbf{U}=\left[\begin{array}{c}
\rho \\
\rho u_{x} \\
\rho e_{T}
\end{array}\right], \quad \mathbf{F}=\left[\begin{array}{c}
\rho u_{x} \\
p+\rho u_{x}^{2} \\
u_{x}\left(\rho e_{T}+p\right)
\end{array}\right]
$$

Nota-se pelos vetores apresentados na equação (4.7) que há três equações e quatro incógnitas: densidade $(\rho)$, velocidade $\left(u_{x}\right)$, pressão $(p)$ e energia total específica $\left(e_{T}\right)$. Assim, é necessário 
construir uma relação entre duas incógnitas que permite resolver as equações de Euler, sendo

$$
p=(\gamma-1)\left(\rho e_{T}-\frac{1}{2} \rho u_{x}^{2}\right)
$$

Para resolver a derivada espacial são utilizados os métodos híbrido e WENO, já para resolver a derivada temporal utiliza-se do método Runge-Kutta TVD de ordem 3 presente em [13]. Além disso, com a finalidade de resolver as equações de Euler numericamente, realiza-se a separação de fluxo apresentada em [28]. As equações de Euler para o caso unidimensional são detalhadas no apêndice B.

Com as equações de Euler para o caso unidimensional, é possível resolvê-las numericamente para uma determinada condição inicial. Com os resultados obtidos, são construídos gráficos que permitem comparar a solução do método híbrido com o WENO. Isso permite avaliar o desempenho de cada método e analisar suas principais vantagens e desvantagens.

A condição inicial considerada para as equações de Euler 1D é:

- $\left(\rho, u_{x}, p\right)=\left\{\begin{array}{ll}(3.857143,2.629369,10.33333), & \text { se } x<-4 \\ (1+0.2 \operatorname{sen}(5 x), 0,1), & \text { se } x>-4\end{array} \quad x \in[-5,5]\right.$

Para realizar a implementação das equações de Euler, foi considerada a variável $N_{x}$ que representa o número de pontos utilizados na discretização, sendo que a variável $T$ representa o tempo final.

A Figura 4.11 apresenta a condição inicial utilizada para resolver numericamente as equações de Euler unidimensional.

A Figura 4.12 mostra os resultados obtidos com o método híbrido para a densidade $(\rho)$, pressão $(p)$, velocidade $\left(u_{x}\right)$ e energia total específica $\left(e_{T}\right) \operatorname{com} N_{x}=201, C F L=0.2$ e $T=1.8$. A solução de referência é calculada com o método WENO para $N_{x}=1601$.

Nota-se, de uma forma geral, que o método híbrido não obtém uma solução próxima da referência, como pode ser observada pelas Figuras 4.12(a) e 4.12(d). Isso acontece pois o número de pontos utilizados na discretização é pequeno, afetando diretamente na solução numérica calculada.

O mesmo não ocorre para $N_{x}=801$, ou seja, quando são utilizados mais pontos na discretização. A Figura 4.13 mostra os resultados para a densidade $(\rho)$, pressão $(p)$, velocidade $\left(u_{x}\right)$ e energia total específica $\left(e_{T}\right)$, permitindo a comparação com a solução de referência.

Observa-se que o resultado obtido é muito próximo da solução de referência para todas as variáveis, como pode ser visto principalmente nas Figuras 4.13(a) e 4.13(d).

A Figura 4.14 mostra os resultados quando o número de pontos na discretizção é diferente para os métodos WENO e híbrido, sendo $N_{x}=801$ e $N_{x}=601$, respectivamente. Com esses gráficos, pode-se comparar a solução numérica calculada quando o número de pontos é menor para o método híbrido. 


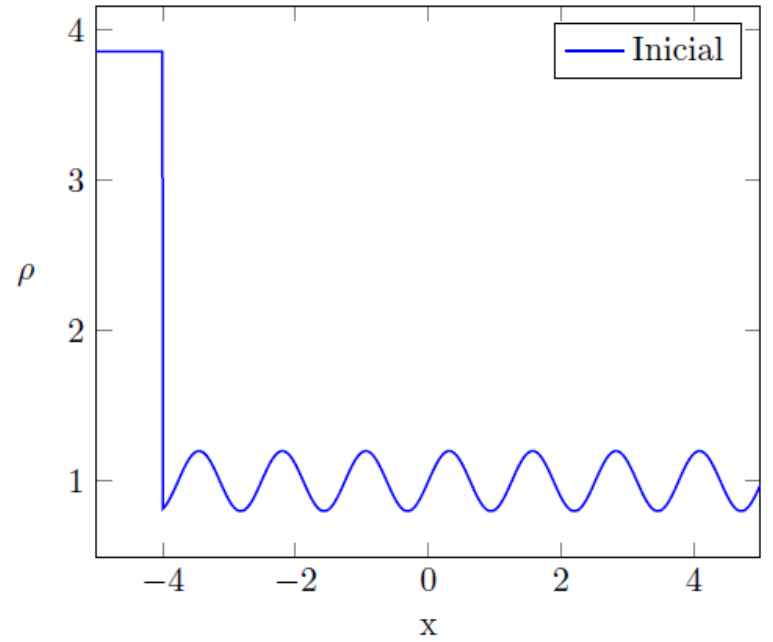

(a) Densidade

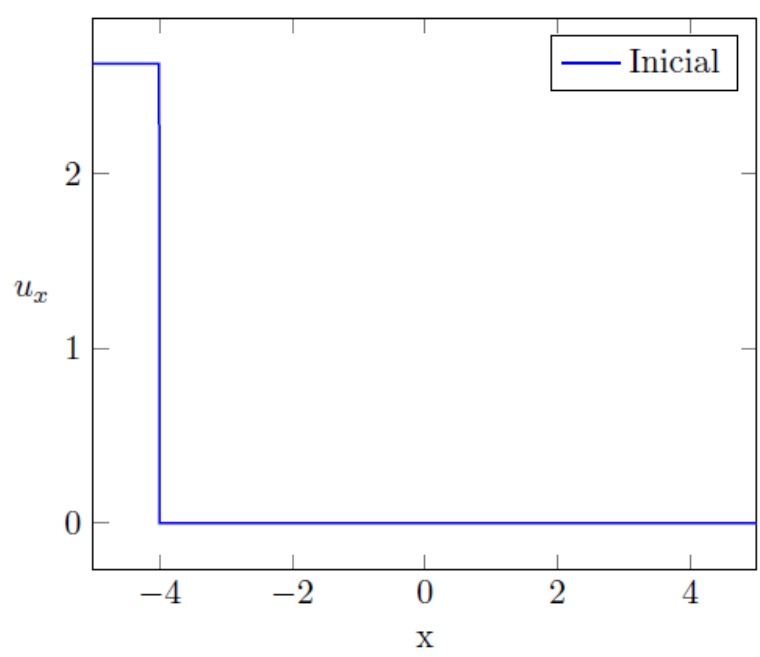

(c) Velocidade

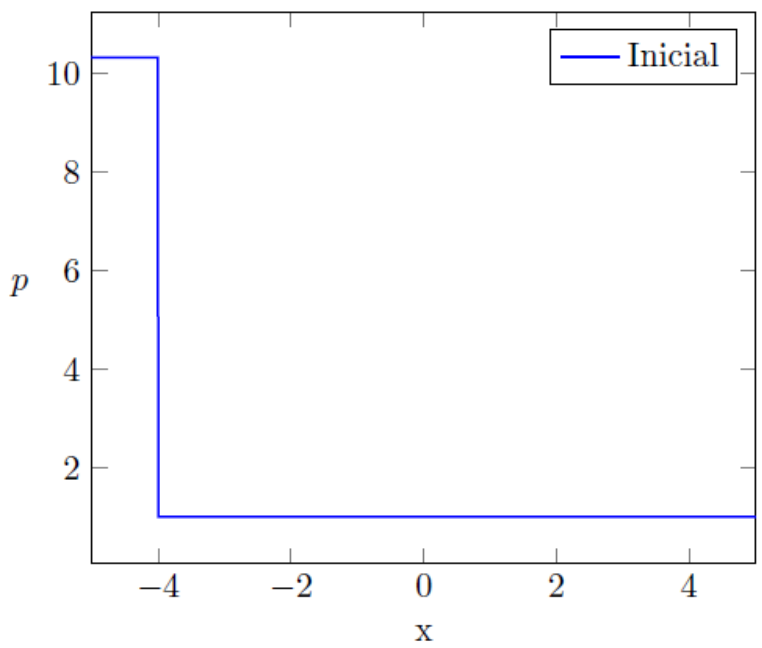

(b) Pressão

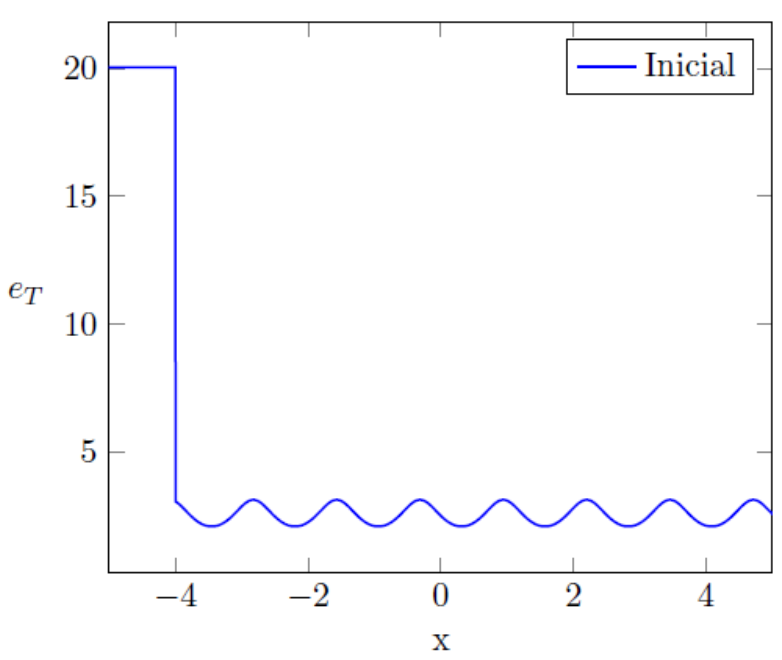

(d) Energia

Figura 4.11: Euler 1D - Condição Inicial - $T=0$

Nota-se que os resultados para todas as variáveis são muito próximos, tendo uma pequena diferença no cálculo da densidade $(\rho)$, como pode ser visto pela Figura 4.14(a) entre $x \in[0,2]$. Esses gráficos mostram que e o método numérico híbrido com o detector desenvolvido obtém uma solução muito próxima do método WENO utilizando menos pontos na discretização.

Apesar de apenas 201 pontos na discretização não ser uma quantidade suficiente para que as soluções obtidas pelos métodos híbrido e WENO contenham os detalhes necessários, a Figura 4.15 mostra o resultado para ambos os métodos com $N_{x}=201$. Esses gráficos permitem comparar as soluções numéricas calculadas.

Observa-se pelas Figuras 4.15(a) e 4.15(b) que o método híbrido consegue obter uma solução com mais detalhes e mais próxima da solução de referência, já que as ondas presentes nessa região para a variável densidade são melhores detalhadas. Já para a pressão, velocidade e energia, o resultado obtido é bem próximo quando se compara o método WENO com o método híbrido. 


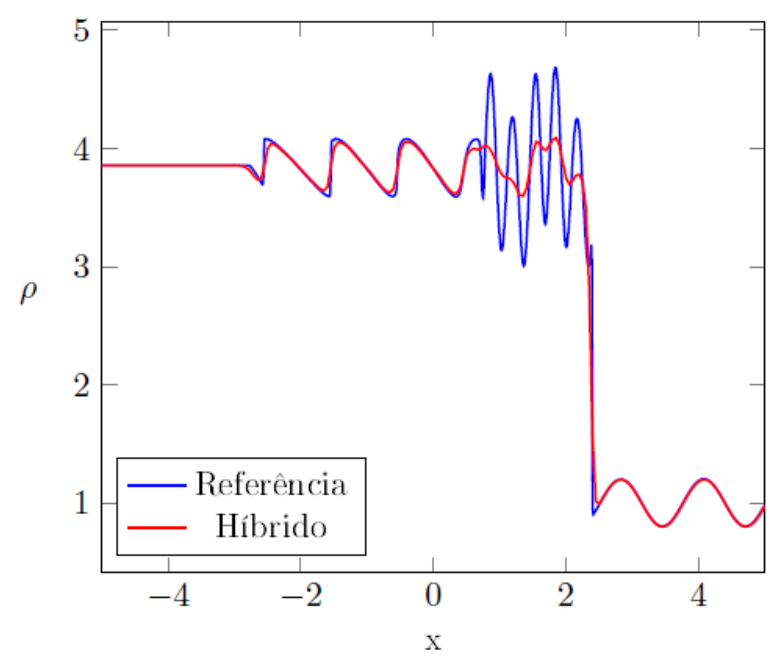

(a) Densidade

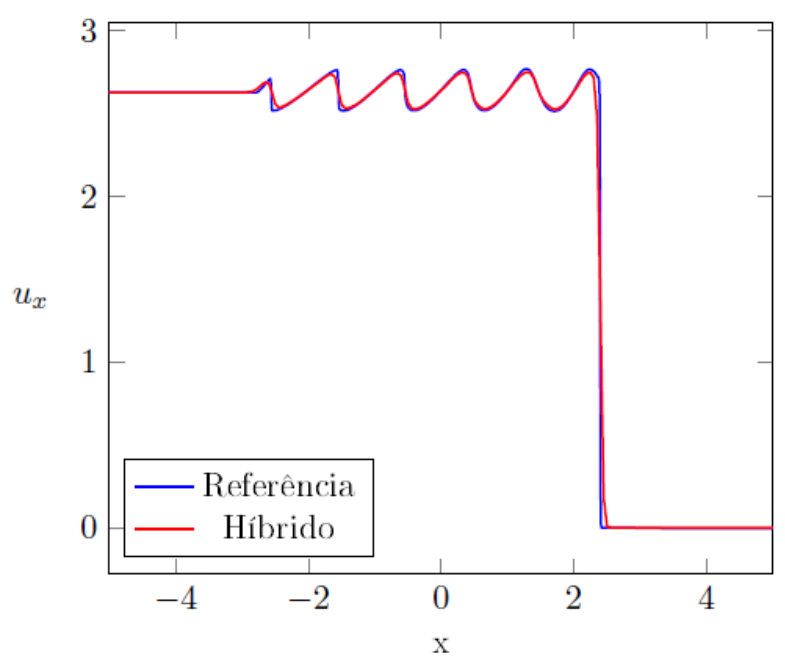

(c) Velocidade

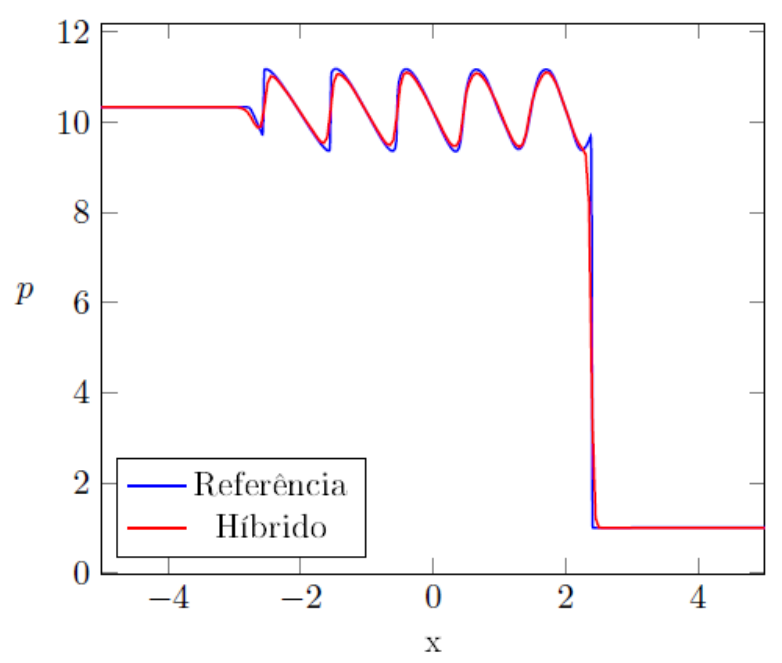

(b) Pressão

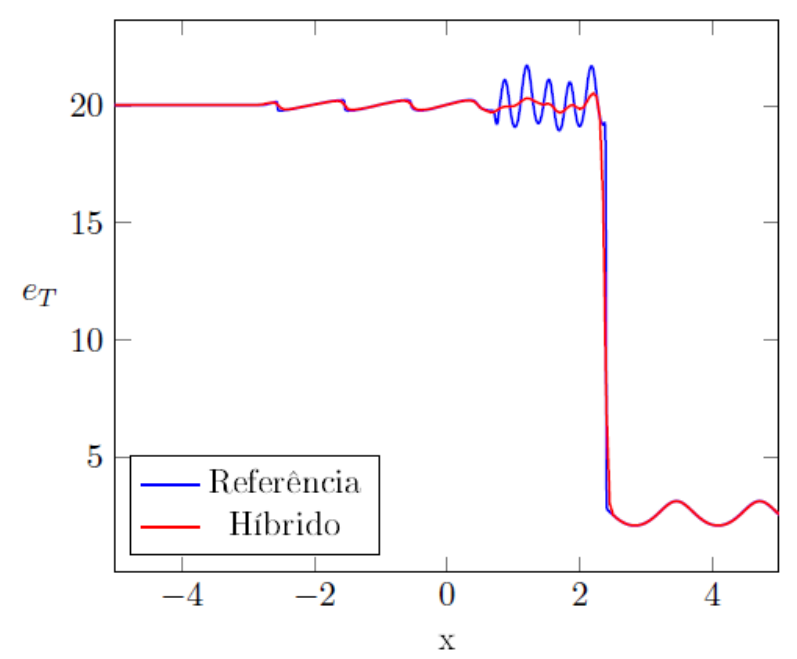

(d) Energia

Figura 4.12: Euler 1D - Híbrido - $N_{x}=201, C F L=0.2$ e $T=1.8$

A Figura 4.16 mostra uma comparação do método WENO com o método híbrido quando ambos utilizam o mesmo número de pontos na discretização, sendo nesse caso $N_{x}=801$.

Nota-se que para um mesmo número de pontos, o resultado obtido para a densidade, pressão, velocidade e energia são muito próximos, ocorrendo uma pequena diferença em $\rho$ como pode ser visto pelas Figuras 4.16(a) e 4.16(b). A principal vantagem do método híbrido consiste em aplicar o método WENO apenas nas regiões que são consideradas como sendo descontínuas.

Através dos resultados calculados com os métodos WENO e híbrido para as equações de Euler, a Tabela 4.2 mostra o tempo computacional consumido em cada método para quantidades diferentes de pontos na discretização, com tempo final $T=1.8$ e $C F L=0.2$. 


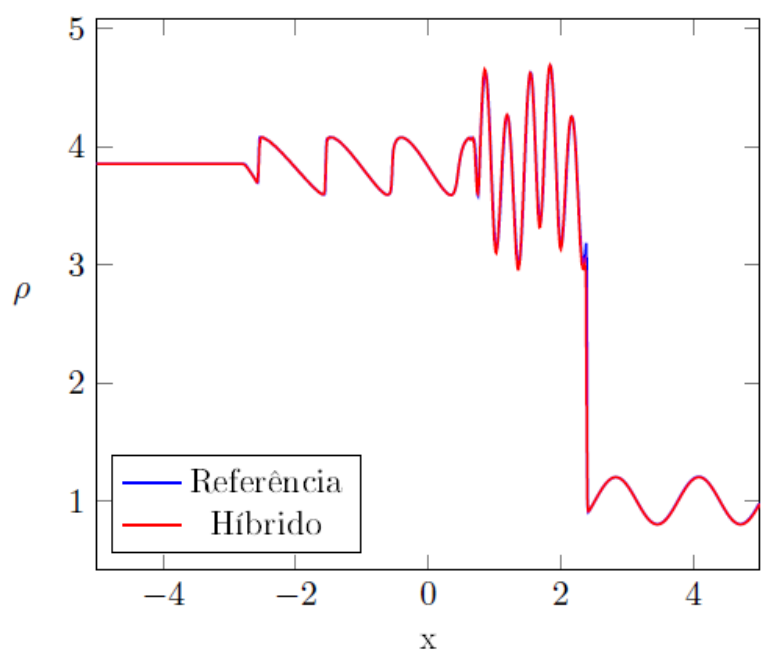

(a) Densidade

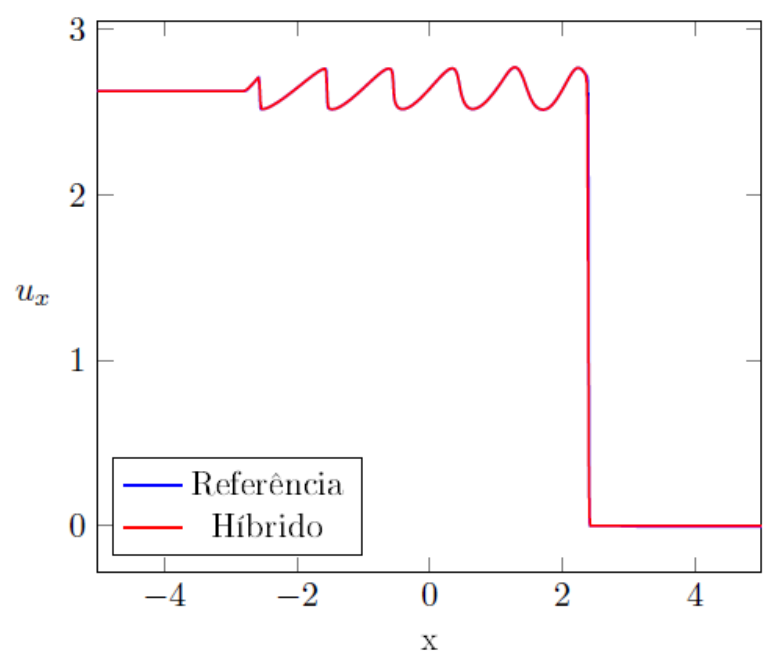

(c) Velocidade

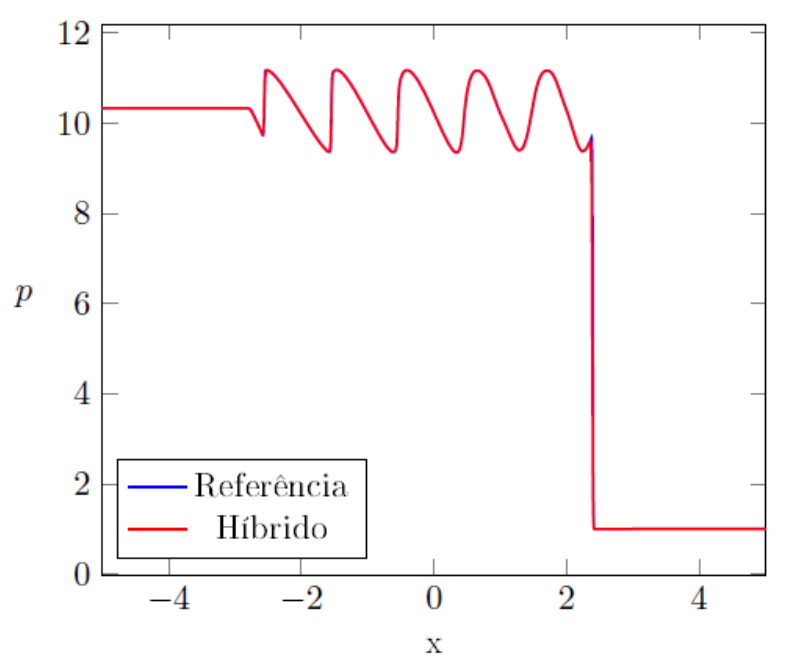

(b) Pressão

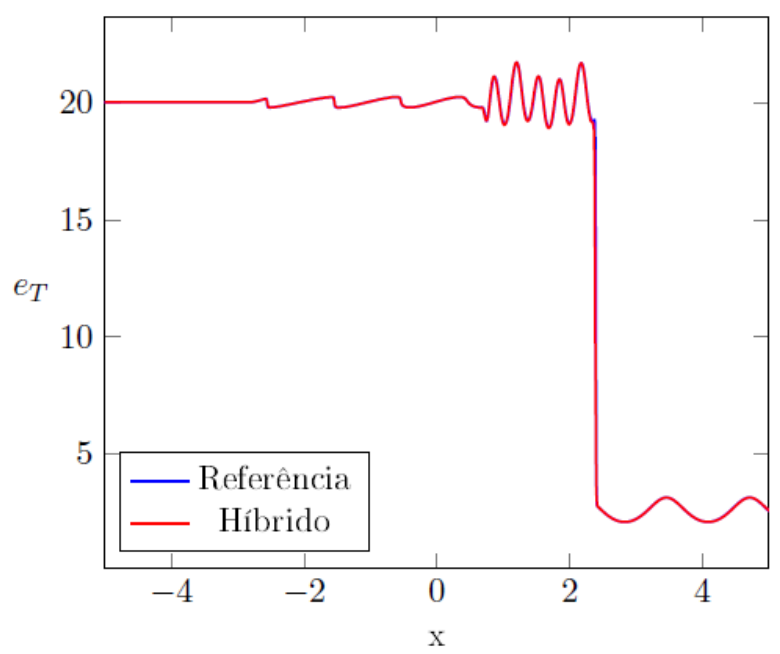

(d) Energia

Figura 4.13: Euler 1D - Híbrido - $N_{x}=801, C F L=0.2$ e $T=1.8$

\begin{tabular}{|l|l|l|}
\cline { 2 - 3 } \multicolumn{1}{c|}{} & \multicolumn{2}{c|}{ Tempo } \\
\hline$N_{x}$ & WENO & Híbrido \\
\hline 201 & $0.754 s$ & $0.766 s$ \\
\hline 401 & $1.849 s$ & $1.889 s$ \\
\hline 601 & $3.669 s$ & $3.703 s$ \\
\hline 801 & $6.171 s$ & $6.335 s$ \\
\hline 1601 & $22.275 s$ & $23.235 s$ \\
\hline
\end{tabular}

Tabela 4.2: Tabela de tempos de execução - Euler 1D

Observa-se que os tempos computacionais são muito próximos, porém o método híbrido com o detector desenvolvido leva mais tempo para o cálculo da solução das equações de Euler quando comparado com o método WENO. Apesar de levar mais tempo com o mesmo número de pontos na discretização, o método híbrido necessita de menos pontos para obter a mesma qualidade do 


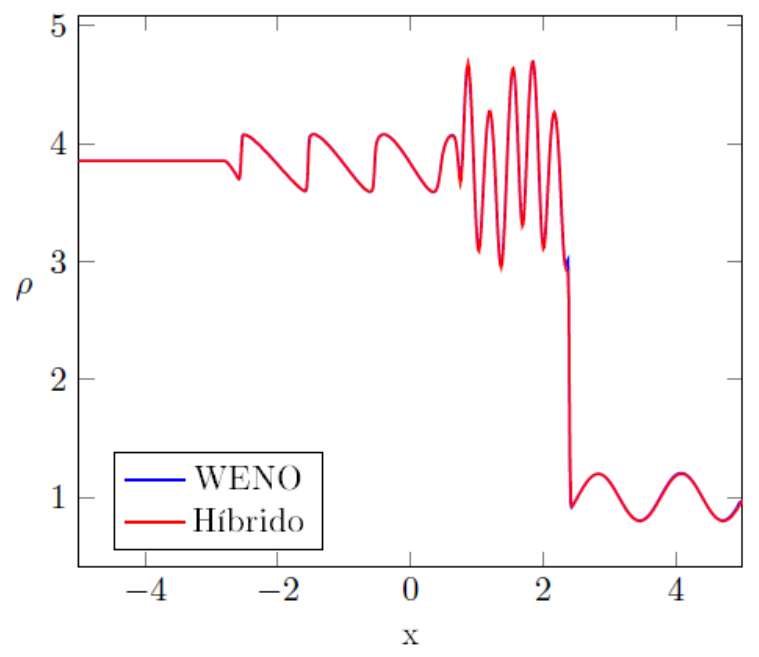

(a) Densidade

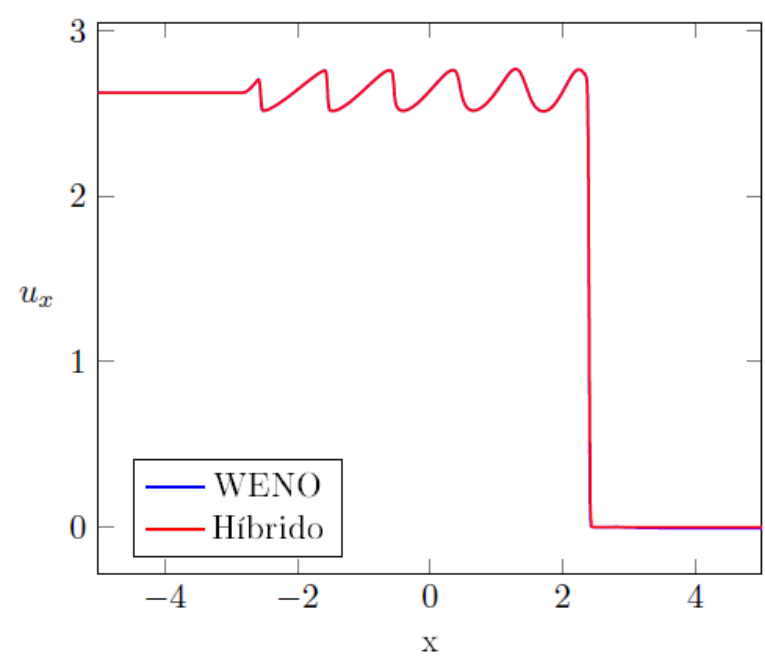

(c) Velocidade

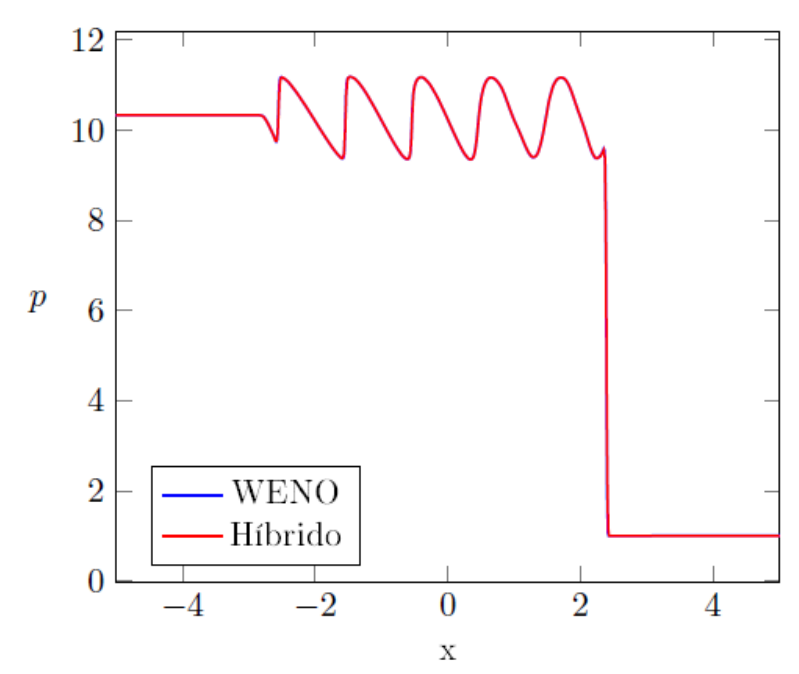

(b) Pressão

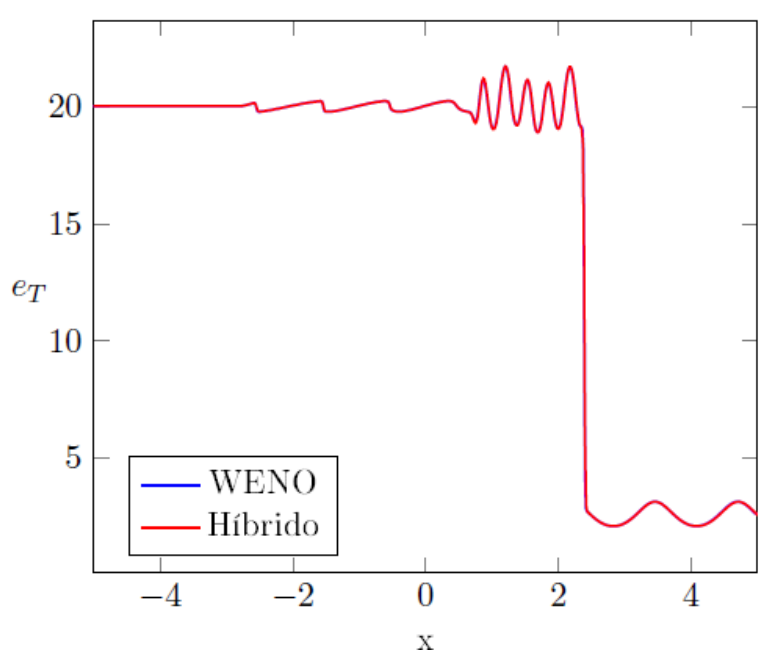

(d) Energia

Figura 4.14: Euler 1D - WENO e Híbrido - $C F L=0.2$ e $T=1.8$

método WENO, como pode ser visto na Figura 4.14, uma vez que o tempo computacional para o método híbrido com $N_{x}=601$ é $3.703 s$, e para o método WENO com $N_{x}=801$ o tempo gasto é de $6.171 s$.

\subsection{Detector Desenvolvido - 2D}

Com a intenção de mostrar o funcionamento do detector desenvolvido para funções bidimensionais, foram consideradas diversas situações que permitem avaliar se a nova função detectora determina corretamente as regiões que apresentam função suave ou descontínua. Sendo assim, foram testadas uma função contínua com derivadas contínuas e dois casos que apresentam descontinuidades na própria função. 


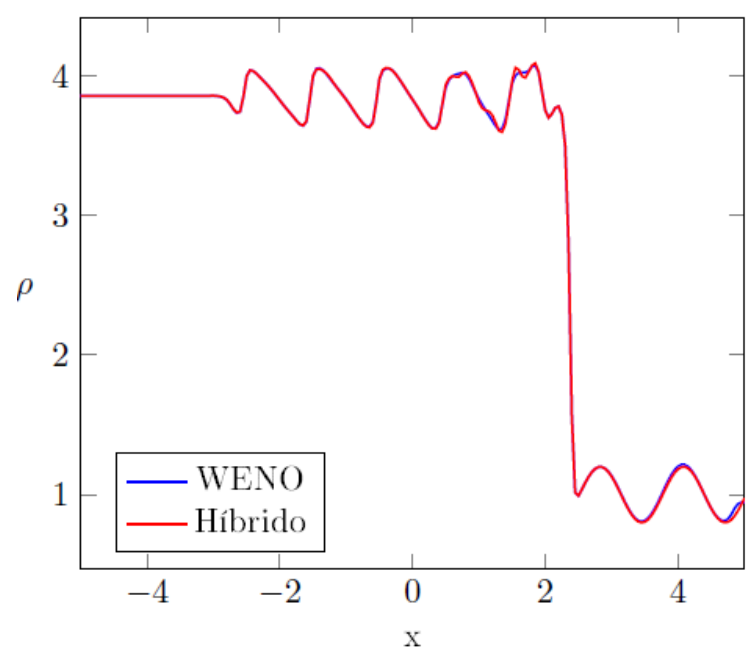

(a) Densidade

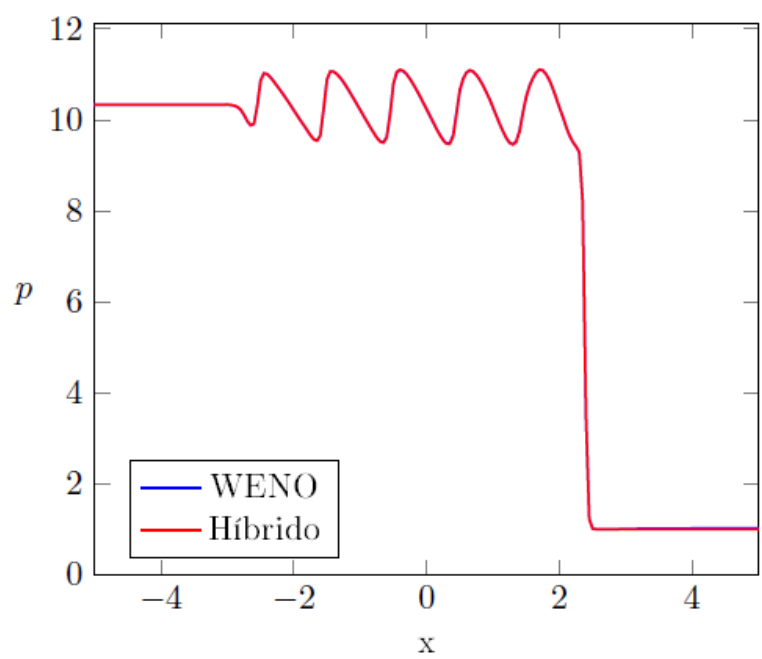

(c) Pressão

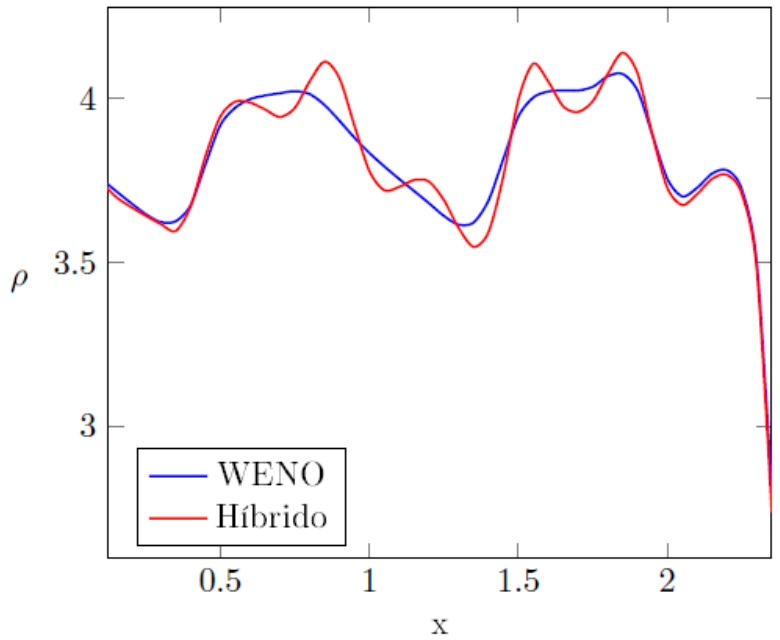

(b) Densidade - Ampliação

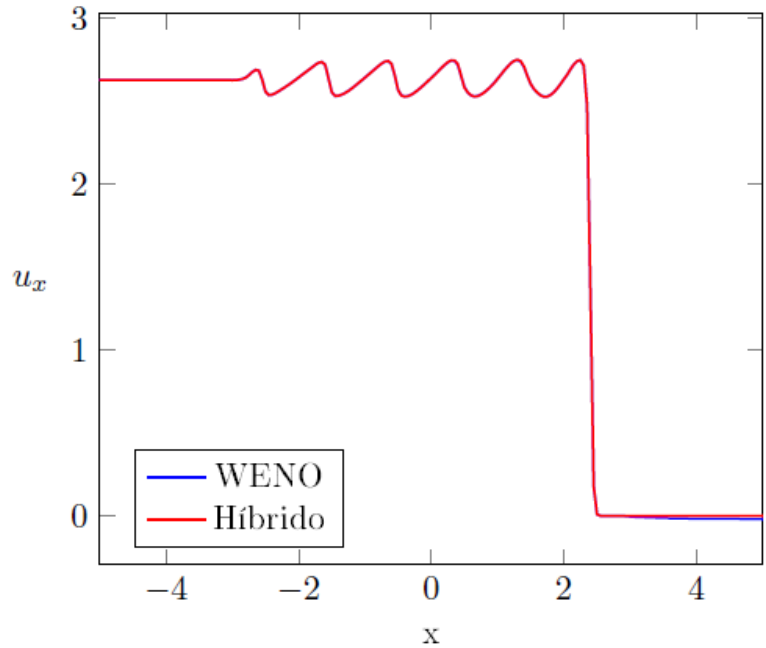

(d) Velocidade

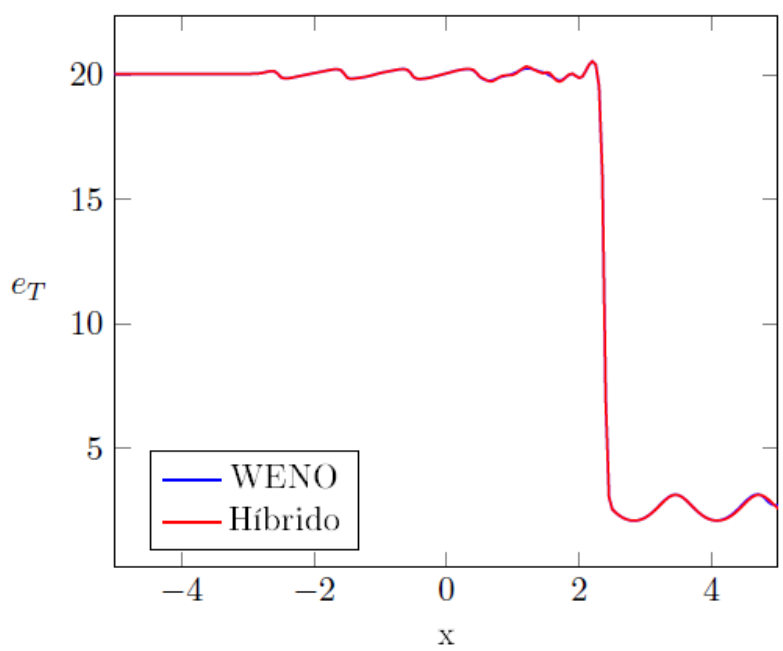

(e) Energia

Figura 4.15: Comparação 1D - WENO e Híbrido - $N_{x}=201, C F L=0.2$ e $T=1.8$ 


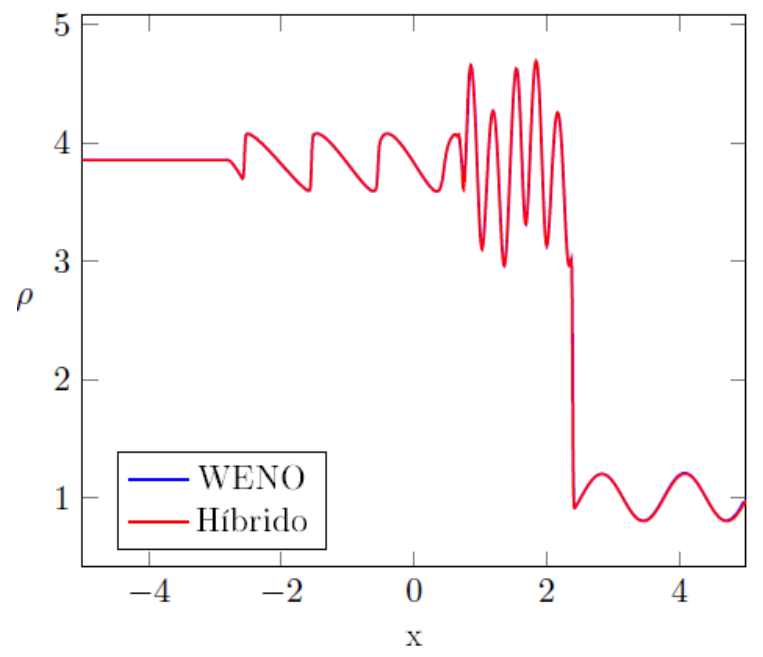

(a) Densidade

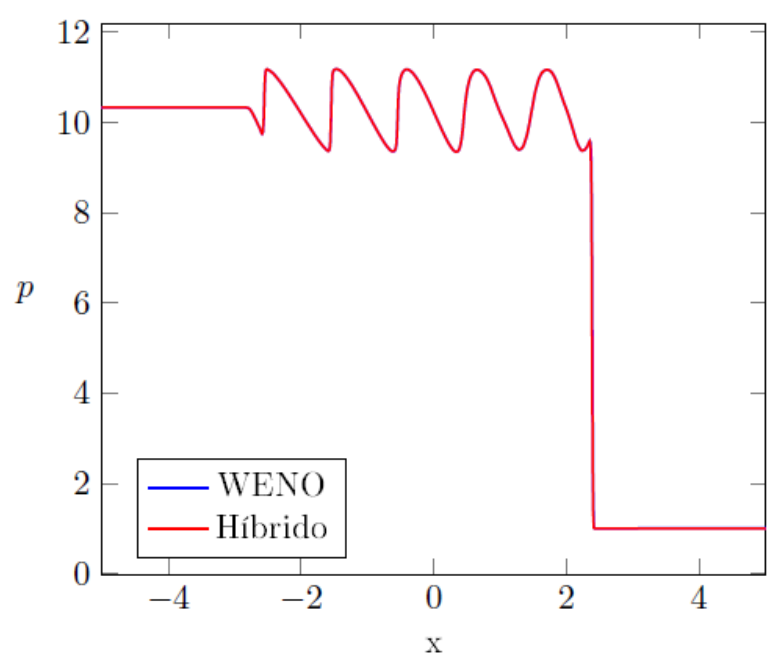

(c) Pressão

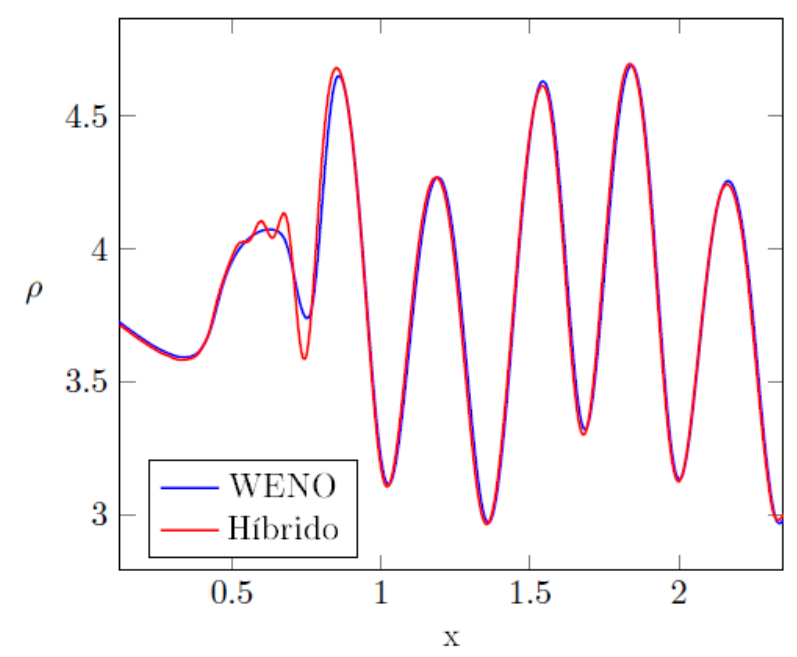

(b) Densidade - Ampliação

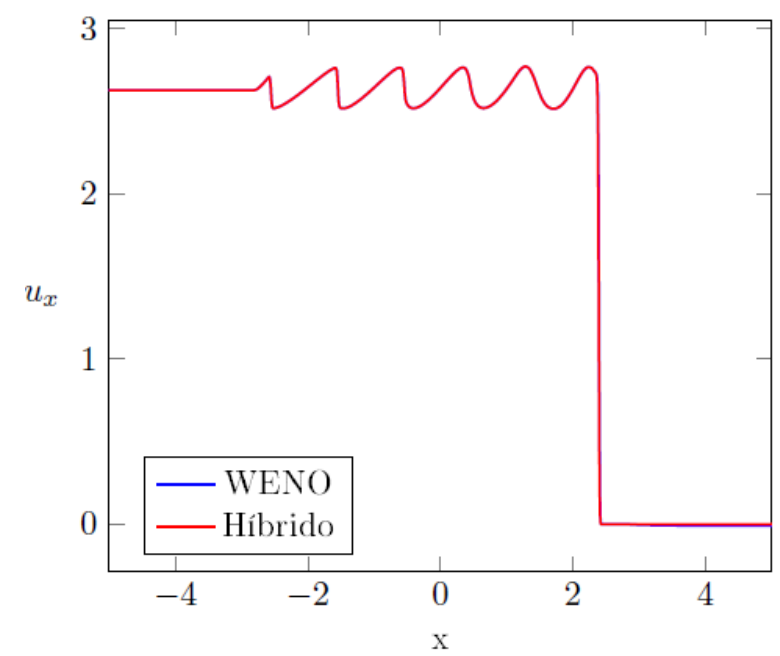

(d) Velocidade

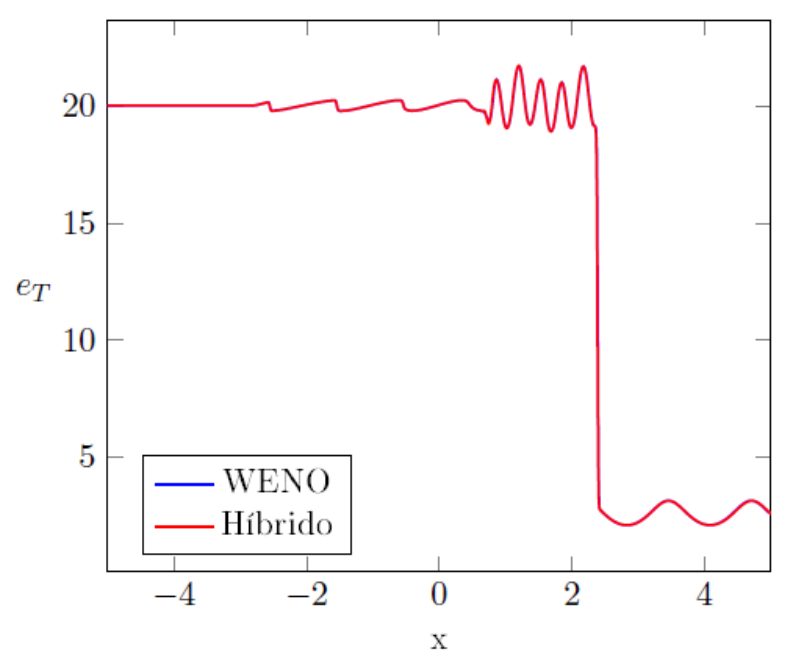

(e) Energia

Figura 4.16: Comparação 1D - WENO e Híbrido - $N_{x}=801, C F L=0.2$ e $T=1.8$ 
Para determinar os pesos $\sigma$, o detector é aplicado em cada direção ( $x$ e $y$ ) de forma a obter duas matrizes $\left(\sigma_{x}\right.$ e $\sigma_{y}$ ) que representam se uma determinada região pode ser considerada com função suave ou descontínua.

As funções bidimensionais consideradas foram:

- Função Suave:

$$
f(x, y)=x^{2}+y^{3}-1, \quad x \in[-2,2]
$$

- Função Descontínua - Circunferência:

$$
f(x, y)=\left\{\begin{array}{ll}
1, & \text { se } x^{2}+y^{2} \leq 1 \\
0, & \text { caso contrário }
\end{array}, \quad x \in[-2,2]\right.
$$

- Função Descontínua - Triângulo:

$$
f(x, y)=\left\{\begin{array}{ll}
0, & \text { se } y<2 x-2 \\
1, & \text { se } y<-2 x-2 \\
2, & \text { se } y \geq 2 x-2 \text { e } y \geq-2 x-2
\end{array}, \quad x \in[-2,2]\right.
$$

\subsubsection{Suave}

A Figura 4.17 mostra os resultados do novo detector desenvolvido para uma função contínua (4.9) que apresenta derivadas contínuas. O número de pontos utilizados na discretização são $N_{x}=$ 101 pontos na direção $x$ e $N_{y}=101$ pontos na direção $y$.

Os resultados para os pesos $\sigma_{x}$ e $\sigma_{y}$ podem ser vistos nas Figuras 4.17(b) e 4.17(c), respectivamente. Observa-se que, como a função e suas derivadas são contínuas, é esperado que os pesos calculados, tanto na direção $x$ como na direção $y$, possuam valores 1 , indicando assim que a função é suave em qualquer região.

\subsubsection{Descontínua - Circunferência}

A Figura 4.18 mostra os resultados obtidos para a função representada na equação (4.10), sendo que esta apresenta uma descontinuidade na própria função, como pode ser observado na Figura 4.18(a).

Como existe uma descontinuidade nessa função, é esperado que os pesos $\sigma_{x}$ e $\sigma_{y}$ indiquem essa região como sendo descontínua e consequentemente com os valores $\sigma=0$ na matriz. Isso de fato acontece para $N_{x}=101$ e $N_{y}=101$, como pode ser obervado nas Figuras 4.18(b) 4.18(c), 4.18(d) e 4.18(e).

O mesmo ocorre com $N_{x}=401$ e $N_{y}=401$, ou seja, utilizando mais pontos na discretização para as direções $x$ e $y$. A Figura 4.19 apresenta os resultados para $\sigma_{x}, \sigma_{y}$ e os perfis em $y=0$ e $x=0$. 


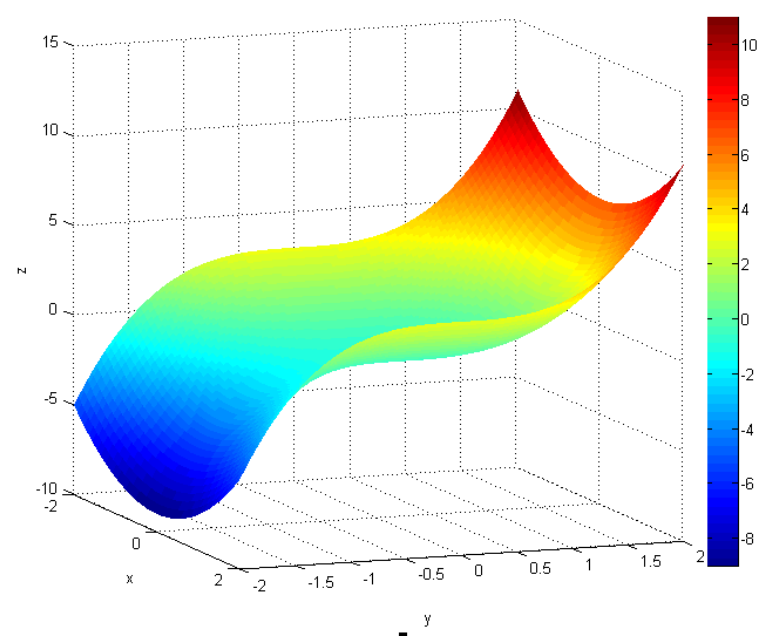

(a) Função

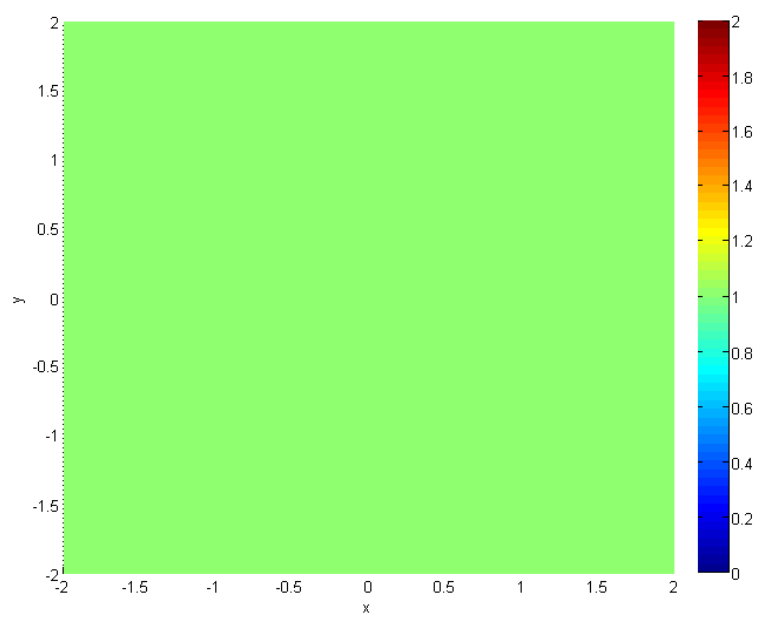

(b) Detector $-\sigma_{x}$

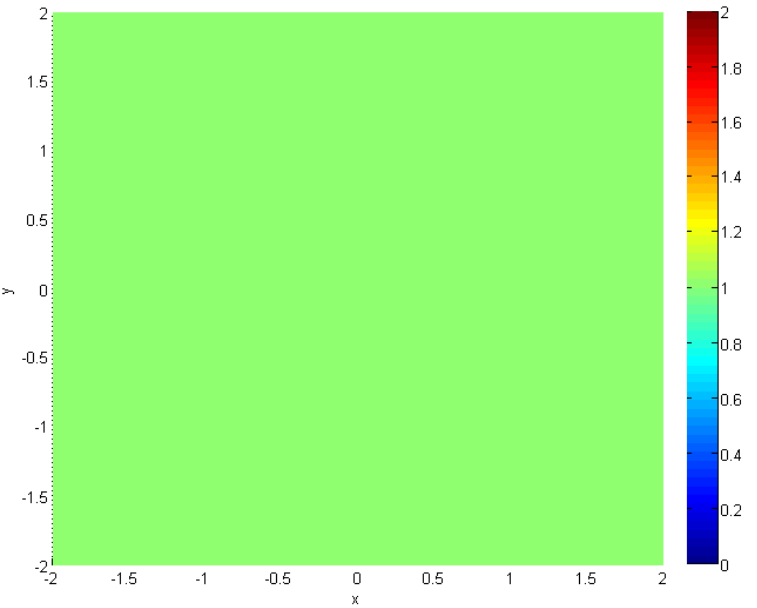

(c) Detector $-\sigma_{y}$

Figura 4.17: Novo detector 2D - Suave - $N_{x}=101$ e $N_{y}=101$ 


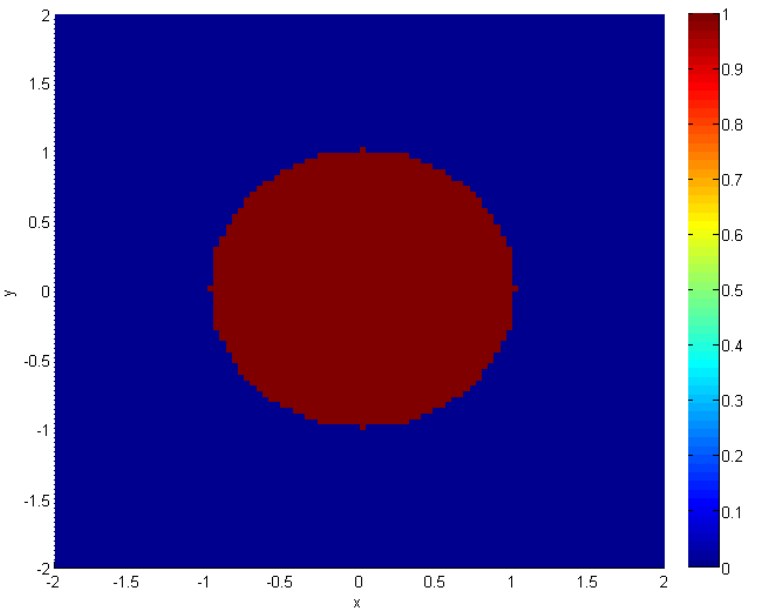

(a) Função

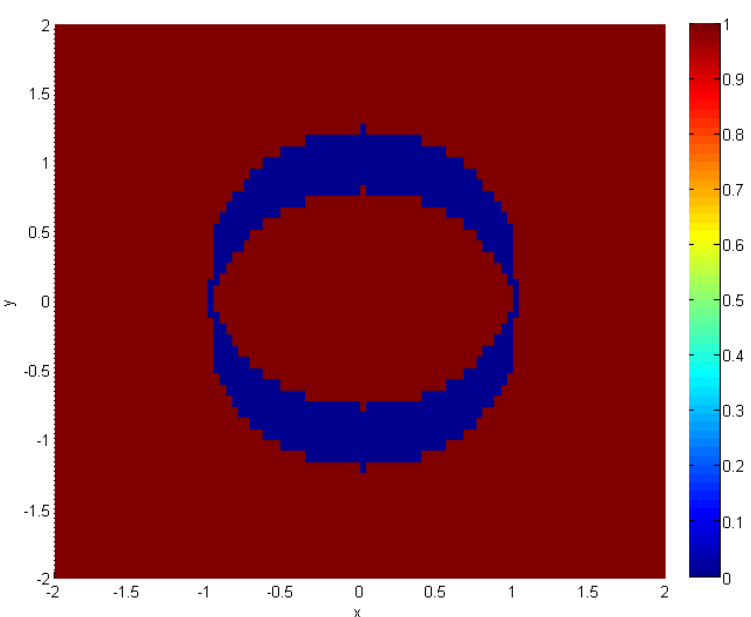

(c) Detector $-\sigma_{y}$

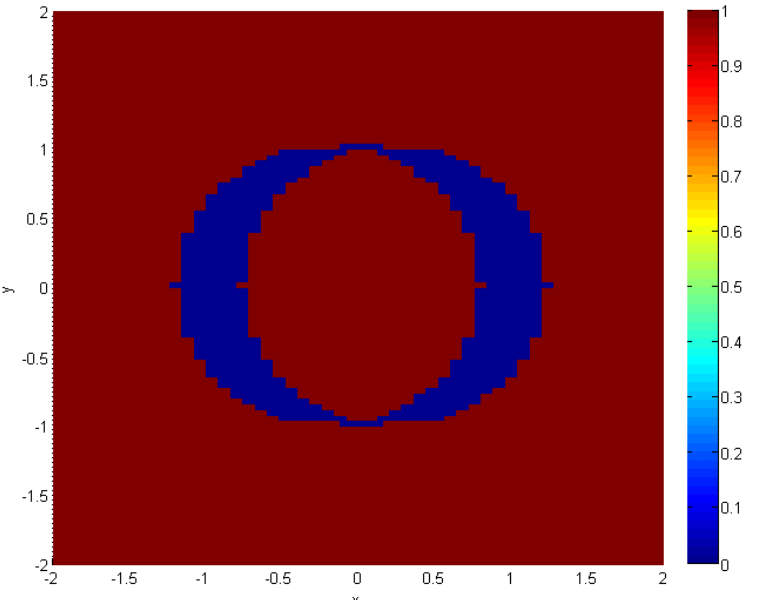

(b) Detector $-\sigma_{x}$

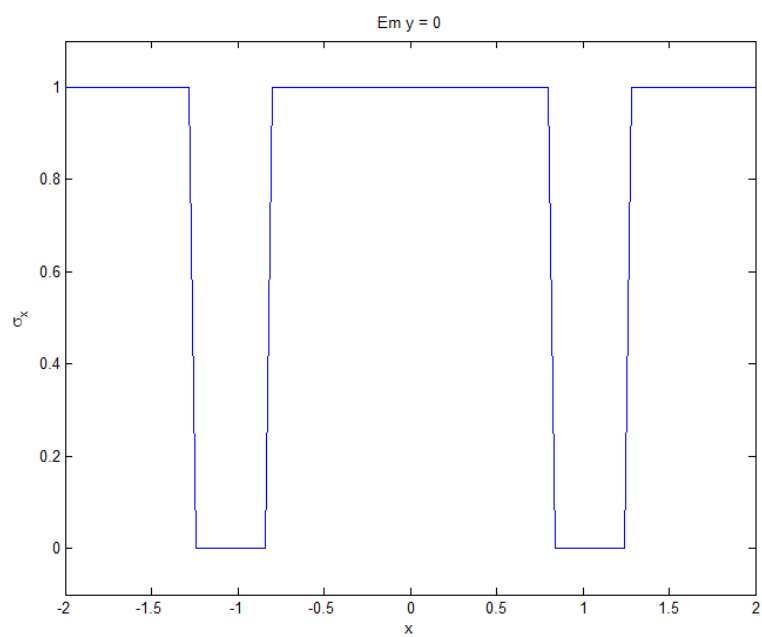

(d) Detector $-\sigma_{x}$ - Perfil em $y=0$

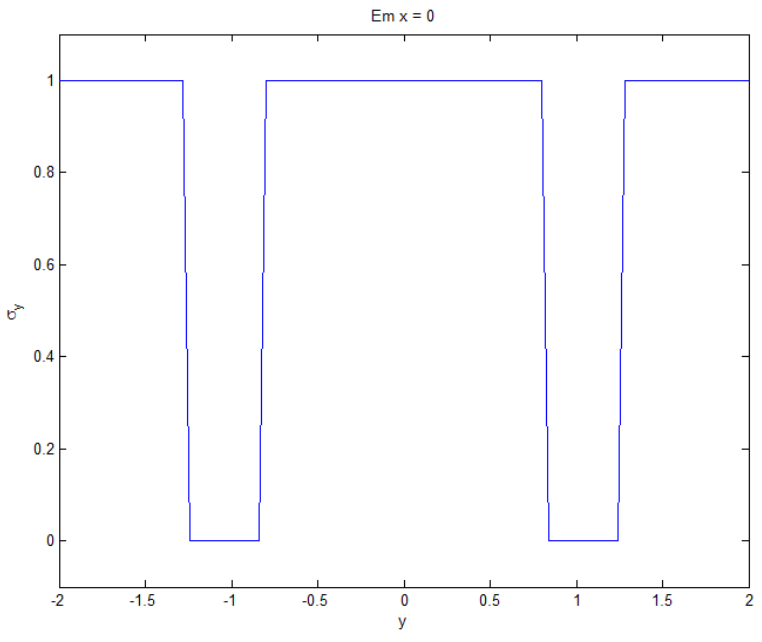

(e) Detector - $\sigma_{y}$ - Perfil em $x=0$

Figura 4.18: Novo detector 2D - Circunferência - $N_{x}=101$ e $N_{y}=101$ 


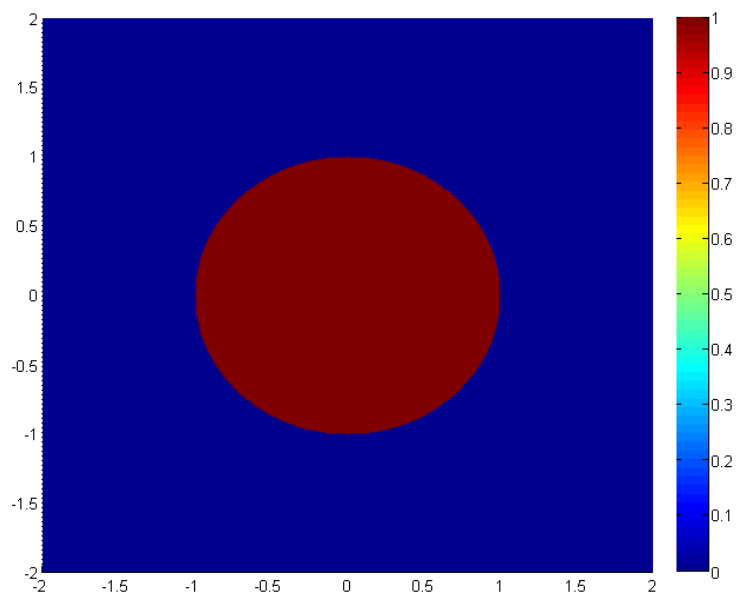

(a) Função

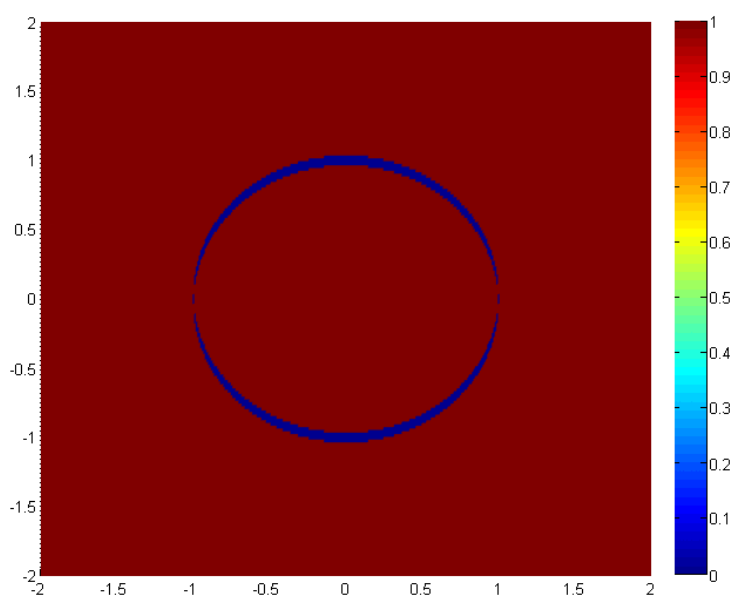

(c) Detector $-\sigma_{y}$

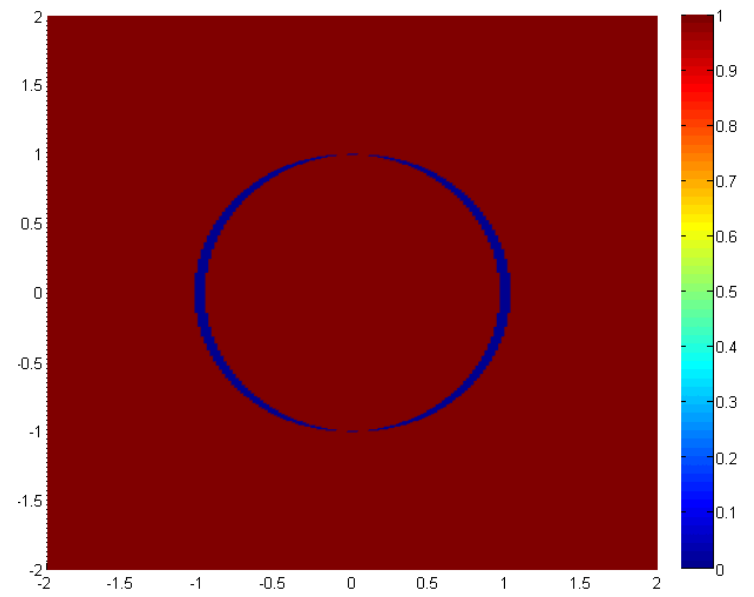

(b) Detector $-\sigma_{x}$

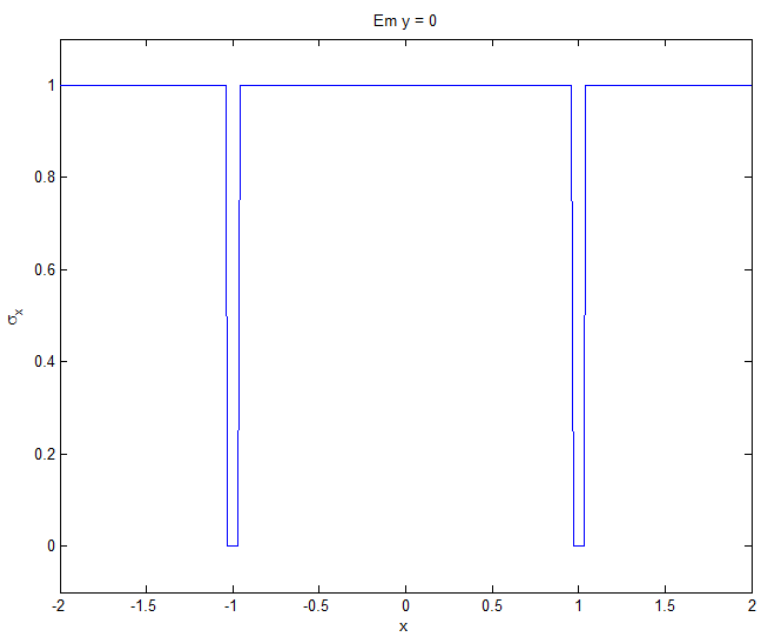

(d) Detector $-\sigma_{x}$ - Perfil em $y=0$

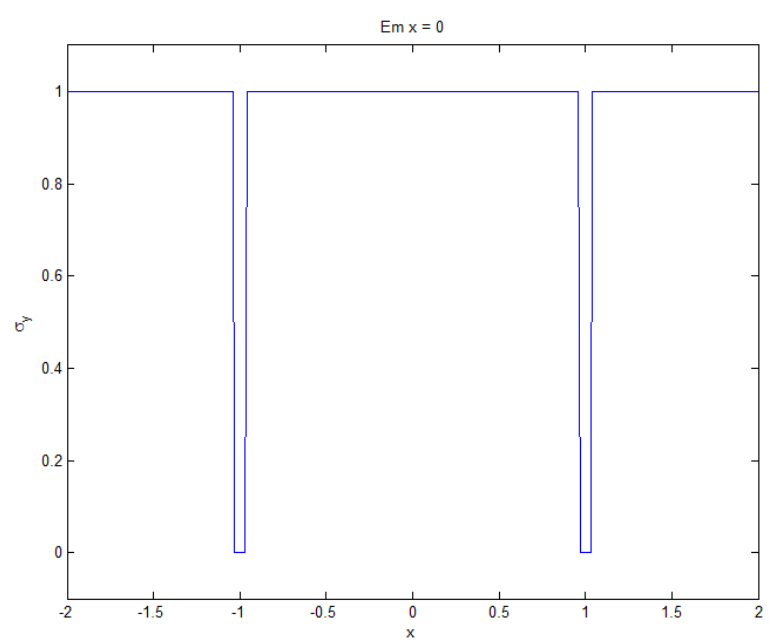

(e) Detector - $\sigma_{y}$ - Perfil em $x=0$

Figura 4.19: Novo detector 2D - Circunferência - $N_{x}=401$ e $N_{y}=401$ 


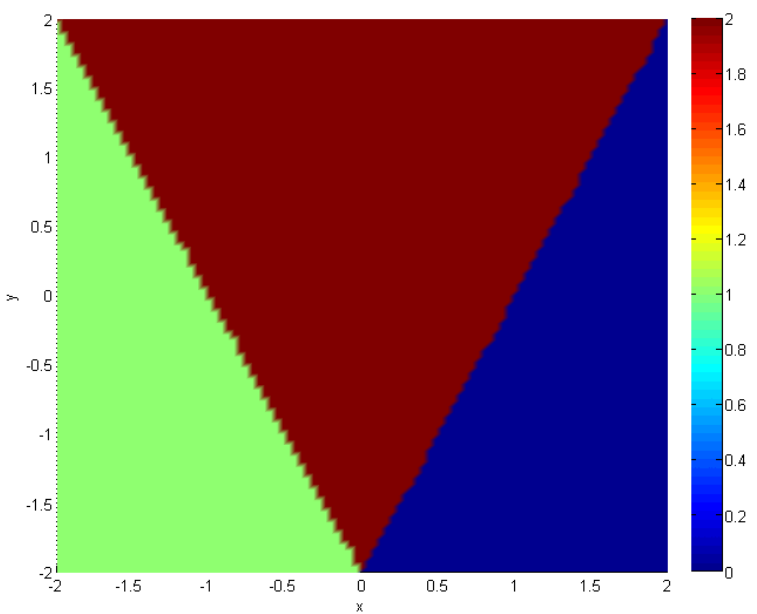

(a) Função

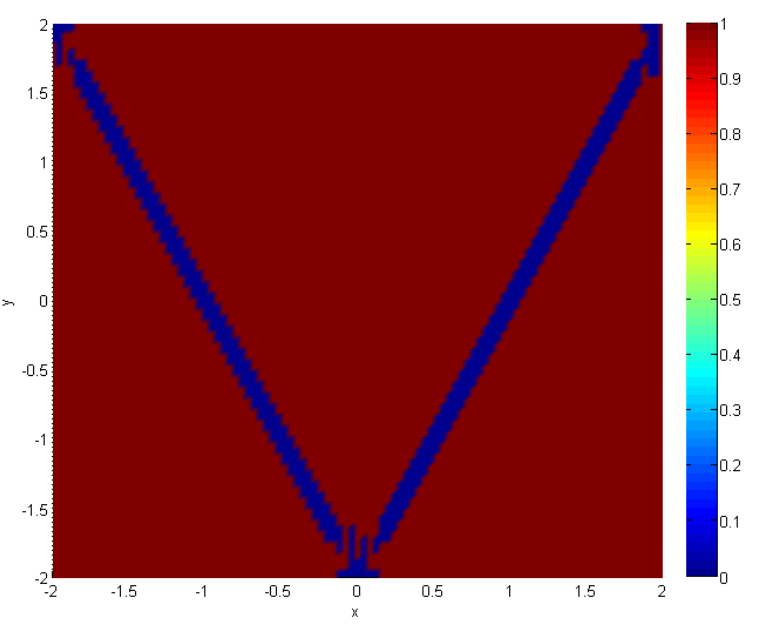

(c) Detector $-\sigma_{y}$

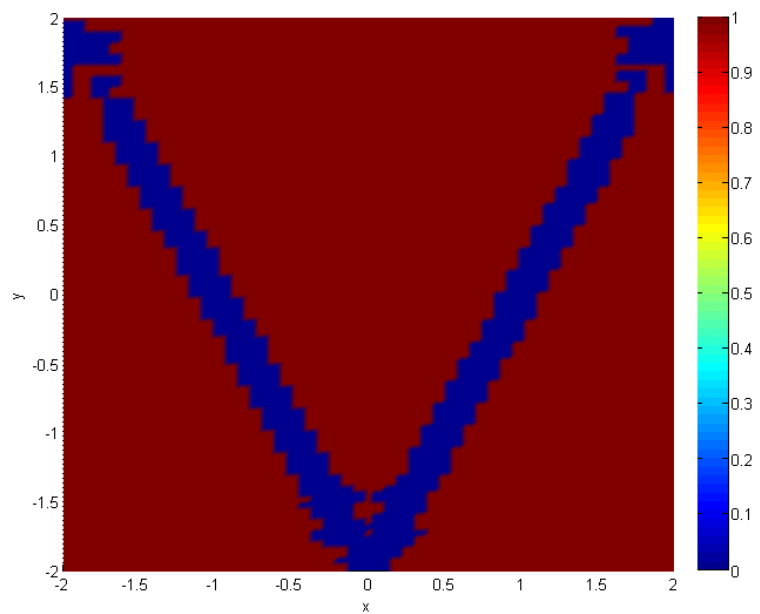

(b) Detector $-\sigma_{x}$

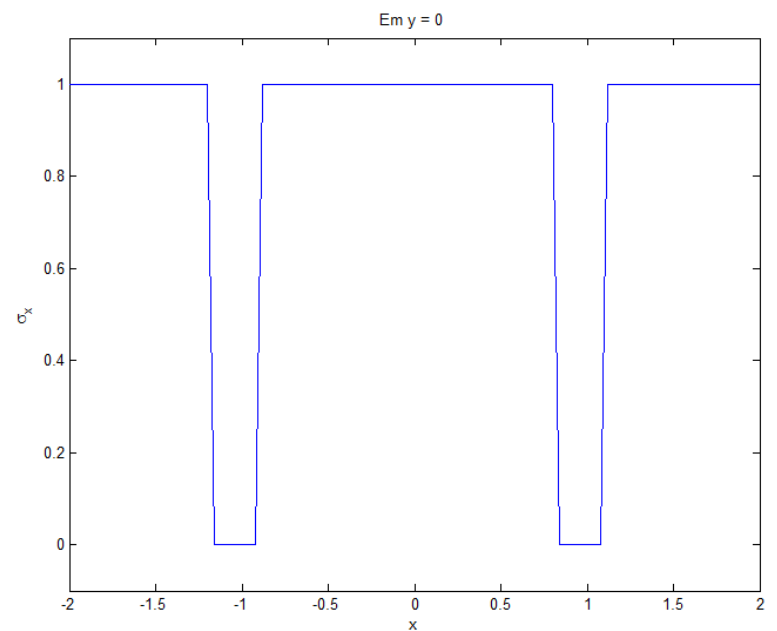

(d) Detector $-\sigma_{x}$ - Perfil em $y=0$

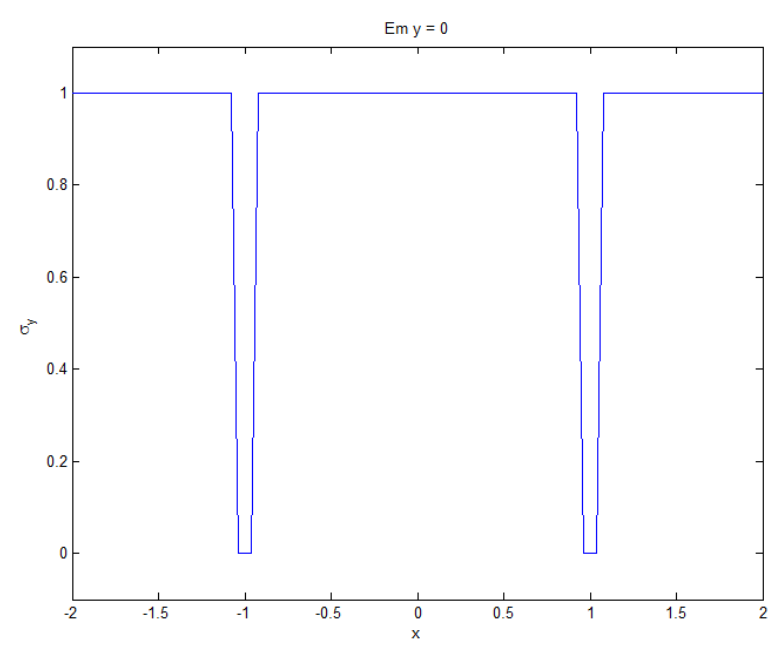

(e) Detector $-\sigma_{y}$ - Perfil em $y=0$

Figura 4.20: Novo detector 2D - Triângulo - $N_{x}=101$ e $N_{y}=101$ 


\subsubsection{Descontínua - Triângulo}

Para testar o novo detector desenvolvido, mais uma função que apresenta descontinuidades foi considerada, sendo esta representada pela equação (4.11). Dessa forma, a Figura 4.20 mostra os resultados obtidos para os pesos $\sigma_{x}$ e $\sigma_{y}$ para as direções $x$ e $y$, respectivamente.

Para $N_{x}=101$ e $N_{y}=101$, pelas Figuras 4.20(b) e 4.20(c) nota-se que os pesos $\sigma_{x}$ e $\sigma_{y}$ do novo detector desenvolvido destacam as regiões que apresentam descontinuidades. Pelos perfis representados nas Figuras 4.20(d) e 4.20(e) é possível observar a presença dessas descontinuidades e os valores obtidos com a aplicação da nova função detectora.

O mesmo exemplo é considerado para $N_{x}=401$ e $N_{y}=401$, como pode ser visto na Figura 4.21. Observa-se que para uma quantidade maior de pontos na discretização, tanto na direção $x$ como na direção $y$, os pesos $\sigma_{x}$ e $\sigma_{y}$ calculados indicam corretamente as regiões que apresentam função descontínua. Para notar de fato a descontinuidade, as Figuras 4.21(d) e 4.21(e) mostram os perfis em $y=0$.

\subsubsection{Aplicação do Método Híbrido - 2D}

As Tabelas 4.3 e 4.4 mostram os erros para o cálculo da primeira derivada da função Suave (4.9) obtidos com o método WENO e com o método híbrido. Como os métodos são aplicados em cada direção, os erros também são calculados para a direção $x$ e para a direção $y$.

\begin{tabular}{|l|c|c|}
\cline { 2 - 3 } \multicolumn{1}{c|}{} & \multicolumn{2}{c|}{ Erro } \\
\hline$N_{x} \times N_{y}$ & WENO & Híbrido \\
\hline $81 \times 81$ & $3.7900 \mathrm{E}-4$ & $2.6510 \mathrm{E}-4$ \\
\hline $101 \times 101$ & $1.1463 \mathrm{E}-4$ & $2.4797 \mathrm{E}-5$ \\
\hline $201 \times 201$ & $1.7131 \mathrm{E}-5$ & $2.3279 \mathrm{E}-6$ \\
\hline $401 \times 401$ & $1.9250 \mathrm{E}-5$ & $2.1733 \mathrm{E}-6$ \\
\hline
\end{tabular}

Tabela 4.3: Tabela de erros - Derivada da função Suave - Direção $x$ - 2D

\begin{tabular}{|l|c|c|}
\cline { 2 - 3 } \multicolumn{1}{c|}{} & \multicolumn{2}{c|}{ Erro } \\
\hline$N_{x} \times N_{y}$ & WENO & Híbrido \\
\hline $81 \times 81$ & $3.6310 \mathrm{E}-4$ & $2.8720 \mathrm{E}-4$ \\
\hline $101 \times 101$ & $1.2763 \mathrm{E}-4$ & $2.8722 \mathrm{E}-5$ \\
\hline $201 \times 201$ & $1.8745 \mathrm{E}-5$ & $2.3465 \mathrm{E}-6$ \\
\hline $401 \times 401$ & $1.5743 \mathrm{E}-5$ & $2.3467 \mathrm{E}-6$ \\
\hline
\end{tabular}

Tabela 4.4: Tabela de erros - Derivada da função Suave - Direção $y$ - 2D

Assim como para o caso unidimensional, os erros em cada direção são menores quando a derivada é calculada através do método híbrido. Isso acontece pois a função considerada é contínua com derivadas contínuas, sendo aplicado então apenas o método de diferenças centrais. 


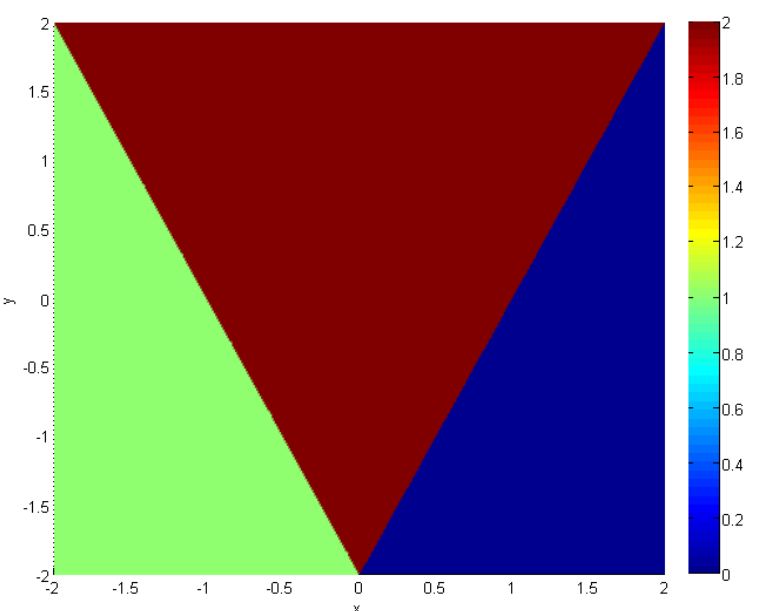

(a) Função

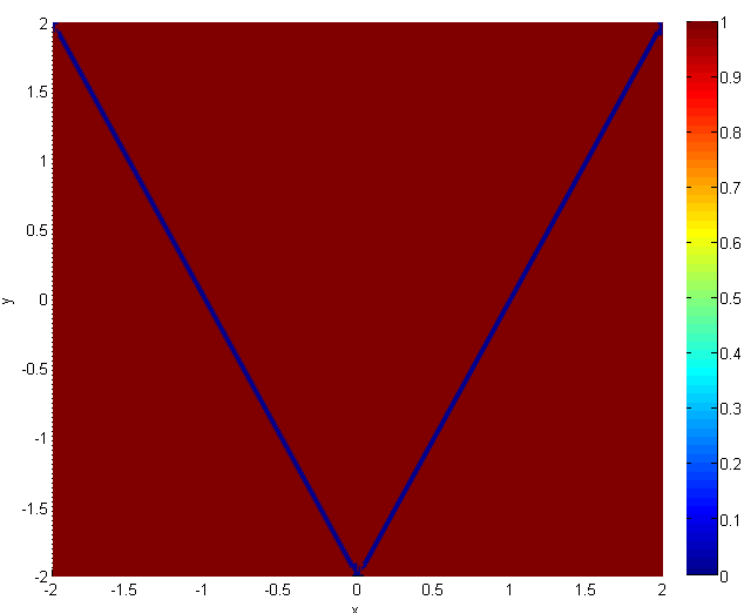

(c) Detector $-\sigma_{y}$

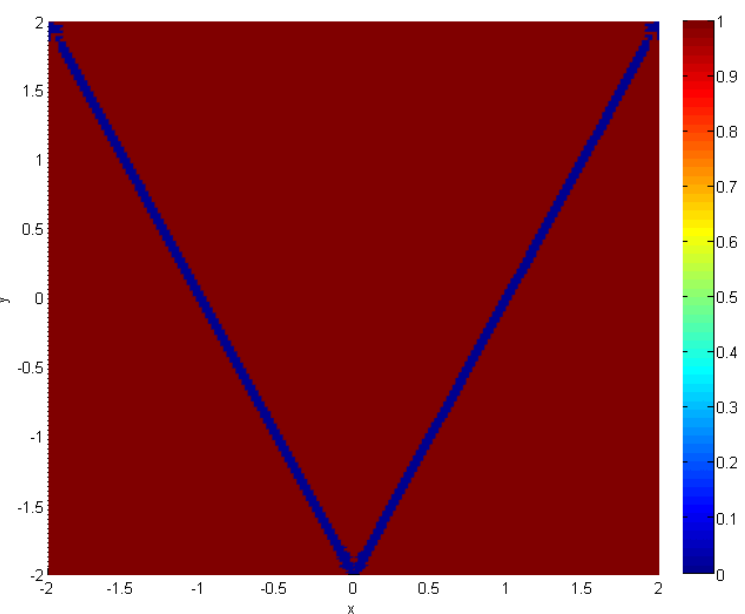

(b) Detector $-\sigma_{x}$

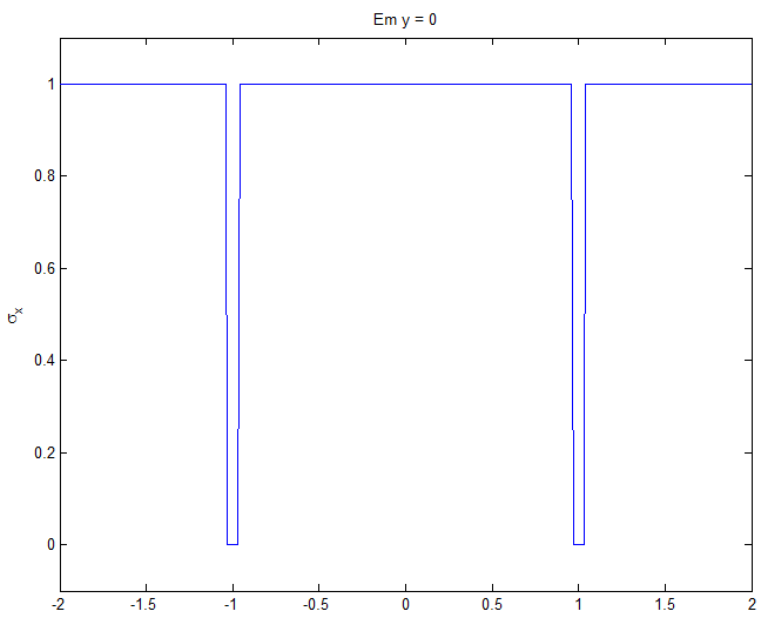

(d) Detector $-\sigma_{x}$ - Perfil em $y=0$

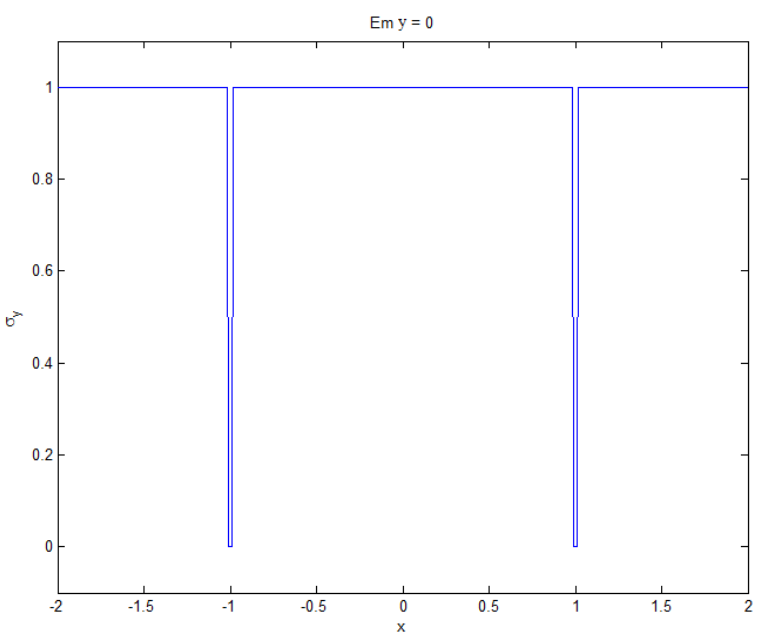

(e) Detector $-\sigma_{y}$ - Perfil em $y=0$

Figura 4.21: Novo detector 2D - Triângulo - $N_{x}=401$ e $N_{y}=401$ 


\subsection{Equações de Euler - 2D}

Com o novo detector de descontinuidades desenvolvido e os diversos testes realizados para funções bidimensionais, é possível aplicar o método numérico híbrido para resolver as equações de Euler 2D. Através da solução dessas equações, foram construídos gráficos que permitem a visualização e comparação da solução numérica do método híbrido com a solução obtida apenas através do método WENO.

As equações de Euler 2D na forma vetorial são dadas por

$$
\frac{\partial \mathbf{U}}{\partial t}+\frac{\partial \mathbf{F}}{\partial x}+\frac{\partial \mathbf{G}}{\partial y}=\mathbf{0}
$$

onde

$$
\mathbf{U}=\left[\begin{array}{c}
\rho \\
\rho u_{x} \\
\rho u_{y} \\
\rho e_{T}
\end{array}\right], \quad \mathbf{F}=\left[\begin{array}{c}
\rho u_{x} \\
p+\rho u_{x}^{2} \\
\rho u_{x} u_{y} \\
u_{x}\left(\rho e_{T}+p\right)
\end{array}\right], \quad \mathbf{G}=\left[\begin{array}{c}
\rho u_{y} \\
\rho u_{x} u_{y} \\
p+\rho u_{y}^{2} \\
u_{y}\left(\rho e_{T}+p\right)
\end{array}\right]
$$

Nota-se pelos vetores apresentados na equação (4.13) que há quatro equações e cinco incógnitas: densidade $(\rho)$, velocidade na direção $x\left(u_{x}\right)$, velocidade na direção $y\left(u_{y}\right)$, pressão $(p)$ e energia específica total $\left(e_{T}\right)$. Assim, é necessário construir uma relação entre duas incógnitas que permite resolver as equações de Euler, sendo

$$
p=(\gamma-1)\left[\rho e_{T}-\frac{\rho}{2}\left(u_{x}^{2}+u_{y}^{2}\right)\right]
$$

Para resolver as derivadas espaciais são utilizados os métodos híbrido e WENO; já para resolver a derivada temporal utiliza-se o método Runge-Kutta TVD de ordem 3 apresentado em [13]. Além disso, com a finalidade de resolver as equações de Euler numericamente, realiza-se a separação de fluxo apresentada em [28]. As equações de Euler para o caso bidimensional são detalhadas no apêndice B.

Com as equações de Euler para o caso bidimensional, é possível resolvê-las numericamente para uma determinada condição inicial. Com os resultados obtidos, são construídos gráficos que permitem comparar a solução do método híbrido com o WENO. Isso permite avaliar o desempenho de cada método e analisar as principais vantagens e desvantagens em aplicar cada um.

As condições iniciais consideradas para as equações de Euler 2D são:

- Circunferência:

$$
\left(\rho, u_{x}, u_{y}, p\right)= \begin{cases}(1.0,0.0,0.0,1.0), & \text { se } x^{2}+y^{2} \leq 0.5 \\ (0.125,0.0,0.0,1.0), & \text { caso contrário }\end{cases}
$$

$$
x \in[-2,2] \text { e } y \in[-2,2] .
$$


- Choque Incidente:

$$
\left(\rho, u_{x}, u_{y}, p\right)= \begin{cases}(1.0,2.9,0.0,0.71428), & \text { se } x \leq 0.0 \text { e } y \geq 1.0 \\ (1.69997,2.61934,-0.50632,1.52819), & \text { caso contrário }\end{cases}
$$

Para realizar a implementação das equações de Euler, foram consideradas as variáveis $N_{x}$ e $N_{y}$ que representam o número de pontos utilizados para a discretização na direção $x$ e $y$, respectivamente.

A Figura 4.22 mostra a condição inicial Circunferência (densidade $\rho$ e pressão $p$ ) utilizada para resolver numericamente as equações de Euler bidimensional.

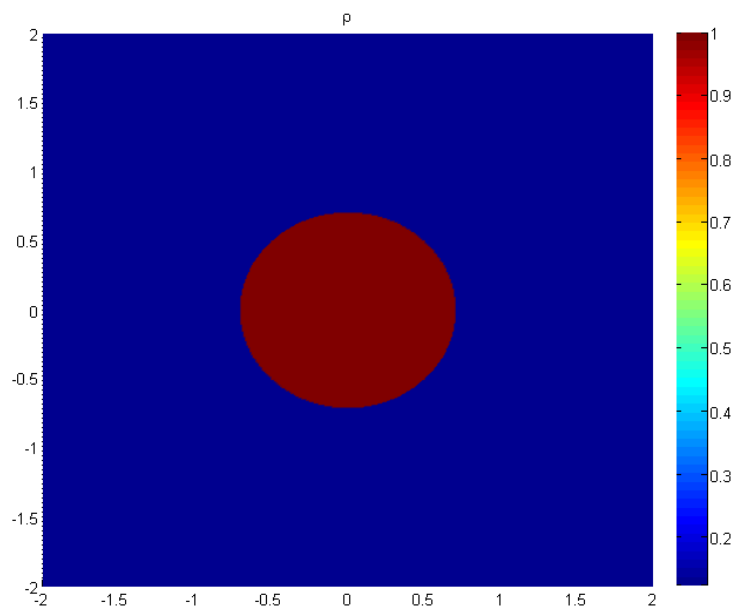

(a) Densidade

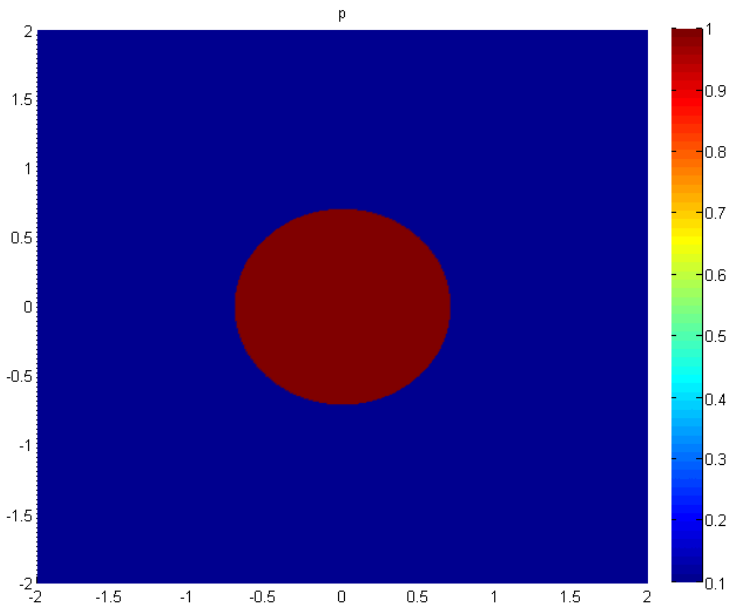

(b) Pressão

Figura 4.22: Euler 2D - Condição Inicial Circunferência - $T=0$

A Figura 4.23 apresenta os resultados obtidos para a densidade $(\rho)$, pressão $(p)$ e velocidades na direção $x$ e $y$ utilizando apenas o método WENO para a solução numérica das equações de Euler. Foi considerado $N_{x}=101$ e $N_{y}=101, C F L=0.05$ e tempo final $T=0.5$.

A fim de avaliar a solução obtida, a Figura 4.24 mostra os perfis em $y=0$ da densidade e pressão, permitindo assim visualizar o desempenho do método e as variações existentes no resultado.

Para comparar com o método WENO, as equações de Euler são resolvidas através do método híbrido com o novo detector desenvolvido para as mesmas configurações, ou seja, $N_{x}=101$, $N_{y}=101, C F L=0.05$ e tempo final $T=0.5$.

Observa-se pela Figura 4.25 que o resultado obtido é muito similar ao calculado somente com o método WENO. Assim, para realizar a comparação e avaliar com detalhes o desempenho de cada método, são contruídos os perfis em $y=0$. A Figura 4.26 mostra os perfis da densidade e pressão. 


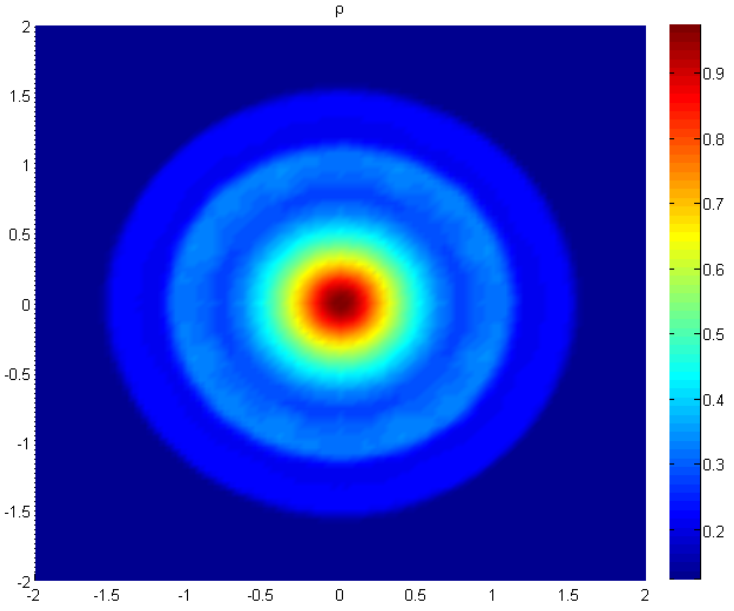

(a) Densidade

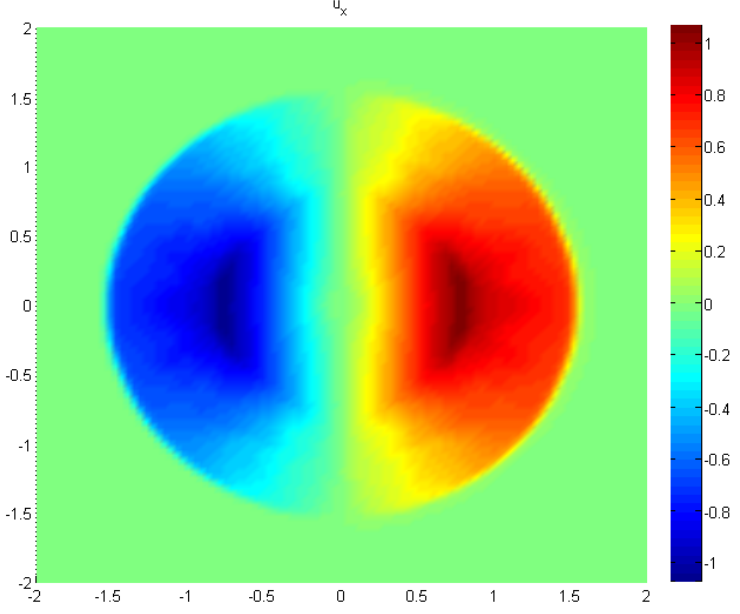

(c) Velocidade - Direção $x$

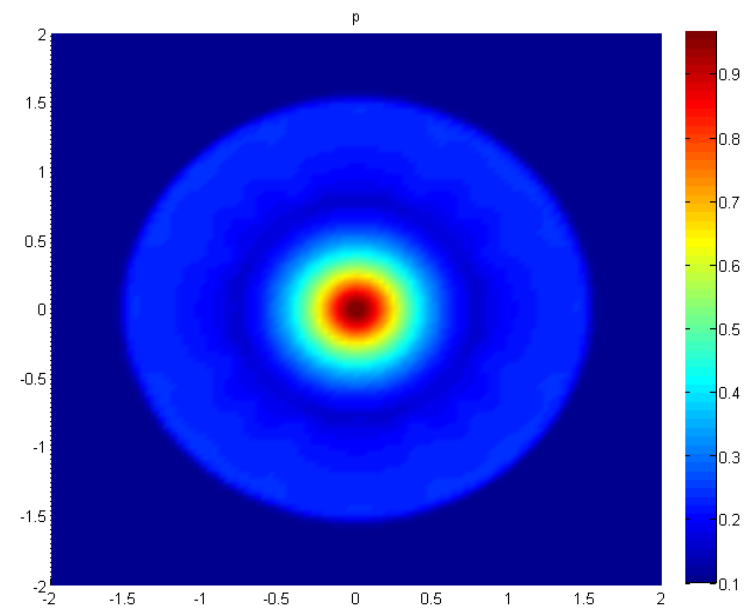

(b) Pressão

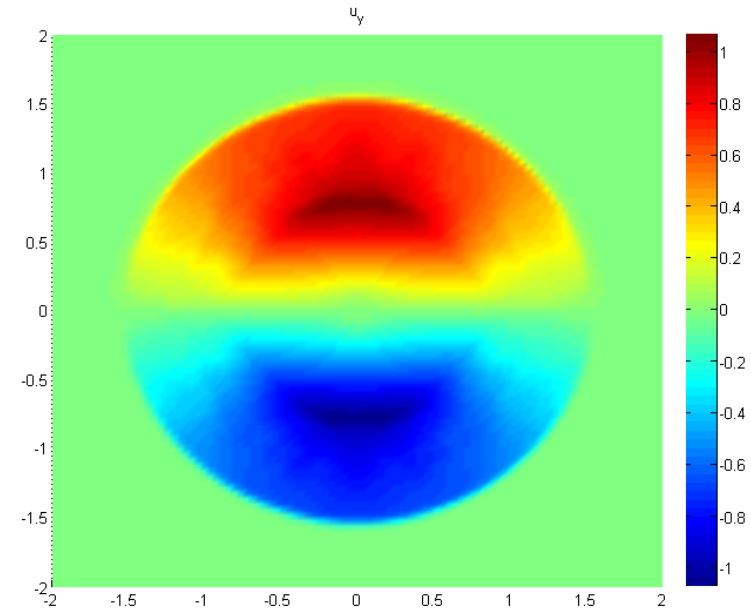

(d) Velocidade - Direção $y$

Figura 4.23: Euler 2D - WENO - $N_{x}=N_{y}=101, C F L=0.05$ e $T=0.5$ - Circunferência

Quando os métodos híbrido e WENO são comparados, é possível notar uma diferença no resultado calculado numericamente. Sendo assim, na Figura 4.27 compara-se em um mesmo gráfico os perfis calculados com o método WENO e com o método híbrido. Observa-se, pelas Figuras 4.27(a) e 4.27(b), que o resultado obtido pelo método numérico híbrido apresenta mais detalhes quando comparado com o método WENO.

Com a intenção de comparar o resultado para uma malha com mais pontos na direção $x$ e $y$, foi considerado o caso com $N_{x}=401$ e $N_{y}=401, C F L=0.05$ e tempo final $T=0.5$.

Com os perfis em $y=0$ da densidade e pressão, é possível observar pela Figura 4.28 que o resultado calculado apresenta algumas diferenças quando comparado com o mesmo caso e menos pontos na discretização (Figura 4.24).

A Figura 4.29 mostra os perfis da densidade e pressão em $y=0$ para o método híbrido.

Para comparar as soluções calculadas através do método WENO e do método híbrido, a Figura 4.30 mostra os perfis em $y=0$. 


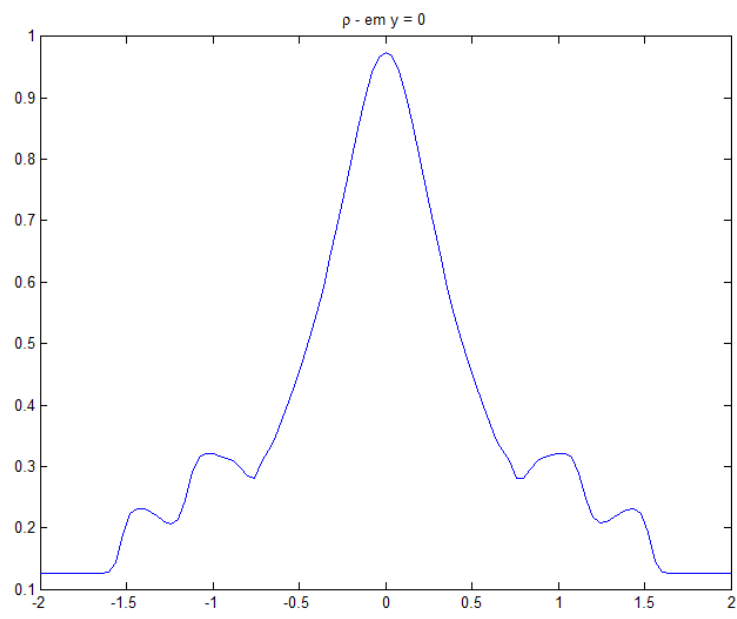

(a) Densidade

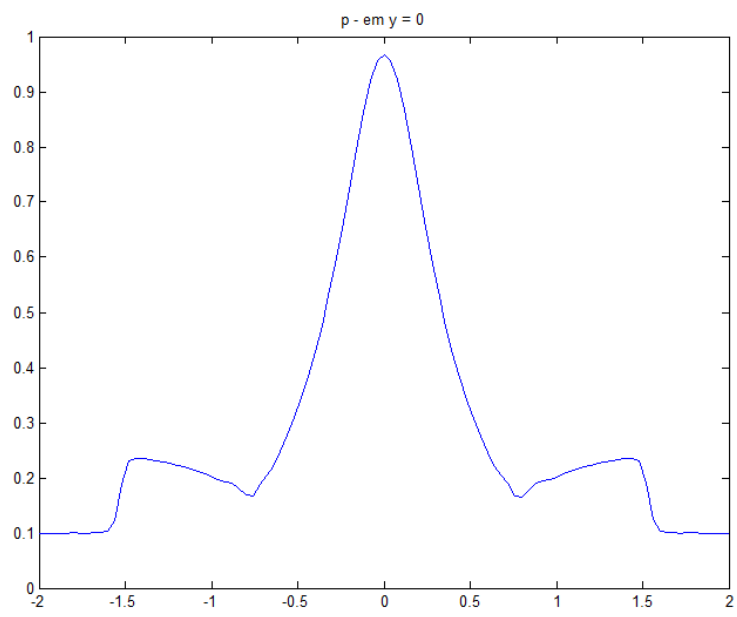

(b) Pressão

Figura 4.24: Euler 2D - WENO - Perfil em $y=0-N_{x}=N_{y}=101, C F L=0.05$ e $T=0.5$ Circunferência

Pelas Figuras 4.30(a) e 4.30(b) é possível observar que na região [-0.5, 0.5] o método WENO apresenta dispersão, ou seja, perda de informações da solução numérica calculada. Com isso, a fim de comparar e avaliar, resolve-se numeriacmente as equações de Euler através do método híbrido.

Nota-se que, assim como para o caso com menos pontos na discretização, o método numérico híbrido apresenta uma solução com mais detalhes, como pode ser observado nas Figuras 4.30(a) e 4.30(b).

A Tabela 4.5 mostra os tempos computacionais consumidos com os métodos WENO e híbrido para a solução das equações de Euler bidimensionais com a condição inicial Circunferência.

\begin{tabular}{|l|l|l|}
\cline { 2 - 3 } \multicolumn{1}{c|}{} & \multicolumn{2}{c|}{ Tempo } \\
\hline$N_{x} \times N_{y}$ & \multicolumn{1}{c|}{ WENO } & \multicolumn{1}{c|}{ Híbrido } \\
\hline $81 \times 81$ & $1 m 6.11 s$ & $1 m 7.10 s$ \\
\hline $101 \times 101$ & $2 m 2.74 s$ & $2 m 2.79 s$ \\
\hline $201 \times 201$ & $17 m 17.4 s$ & $20 m 18.7 s$ \\
\hline $401 \times 401$ & $140 m 51.9 s$ & $142 m 2.69 s$ \\
\hline
\end{tabular}

Tabela 4.5: Tabela de tempos de execução - Euler 2D - Circunferência

Observa-se que, assim como para o caso unidimensional, o tempo consumido quando é utilizado o método híbrido é maior do que o método WENO. Porém, o resultado obtido com o método híbrido apresenta mais detalhes na solução numérica, sendo então necessário menos pontos na discretização quando comparado com o método WENO.

A Figura 4.31 mostra os resultados obtidos para a densidade $(\rho)$, pressão $(p)$ e velocidades nas direção $x$ e $y$, calculados através do método WENO para a solução numérica das equações de Euler com a condição inicial Choque Incidente. São considerados $N_{x}=83, N_{y}=31, C F L=0.3$ e tempo final $T=5.0$. 


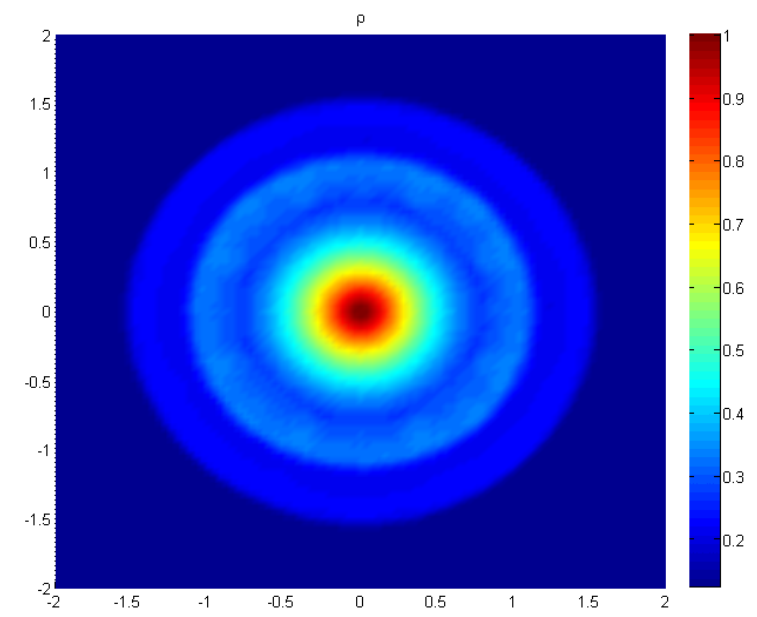

(a) Densidade

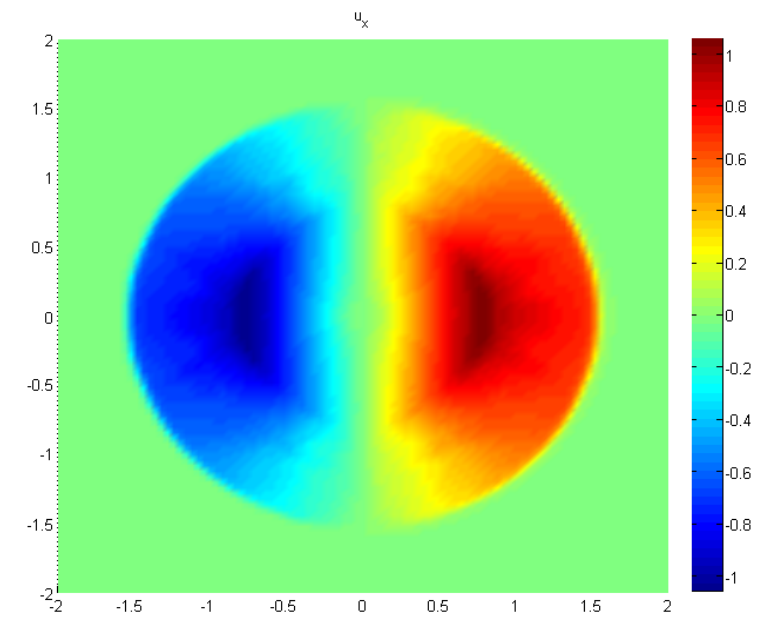

(c) Velocidade - Direção $x$

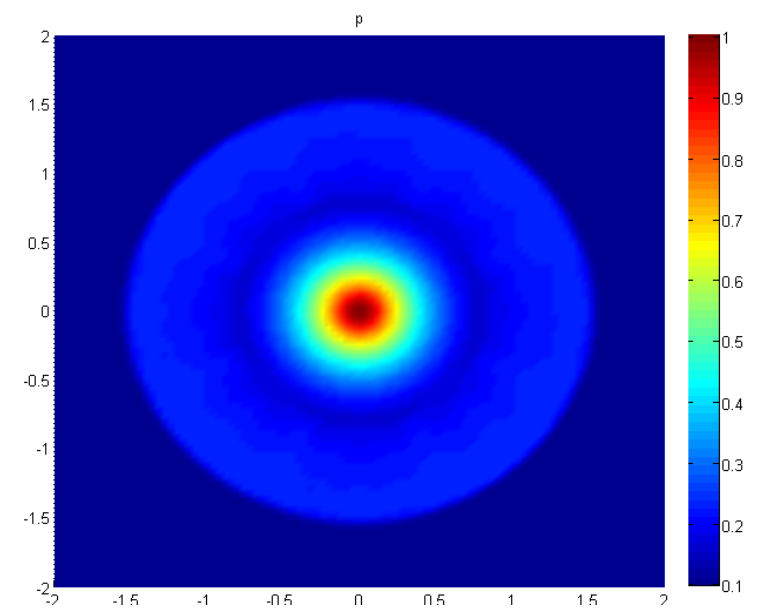

(b) Pressão

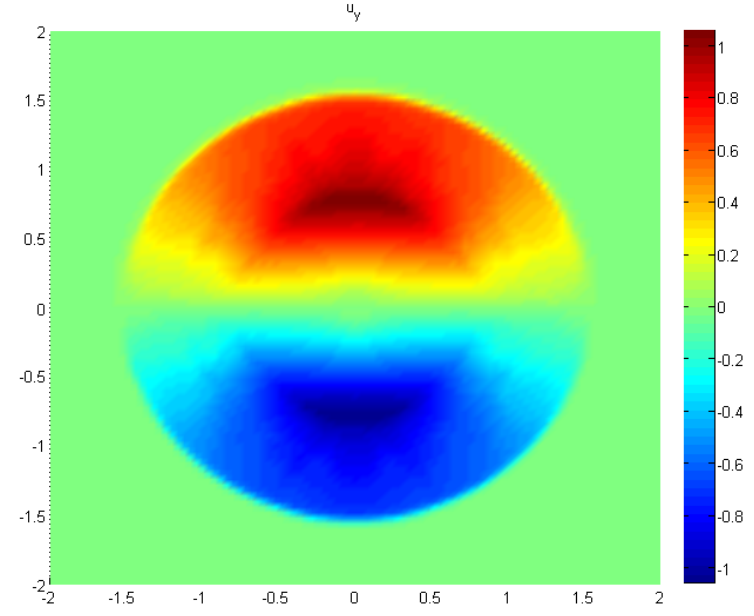

(d) Velocidade - Direção $y$

Figura 4.25: Euler 2D - Híbrido - $N_{x}=N_{y}=101, C F L=0.05$ e $T=0.5$ - Circunferência

Com o objetivo de avaliar a solução numérica obtida, a Figura 4.32 mostra os perfis da densidade e pressão em $y=0.75$, permitindo observar o desempenho do método e as variações existentes no resultado.

Para comparar com o método WENO, as equações de Euler para a condição inicial Choque Incidente são resolvidas através do método numérico híbrido, com as mesmas configurações.

Nota-se pela Figura 4.33 que o resultado é muito similiar ao calculado pelo método WENO, sendo então necessária a construção dos perfis em $y=0.75$ para comparar os métodos.

Dessa forma, a Figura 4.34 mostra os perfis para a densidade $(\rho)$ e pressão $(p)$ em $y=0.75$ determinados com o método híbrido.

A Figura 4.35 mostra uma comparação entre os métodos WENO e híbrido através dos perfis de densidade e pressão em $y=0.75$.

Observa-se pelas Figuras 4.35(a) e 4.35(b) que os resultados obtidos são similares.

A fim de comparar o resultado, a mesma condição inicial foi considerada para uma malha com mais pontos na direção $x$ e $y$, sendo $N_{x}=165, N_{y}=61, C F L=0.3$ e tempor final $T=5.0$. 


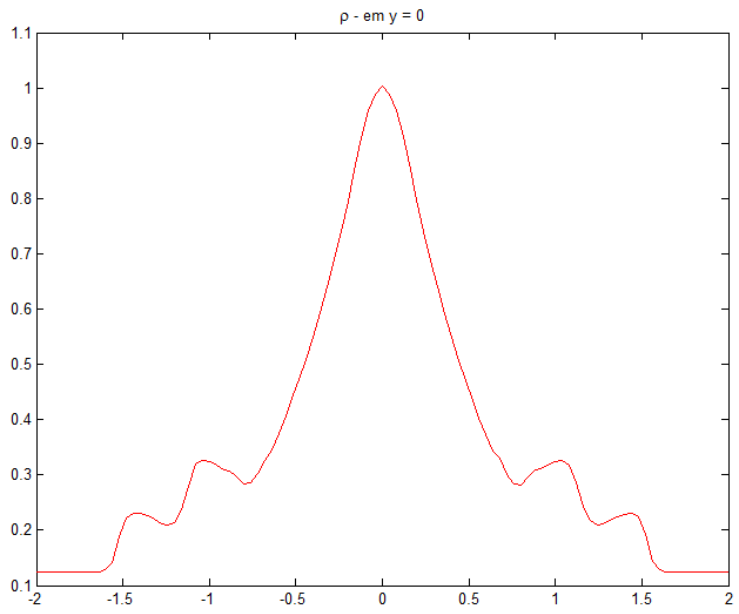

(a) Densidade

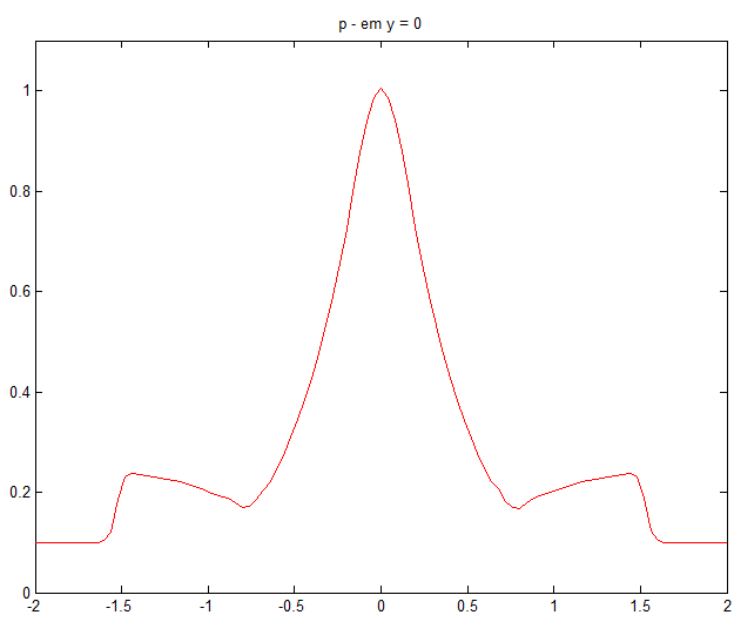

(b) Pressão

Figura 4.26: Euler 2D - Híbrido - Perfil em $y=0-N_{x}=N_{y}=101, C F L=0.05$ e $T=0.5$ Circunferência

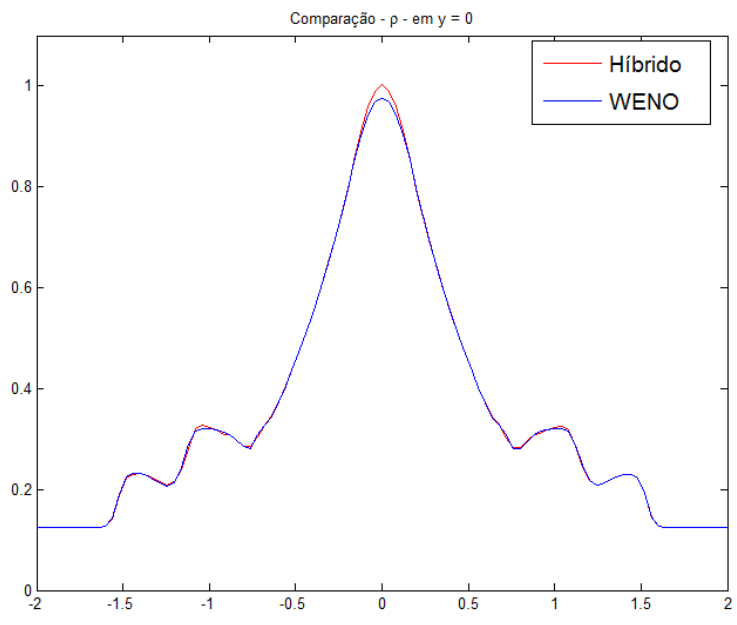

(a) Densidade

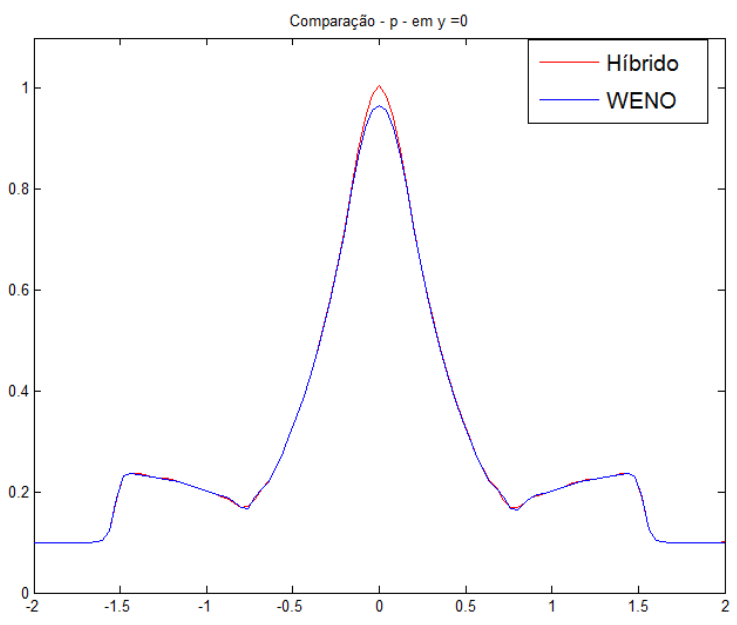

(b) Pressão

Figura 4.27: Euler 2D - Comparação - Perfil em $y=0-N_{x}=N_{y}=101, C F L=0.05$ e $T=0.5$ - Circunferência

A Figura 4.36 mostra o resultado obtido para as equações de Euler com o método WENO.

Com os perfis de densidade e pressão em $y=0.75$ apresentados na Figura 4.37 é possível obervar algumas mudanças em relação aos resultados apresentados na Figura 4.32.

Já a Figura 4.38 mostra os resultados para a densidade $(\rho)$, pressão $(p)$ e velocidades nas direções $x$ e $y$ calculados com o método híbrido para $N_{x}=165, N_{y}=61, C F L=0.3$ e tempo final $T=5.0$.

Assim como no caso do método WENO, os perfis de densidade e pressão para $y=0.75$ (Figura 4.39) apresentam algumas diferenças quando comparados com a malha com menos pontos na discretização (Figura 4.34). 


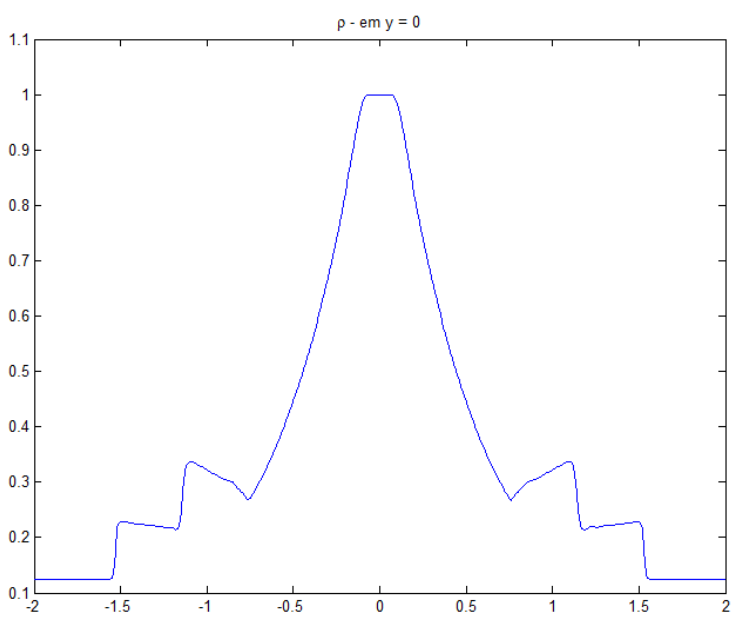

(a) Densidade

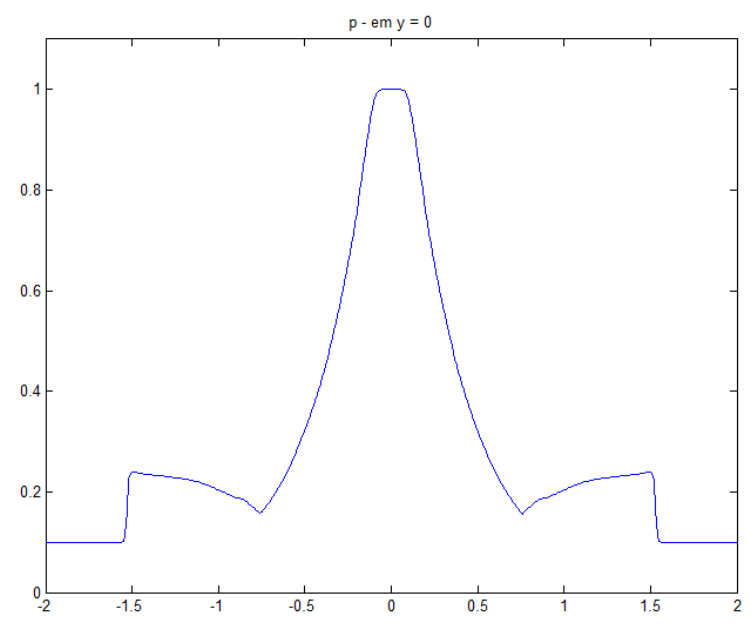

(b) Pressão

Figura 4.28: Euler 2D - WENO - Perfil em $y=0$ - $N_{x}=N_{y}=401, C F L=0.05$ e $T=0.5$ Circunferência

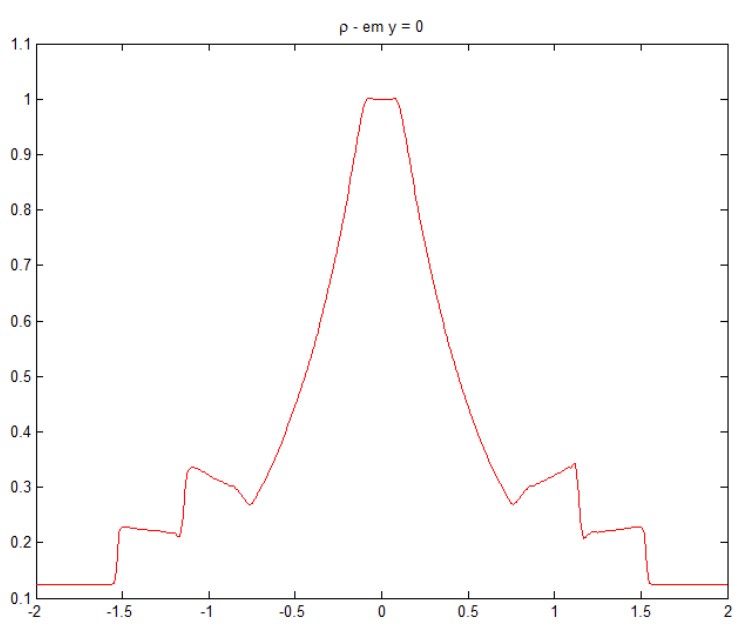

(a) Densidade

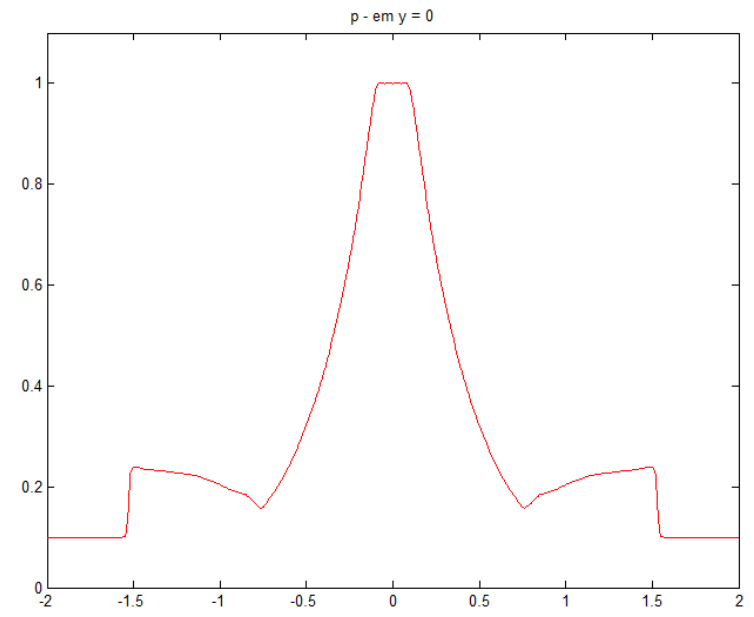

(b) Pressão

Figura 4.29: Euler 2D - Híbrido - Perfil em $y=0$ - $N_{x}=N_{y}=401, C F L=0.05$ e $T=0.5$ Circunferência

Com isso, através da comparação dos perfis de densidade e pressão obtidos com os método WENO e híbrido, é possível observar na Figura 4.40 que os resulatdos são similares e apresentam pequenas diferenças.

A Tabela 4.6 mostra os tempos computacionais consumidos com os métodos WENO e híbrido para a solução das equações de Euler bidimensionais com a condição inicial Choque Incidente. 


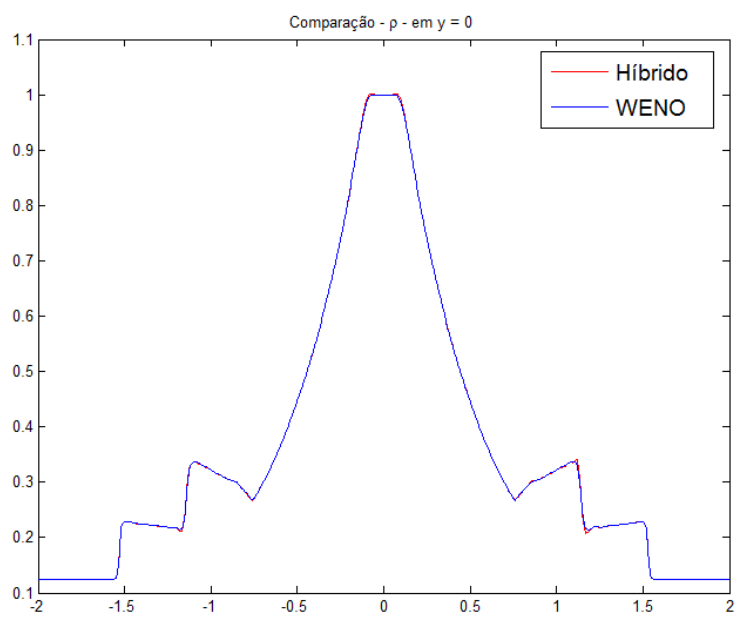

(a) Densidade

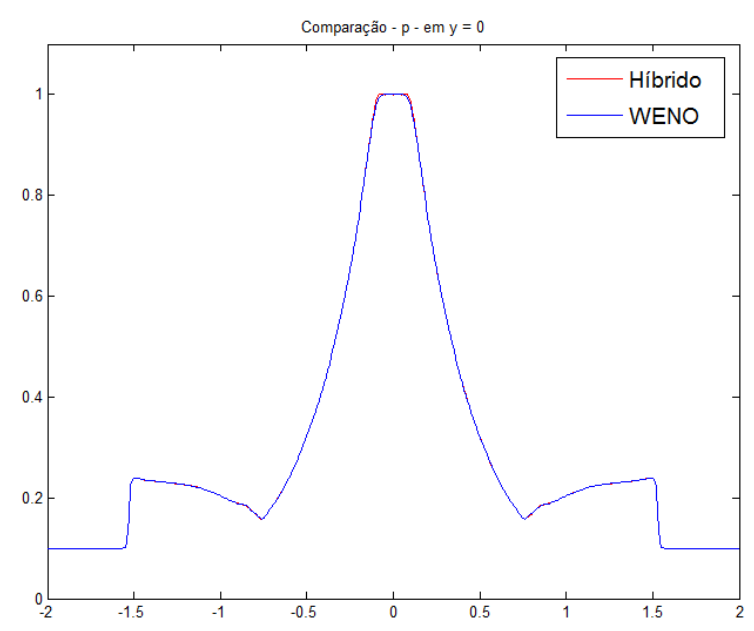

(b) Pressão

Figura 4.30: Euler 2D - Comparação - Perfil em $y=0-N_{x}=N_{y}=401, C F L=0.05$ e $T=0.5$ - Circunferência

\begin{tabular}{|l|l|l|}
\cline { 2 - 3 } \multicolumn{1}{c|}{} & \multicolumn{2}{c|}{ Tempo } \\
\hline$N_{x} \times N_{y}$ & \multicolumn{1}{c|}{ WENO } & \multicolumn{1}{c|}{ Híbrido } \\
\hline $83 \times 31$ & $3 m 90.4 s$ & $4 m 17.4 s$ \\
\hline $165 \times 61$ & $15 m 79.89 s$ & $16 m 97.08 s$ \\
\hline $321 \times 121$ & $360 m 25.2 s$ & $362 m 87.5 s$ \\
\hline
\end{tabular}

Tabela 4.6: Tabela de tempos de execução - Euler 2D - Choque Incidente

Observa-se que, assim como para o caso unidimensional e bidimensional com a condição inicial Circunferência, o tempo consumido quando é utilizado o método híbrido é maior do que o método WENO. Porém, o método híbrido obtém a mesma qualidade de solução do método WENO com uma malha com menos pontos.

\subsubsection{Observações}

Para resolver as equações de Euler bidimensionais, é necessário aplicar o detector de descontinuidades nas direções $x$ e $y$ e obter consequentemente os pesos $\sigma_{x}$ e $\sigma_{y}$. Assim, com os pesos determinados e as regiões definidas, o método híbrido desenvolvido pode ser aplicado para obter a solução numérica do problema. Dessa forma, foram consideradas diversas condições inicias que permitem avaliar o desempenho do método híbrido desenvolvido para diferentes discretizações e compará-lo com o método WENO, avaliando as principais vantagens e desvantagens de cada um.

Com o objetivo de resolver as equações de Euler para as condições iniciais Circunferência e Choque Incidente, foram consideradas condições de fronteira desenvolvidas em [22], [30] e [31] que permitem definir o domínio computacional corretamente. 


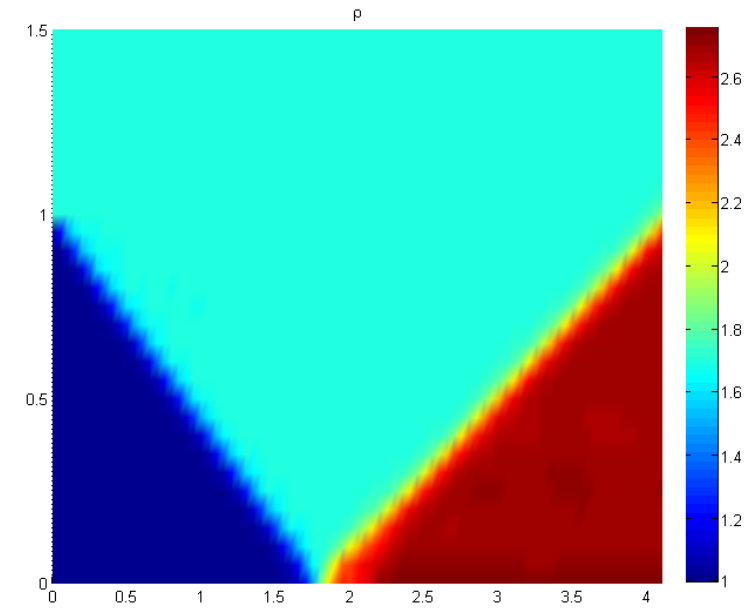

(a) Densidade

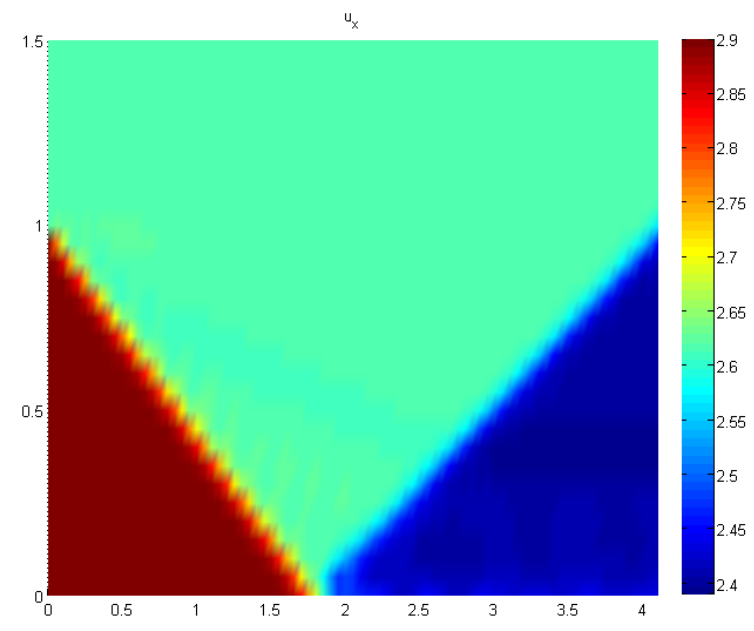

(c) Velocidade - Direção $x$

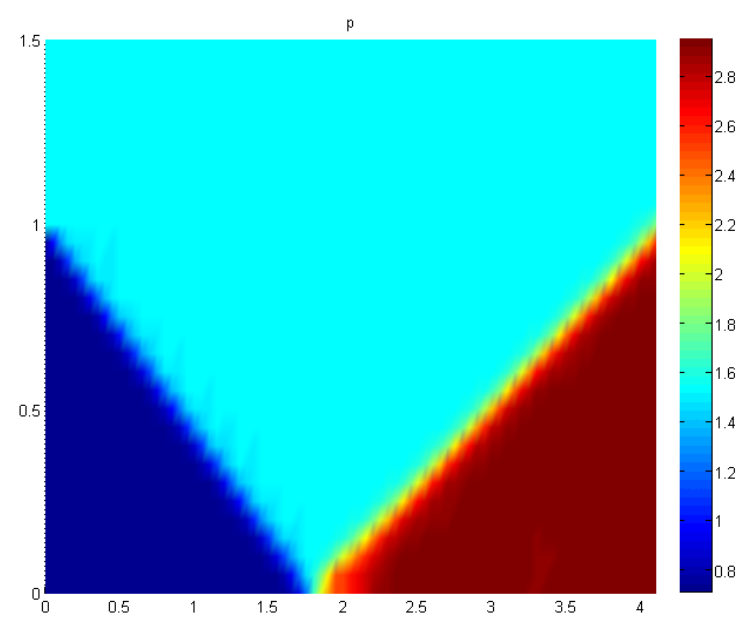

(b) Pressão

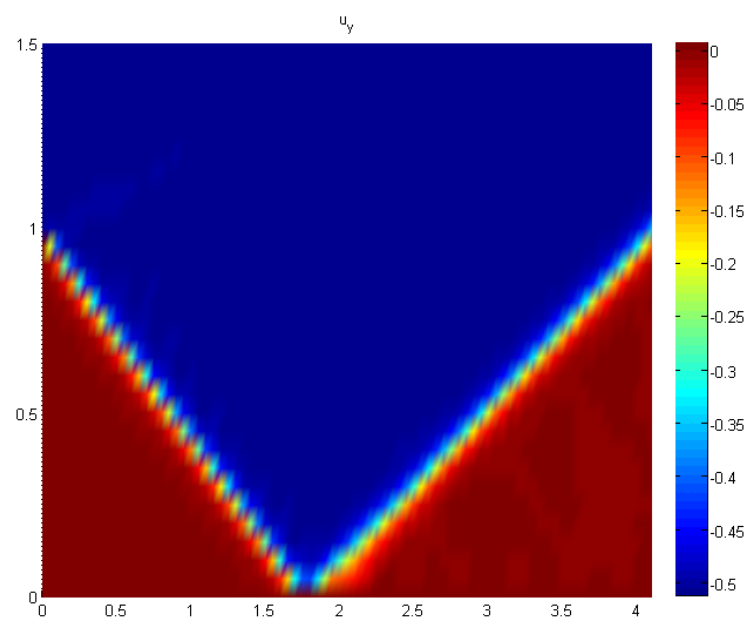

(d) Velocidade - Direção $y$

Figura 4.31: Euler 2D - WENO - $N_{x}=83$ e $N_{y}=31, C F L=0.3$ e $T=5.0$ - Choque Incidente

A condição inicial Circunferência é resolvida até o tempo final $T=0.5$, sendo que a solução final não chega até as fronteiras do domínio considerado. Dessa forma, é considerado que as mesmas estão distantes da descontinuidade.

Já para a condição inicial Choque Incidente, a solução numérica é calculada até o tempo final $T=5.0$, ou seja, até encontrar-se em estado estacionário. Pelo fato de existir uma descontinuidade e a mesma ser propagada em cada passo, é necessário que seja realizado um tratamento de fronteira específico, sendo que esse choque é refletido em uma das fronteiras do domínio, chamada de parede (slip wall). 


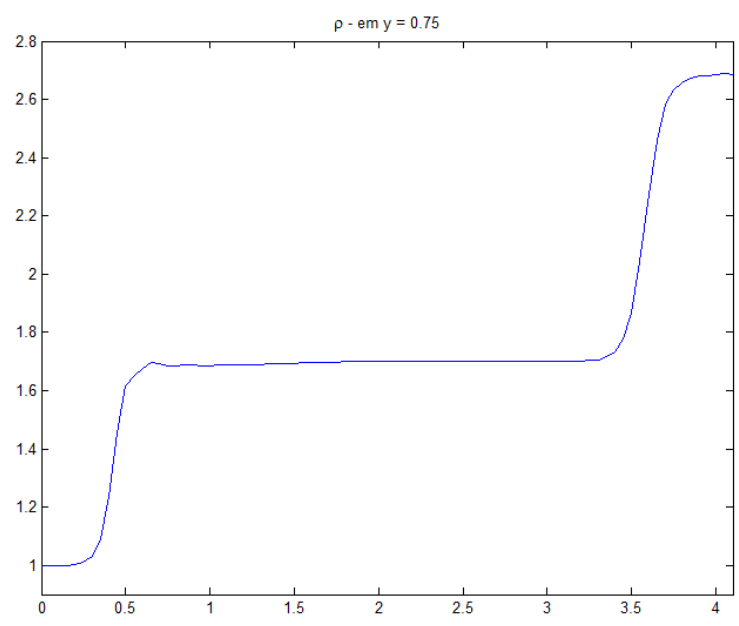

(a) Densidade

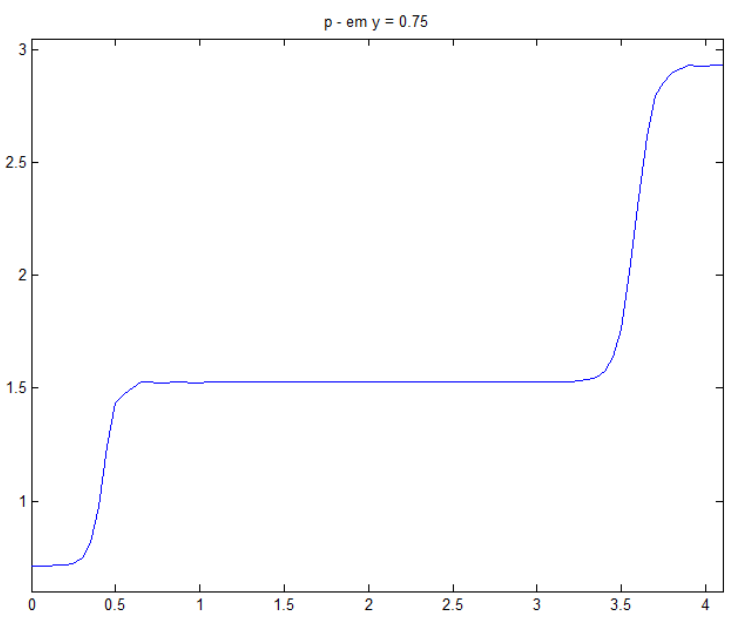

(b) Pressão

Figura 4.32: Euler 2D - WENO - Perfil em $y=0.75-N_{x}=83$ e $N_{y}=31, C F L=0.3$ e $T=5.0$ - Choque Incidente

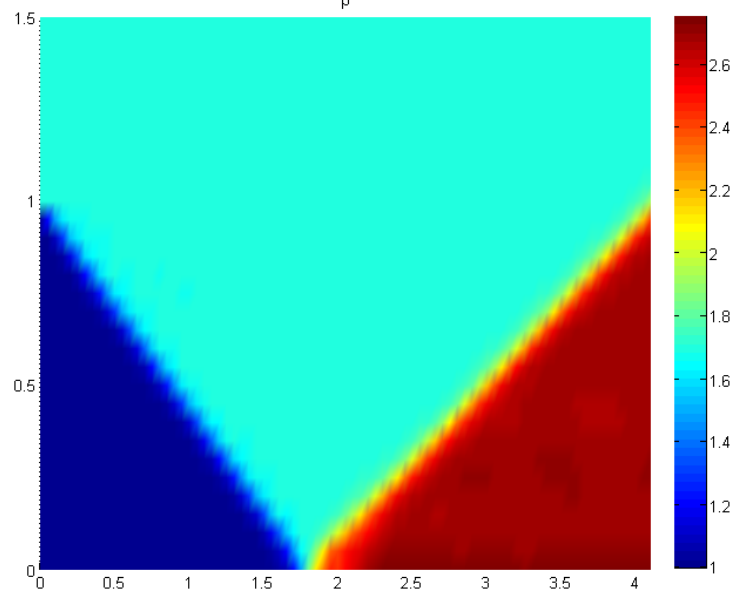

(a) Densidade

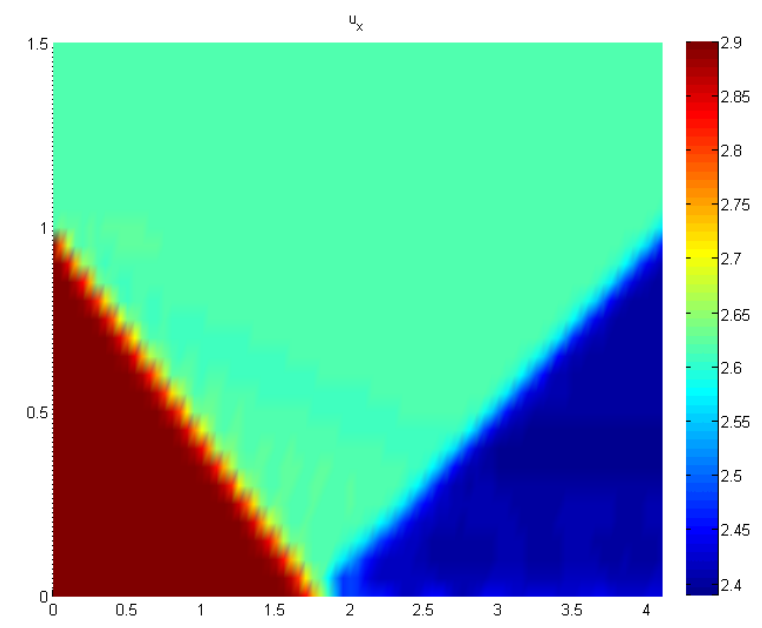

(c) Velocidade - Direção $x$

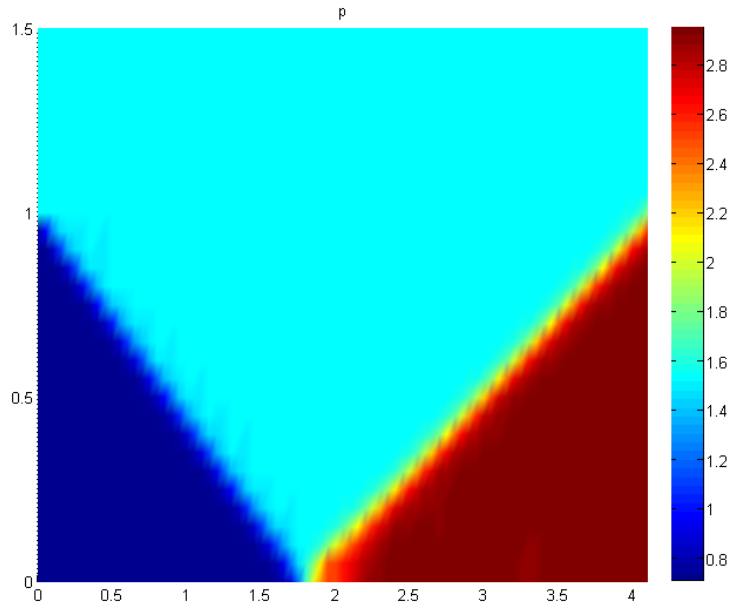

(b) Pressão

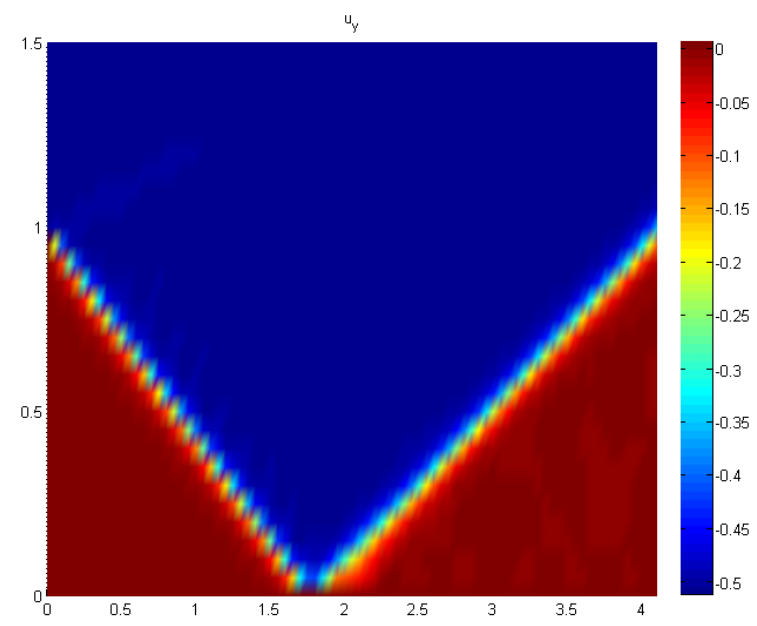

(d) Velocidade - Direção $y$

Figura 4.33: Euler 2D - Híbrido - $N_{x}=83$ e $N_{y}=31, C F L=0.3$ e $T=5.0$ - Choque Incidente 


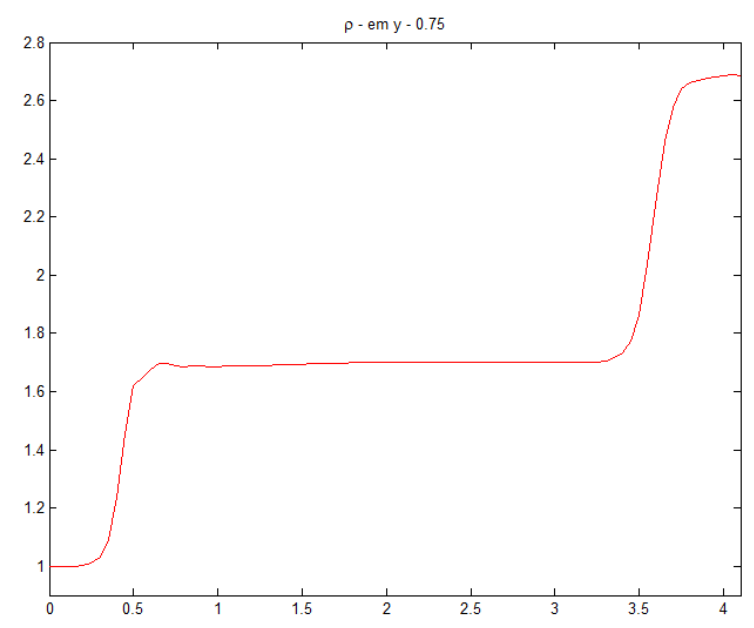

(a) Densidade

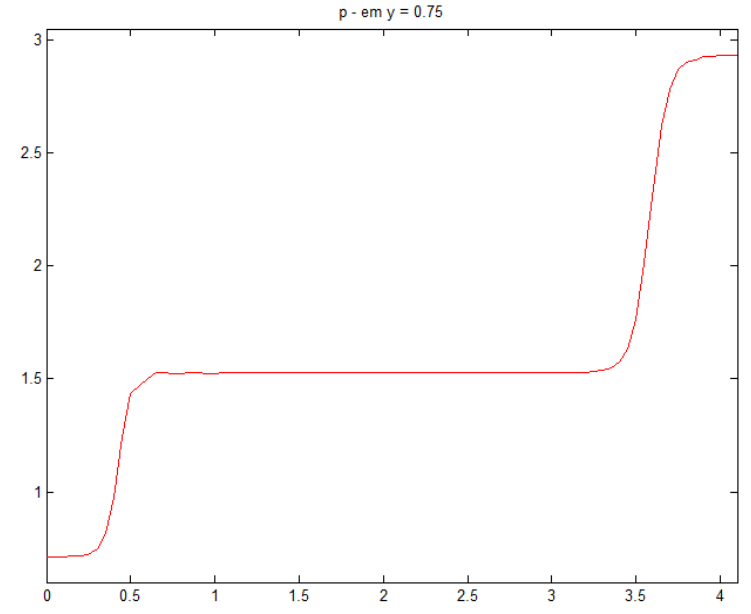

(b) Pressão

Figura 4.34: Euler 2D - Híbrido - Perfil em $y=0.75-N_{x}=83$ e $N_{y}=31, C F L=0.3$ e $T=5.0$ - Choque Incidente

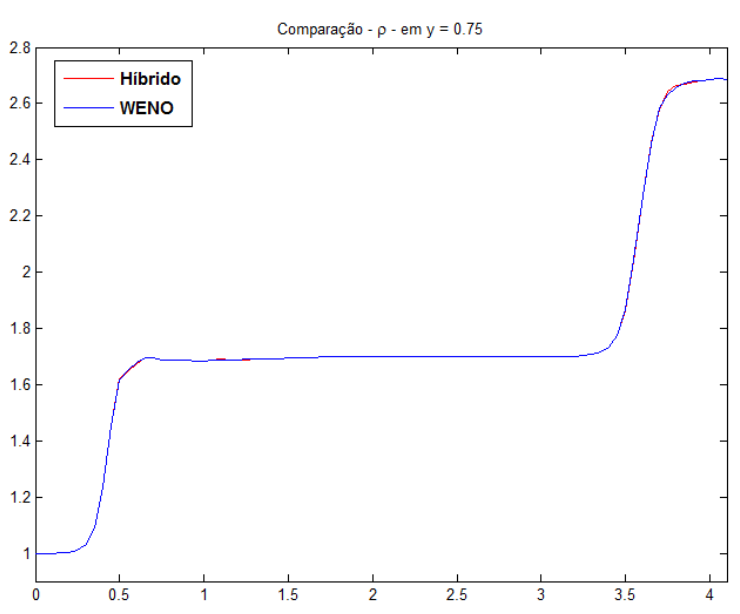

(a) Densidade

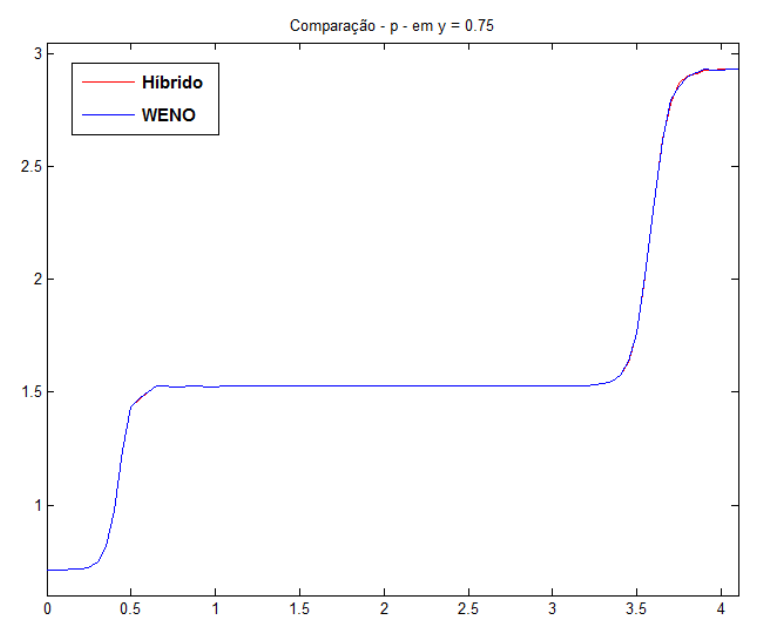

(b) Pressão

Figura 4.35: Euler 2D - Comparação - Perfil em $y=0.75-N_{x}=83$ e $N_{y}=31, C F L=0.3$ e $T=5.0$ - Choque Incidente 


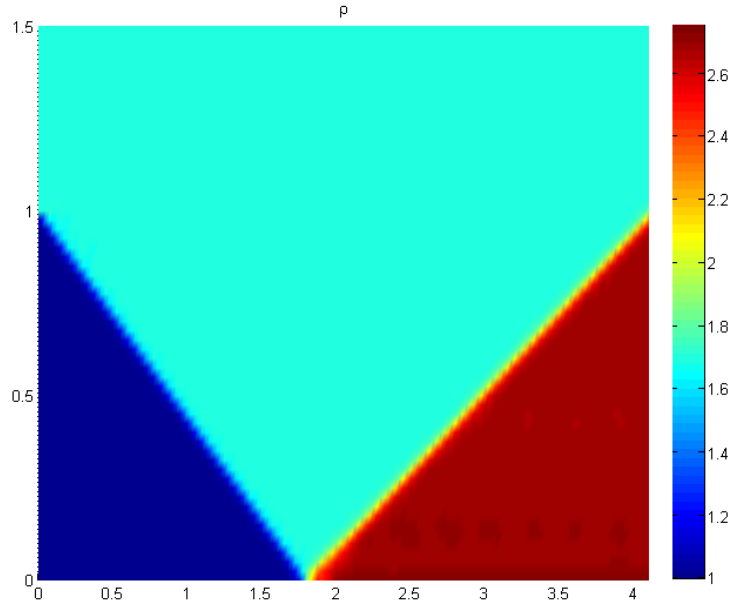

(a) Densidade

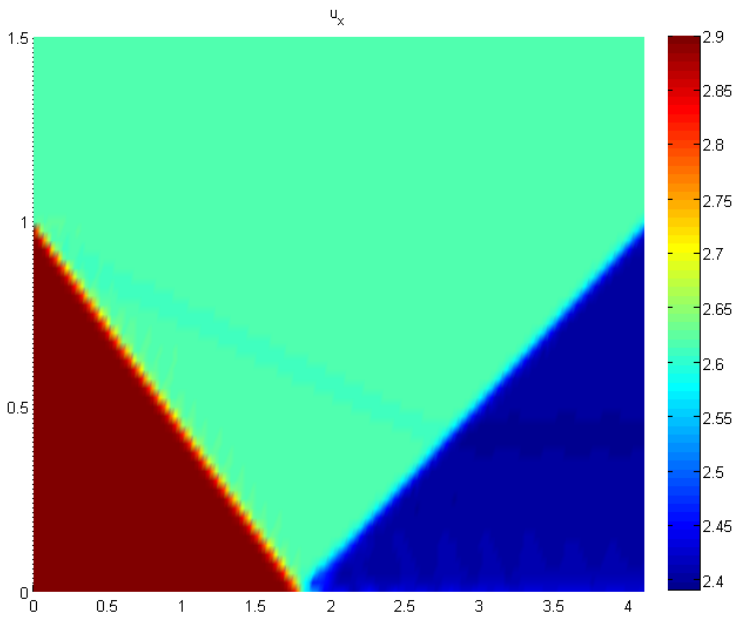

(c) Velocidade - Direção $x$

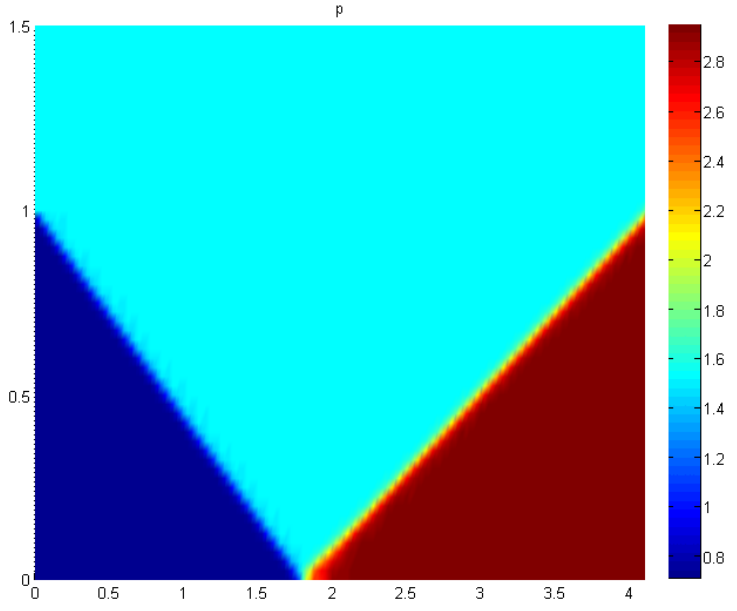

(b) Pressão

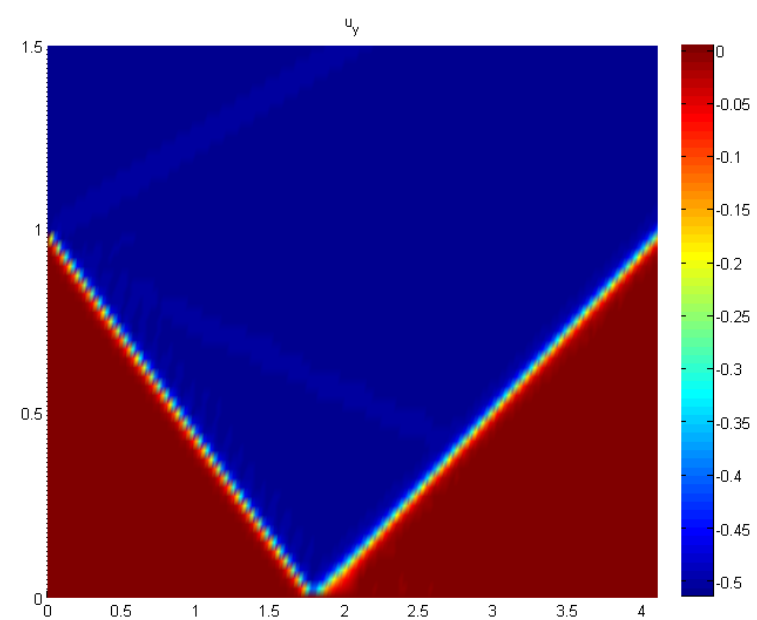

(d) Velocidade - Direção $y$

Figura 4.36: Euler 2D - WENO - $N_{x}=165$ e $N_{y}=61, C F L=0.3$ e $T=5.0$ - Choque Incidente

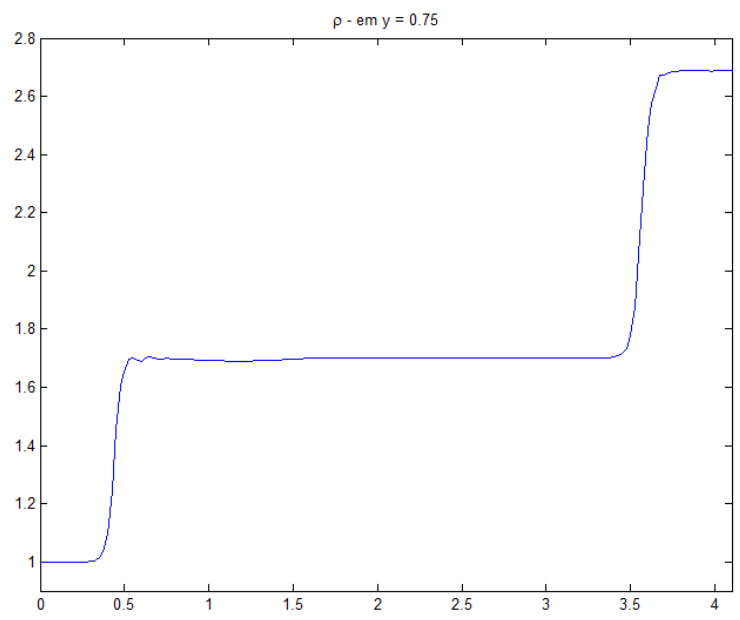

(a) Densidade

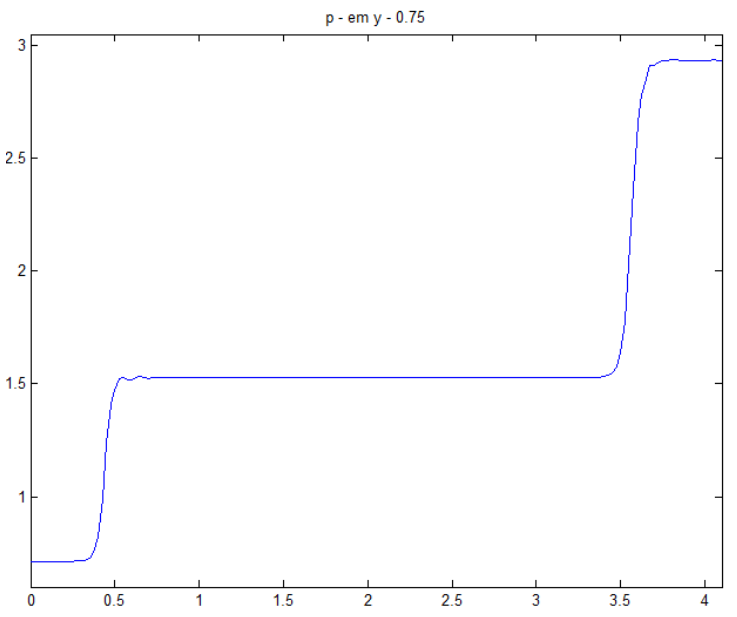

(b) Pressão

Figura 4.37: Euler 2D - WENO - Perfil em $y=0.75-N_{x}=165$ e $N_{y}=61, C F L=0.3$ e $T=5.0$ - Choque Incidente 


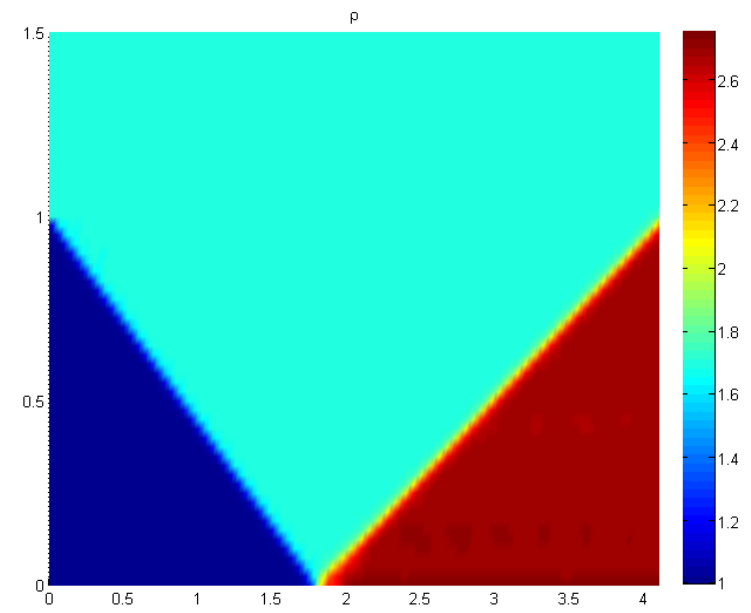

(a) Densidade

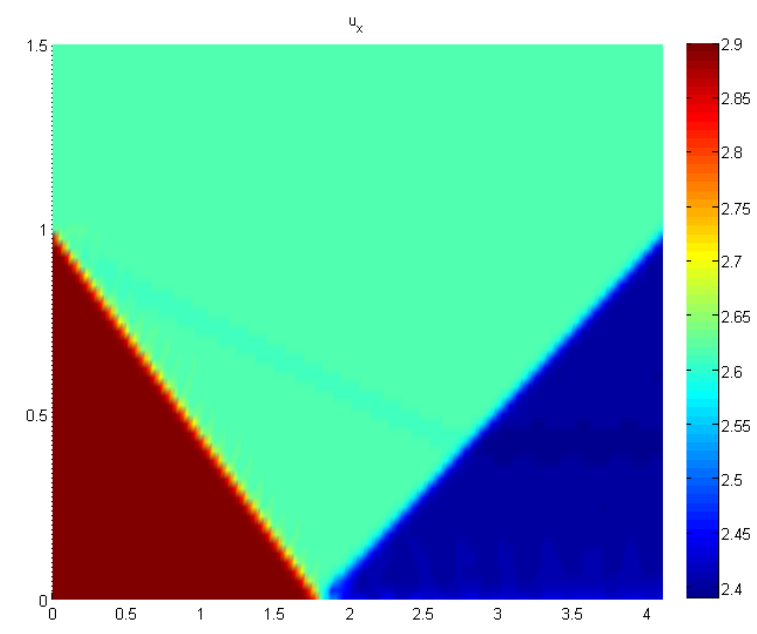

(c) Velocidade - Direção $x$

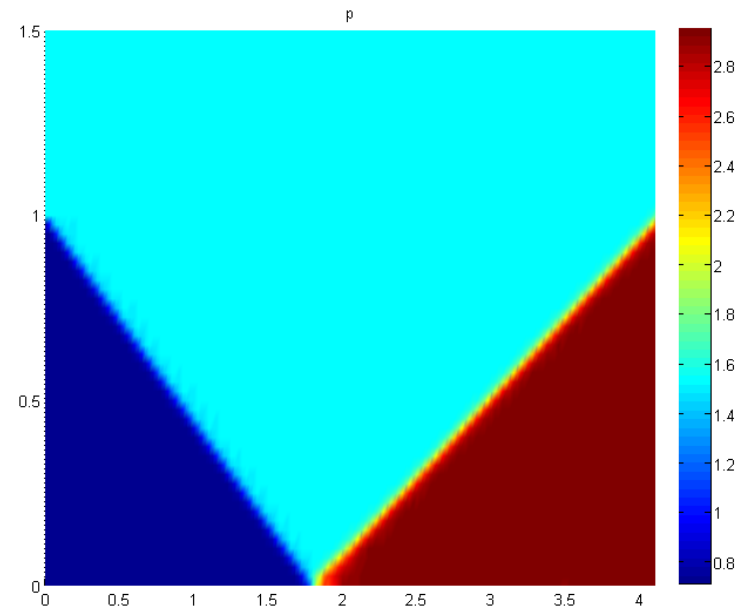

(b) Pressão

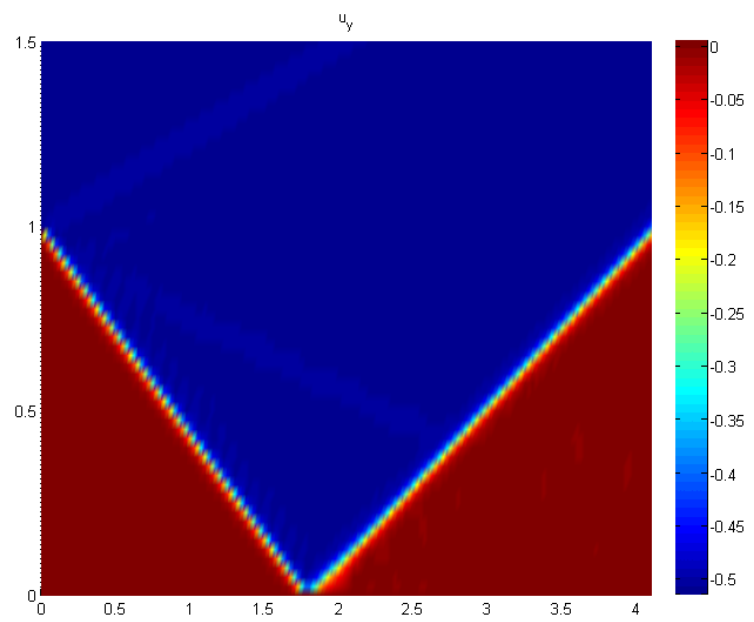

(d) Velocidade - Direção $y$

Figura 4.38: Euler 2D - Híbrido - $N_{x}=165$ e $N_{y}=61, C F L=0.3$ e $T=5.0$ - Choque Incidente

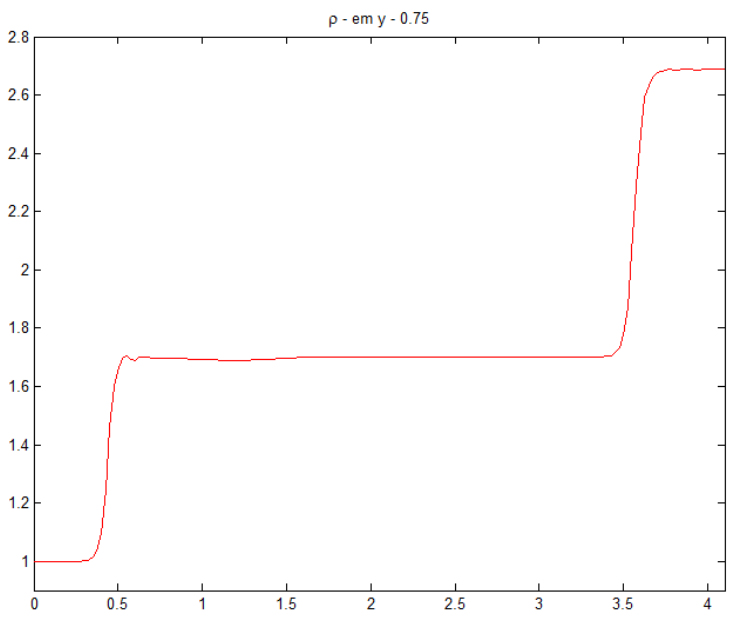

(a) Densidade

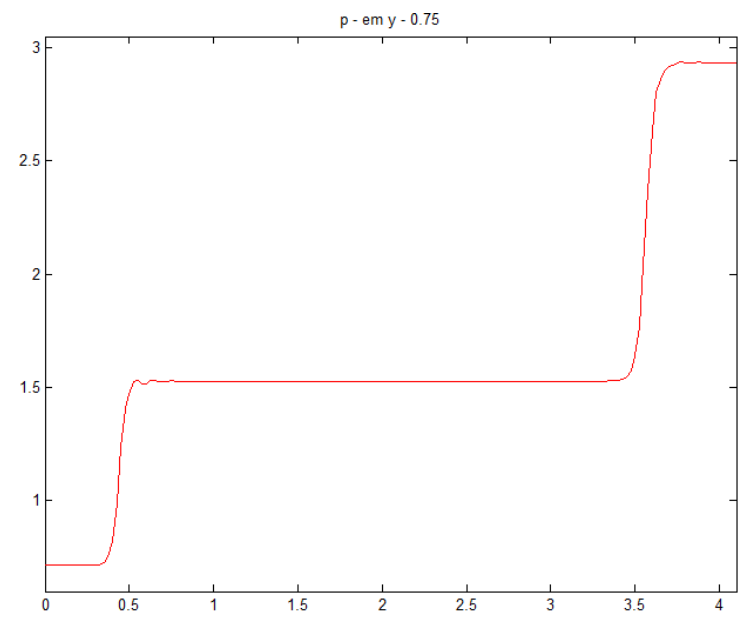

(b) Pressão

Figura 4.39: Euler 2D - Híbrido - Perfil em $y=0-N_{x}=165$ e $N_{y}=61, C F L=0.3$ e $T=5.0$ 


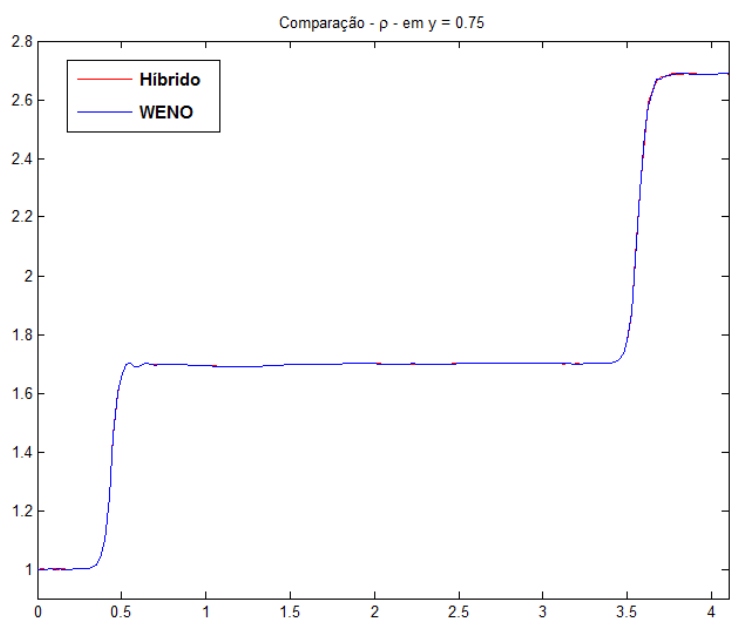

(a) Densidade

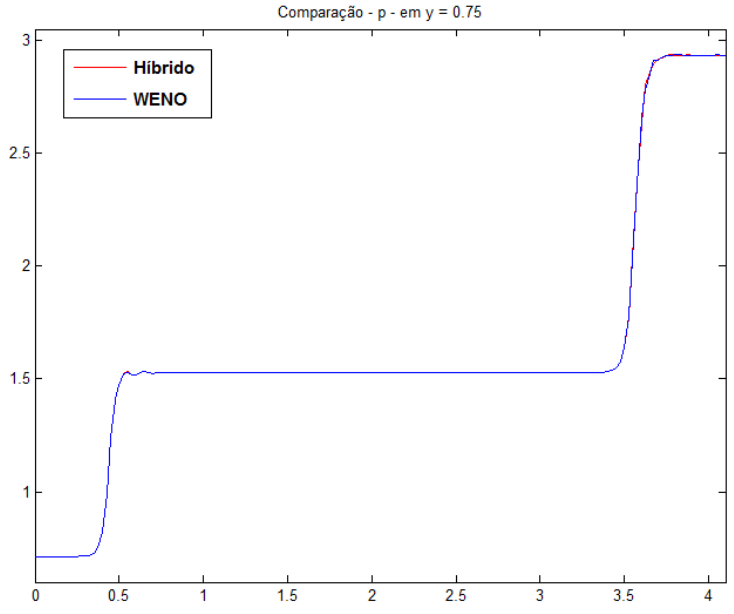

(b) Pressão

Figura 4.40: Euler 2D - Comparação - Perfil em $y=0$ - $N_{x}=165$ e $N_{y}=61, C F L=0.3$ e $T=5.0$ - Choque Incidente 

Com a revisão bibliográfica dos diversos métodos numéricos híbridos existentes e a avaliação dos detectores de descontinuidades para funções unidimensionais, foi possível avaliar as principais vantagens e desvantagens que cada um apresenta na determinação das regiões que possuem função suave ou descontínua.

Através dessas avaliações obtidas da análise dos métodos desenvolvidos por Zhou, Pirozzoli, Adams, Yee, Hill e Kim desenvolveu-se um novo detector de descontinuidades que utiliza o cálculo e combinação das derivadas de ordem 4, 5 e 6 para uma malha mais fina com espaçamento $h$ e uma malha mais grossa com espaçamento $2 h$. Com o método de diferenças finitas de ordem 6 para o cálculo das derivadas, a razão entre as malhas permitiu construir um indicador de suavidade cujo valor $2^{4}=16$ é obtido para regiões que apresentam função suave. Já para os casos em que a região apresenta descontinuidade foi observada a relação $2^{n}$.

Quando uma função apresenta descontinuidade na $n$-ésima derivada, o valor obtido para o indicador de suavidade nessa região é $2^{n}$, como foi observado nos diversos exemplos apresentados, sendo que estes apresentavam descontinuidades na própria função ou em suas derivadas. Além dos exemplos, essa relação foi demonstrada teoricamente.

Com a relação $2^{n}$ descrita, foi possível determinar o valor limiar para a construção do parâmetro $\sigma$ que indica de fato se uma região pode ser considerada com função suave ou descontínua, permitindo definir qual método numérico será aplicado. Dessa forma, obtém-se o método numérico híbrido que consiste em aplicar o método WENO para regiões que apresentam função descontínua e o método de diferenças centrais para regiões suaves, sendo que cada um destes apresentam qualidades importantes para a região em que devem ser aplicados. 
Para funções unidimensionais, o detector apresentou bom desempenho para os diversos casos testados, inclusive quando comparados com os métodos estudados durante a revisão bibliográfica, permitindo assim que fosse adaptado para o caso bidimenisonal. Assim como para as funções unidimensionais, o indicador apresentou bons resultados para os casos bidimensionais.

Com isso, com o detector de descontinuidades desenvolvido, as equações de Euler 1D e 2D para diferentes condições inicias foram resolvidas, permitindo avaliar através de diversos testes e gráficos os resultados obtidos através da solução numérica do método híbrido e da aplicação apenas do método WENO. Em diversos casos observou-se que o método numérico híbrido apresenta vantagem quanto comparado com a aplicação de apenas o método WENO.

Com o bom desempenho do método numérico híbrido e as principais vantagens que o mesmo apresenta, é possível observar melhores resultados com menos pontos na discretização, tanto para os casos unidimensionais como para os bidimensionais.

Através do desenvolvimento do novo detector de descontinuidades e da solução das equações de Euler unidimensionais e bidimensionais, é possível ainda que mais trabalhos possam ser realizados, tais como:

- Otimização do código que utiliza o método numérico híbrido desenvolvido;

- Aplicação em malhas generalizadas;

- Solução numérica das equações de Navier-Stokes. 
APÊNDICE

\section{$A$}

Método WENO

O método WENO [13] é uma modificação do método ENO [26], sendo que o último verifica, um a um, todos os pontos que são candidatos a fazer parte do estêncil para o cálculo da derivada espacial. Apesar do ENO apresentar um bom desempenho, essa verificação exige muitas sentenças lógicas, aumentando assim o custo computacional. Com isso, para evitar esse alto custo e manter o bom desempenho, o método WENO utiliza a combinação ponderada de estêncis menores para o cálculo da derivada.

Seja $\hat{f}(x)$ uma função interpoladora de $f(x)$ tal que

$$
f_{i}=\frac{1}{h} \int_{x_{i-1 / 2}}^{x_{i+1 / 2}} \hat{f}(s) d s .
$$

Calculando-se a derivada da equação (A.1) em relação a $x$ e aplicando-a no ponto $x_{i}$, tem-se

$$
f^{\prime}\left(x_{i}\right)=\frac{\hat{f}\left(x_{i+1 / 2}\right)-\hat{f}\left(x_{i-1 / 2}\right)}{h} .
$$

Com o objetivo de obter a combinação linear presente no método WENO, supõe-se $r$ estencêis, sendo cada um denotado como $S_{k}$, em que $k=0, \ldots, r-1$. Assim,

$$
S_{k}=\left\{x_{i+k-r+1}, x_{i+k-r+2}, \ldots, x_{i+k}\right\} .
$$

Dessa forma, tem-se para cada estêncil e os pontos associados um polinômio interpolador de $\hat{f}$

$$
p_{k}(x)=a_{k}\left(x-x_{c}\right)^{2}+b_{k}\left(x-x_{c}\right)+c_{k},
$$


tal que $x_{c}$ representa o ponto central. No caso em que o número de pontos é impar, escolhe-se o que se encontra ao centro do estêncil. Porém, quando o número de pontos é par, há duas possibilidades: $x_{n / 2}$ ou $x_{n / 2+1}$, devendo esta ser mantida para todos os estênceis.

Juntamente com a equação (A.1), é necessário que o polinômio (A.4) satisfaça

$$
\begin{aligned}
f\left(x_{i+k-r+1}\right) & =\frac{1}{h}\left(\int_{x_{i+k-r+1-1 / 2}}^{x_{i+k-r+1+1 / 2}} p_{k}(\xi) d \xi\right) \\
f\left(x_{i+k-r+2}\right) & =\frac{1}{h}\left(\int_{x_{i+k-r+2-1 / 2}}^{x_{i+k-r+2+1 / 2}} p_{k}(\xi) d \xi\right) . \\
\vdots & =\frac{1}{h}\left(\int_{x_{i+k-1 / 2}}^{x_{i+k+1 / 2}} p_{k}(\xi) d \xi\right)
\end{aligned}
$$

Considerando $r=3$, tem-se que $k=0,1,2$. Com isso, os estênceis para cada $k$ são

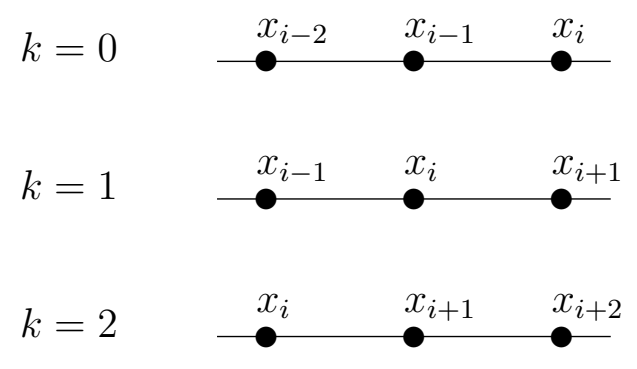

Para obter as constantes $a_{k}, b_{k}$ e $c_{k}$ presentes em (A.4) é necessário resolver os sistemas formados pela equação (A.5). Para resolver as integrais é necessário realizar uma troca de variável: $s=x-x_{c}$. Reescrevendo a eqaução (A.4), tem-se

$$
p_{k}(s)=a_{k} s^{2}+b_{k} s+c_{k}
$$

cuja solução dos sistemas para $k=0, k=1$ e $k=2$ resulta nas constantes

$$
\begin{aligned}
& \text { - } a_{0}=\frac{f_{i-2}-2 f_{i-1}+f_{i}}{2 h^{2}}, b_{0}=\frac{-f_{i-2}+f_{i}}{2 h} \text { e } c_{0}=\frac{-f_{i-2}+26 f_{i-1}-f_{i}}{24} ; \\
& \text { - } a_{1}=\frac{f_{i-1}-2 f_{i}+f_{i+1}}{2 h^{2}}, b_{1}=\frac{-f_{i-1}+f_{i+1}}{2 h} \text { e } c_{1}=\frac{-f_{i-1}+26 f_{i}-f_{i+1}}{24} ; \\
& \text { - } a_{2}=\frac{f_{i}-2 f_{i+1}+f_{i+2}}{2 h^{2}}, b_{2}=\frac{-f_{i}+f_{i+2}}{2 h} \text { e } c_{2}=\frac{-f_{i}+26 f_{i+1}-f_{i+2}}{24}
\end{aligned}
$$

Com o objetivo de calcular as aproximações de $\hat{f}_{i+1 / 2}$ para o estêncil $\left(x_{i-2}, x_{i-1}, x_{i}, x_{i+1}, x_{i+2}\right)$, é necessário conhecer $\hat{f}_{i+1 / 2}$ em cada estêncil menor, ou seja, quando $k=0, k=1 \mathrm{e} k=2$. Assim, 
- $\operatorname{para} k=0$

$$
\hat{f}_{i+1 / 2}=p_{0}\left(x_{i+1 / 2}\right),
$$

e assim obtém-se

$$
\hat{f}_{i+1 / 2}=\frac{1}{6}\left(2 f_{i-2}-7 f_{i-1}+11 f_{i}\right) .
$$

- $\operatorname{para} k=1$

$$
\hat{f}_{i+1 / 2}=p_{1}\left(x_{i+1 / 2}\right),
$$

e assim obtém-se

$$
\hat{f}_{i+1 / 2}=\frac{1}{6}\left(-f_{i-1}+5 f_{i}+2 f_{i+1}\right) .
$$

- $\operatorname{para} k=2$

$$
\hat{f}_{i+1 / 2}=p_{2}\left(x_{i+1 / 2}\right),
$$

e assim obtém-se

$$
\hat{f}_{i+1 / 2}=\frac{1}{6}\left(2 f_{i}+5 f_{i+1}-f_{i+2}\right) .
$$

Dessa forma, é possível escrever $\hat{f}_{i+1 / 2}$ para o estêncil $\left(x_{i-2}, x_{i-1}, x_{i}, x_{i+1}, x_{i+2}\right)$ como combinação linear das aproximações do mesmo para os estênceis menores, tal que

$$
\begin{aligned}
\hat{f}_{i+1 / 2}=\omega_{0}\left[\frac{1}{6}\left(2 f_{i-2}-7 f_{i-1}+11 f_{i}\right)\right] & +\omega_{1}\left[\frac{1}{6}\left(-f_{i-1}+5 f_{i}+2 f_{i+1}\right)\right]+ \\
& +\omega_{2}\left[\frac{1}{6}\left(2 f_{i}+5 f_{i+1}-f_{i+2}\right)\right]
\end{aligned}
$$

O cálculo de $\hat{f}_{i-1 / 2}$ é feito de maneira similar, apenas deslocando os termos da equação (A.13), resultando em

$$
\begin{aligned}
\hat{f}_{i-1 / 2}=\omega_{0}\left[\frac{1}{6}\left(2 f_{i-3}-7 f_{i-2}+11 f_{i-1}\right)\right]+\omega_{1} & {\left[\frac{1}{6}\left(-f_{i-2}+5 f_{i-1}+2 f_{i}\right)\right]+} \\
+ & +\omega_{2}\left[\frac{1}{6}\left(2 f_{i-1}+5 f_{i}-f_{i+1}\right)\right] .
\end{aligned}
$$

Para calcular os pesos $\omega_{k}$, é necessário determinar os pesos ideais $C_{k}$ que são obtidos através da comparação da equação (A.13) com o polinômio $p_{2}\left(x_{i+1 / 2}-x_{i}\right)$ para o caso em que $r=5$, ou seja, $k=0,1,2,3,4$. Dessa forma, os pesos ideais são: $C_{0}=1 / 10, C_{1}=6 / 10$ e $C_{2}=3 / 10$.

A fim de avaliar a ordem do método WENO considerado, tem-se $f_{i}^{\prime}$ determinado com os pesos ideais:

$$
f_{i}^{\prime}=\frac{1}{60 h}\left[-2 f_{i-3}+15 f_{i-2}-60 f_{i-1}+20 f_{i}+30 f_{i+1}-3 f_{i+2}\right]
$$


com erro de truncamento

$$
\tau_{W E N O}=\frac{1}{60} d x^{5} f^{(6)}-\frac{1}{140} d x^{6} f^{(7)}+\frac{1}{240} d x^{7} f^{(8)}+O\left(d x^{8}\right)
$$

Observa-se que essa aproximação é de ordem 5 com termos dissipativos e dispersivos, sendo que o método WENO é considerado como dissipativo pelo fato do termo de menor ordem possuir derivada par. Com os pesos ideais calculados, é possível obter $\omega_{k}$,

$$
\omega_{k}=\frac{\alpha_{k}}{\alpha_{0}+\cdots+\alpha_{r-1}} \operatorname{com} \alpha_{k}=\frac{C_{k}}{\left(\varepsilon+I S_{k}\right)^{p}}
$$

$\operatorname{com} p=2$ e $\varepsilon=10^{-6}$. Os valores de $I S_{k}$ são obtidos por

$$
I S_{k}=\sum_{l=1}^{l=r-1} \int_{x_{i-1 / 2}}^{x_{i+1 / 2}} d x^{2 l-1}\left(p_{k}^{(l)}(\xi)\right)^{2} d \xi
$$

em que $p_{k}^{(l)}(\xi)$ é a $l-$ sima derivada do polinômio.

Para $r=3$, os valores de $I S_{k}$ são

$$
\begin{aligned}
& I S_{0}=\frac{13}{12}\left(f_{i-2}-2 f_{i-1}+f_{i}\right)^{2}+\frac{1}{4}\left(f_{i-2}-4 f_{i-1}+3 f_{i}\right)^{2}, \\
& I S_{1}=\frac{13}{12}\left(f_{i-1}-2 f_{i}+f_{i+1}\right)^{2}+\frac{1}{4}\left(f_{i-1}-f_{i+1}\right)^{2} \\
& I S_{2}=\frac{13}{12}\left(f_{i}-2 f_{i+1}+f_{i+2}\right)^{2}+\frac{1}{4}\left(3 f_{i}-4 f_{i+1}+f_{i+2}\right)^{2} .
\end{aligned}
$$

\section{A.1 Análise de Fourier}

Para avaliar e obter mais informações sobre o método, é realizada uma análise de Fourier. Considerando-se a função $f(x)$ periódica, ou seja, $f_{1}=f_{N_{x}+1}$ e o domínio $[0, L]$ tal que $x_{1}=0$, $x_{N_{x}}=L$ e $h=L / N_{x}$, a função $f(x)$ em série de Fourier é

$$
f(x)=\sum_{k=-N_{x} / 2}^{k=N_{x} / 2} f_{k} \exp \left(\frac{2 \pi i k x}{L}\right), i=\sqrt{-1} .
$$

Sem perda de generalidade, é possível considerar apenas um elemento $k$ do somatório apresentado na equação (2.10), sendo este

$$
F(x)=\exp \left(\frac{2 \pi i k x}{L}\right)
$$


Seja $x_{j}=(j-1) h$ e o número de onda dado por

$$
w=\frac{2 \pi k h}{L}=\frac{2 \pi k}{N_{x}}
$$

A expressão (2.11) calculado no ponto $x_{j}$ é tal que

$$
F_{j}=\exp \left(\frac{2 \pi i k j}{N_{x}}\right)=\exp (i w j)
$$

Da mesma forma calcula-se $F(x)$ para o ponto $x_{j+l}$, cuja expressão é

$$
F_{j+l}=\exp \left(\frac{2 \pi i k(j+l)}{N_{x}}\right)=\exp \left(\frac{2 \pi i k l}{N_{x}}\right) F_{j}=\exp (i w l) F_{j} .
$$

Assim, é possível determinar as derivadas em relação a $x$ das equações (A.23) e (A.24) através da regra da cadeia,

$$
F_{j}^{\prime}=\frac{i w^{*}}{h} F_{j}
$$

e

$$
F_{j+l}^{\prime}=\frac{i w^{*}}{h} F_{j} \exp (i w l) .
$$

Para o método WENO substitui-se as equações (A.23), (A.24), (A.25) e (A.26) na expressão de $f_{i}^{\prime}$ da equação (A.15), sendo

- Parte real

$$
\Re\left(w^{*}(w)\right)=\frac{1}{30}(45 \operatorname{sen}(w)-9 \operatorname{sen}(2 w)+\operatorname{sen}(3 w)) ;
$$

- Parte imaginária

$$
\Im\left(w^{*}(w)\right)=\frac{1}{30}(-10+15 \cos (w)-6 \cos (2 w)+\cos (3 w)) .
$$

A Figura A.1 mostra os gráficos obtidos através das equações (A.27) e (A.28).

Nota-se que na Figura A.1(a) a reta representa a função descontínua em que $w^{*}(\pi)=0$ e $w^{*}(w)=w$, para $w \neq \pi$; já a curva representa a função para o método WENO de ordem 5. A Figura A.1(b) representa a parte imaginária da análise de Fourier, sendo que a reta é dada por $w^{*}(w)=0$; já a curva representa a parte imaginária da função para o método WENO de ordem 5. 


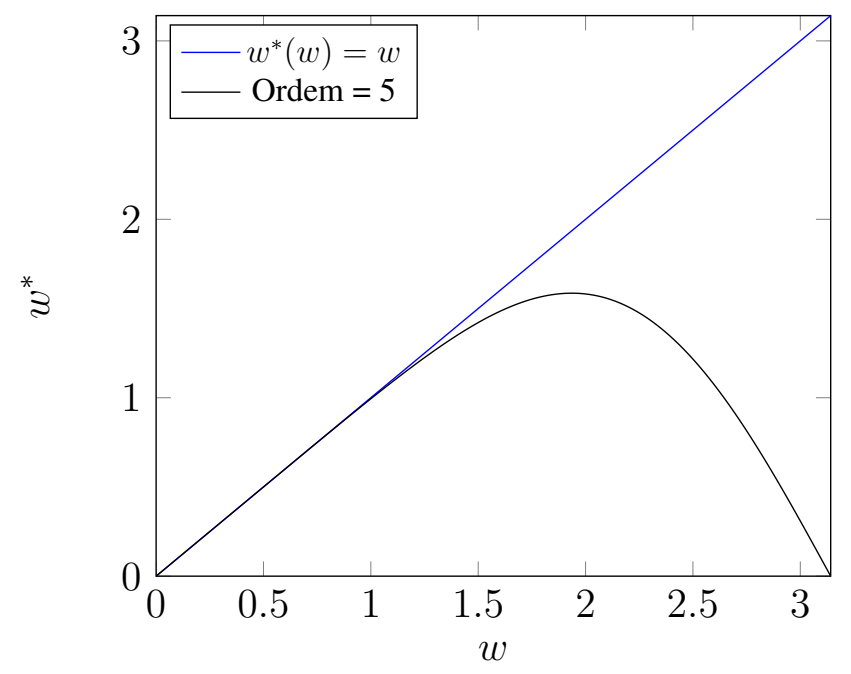

(a) WENO - Parte Real

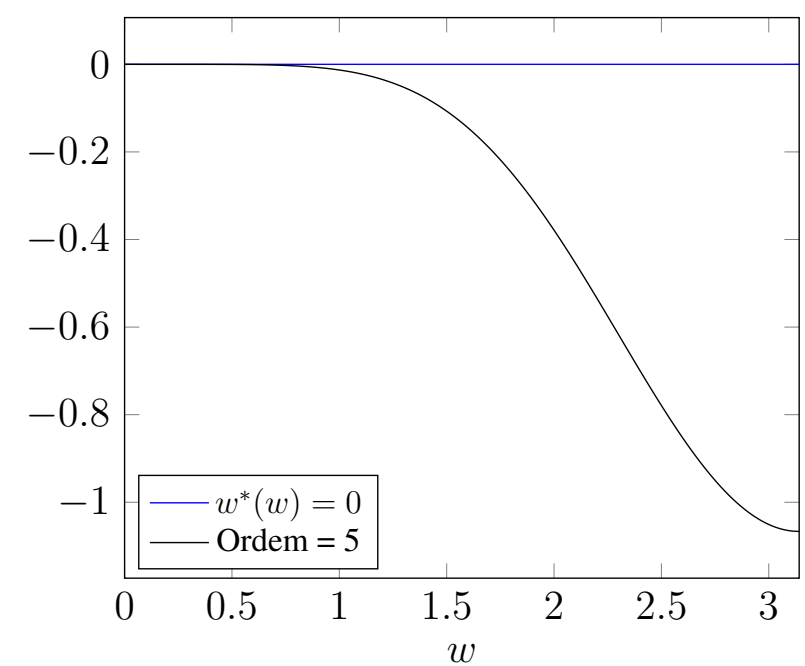

(b) WENO - Parte Imaginária

Figura A.1: Análise de Fourier - WENO 
APÊNDICE

\section{$B$}

Equações de Euler

A seguir são descritas as equações de Euler para os casos unidimensional e bidimensional.

\section{B.1 Caso Unidimensional}

Com a finalidade de descrever as equações de Euler para o caso unidimensional, as mesmas são apresentadas na forma vetorial com a descrição da sepração de fluxo desenvolvida por StegerWarming em [28]. Além disso, é apresentado um algoritmo do método híbrido desenvolvido para o cado $1 \mathrm{D}$.

\section{B.1.1 Forma Vetorial}

As equações de Euler 1D na forma vetorial são dadas por

$$
\frac{\partial \mathbf{U}}{\partial t}+\frac{\partial \mathbf{F}}{\partial x}=\mathbf{0}
$$

onde

$$
\mathbf{U}=\left[\begin{array}{c}
\rho \\
\rho u_{x} \\
\rho e_{T}
\end{array}\right], \quad \mathbf{F}=\left[\begin{array}{c}
\rho u_{x} \\
p+\rho u_{x}^{2} \\
u_{x}\left(\rho e_{T}+p\right)
\end{array}\right]
$$

Nota-se pelos vetores apresentados na equação (B.2) que há três equações e quatro incógnitas: densidade $(\rho)$, velocidade $\left(u_{x}\right)$, pressão $(p)$ e energia $\left(e_{T}\right)$. Assim, é necessário construir uma relação entre duas incógnitas que permite resolver as equações de Euler. 


\section{B.1.2 Equação de Estado}

Através da equação de estado, é possível relacionar energia e pressão da seguinte maneira

$$
p=\rho R T
$$

em que $R$ é uma constante e $T$ a temperatura. Além disso, é necessária mais uma equação

$$
e_{I}=c_{v} T
$$

tal que $c_{v}$ é o calor específico a um certo volume constante e que depende diretamente do fluido considerado.

Com isso, a relação entre $R$ e $c_{v}$ é definida da seguinte forma

$$
c_{p}=R+c_{v} \text { e } \gamma=\frac{c_{p}}{c_{v}}, \text { e assim } c_{v}=\frac{R}{\gamma-1},
$$

sendo que $c_{p}$ é o calor específico a uma certa pressão constante.

Ao isolar a temperatura ( $T$ ) na equação (B.4) e substituir em (B.3), obtém-se

$$
p=\rho R \frac{e_{I}}{c_{v}},
$$

e substituindo a equação (B.5) em (B.6), tem-se a relação entre a energia interna $\left(e_{I}\right)$ e a pressão ( $p$ ) dada por

$$
p=\rho e_{I}(\gamma-1)
$$

com $\gamma$ constante.

Assim,

$$
p=(\gamma-1)\left(\rho e_{T}-\frac{1}{2} \rho u_{x}^{2}\right),
$$

é a equação que relaciona energia e pressão, permitindo que o sistema de equações possa ser resolvido. Portanto, o vetor $\mathbf{F}$ pode ser reescrito tal que

$$
\mathbf{F}=\left[\begin{array}{c}
\rho u_{x} \\
(\gamma-1)\left(\rho e_{T}-\frac{1}{2} \rho u_{x}^{2}\right)+\rho u_{x}^{2} \\
u_{x}\left[\rho e_{T}+(\gamma-1)\left(\rho e_{T}-\frac{1}{2} \rho u_{x}^{2}\right)\right]
\end{array}\right] .
$$


Para um gás ideal, existe a equação de estado para a velocidade do som $(c)$, tal que

$$
c^{2}=\gamma R T \Rightarrow c^{2}=\frac{\gamma p}{\rho} \Rightarrow c=\sqrt{\frac{\gamma p}{\rho}}
$$

envolvendo densidade $(\rho)$ e pressão $(p)$.

\section{B.1.3 Separação de Fluxo}

Para realizar a implementação, foi estudado o método da separação de fluxo (F) desenvolvido por Steger Warming em [28]. Assim, a equação (B.1) pode ser reescrita tal que

$$
\frac{\partial \mathbf{U}}{\partial t}+A \frac{\partial \mathbf{U}}{\partial x}=\mathbf{0}
$$

sendo $A$ a matriz Jacobiana dada por $A=\frac{\partial \mathbf{F}}{\partial \mathbf{U}}$.

Dessa forma, calculando-se a matriz Jacobiana $A$, obtém-se

$$
A=\left[\begin{array}{ccc}
0 & 1 & 0 \\
u_{x}^{2} \frac{(\gamma-3)}{2} & u_{x}(3-\gamma) & \gamma-1 \\
-\gamma u_{x} e_{T}+(\gamma-1) u_{x}^{3} & \gamma e_{T}-\frac{3}{2}(\gamma-1) u_{x}^{2} & \gamma u_{x}
\end{array}\right]
$$

Com a equação (B.11) e a matriz Jacobiana calculada, a principal ideia do método da separação de fluxo é dividir o vetor $\mathbf{F}$ em duas parcelas tal que

$$
\mathbf{F}=\mathbf{F}^{+}+\mathbf{F}^{-}
$$

com $\mathbf{F}^{+}$e $\mathbf{F}^{-}$representando o fluxo positivo e negativo, respectivamente.

Através dessa ideia, é possível afirmar que a matriz $A$ também pode ser decomposta em duas partes, sendo $A=A^{+}+A^{-}$. As matrizes $A^{+}$e $A^{-}$possuem autovalores positivos e negativos, respectivamente.

Assim, a equação (B.11) pode ser reescrita tal que

$$
\frac{\partial \mathbf{U}}{\partial t}+A^{+} \frac{\partial \mathbf{U}}{\partial x}+A^{-} \frac{\partial \mathbf{U}}{\partial x}=\mathbf{0}
$$


Com as equações (B.8) e (B.10), a matriz Jacobiana $A$ é dada por

$$
A=\left[\begin{array}{ccc}
0 & 1 & 0 \\
u_{x}^{2} \frac{(\gamma-3)}{2} & u_{x}(3-\gamma) & \gamma-1 \\
\frac{u_{x}^{2}(\gamma-2)}{2}-\frac{c^{2} u_{x}}{\gamma-1} & u_{x}^{2}(3-2 \gamma)+\frac{c^{2}}{\gamma-1} & \gamma u_{x}
\end{array}\right]
$$

cujos autovalores são

$$
\begin{gathered}
\lambda_{1}=u_{x} \\
\lambda_{2}=u_{x}+c . \\
\lambda_{3}=u_{x}-c
\end{gathered}
$$

Através dos autovalores e da matriz $A$, pode-se calcular os autovetores associados, tal que

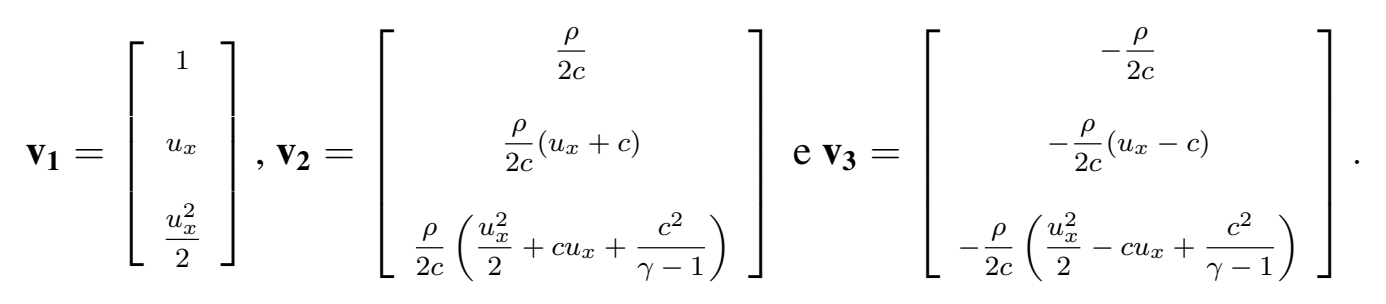

Observa-se que a matriz Jacobiana $A$ é diagonalizável, o que permite definir uma matriz $\Lambda$ diagonal dos autovalores de $A$ e uma matriz $T$ cujas colunas são os autovetores dessa matriz associados, tal que

$$
\Lambda=\left[\begin{array}{ccc}
u_{x} & 0 & 0 \\
0 & u_{x}+c & 0 \\
0 & 0 & u_{x}-c
\end{array}\right] \text { e } T=\left[\begin{array}{ccc}
1 & \frac{\rho}{2 c} & \frac{\rho}{2 c} \\
u_{x} & \left(u_{x}+c\right) \frac{\rho}{2 c} & -\left(u_{x}-c\right) \frac{\rho}{2 c} \\
\frac{u_{x}^{2}}{2} & \left(\frac{u_{x}^{2}}{2}+c u_{x}+\frac{c^{2}}{\gamma-1}\right) \frac{\rho}{2 c} & -\left(\frac{u_{x}^{2}}{2}-c u_{x}+\frac{c^{2}}{\gamma-1}\right) \frac{\rho}{2 c}
\end{array}\right] .
$$

Dessa forma, com a diagonalização da matriz $A$ e com a separação de fluxo, obtém-se

$$
A=T \Lambda^{+} T^{-1}+T \Lambda^{-} T^{-1}
$$

em que $\Lambda^{+}$é uma matriz com os autovalores positivos e $\Lambda^{-}$com os autovalores negativos. As expressões para essas matrizes são dadas por

$$
\Lambda^{+}=\left[\begin{array}{ccc}
\lambda_{1}^{+} & 0 & 0 \\
0 & \lambda_{2}^{+} & 0 \\
0 & 0 & \lambda_{3}^{+}
\end{array}\right] \text {e } \Lambda^{-}=\left[\begin{array}{ccc}
\lambda_{1}^{-} & 0 & 0 \\
0 & \lambda_{2}^{-} & 0 \\
0 & 0 & \lambda_{3}^{-}
\end{array}\right]
$$


Assim, $\lambda_{i}^{+}=\frac{1}{2}\left(\lambda_{i}+\left|\lambda_{i}\right|\right)$ são os autovalores positivos e $\lambda_{i}^{-}=\frac{1}{2}\left(\lambda_{i}-\left|\lambda_{i}\right|\right)$ são os autovalores negativos da matriz $A$, tal que

$$
\mathbf{F}^{ \pm}=\frac{(\gamma-1)}{\gamma} \rho \lambda_{1}^{ \pm}\left[\begin{array}{c}
1 \\
u_{x} \\
\frac{u_{x}^{2}}{2}
\end{array}\right]+\frac{\rho}{2 \gamma} \lambda_{2}^{ \pm}\left[\begin{array}{c}
1 \\
u_{x}+c \\
\frac{u_{x}^{2}}{2}+c u_{x}+\frac{c^{2}}{\gamma-1}
\end{array}\right]+\frac{\rho}{2 \gamma} \lambda_{3}^{ \pm}\left[\begin{array}{c}
1 \\
u_{x}-c \\
\frac{u_{x}^{2}}{2}-c u_{x}+\frac{c^{2}}{\gamma-1}
\end{array}\right] .
$$

Com a separação de fluxo e as equações de Euler para o caso unidimensinal definidas, é possível então resolvê-las numericamente através do método híbrido com o novo detector desenvolvido.

\section{B.1.4 Algoritmo}

Para resolver numericamente as equações de Euler 1D apresentadas em (B.1), tem-se o seguinte algoritmo.

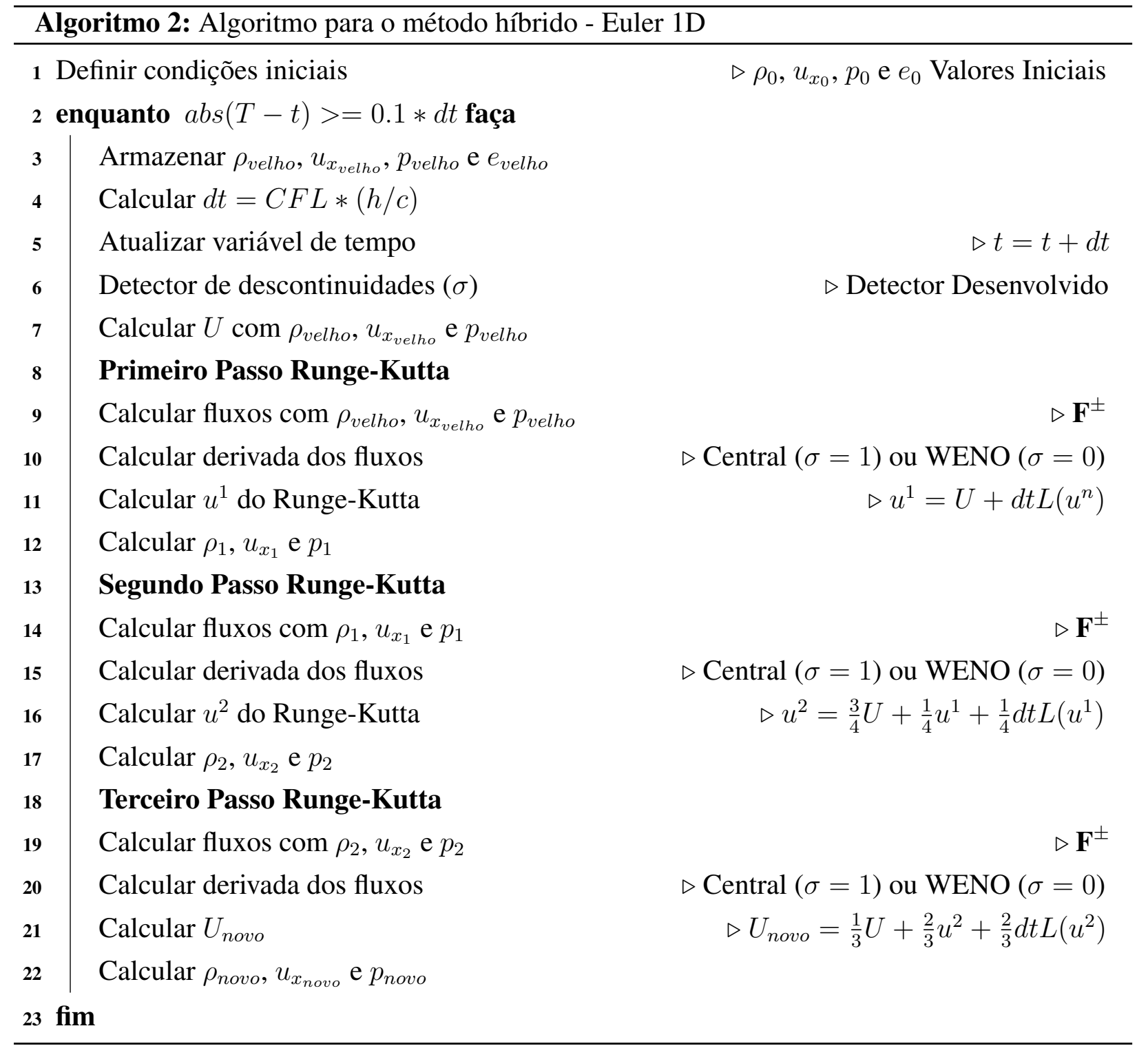

Observa-se que para o cálculo das derivadas espacias são utilizados os métodos WENO ou diferenças centrais com a aplicação do novo detector de descontinuidades desenvolvido. Já para o 
cálculo da derivada temporal, o método de Runge-Kutta TVD de ordem 3 [13] é implementado. As variáveis $T$ e $d t$ indicam o tempo final e a variação do tempo, respectivamente.

\section{B.2 Caso Bidimensional}

Com a finalidade de descrever as equações de Euler para o caso bidimensional, as mesmas são apresentadas na forma vetorial com a descrição da sepração de fluxo desenvolvida por StegerWarming em [28]. Além disso, é apresentado um algoritmo do método híbrido desenvolvido para o cado $2 \mathrm{D}$.

\section{B.2.1 Forma Vetorial}

As equações de Euler 2D na forma vetorial são dadas por

$$
\frac{\partial \mathbf{U}}{\partial t}+\frac{\partial \mathbf{F}}{\partial x}+\frac{\partial \mathbf{G}}{\partial y}=\mathbf{0}
$$

tal que

$$
\mathbf{U}=\left[\begin{array}{c}
\rho \\
\rho u_{x} \\
\rho u_{y} \\
\rho e_{T}
\end{array}\right], \quad \mathbf{F}=\left[\begin{array}{c}
\rho u_{x} \\
p+\rho u_{x}^{2} \\
\rho u_{x} u_{y} \\
u_{x}\left(\rho e_{T}+p\right)
\end{array}\right], \quad \mathbf{G}=\left[\begin{array}{c}
\rho u_{y} \\
\rho u_{x} u_{y} \\
p+\rho u_{y}^{2} \\
u_{y}\left(\rho e_{T}+p\right)
\end{array}\right]
$$

Nota-se pelos vetores apresentados na equação (B.23) que há quatro equações e cinco incógnitas: densidade $(\rho)$, velocidade na direção $x\left(u_{x}\right)$, velocidade na direção $y\left(u_{y}\right)$, pressão $(p)$ e energia $\left(e_{T}\right)$. Assim, é necessário construir uma relação entre duas incógnitas que permite resolver as equações de Euler.

\section{B.2.2 Equação de Estado}

Através da equação de estado, é possível relacionar energia e pressão da seguinte maneira

$$
p=\rho R T
$$

em que $R$ é uma constante e $T$ a temperatura. Além disso, é necessária mais uma equação

$$
e_{I}=c_{v} T
$$

tal que $c_{v}$ é o calor específico a um certo volume constante e que depende diretamente do fluido considerado. 
Com isso, a relação entre $R$ e $c_{v}$ é definida da seguinte forma

$$
c_{p}=R+c_{v} \text { e } \gamma=\frac{c_{p}}{c_{v}}, \text { e assim } \quad c_{v}=\frac{R}{\gamma-1},
$$

sendo que $c_{p}$ é o calor específico a uma certa pressão constante.

Ao isolar a temperatura (T) na equação (B.25) e substituir em (B.24), obtém-se

$$
p=\rho R \frac{e_{I}}{c_{v}}
$$

e substituindo a equação (B.26) em (B.27), tem-se a relação entre a energia interna $\left(e_{I}\right)$ e a pressão ( $p$ ) dada por

$$
p=\rho e_{I}(\gamma-1),
$$

com $\gamma$ constante.

Assim,

$$
p=(\gamma-1)\left[\rho e_{T}-\frac{\rho}{2}\left(u_{x}^{2}+u_{y}^{2}\right)\right],
$$

é a equação que relaciona energia e pressão, permitindo que o sistema de equações possa ser resolvido. Portanto, os vetores $\mathbf{F}$ e $\mathbf{G}$ podem ser reescritos tal que

$$
\mathbf{F}=\left[\begin{array}{c}
\rho u_{x} \\
(\gamma-1)\left(\rho e_{T}-\frac{\rho}{2}\left(u_{x}^{2}+u_{y}^{2}\right)\right)+\rho u_{x}^{2} \\
\rho u_{x} u_{y} \\
u_{x}\left[\rho e_{T}+(\gamma-1)\left(\rho e_{T}-\frac{\rho}{2}\left(u_{x}^{2}+u_{y}^{2}\right)\right)\right]
\end{array}\right], \mathbf{G}=\left[\begin{array}{c}
\rho u_{x} \\
\rho u_{x} u_{y} \\
(\gamma-1)\left(\rho e_{T}-\frac{\rho}{2}\left(u_{x}^{2}+u_{y}^{2}\right)\right)+\rho u_{y}^{2} \\
u_{y}\left[\rho e_{T}+(\gamma-1)\left(\rho e_{T}-\frac{\rho}{2}\left(u_{x}^{2}+u_{y}^{2}\right)\right)\right]
\end{array}\right] .
$$

Para um gás ideal, existe a equação de estado para a velocidade do som $(c)$, tal que

$$
c^{2}=\gamma R T \Rightarrow c^{2}=\frac{\gamma p}{\rho} \Rightarrow c=\sqrt{\frac{\gamma p}{\rho}}
$$

envolvendo densidade $(\rho)$ e pressão $(p)$.

\section{B.2.3 Separação de Fluxo}

Para realizar a implementação das equações de Euler bidimensionais, foi estudado o método da separação de fluxo desenvolvido por Steger-Warming [28]. Com isso, a equação (B.22) pode ser reescrita tal

$$
\frac{\partial \mathbf{U}}{\partial t}+A \frac{\partial \mathbf{U}}{\partial x}+B \frac{\partial \mathbf{U}}{\partial y}=\mathbf{0}
$$

sendo $A$ e $B$ matrizes Jacobianas dadas por $A=\frac{\partial \mathbf{F}}{\partial \mathbf{U}}$ e $B=\frac{\partial \mathbf{G}}{\partial \mathbf{U}}$, respectivamente. 
Com a equação (B.32) e as matrizes $A$ e $B$ determinadas, a ideia do método da separação de fluxo é dividir os vetores $\mathbf{F}$ e $\mathbf{G}$ em duas parcelas, tal que

$$
\mathbf{F}=\mathbf{F}^{+}+\mathbf{F}^{-} \text {e } \mathbf{G}=\mathbf{G}^{+}+\mathbf{G}^{-}
$$

com $\mathbf{F}^{+}$e $\mathbf{G}^{+}$representando o fluxo positivo e $\mathbf{F}^{-}$e $\mathbf{G}^{-}$o fluxo negativo.

Assim, é possível afirmar que as matrizes $A$ e $B$ também podem ser decompostas em duas partes, sendo $A=A^{+}+A^{-}$e $B=B^{+}+B^{-}$. As matrizes $A^{+}$e $B^{+}$possuem autovalores positivos e $A^{-}$e $B^{-}$autovalores negativos.

Portanto, a equação (B.32) pode ser reescrita da seguinte maneira

$$
\frac{\partial \mathbf{U}}{\partial t}+A^{+} \frac{\partial \mathbf{U}}{\partial x}+A^{-} \frac{\partial \mathbf{U}}{\partial x}+B^{+} \frac{\partial \mathbf{U}}{\partial y}+B^{-} \frac{\partial \mathbf{U}}{\partial y}=\mathbf{0} .
$$

Da mesma forma que no caso unidimensional, é possível obter os autovalores das matrizes Jacobianas $A$ e $B$, sendo que

$$
\begin{gathered}
\lambda_{1_{A}}=\lambda_{2_{A}}=u_{x} \\
\lambda_{3_{A}}=u_{x}+c \\
\lambda_{4_{A}}=u_{x}-c
\end{gathered}
$$

são os autovalores da matriz A, e

$$
\begin{gathered}
\lambda_{1_{B}}=\lambda_{2_{B}}=u_{y} \\
\lambda_{3_{B}}=u_{y}+c \\
\lambda_{4_{B}}=u_{y}-c
\end{gathered}
$$

são os autovalores da matriz $B$.

Observa-se que as matrizes $A$ e $B$ são diagonalizáveis, o que permite definir $\Lambda_{A}$ e $\Lambda_{B}$ como sendo matrizes diagonais dos autovalores e $T_{A}$ e $T_{B}$ dos autovetores associados a $A$ e $B$, respectivamente. Dessa forma, juntamente com a sepração do fluxo obtém-se

$$
A=T_{A} \Lambda_{A}^{+} T_{A}^{-1}+T_{A} \Lambda_{A}^{-} T_{A}^{-1} \text { e } B=T_{B} \Lambda_{B}^{+} T_{B}^{-1}+T_{B} \Lambda_{B}^{-} T_{B}^{-1}
$$

em que $\Lambda_{A}^{+}$e $\Lambda_{B}^{+}$são os autovalores positivos e $\Lambda_{A}^{-}$e $\Lambda_{B}^{-}$os autovalores negativos das matrizes $A$ e $B$, respectivamente. As expressões para essas matrizes são dadas por

$$
\Lambda_{A, B}^{+}=\left[\begin{array}{cccc}
\lambda_{1_{A, B}}^{+} & 0 & 0 & 0 \\
0 & \lambda_{2_{A, B}}^{+} & 0 & 0 \\
0 & 0 & \lambda_{3_{A, B}}^{+} & 0 \\
0 & 0 & 0 & \lambda_{4_{A, B}}^{+}
\end{array}\right] \mathrm{e} \Lambda_{A, B}^{-}=\left[\begin{array}{cccc}
\lambda_{1_{A, B}}^{-} & 0 & 0 & 0 \\
0 & \lambda_{2_{A, B}}^{-} & 0 & 0 \\
0 & 0 & \lambda_{3_{A, B}}^{-} & 0 \\
0 & 0 & 0 & \lambda_{4_{A, B}}^{-}
\end{array}\right]
$$

Assim, $\lambda_{i_{A, B}}^{+}=\frac{1}{2}\left(\lambda_{i_{A, B}}+\left|\lambda_{i_{A, B}}\right|\right)$ são os autovalores positivos e $\lambda_{i_{A, B}}^{-}=\frac{1}{2}\left(\lambda_{i_{A, B}}-\left|\lambda_{i_{A, B}}\right|\right)$ são os autovalores negativos de $\mathrm{A}$ e $\mathrm{B}$, tal que 
$\mathbf{F}^{ \pm}=\frac{(\gamma-1)}{\gamma} \rho \lambda_{1_{A}}^{ \pm}\left[\begin{array}{c}1 \\ u_{x} \\ u_{y} \\ \frac{u_{x}^{2}+u_{y}^{2}}{2}\end{array}\right]+\frac{\rho}{2 \gamma} \lambda_{3_{A}}^{ \pm}\left[\begin{array}{c}1 \\ u_{x}+c \\ u_{y} \\ \frac{\left(u_{x}+c\right)^{2}}{2}+\frac{u_{y}^{2}}{2}+\frac{(3-\gamma) c^{2}}{2(\gamma-1)}\end{array}\right]+\frac{\rho}{2 \gamma} \lambda_{4_{A}}^{ \pm}\left[\begin{array}{c}1 \\ u_{x}-c \\ u_{y} \\ \frac{\left(u_{x}-c\right)^{2}}{2}+\frac{u_{y}^{2}}{2}+\frac{(3-\gamma) c^{2}}{2(\gamma-1)}\end{array}\right]$

$\mathrm{e}$

$\mathbf{G}^{ \pm}=\frac{(\gamma-1)}{\gamma} \rho \lambda_{1_{B}}^{ \pm}\left[\begin{array}{c}1 \\ u_{x} \\ u_{y} \\ \frac{u_{x}^{2}+u_{y}^{2}}{2}\end{array}\right]+\frac{\rho}{2 \gamma} \lambda_{3_{B}}^{ \pm}\left[\begin{array}{c}1 \\ u_{x} \\ u_{y}+c \\ \frac{u_{x}^{2}}{2}+\frac{\left(u_{y}+c\right)^{2}}{2}+\frac{(3-\gamma) c^{2}}{2(\gamma-1)}\end{array}\right]+\frac{\rho}{2 \gamma} \lambda_{4_{B}}^{ \pm}\left[\begin{array}{c}1 \\ u_{x} \\ u_{y}-c \\ \frac{\left(u_{y}-c\right)^{2}}{2}+\frac{(3-\gamma) c^{2}}{2(\gamma-1)}\end{array}\right]$.

Com a separação de fluxo e as equações de Euler para o caso bidimensional definidas, é possível então resolvê-las numericamente através do método híbrido com o novo detector desenvolvido. 


\section{B.2.4 Algoritmo}

Para resolver numericamente as equações de Euler 2D apresentadas em (B.22), tem-se o seguinte algoritmo.

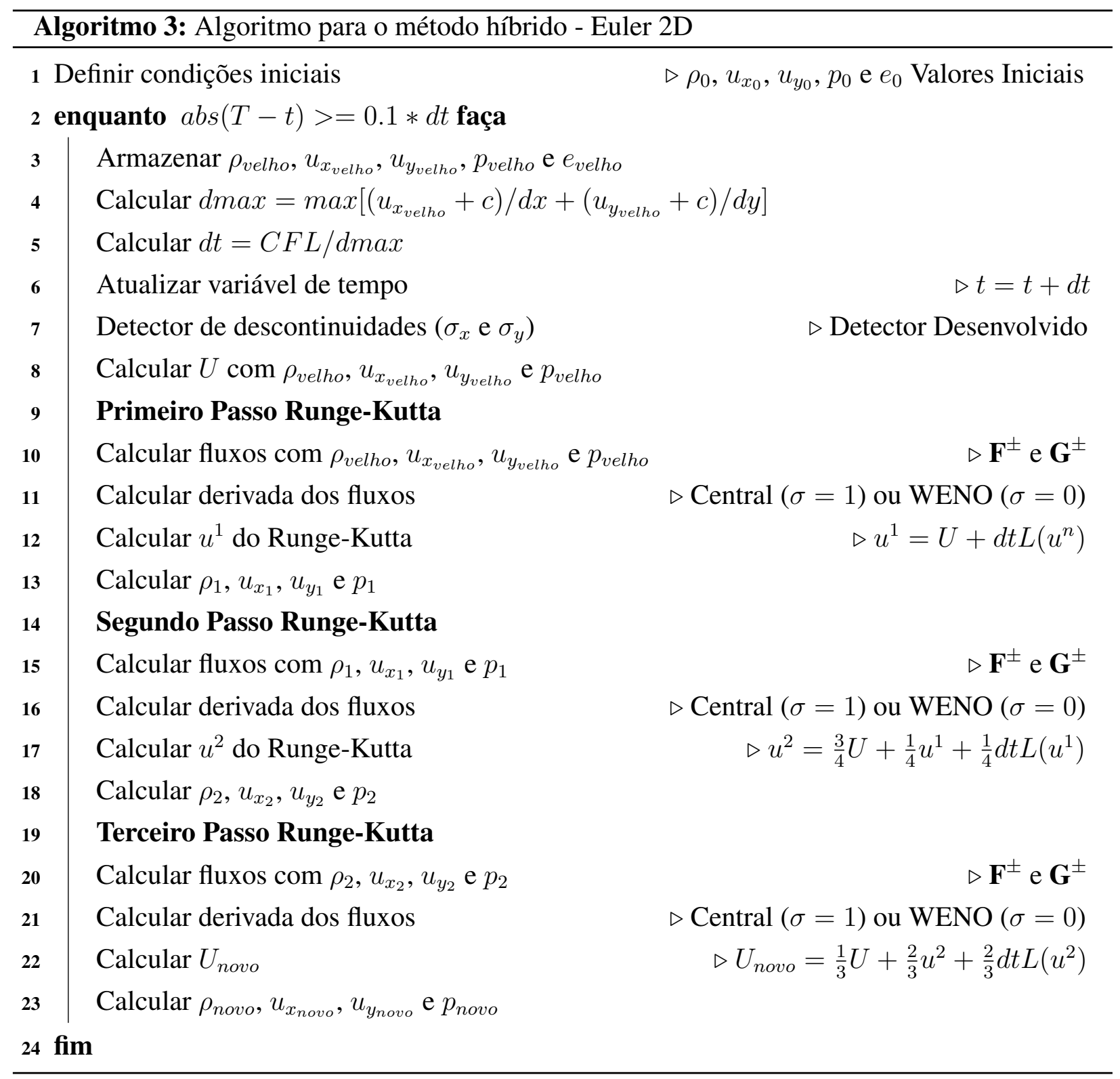

Assim como o caso 1D, para o cálculo das derivadas espaciais são utilizados os métodos WENO e diferenças centrais com a aplicação do detector de descontinuidades desenvolvido. $\mathrm{O}$ cálculo da derivada temporal é realizado com o método de Runge-Kutta TVD de ordem 3 [13]. As variáveis $T$ e $d t$ representam o tempo final e a variação do tempo, respectivamente. 
APÊNDICE

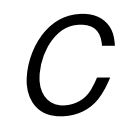

\section{Detectores de Descontinuidades}

A seguir são descritos os detectores de descontinuidades de Adams [2], Yee [33], Kim [14], Hill [11], Pirozzoli [21] e Zhou [36].

\section{C.1 Adams}

Em [2] define-se um indicador de suavidade da seguinte maneira:

$$
r_{i}=\frac{\Delta f_{i+1}}{h}
$$

em que $\Delta f_{i+1}=f_{i+1}-f_{i}$ e $h=x_{i+1}-x_{i}$.

Com isso, se as desigualdades

$$
\begin{gathered}
\left|r_{i}\right| h>\varepsilon \\
\left|r_{i}\right|>\left|r_{i-1}\right| \text { e }\left|r_{i}\right| \geq\left|r_{i+1}\right|
\end{gathered}
$$

forem sastisfeitas, é necessário aplicar um método para regiões com função descontínua, ou seja, $\sigma_{i}=0$, já que o produto do módulo do gradiente com o espaçamento da malha é maior do que o valor limiar $\varepsilon$ e o gradiente atinge um máximo local.

Caso uma dessas desigualdades não for satisfeita, aplica-se então um método para regiões com função suave, ou seja, $\sigma_{i}=1$.

Para realizar a combinação linear do método numérico híbrido, em [2] foi escolhido o método Compacto [16] e ENO [26] para serem aplicados nas regiões com função suave e descontínua, respectivamente. Dessa maneira, o detector tem a importante função de determinar quais regiões 
podem ser consideradas com função suave e quais com função descontínua, para que assim um dos métodos possa ser escolhido e consequentemente a derivada espacial calculada.

\section{C.2 Yee}

No caso do detector apresentado em [33], este não é utilizado em métodos numéricos híbridos, mas sim na aplicação de um filtro. Assim, para resolver as derivadas espaciais, aplica-se um método que apresenta oscilações e depois com a função detectora determina-se as regiões descontínuas para a aplicação do filtro. O principal objetivo é diminuir as oscilações resultantes.

Para definir se uma determinada região pode ser considerada com função suave ou descontínua, define-se o seguinte indicador de suavidade:

$$
\hat{r}_{i}=\left(\frac{|| \Delta f_{i+1}|-| \Delta f_{i}||}{\left(\left|\Delta f_{i+1}\right|+\left|\Delta f_{i}\right|\right)+\varepsilon}\right)^{p}
$$

em que $\Delta f_{i+1}=f_{i+1}-f_{i}$ e $\varepsilon$ um número real positivo para evitar uma possível divisão por zero.

Observa-se, que caso $\Delta f_{i+1}>\Delta f_{i}$ ou $\Delta f_{i}>\Delta f_{i+1}, \hat{r}_{i}$ tem um valor próximo de 1 . E se $\Delta f_{i+1}$ e $\Delta f_{i}$ apresentam valores iguais ou bem próximos, $\hat{r}_{i}$ tem um valor próximo de 0 .

Define-se $r_{i}$ tal que

$$
r_{i}=\max \left(\hat{r}_{i}, \hat{r}_{i+1}\right),
$$

permitindo avaliar o indicador de suavidade em uma determinada região.

Os pesos $(\sigma)$ que indicam se determinado ponto é ou não contínuo são dados por

$$
\sigma_{i}=1-r_{i}
$$

sendo necessário considerar um valor limiar $\sigma_{\max }$ tal que $\sigma_{i} \leq \sigma_{\max }$ implica em $\sigma_{i}=0$ e consequentemente em uma região com função descontínua. Já $\sigma_{i}>\sigma_{\max }$ implica em $\sigma_{i}=1$ caracterizando uma região com função suave.

\section{C.3 Kim}

A partir da ideia do indicador de suavidade apresentada por Yee em [33], Kim [14] constrói um novo indicador de suavidade com aplicação em métodos numéricos híbridos. Em regiões consideradas com função suave é utilizado do método central de ordem 6, já para as regiões descontínuas é aplicado o método WENO de ordem 5.

Esse detector tem como principal objetivo identificar quais regiões podem ser consideradas com função suave ou com função descontínua, sendo para isso determinado um peso $\sigma_{i}$ tal que se for igual a 1 indicará suavidade e se for 0 indicará descontinuidade. 
Assim, determina-se um indicador de suavidade da seguinte forma:

$$
\hat{r}_{i}=\frac{\left|2 \Delta f_{i+1} \Delta f_{i}\right|+\varepsilon}{\left(\Delta f_{i+1}\right)^{2}+\left(\Delta f_{i}\right)^{2}+\varepsilon}
$$

sendo que $\Delta f_{i+1}=f_{i+1}-f_{i}$ e $\varepsilon$ é um número real positivo para evitar uma possível divisão por zero.

Nota-se na expressão (C.7) que se $\Delta f_{i}=0$ e $\Delta f_{i+1}=0$ tem-se $\hat{r}_{i}=\varepsilon / \varepsilon=1$, ou seja, caso $f$ seja uma função constante, determina-se que esta é suave na região considerada.

Ao escrever o inverso da expressão (C.7), ou seja, $1 / \hat{r}_{i}$ obtém-se

$$
\frac{1}{\hat{r}_{i}}=\frac{\left|\Delta f_{i+1}\right|^{2}+\left|\Delta f_{i}\right|^{2}}{\left|2 \Delta f_{i+1} \Delta f_{i}\right|}=\frac{1}{2}\left(\frac{\left|\Delta f_{i+1}\right|}{\left|\Delta f_{i}\right|}+\frac{\left|\Delta f_{i}\right|}{\left|\Delta f_{i+1}\right|}\right)
$$

sendo que $\varepsilon$ é desconsiderado na análise do inverso de $\hat{r}_{i}$ pelo fato de representar um valor pequeno.

Caso $\Delta f_{i+1}>\Delta f_{i}$ ou $\Delta f_{i}>\Delta f_{i+1}, 1 / \hat{r}_{i}$ tem um valor muito alto, tal que $\hat{r}_{i}$ será próximo de zero, caracterizando uma possível descontinuidade em torno do ponto. Porém, quando $\Delta f_{i+1} \mathrm{e}$ $\Delta f_{i}$ apresentam valores iguais ou bem próximos, $\hat{r}_{i}$ será aproximadamente 1 , caracterizando uma região com função suave.

Baseado em [23], o valor de $\varepsilon$ é calculado através de

$$
\varepsilon=\frac{0.9 r_{c}}{1-0.9 r_{c}} \xi^{2},
$$

em que $\xi$ e $r_{c}$ atuam como valores limiares. Nota-se que $\xi$ em (C.9) representa um valor pequeno, além dos valores de $r_{c}$ e $\hat{r}_{i}$ pertencerem ao intervalo $[0,1]$.

Define-se $r_{i}$ tal que

$$
r_{i}=\min \left(\hat{r}_{i-1}, \hat{r}_{i}, \hat{r}_{i+1}, \hat{r}_{i+2}\right),
$$

permitindo avaliar o indicador de suavidade em uma determinada região.

Os pesos que indicam se determinado ponto é ou não contínuo são representados por $\sigma_{i}$, com

$$
\sigma_{i}=\min \left(1, \frac{r_{i}}{r_{c}}\right)
$$

Assim, $\sigma_{i}<1$ implica em uma região com função descontínua, ou seja, $\sigma_{i}=0$ e $\sigma_{i}=1$ em uma região com função suave.

Observa-se que a principal função do valor limiar $r_{c}$ é atuar como um delimitador, ou seja, se $\hat{r}_{i}$ possuir um valor menor do que $r_{c}$, a região é considerada como sendo descontínua, como pode ser visto na equação (C.11). 


\section{C.4 Hill}

O principal objetivo desse detector é identificar quais regiões podem ser consideradas com função suave ou descontínua, sendo para isso determinado um peso $\sigma$. A principal ideia apresentada em [11] é utilizar o indicador de suavidade do método WENO [13], tal que

$$
I S_{k}=\sum_{l=1}^{l=r-1} \int_{x_{i-\frac{1}{2}}}^{x_{i+\frac{1}{2}}} d x^{2 l-1}\left(p_{k}^{(l)}(\xi)\right)^{2} d \xi
$$

Para esse caso foi considerado o método WENO de ordem 5, sendo que $r=3$. Assim, os valores de $I S_{0}, I S_{1}$ e $I S_{2}$ são

$$
\begin{aligned}
& I S_{0}=\frac{13}{12}\left(f_{i-2}-2 f_{i-1}+f_{i}\right)^{2}+\frac{1}{4}\left(f_{i-2}-4 f_{i-1}+3 f_{i}\right)^{2}, \\
& I S_{1}=\frac{13}{12}\left(f_{i-1}-2 f_{i}+f_{i+1}\right)^{2}+\frac{1}{4}\left(f_{i-1}-f_{i+1}\right)^{2}, \\
& I S_{2}=\frac{13}{12}\left(f_{i}-2 f_{i+1}+f_{i+2}\right)^{2}+\frac{1}{4}\left(3 f_{i}-4 f_{i+1}+f_{i+2}\right)^{2} .
\end{aligned}
$$

Para realizar a combinação do método numérico híbrido, Hill em [11] utiliza o método central para as regiões com função suave e o WENO [13] para calcular as derivadas espaciais nas regiões com função descontínua. Sendo assim, Hill utiliza dos próprios indicadores de suavidade $\left(I S_{k}\right)$ do método WENO para determinar o detector de descontinuidades.

Os pesos $\sigma$ são determinados da seguinte maneira:

$$
\sigma_{i}=\frac{\max \left(I S_{k}\right)}{\min \left(I S_{k}\right)+\varepsilon}
$$

em que $\varepsilon$ é um número real positivo para evitar uma possível divisão por zero.

Para determinar se a região é com função suave ou descontínua, considera-se um valor limiar $\sigma_{\max }$ tal $\sigma_{i} \geq \sigma_{\max }$ resulta em uma região com função descontínua, ou seja, $\sigma_{i}=0$ e $\sigma_{i}<\sigma_{\max }$ em uma região com função suave.

\section{C.5 Pirozzoli}

Esse detector tem também como principal função identificar regiões que são descontínuas e regiões que podem ser consideradas suaves, sendo para isso determinado um peso $\sigma_{i}$ de forma que se for igual 1, indicará que a região apresenta função suave, e se for 0 indicará descontinuidade.

Assim, é determinado um indicador de suavidade $r_{i}$ tal que

$$
r_{i}=\left|f_{i+1}-f_{i}\right|,
$$


sendo que se $f_{i+1}>>f_{i}$ ou $f_{i}>>f_{i+1}$, o valor de $r_{i}$ será alto, caracterizando a descontinuidade em torno do ponto $i$. Porém, se $f_{i+1}$ e $f_{i}$ forem iguais ou tiverem valores próximos, então $r_{i}$ terá um valor próximo de zero, caracterizando a suavidade em torno do ponto.

Com o indicador de suavidade determinado, define-se o peso $\sigma_{i}$ como

$$
\sigma_{i}=\left\{\begin{array}{ll}
1, & \text { se } r_{i-1} \leq \tilde{r} \text { e } r_{i} \leq \tilde{r} \text { e } r_{i+1} \leq \tilde{r} \\
0, & \text { caso contrário }
\end{array},\right.
$$

em que $\tilde{r}$ é um valor limiar dependente da função em questão.

Nota-se que a desigualdade em (C.16) deve ser satisfeita para que a região possa ser considerada suave. Assim, se qualquer um dos indicadores $r_{i-1}, r_{i}$ ou $r_{i+1}$ foi maior que o valor limiar $\tilde{r}$, a região passa a ser considerada com função descontínua.

\section{C.6 Zhou}

A principal ideia desse detector está em determinar quais regiões podem ser consideradas suaves e quais regiões podem ser consideradas descontínuas. Para isso, será considerado um indicador de suavidade, cuja expressão é dada por

$$
r_{i}=\frac{\left|2 \Delta f_{i+1} \Delta f_{i}\right|+\varepsilon}{\left(\Delta f_{i+1}\right)^{2}+\left(\Delta f_{i}\right)^{2}+\varepsilon},
$$

sendo que $\Delta f_{i}=f_{i}-f_{i-1}$ e $\varepsilon$ é um número real positivo para evitar uma possível divisão por zero. Assim, como $\varepsilon$ representa um valor bem pequeno, este será desconsiderado no cálculo do inverso de $r_{j}$.

Nota-se que ao escrever o inverso da expressão (C.17), ou seja, $\frac{1}{r_{i}}$, tem-se

$$
\frac{1}{r_{i}}=\frac{1}{2}\left(\frac{\left|\Delta f_{i+1}\right|}{\left|\Delta f_{i}\right|}+\frac{\left|\Delta f_{i}\right|}{\left|\Delta f_{i+1}\right|}\right) .
$$

Se $\Delta f_{i+1}>\Delta f_{i}$ ou $\Delta f_{i}>\Delta f_{i+1}, 1 / r_{i}$ tem um valor muito alto, e consequentemente $r_{i}$ será próximo de zero, caracterizando uma possível descontinuidade em torno do ponto. Porém, quando $\Delta f_{i+1}$ e $\Delta f_{i}$ apresentam valores iguais ou bem próximos, $r_{i}$ será próximo de 1 , caracterizando uma região com função suave.

Segundo Ren et al [23], o valor de $\varepsilon$ é escolhido através de

$$
\varepsilon=\frac{0.9 r_{c}}{1-0.9 r_{c}} \xi^{2}
$$

em que $\xi$ e $r_{c}$ atuam como um valor limiar. Nota-se que o valor de $r_{i}$ pertence ao intervalo [0,1]. 
Os pesos que indicarão se determinado ponto é ou não contínuo são representados por $\sigma$, de tal forma que

$$
\sigma_{i}=\min \left(1, \frac{r_{i}}{r_{c}}\right)
$$

Assim, os pontos que forem considerados suaves terão como valor $\sigma_{i}=1 \mathrm{e}$ os considerados descontínuos terão em $\sigma$ um valor diferente de 1 . Para os pontos que receberam o valor de $\sigma_{i}$ como sendo 1 nada mais precisa ser feito, já que de fato são suaves. Porém, os pontos que receberam um valor diferente em $\sigma_{i}$ podem na verdade representar uma vizinhança suave, e devem ser analisados mais detalhadamente.

Então, é proposto um segundo teste para os pontos que apresentam valores para $\sigma_{i}$ menores que 1. Este é responsável por retirar os pontos que foram considerados como descontínuos e que na verdade são suaves.

Se ambas as desigualdades

$$
\alpha_{1}\left(\left|\Delta f_{i}\right|+\beta_{1}\left|\Delta f_{i-1}\right|+\beta_{1}\left|\Delta f_{i-2}\right|\right)>\left|\Delta f_{i+1}\right|
$$

$\mathrm{e}$

$$
\alpha_{2}\left(\left|\Delta f_{i+1}\right|+\beta_{2}\left|\Delta f_{i+2}\right|+\beta_{2}\left|\Delta f_{i+3}\right|\right)>\left|\Delta f_{i}\right|,
$$

forem satisfeitas, então $\sigma_{i}$ é modificado para 1 , caso contrário o valor considerado será 0 . Nota-se que $\alpha_{1}\left(1+2 \beta_{1}\right)>1$ e $\alpha_{2}\left(1+2 \beta_{2}\right)>1$.

A relação $\alpha_{1}\left(1+2 \beta_{1}\right)>1$ pode ser obtida expandindo em série de Taylor cada termo da desigualdade (C.21),

$$
\left(\alpha_{1}+2 \alpha_{1} \beta_{1}\right) f^{\prime}+\left(-\frac{1}{2} \alpha_{1}-4 \alpha_{1} \beta_{1}\right) d x f^{\prime \prime}+O\left(d x^{3}\right)>f^{\prime}+\frac{1}{2} d x f^{\prime \prime}+O\left(d x^{3}\right) .
$$

Assim, pelo primeiro termo obtido tem-se que $\left(\alpha_{1}+2 \alpha_{1} \beta_{1}\right)>1$, que pode ser reescrito com $\alpha_{1}$ em evidência, obtendo $\alpha_{1}\left(1+2 \beta_{1}\right)>1$.

Analogamente para a desigualdade (C.22) obtém-se a relação $\left(\alpha_{2}+2 \alpha_{2} \beta_{2}\right)>1$.

No cálculo de $r_{i}$ apresentado em (C.17) é possível ver que se a razão de $\left|\Delta f_{i+1}\right|$ por $\left|\Delta f_{i}\right|$ é muito maior ou menor que 1 , então $\sigma_{i}<1$. Entretanto, isso não significa que existe uma descontinuidade em torno do ponto $i$, pois para uma função que apresenta grandes oscilações, o detector apresentado em (C.17) indicará a existência de algumas descontinuidades quando na verdade toda a função é suave. Sendo assim, para verificar de fato a descontinuidade, mais informações são obtidas na vizinhança do ponto em questão.

Por simplicidade e sem perda de generalidade, será discutido apenas o caso em que $\left|\Delta f_{i}\right|>$ $\left|\Delta f_{i+1}\right|$. Assim, se a desigualdade (C.21) não for verdadeira, isso implica que a média de $\left|\Delta f_{i-1}\right|$ e $\left|\Delta f_{i-2}\right|$ é menor que $\left|\Delta f_{i+1}\right|$, concluindo que $\left|\Delta f_{i}\right|$ possui o maior valor dentre todos os presentes na desigualdade. Com isso, conclui-se a probabilidade de existir uma descontinuidade em torno de $i$. 
Caso a desigualdade (C.21) é satisfeita, é necessário verificar a desigualdade (C.22). Se esta é satisfeita, nota-se que $\left|\Delta f_{i}\right|$ não possui um valor muito maior que $\left|\Delta f_{i+2}\right|$ e $\left|\Delta f_{i+3}\right|$, concluindose que a função é suave em torno desse ponto. Porém, se a desigualdade (C.22) não é verdadeira, então tem-se a informação que $\left|\Delta f_{i}\right|$ é muito maior que $\left|\Delta f_{i+2}\right|$ e $\left|\Delta f_{i+3}\right|$, sendo possível afirmar que existe uma descontinuidade próxima do ponto $i$.

Após aplicado os dois passos, $\sigma_{i}$ é definido como sendo 0 para a região com função descontínua, e $\sigma_{i}$ é 1 quando a região apresenta função suave. 

[1] Abdalla, A. H.; Zahran, Y. H.; Kaltayev, A. A hybrid WENO scheme for conservation laws. Applied Mathematics Sciences, v. 4, p. 3327-3344, 2010.

[2] Adams, N. A.; Shariff, K. A high-resolution hybrid compact-ENO scheme for shockturbulence interaction problems. Journal of Computational Physics, v. 127, p. 27-51, 1996.

[3] Amaladas, J. R.; Kamath, H. Accuracy assessment of upwind algorithms for steadystate equations. Computer and Fluids, v. 27, p. 941-962, 1998.

[4] Anderson, J. D. Modern compressilble flow. 3 ed. New York, USA: McGraw-Hill, 2003.

[5] Burbeau, A.; SAgaut, P. Simulation of a viscous compressible flow past a circular cylinder with high-order discontinous Galerkin methods. Computer \& Fluids, v. 31, p. 867889, 2002.

[6] Costa, B.; Don, W. S. A high order hybrid central-WENO finite difference scheme for conservation laws. Journal of Computational Physics, v. 204, p. 209-218, 2007.

[7] Gerrard, J. H. The mechanics of the formation region of vortices behind bluff bodies. Journal of Fluid Mechanics, v. 25, p. 401-413, 1966.

[8] Gersten, K. Immersed bodies, heat exchanger design handbook - single phase fluid flow, vol. 2. Hemisphere Publishing Co, 1983.

[9] Harten, A. Adaptative multiresolution schemes for shock computations. Journal of Computational Physics, v. 115, p. 319-338, 1994.

[10] Hedstrom, G. W. Nonreflecting boundary conditions for nonlinear hyperbolic systems. Journal of Computational Physics, v. 30, p. 222-237, 1979. 
[11] Hill, D. J.; PUllin, D. I. Hybrid tuned center-difference-WENO method for large eddy simulations in the presence of strong shocks. Journal of Computational Physics, v. 194, p. 435-450, 2004.

[12] Hu, X. Y.; WANG, Q.; ADAMS, N. A. An adaptive central-upwind weighted essentially non-oscillatory scheme. Journal of Computational Physics, v. 229, p. 8952 - 8965, 2010.

[13] JiAnG, G.-S.; SHU, C.-W. Efficient implementation of weighted ENO schemes. Journal of Computational Physics, v. 126, p. 202-228, 1996.

[14] KIM, D.; KWON, J. H. A high-order accurate hybrid scheme using a central flux scheme and a WENO scheme for compressible flowfield analysis. Journal of Computational Physics, v. 210, p. 554-583, 2005.

[15] Laney, C. B. Computational gasdynamics. Cambridge University Press, 1998.

[16] LELE, S. K. Compact finite difference schemes with spectral-like resolution. Journal of Computational Physics, v. 103, p. 16-42, 1992.

[17] LI, G.; LU, C.; QIU, J. Hybrid well-balanced WENO schems with different indicators for shallow water equations. Journal of Scientific Computing, v. 51, p. 527-559, 2011.

[18] MARKAKIS, C.; BARACK, L. High-order difference and pseudospectral methods for discontinuous problems. ArXiv e-prints, 2014.

Disponível em: http: / /arxiv.org/abs/1406.4865 (Acessado em 13/04/2015)

[19] Oliveira, M.; Lu, P.; LiU, X.; LiU, C. A new shock/discontinuity detector. International Journal of Computer Mathematics, v. 87, p. 3063 - 3078, 2010.

[20] Peyret, R.; Viviand, H. Computation of viscous compressible flows based on the NavierStokes equations. AGARD, 1975.

[21] Pirozzoli, S. Conservative hybrid compact-WENO schemes for shock-turbulence interaction. Journal of Computational Physics, v. 178, p. 81-117, 2002.

[22] Poinsot, T. J. Boundary conditions for direct simulations of compressible viscous flows. Journal of Computational Physics, v. 101, p. 104-129, 1992.

[23] REN, Y.-X.; LIU, M.; ZHANG, H. A characteristic-wise hybrid compact-WENO scheme for solving hyperbolic conservation laws. Journal of Computational Physics, v. 192, p. 365386, 2003.

[24] Roshko, A. On the drag and shedding frequency of two-dimensional bluff bodies. National Advisory for Committee for Aeronautics, 1954. 
[25] Shahbazi, K.; Albin, N.; Bruno, O. P.; Hesthaven, J. S. Multi-domain Fouriercontinuation/WENO hybrid solver for conservation laws. Journal of Computational Physics, v. 230, p. 8779-8796, 2011.

[26] ShU, C. W.; Osher, S. Efficient implementation of essentially non-oscilatory shock capturing schemes. Journal of Computational Physics, v. 77, p. 439-471, 1988.

[27] Sjörgreen, B.; YeE, H. C. Multiresolution wavelet based adaptative numerical dissipation control for high order methods. Journal of Scientific Computing, v. 20, 2004.

[28] Steger, J. L.; WARming, R. F. Flux vector splitting of the inviscid gasdynamic equations with application to the finite-difference methods. Journal of Computational Physics, v. 40, p. $263-293,1981$.

[29] Tannehill, J. C.; Anderson, D. A.; Pletcher, R. H. Computational fluid mechanics and heat transfer. 2 ed. Washington, DC, USA: Taylor \& Francis, 1997.

[30] Thompson, K. W. Time dependent boundary conditions for hyperbolic systems. Journal of Computational Physics, v. 68, p. 1-24, 1987.

[31] Thompson, K. W. Time dependent boundary conditions for hyperbolic systems, II. Journal of Computational Physics, v. 89, p. 439-461, 1990.

[32] Williamson, C. H. K.; Roshko, A. Vortex formation in the wake of an oscillating cylinder. Journal of FLuids and Structures, v. 2, p. 355-381, 1988.

[33] Yee, H. C.; Sandham, N. D.; Djomehri, M. J. Low-dissipative high-order shockcapturing methods using characteristics-based filters. Journal of Computational Physics, v. 150, p. 199-238, 1999.

[34] ZdRaVkovich, M. M. Flow around circular cylinders, vol. 1, [fundamentals]. Oxford University Press, 2007.

[35] ZdRAVKOvich, M. M. Flow around circular cylinders, vol. 2, [applications]. Oxford University Press, 2009.

[36] Zhou, Q.; Yao, Z.; He, F.; Shen, M. Y. A new family of high-order compact upwind difference schemes with good spectral resolution. Journal of Computational Physics, v. 227, p. 1306-1339, 2007. 